Florida International University

FIU Digital Commons

$11-6-2018$

\title{
Investigating the Role of the Proximal Cysteine Hydrogen Bonding Network and Distal Pocket in Chloroperoxidase
}

Elwood Kwong Lam

ekwon001@fiu.edu

Follow this and additional works at: https://digitalcommons.fiu.edu/etd

Part of the Biochemistry Commons

\section{Recommended Citation}

Kwong Lam, Elwood, "Investigating the Role of the Proximal Cysteine Hydrogen Bonding Network and Distal Pocket in Chloroperoxidase" (2018). FIU Electronic Theses and Dissertations. 3898.

https://digitalcommons.fiu.edu/etd/3898

This work is brought to you for free and open access by the University Graduate School at FIU Digital Commons. It has been accepted for inclusion in FIU Electronic Theses and Dissertations by an authorized administrator of FIU Digital Commons. For more information, please contact dcc@fiu.edu. 


\title{
FLORIDA INTERNATIONAL UNIVERSITY
}

Miami, Florida

\section{INVESTIGATING THE ROLE OF THE PROXIMAL CYSTEINE HYDROGEN BONDING NETWORK AND DISTAL POCKET IN CHLOROPEROXIDASE}

\author{
A dissertation submitted in partial fulfillment of \\ the requirements for the degree of \\ DOCTOR OF PHILOSOPHY \\ in \\ BIOCHEMISTRY
}

by

Elwood Kwong Lam

2018 
To: Dean Michael R. Heithaus

College of Arts, Sciences, and Education

This dissertation, written by Elwood Kwong Lam, and entitled Investigating the Role of the Proximal Cysteine Hydrogen Bonding Network and Distal Pocket in Chloroperoxidase, having been approved in respect to style and intellectual content, is referred to you for judgment.

We have read this dissertation and recommend that it be approved.

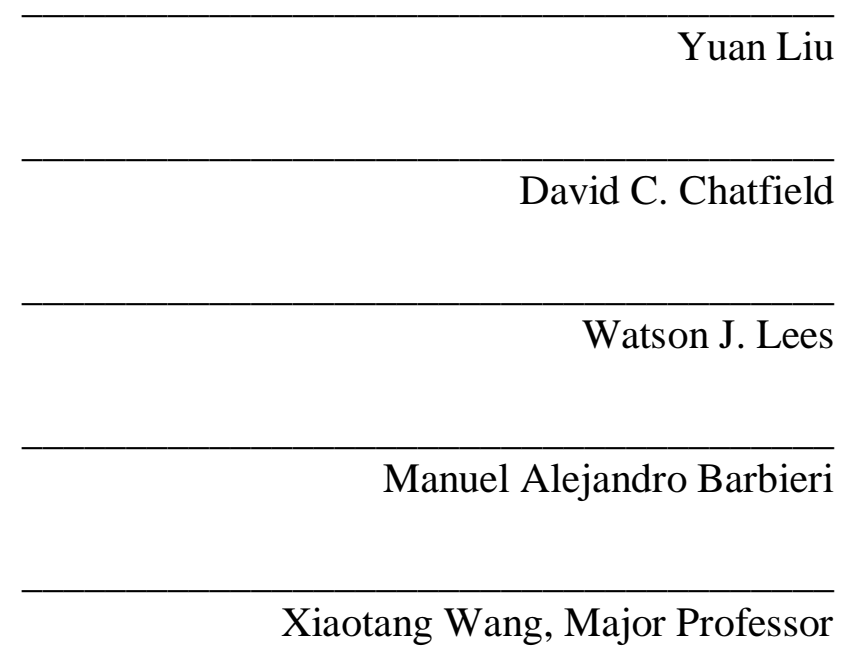

Date of Defense: November 6, 2018

The dissertation of Elwood Kwong Lam is approved.

Dean Michael R. Heithaus

College of Arts, Sciences and Education

Andrés G. Gil

Vice President for Research and Economic Development

and Dean of the University Graduate School

Florida International University, 2018 
(C) Copyright 2018 by Elwood Kwong Lam

All rights reserved. 


\section{DEDICATION}

I dedicate this dissertation to my wife Rong Wei, my daughter Ariel Kwong, my mother SuiHing Lam and my brother Byron Kwong. Without their patience, understanding, support, and most of all love, the completion of this work would not have been possible. 


\section{ACKNOWLEDGMENTS}

Fall of 2013 is the semester that I entered the Biochemistry Ph.D. program and my life changed afterward. During my graduate years in Florida International University, I have met numerous faculty and students and the experiences are priceless. The first person I want to acknowledge must be my major professor, Dr. Xiaotang Wang. He, as a mentor as well as an elder, has provided great support, encouragement, and guidance during my time inside and outside of his research lab. I am extremely lucky to have him as my major professor and it is an honor to work for his lab.

I would also like to thank my other committee members, Dr. David Chatfield, Dr. Watson Lees, Dr. Yuan Liu, and Dr. Manuel Alejandro Barbieri, for their valuable time and helpful advice in helping me to succeed in school and in my personal life. I want to acknowledge my labmates and friends, Dr. Elena Shersher, Dr. Qinghao He, Yongjian Guo, Xiaoqing Tang, and Zifang Deng, for their helpful discussion and pleasant research environment. I want to give special thanks to my good friends, Yongjun Huang and $\mathrm{Na}$ Zhang, for their assistance during my time as graduate students.

Finally, I would like to acknowledge the Department of Chemistry and Biochemistry at FIU for the financial support. Especially, I want to thank Dr. Palmer Graves and Dr. Uma Swamy for their kindness and guidance during my head teaching assistant's experience. 


\title{
ABSTRACT OF THE DISSERTATION
}

INVESTIGATING THE ROLE OF THE PROXIMAL CYSTEINE HYDROGEN

BONDING NETWORK AND DISTAL POCKET IN CHLOROPEROXIDASE

\author{
by
}

Elwood Kwong Lam

Florida International University, 2018

Miami, Florida

\section{Professor Xiaotang Wang, Major Professor}

Chloroperoxidase (CPO) is one of the most versatile heme enzymes isolated from the marine fungus, Caldariomyces fumago. Functionally, CPO can catalyze four types of reactions: peroxidation (peroxidase-like), dismutation (catalase-like), halogenation (halogenase-like), and peroxygenation (P450-like). Structurally, CPO has distal and proximal pockets that can be best described as a hybrid of classical peroxidase and P450s. As a heme-thiolate protein, CPO contains the conserved proximal Pro28-Cys29-Pro30 stretch found in other members of the family. However, the structural and functional roles of these proline residues remain poorly understood.

Site-directed mutagenesis was undertaken to generates three CPO mutants, P28A-, P30A-, P28A/P30A-CPO. The replacement of the rigid proline with a more flexible alanine residue, freed up the back bone amide for the formation of additional amide-sulfur hydrogen bonds, allowing the investigation of the importance of these residues in CPO catalysis. The three $\mathrm{CPO}$ mutants displayed dramatic differences in ligand binding affinity and catalytic activities relative to WT-CPO. Any mutations on the proline resides within the proximal loop eliminated the halogenation and dismutation activities but enhanced the 
epoxidation and peroxidation activities by 4-14 fold. As for the binding affinity for cyanide, the CPO mutants displayed significantly higher dissociation constant relative to WT-CPO. Our results revealed that Pro28 and Pro30 play important roles in maintaining the versatility of CPO.

As a versatile enzyme, $\mathrm{CPO}$ has great application potential in pharmaceutical and chemical industry due to its ability to catalyze the formation of chiral epoxides. Phe103 and Phe186 located on the distal pocket have been proposed to guard the access of substrates to the ferryl oxygen of the heme center. The interactions of these two phenylalanine residues restricted the size of substrates and regulates CPO's enantioselectivity. F186A- and F103A/F186A-CPO were generated and characterized; the rate of peroxidation and epoxidation were significantly enhanced at the expense of halogenation and dismutation activities. Our results demonstrated that Phe186 played a subtler role relative to Phe103 in terms of substrate specificity and product enantioselectivity of CPO. 


\section{TABLE OF CONTENTS}

CHAPTER

PAGE

I.INTRODUCTION

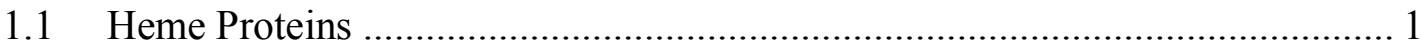

1.2 Heme-containing Oxygenase ................................................................... 3

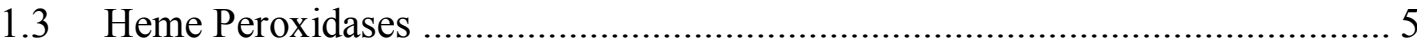

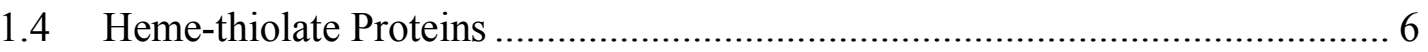

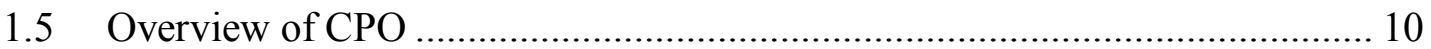

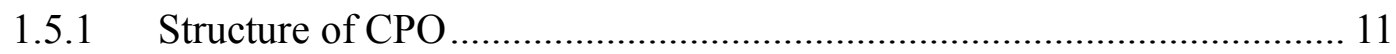

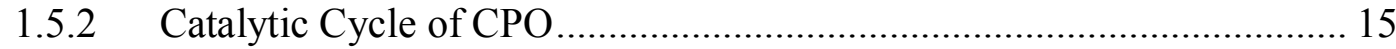

1.5.3 Significant of CPO-catalyzed reactions .................................................. 21

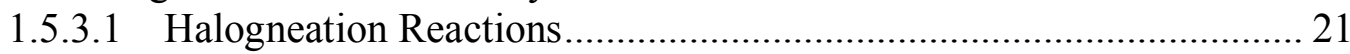

1.5.3.2 Peroxidation Reactions....................................................................... 22

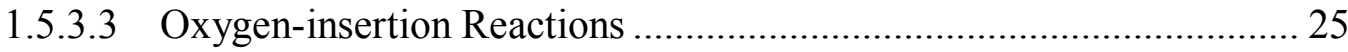

1.5.4 Mutagenesis Approaches on CPO ..................................................... 29

\section{II.INFLUENCE OF THE PRO-CYS-PRO TRIPEPTIDE IN THE PROXIMAL} LOOP ON THE SPECTROSCOPIC PROPERTIES OF CHLOROPEROXIDASE ..32

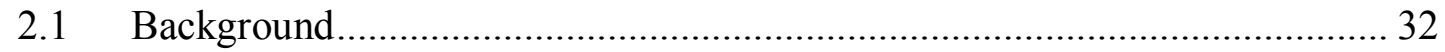

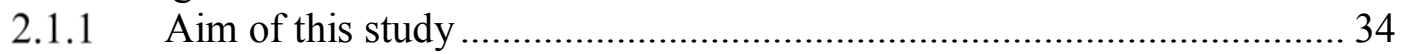

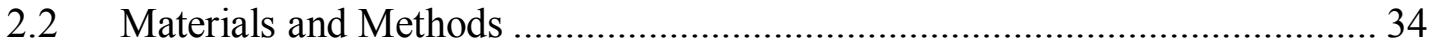

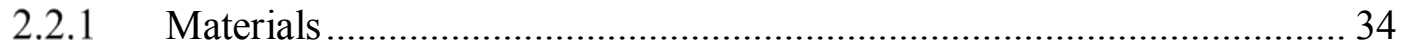

2.2.2 Construction of P28A-, P30A-, and P28A/P30A-CPO genes................. 35

2.2.3 Transformation of mutant plasmid into Aspergillus niger ....................... 38

2.2.4 Expression and Purification of P28A-, P30A-, and P28A/P30A-CPO ... 39

2.2.5 UV-Visible spectroscopic characterization of CPO mutants .................. 40

2.2.6 Ligand Binding Study .................................................................... 40

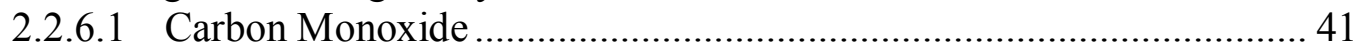

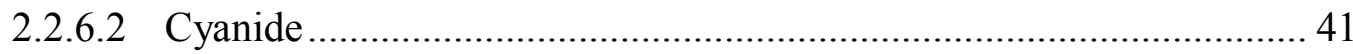

2.2.7 Circular dichroism spectroscopy ....................................................... 42

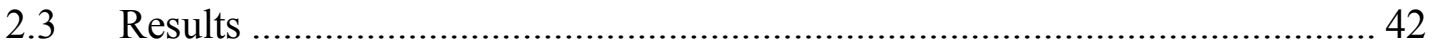

2.3.1 UV-Visible spectroscopic properties of WT-, P28A-, P30A-,

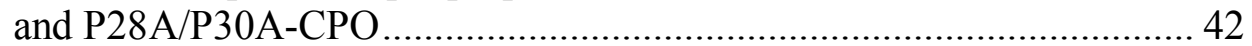

2.3.2 pH study on P28A-, P30A-, and P28A/P30A-CPO ............................... 44

2.3.3 Carbon Monoxide Binding Study on WT-, P28A-, P30A-, and P28A/P30A-CPO ........................................................................... 47

2.3.4 Cyanide Binding Study on WT-, P28A-, P30A-, and

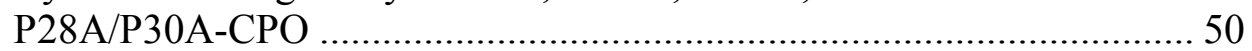

2.3.5 Circular Dichroism Spectroscopy of WT-, P28A-, P30A-, and

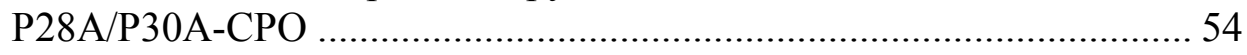

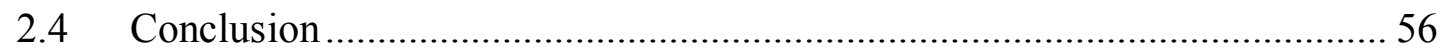




\section{III.INFLUENCE OF THE PRO-CYS-PRO TRIPEPTIDE IN THE PROXIMAL}

LOOP ON THE CATALYTIC PROPERTIES OF CHLOROPEROXIDASE ............59

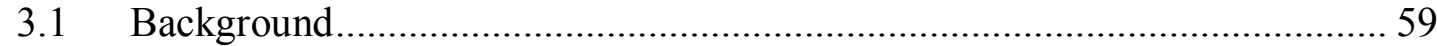

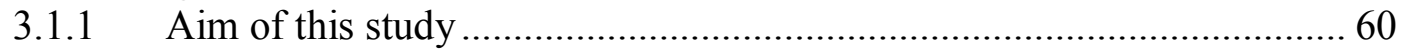

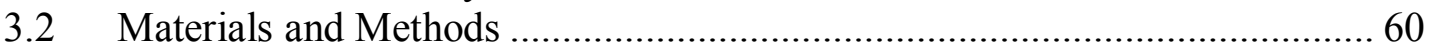

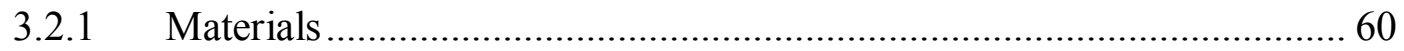

3.2.2 Construction of P28A-, P30A- and P28A/P30A-CPO genes.................. 60

3.2.3 Transformation, Expression and Purification of P28A-, P30A- and P28A/P30A-CPO plasmid in Aspergillus niger ......................................... 60

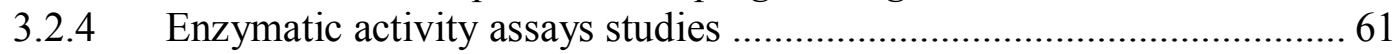

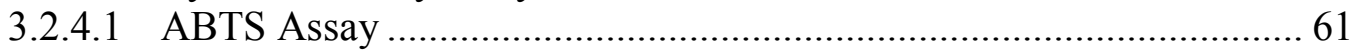

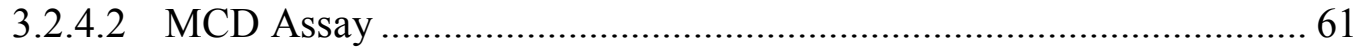

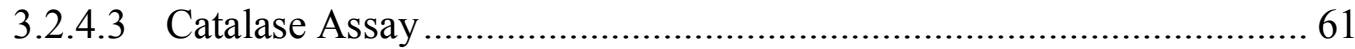

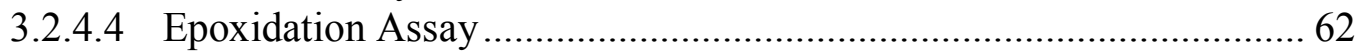

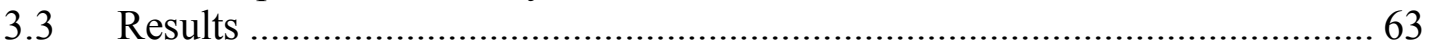

3.3.1 pH Profile of the Catalytic Properties of WT-, P28A-, P30A-, and

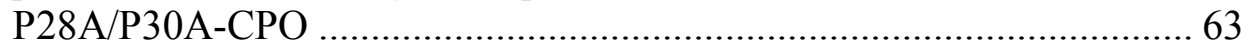

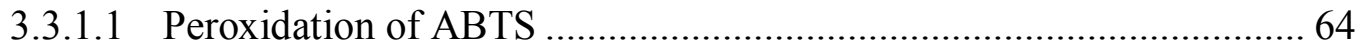

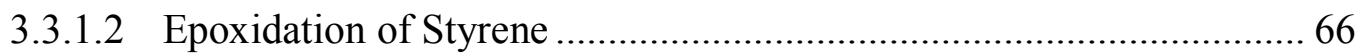

3.3.1.3 Dismutation of Hydrogen Peroxide ..................................................... 68

3.3.1.4 Halogenation of Monochlorodimedone ……………............................ 70

3.3.1.5 Relative Activities ..................................................................... 73

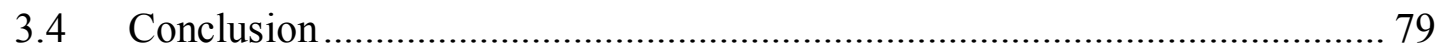

\section{IV.MUTAGENESIS APPROACH ON THE SUBSTRATE-BINDING POCKET}

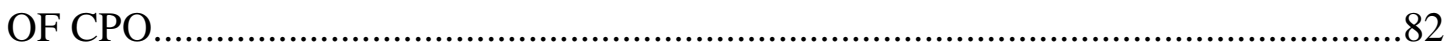

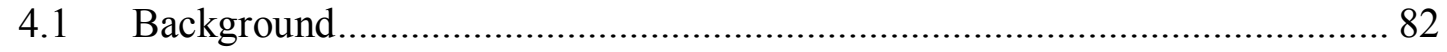

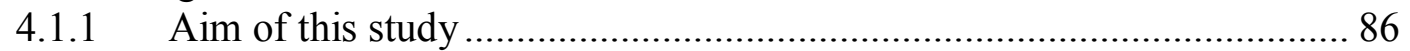

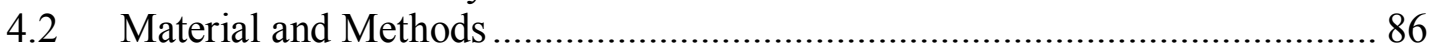

4.2.1 Construction of F186A- and F103A/F186A-CPO genes ........................ 86

4.2.2 Transformation, Expression and Purification of F103A- and F103A/F186A-CPO plasmid in Aspergillus niger .................................. 87

4.2.3 Spectroscopic Characterization ........................................................... 87

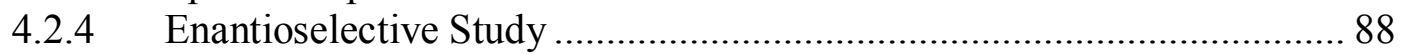

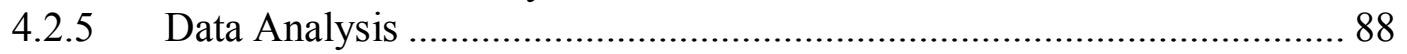

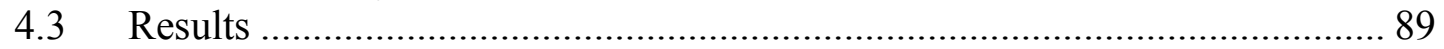

4.3.1 Circular Dichroism Spectroscopy of WT-, F186A-, and ............................

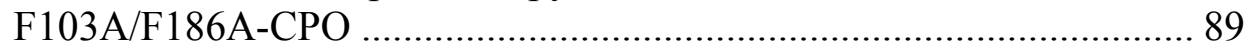

4.3.2 UV-Visible spectroscopic properties of WT-, F186A-, and F103A/F186A-CPO and their carbonmonoxy complex........................... 90

4.3.3 Cyanide Binding Study on WT-, F186A-, and F103A/F186A-CPO ...... 93

4.3.4 Catalytic Properties of WT-, F186A-, and F103A/F186A-CPO............. 94

4.3.4.1 Halogenation Activity of WT-, F186A-, and F103A/F186A-CPO ..... 94

4.3.4.2 Peroxidation Activity of WT-, F186A-, and F103A/F186A-CPO ...... 96 
4.3.4.3 Epoxidation and Dismutation Activity of WT-, F186A-, and

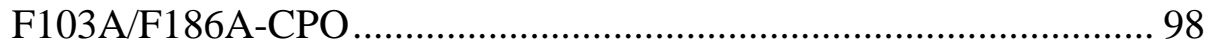

4.3.5 Epoxidation of Styrene and its derivatives catalyzed by WT-,

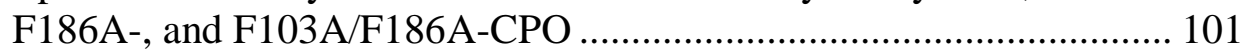

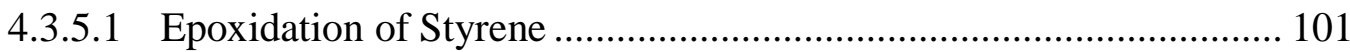

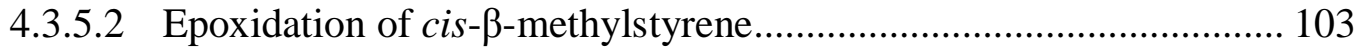

4.3.5.3 Epoxidation of trans- $\beta$-methylstyrene …………………................... 104

4.3.5.4 Epoxidation of trans- $\beta$-ethylstyrene ................................................. 108

4.3.5.5 Epoxidation of trans- $\beta$-propylstyrene …………............................. 110

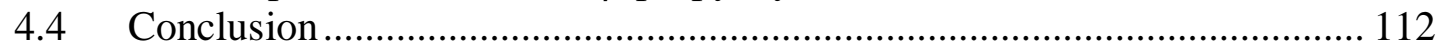

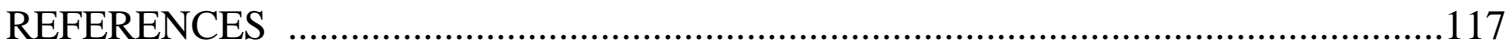

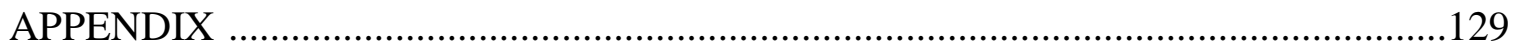

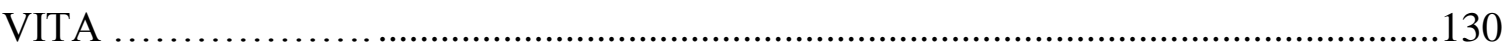




\section{LIST OF TABLES}

TABLE

PAGE

1.1 The effects of the distal environment of CPO on its catalytic activities.....................30

1.2 The effects of the proximal helix of CPO on its catalytic activities. ..........................31

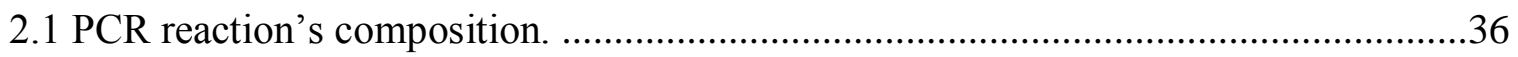

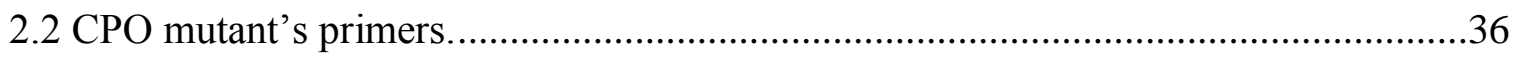

2.3 UV-Vis Spectral properties of WT-CPO and the CPO mutants..............................44

2.4 UV-Vis spectral properties of ferrous and CO-bound WT-CPO and CPO mutants....49

2.5 Dissociation Constant of WT-, P28A-, P30A- and P28A/P30A-CPO in $25 \mathrm{mM}$ phosphate buffer $\mathrm{pH}=5.90$.

2.6 Secondary Structure Composition of WT-, P28A-, P30A- and P28A/P30A-CPO

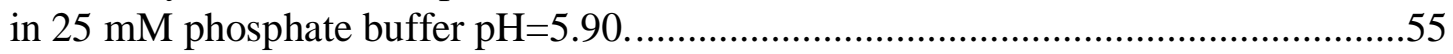

3.1 Extinction coefficient of the Four Assays (ABTS, Epoxidation, Dismutation, and Chlorination).

3.2 Half-reactions with redox potential of hydrogen peroxide and halide ions.

$3.3 \mathrm{pH}$ comparison for the Four Assays (ABTS, MCD, Epoxidation, and Dismutation) 74

4.1 Styrene and its derivatives and their corresponded epoxide products

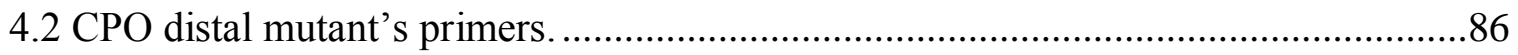

4.3 Secondary Structure Composition of WT-, F186A-, and F103A/F186A-CPO in $25 \mathrm{mM}$ phosphate buffer $\mathrm{pH}=5.90$.

4.4 UV-Vis spectral properties of ferric, ferrous and CO-bound WT-CPO and CPO mutants.

4.5 Enantioselectivity (ee\%) comparison between WT-, F103A-, F186A-, and F103A/F186A-CPO. 


\section{LIST OF FIGURES}

\section{FIGURE}

1.1 The four major types of heme found in heme proteins.

1.2 The catalytic cycle of cytochrome P450 with the redox partner being diflavin cytrochrome $\mathrm{P} 450$ reductase and NADPH

1.3 Four types of reactions catalyzed by heme peroxidase. (1) Peroxidase-catalyzed peroxidation reaction. (2) Peroxidase-catalyzed halogenation reaction. (3) Peroxidase-catalyzed dismutation reaction. (4) Peroxidase-catalyzed peroxygenation reaction

1.4 Structure of (A) Heme-imidazole and (B) Heme-thiolate proteins.

1.5 Structures of P450cam, CPO and iNOS (A) and their respective catalyzed reactions $(\mathrm{B})$

1.6 Alignment of the protein sequences of AaP and CPO with high homology regions highlighted

1.7 Crystal structure of chloroperoxidase from $C$. fumago.

1.8 Slice through surface representation of CPO with the two channels connected to the active site

1.9 The acid-base catalyst of $\mathrm{CPO}(\mathrm{Left})$ and $\mathrm{CcP}(\mathrm{Right})$ are glutamic acid and histidine, respectively

1.10 Proposed Mechanistic Formation of Compound 0, I, II, and III of CPO .16

1.11 Catalytic cycle of CPO as suggested by Dr. Hager. R or RH represented organic substrate while $\mathrm{X}^{-}$represented a halide ion besides fluoride.

1.12 The simplified mechanistic route for CPO-catalyzed halogenation (a), peroxidation (b) and peroxygenation (c) reactions

1.13 The proposed mechanism for chlorination of MCD catalyzed by CPO

1.14 The proposed mechanism for oxygen-insertion reaction via a dual mechanism: (a) an oxoferryl species that inserts oxygen and (b) an iron hydroperoxide species that inserts $\mathrm{OH}^{+}$

1.15 Proposed mechanism for dismutation of hydrogen peroxide catalyzed by CPO......21 
1.16 Halogenation reactions catalyzed by CPO: (A) alkene, (B) alkynes, (C) anisole, (D) phenol, (E) monochlorodimedone, (F) anthracene (aromatic hydrocarbon). .....22

1.17 Initial formation of phenolic radical from the oxidation of fluorophenol catalyzed by CPO and the possible products yielded.

1.18 Two steps one-electron oxidative dehalogenation catalyzed by CPO involving CPO-I and CPO-II .

1.19 CPO-catalyzed sulfoxidation of various sulfides with their corresponded sulfoxides yield and enantiomeric excess percentage .26

1.20 CPO-catalyzed epoxidation of various (A-C) olefins and (D) styrene to their corresponded epoxides yield and enantiomeric excess percentage.....

1.21 CPO-catalyzed progargylic hydroxidation (A), benzylic hydroxylation (B), cyclic conjugated diene hydroxylation $(\mathrm{C})$ and $R-(+)$-limonene hydroxylation in the presence and absence of $\mathrm{KCl}$.

2.1 The hydrogen bonding networks in the proximal pocket of CPO.

2.2 Plasmids used in fungal transformation. (A) pCPO3.I-AmdS plasmid containing A. niger glucoamylase promoter (PglaA), CPO gene from $C$. fumago, A. nidulans anthranilate synthetase terminator (TtrpC), and A. nidulans acetamidase gene (AmdS). (B) pAB4-1 plasmid containing A. niger $\mathrm{PyrG}$ gene and pUC 19 vector.....36

2.3 DNA Electrophoresis of Restriction digestion of pCPO3.I-AmdS 37

2.4 DNA Sequencing of (A) P28A-, (B) P30A-, and (C) P28A/P30A-CPO.....................38

2.5 Eluted fractions collected after gel-filtration chromatography

2.6 UV-Vis Spectra of WT-, P28A-, P30A-, and P28A/P30A-CPO in $50 \mathrm{mM}$ phosphate buffer, $\mathrm{pH} 5.90$.

2.7 UV-Vis Absorption Spectra of P28A-CPO at $\mathrm{pH} 2.50-6.00$. .45

2.8 UV-Vis Absorption Spectra of P30A-CPO at $\mathrm{pH} 2.50-6.00$. .46

2.9 UV-Vis Absorption Spectra of P28A/P30A-CPO at pH 2.50-6.00 .46

2.10 UV-Vis Absorption Spectra of ferric, ferrous and CO-bound WT-CPO. 47

2.11 UV-Vis Absorption Spectra of ferric, ferrous and CO-bound P28A-CPO. .48

2.12 UV-Vis Absorption Spectra of ferric, ferrous and CO-bound P30A-CPO .48 
2.13 UV-Vis Absorption Spectra of ferric, ferrous and CO-bound P28A/P30A-CPO .....49

2.14 Titration of cyanide onto WT-CPO in $25 \mathrm{mM}$ phosphate buffer $\mathrm{pH} 5.90 \ldots \ldots \ldots \ldots . . . .51$

2.15 Titration of cyanide onto $\mathrm{P} 28 \mathrm{~A}-\mathrm{CPO}$ in $25 \mathrm{mM}$ phosphate buffer $\mathrm{pH} 5.90 \ldots \ldots \ldots \ldots . . .52$

2.16 Titration of cyanide onto P30A-CPO in 25mM phosphate buffer $\mathrm{pH} 5.90 \ldots \ldots \ldots \ldots . . .52$

2.17 Titration of cyanide onto P28A/P30A-CPO in 25mM phosphate buffer $\mathrm{pH} 5.90 \ldots . .53$

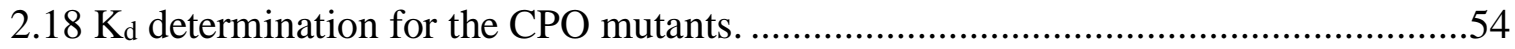

2.19 Secondary CD Spectra of $1.3 \mu \mathrm{M}$ WT and mutant CPO in $25 \mathrm{mM}$ phosphate

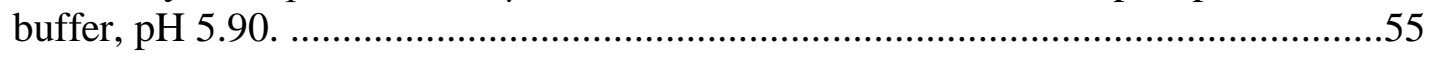

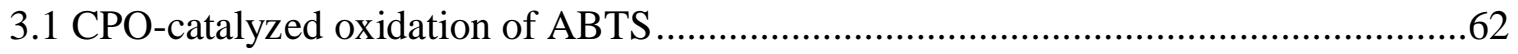

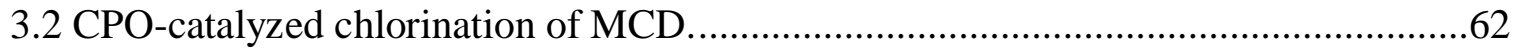

3.3 CPO-catalyzed epoxidation of styrene yielding $R$-styrene oxide and $S$-styrene oxide

3.4 Peroxidation of ABTS catalyzed by WT- and P28A/P30A-CPO monitor at $405 \mathrm{~nm}$.

3.5 Specific activity of WT-, P28A-, P30A- and P28A/P30A-CPO for ABTS assay at $\mathrm{pH}$ 2.50-6.00. (Some error bars are not visible on the graph)

3.6 Epoxidation of Styrene catalyzed by WT- and P30A-CPO monitor at 262nm.

3.7 Specific activity of WT-, P28A-, P30A- and P28A/P30A-CPO for Epoxidation assay at $\mathrm{pH} 3.00-5.50$. (Some error bars are not visible on the graph)

3.8 Dismutation of hydrogen peroxide catalyzed by WT- and P30A-CPO monitor at $240 \mathrm{~nm}$

3.9 Specific activity of WT-, P28A-, P30A- and P28A/P30A-CPO for Epoxidation assay at $\mathrm{pH}$ 2.00-5.50. (Some error bars are not visible on the graph)

3.10 Chlorination of MCD catalyzed by WT- and P30A-CPO monitor at $278 \mathrm{~nm}$. 71

3.11 Specific activity of WT-, P28A-, P30A- and P28A/P30A-CPO for chlorination of MCD assay at $\mathrm{pH}$ 2.00-6.00. (Some error bars are not visible on the graph) .......71 
3.12 Specific activity of WT-, P30A- and P28A/P30A-CPO for bromination of MCD assay at $\mathrm{pH}$ 2.00-6.00. (Some error bars are not visible on the graph)

3.13 Relative activity of WT-, P28A-, P30A- and P28A/P30A-CPO for Epoxidation assay at $\mathrm{pH}=5.00$

3.14 Relative activity of WT-, P28A-, P30A- and P28A/P30A-CPO for dismutation of hydrogen peroxide assay at $\mathrm{pH}=5.00$

3.15 Relative activity of WT-, P28A-, P30A- and P28A/P30A-CPO for dismutation of hydrogen peroxide assay at $\mathrm{pH}=4.00$, optimal $\mathrm{pH}$ for $\mathrm{WT}-\mathrm{CPO}$

3.16 Relative activity of WT-, P28A-, P30A- and P28A/P30A-CPO for chlorination, Left, and bromination, Right, of MCD assay at $\mathrm{pH}=2.75$

3.17 Relative activities of P28A-, P30A- and P28A/P30A-CPO for chlorination, Left, and bromination, Right, of MCD assay at $\mathrm{pH}=2.75$.

3.18 Relative activity of WT-, P28A-, P30A- and P28A/P30A-CPO for peroxidation of ABTS assay at $\mathrm{pH}=2.50$, optimal $\mathrm{pH}$ for WT-CPO.

3.19 Relative activity of WT-, P28A-, P30A- and P28A/P30A-CPO for peroxidation of ABTS assay at $\mathrm{pH}=4.00$

4.1 Substrate-binding pocket on the distal region of CPO

4.2 DNA Sequencing of (A) F186A- and (B) F103A/F186A-CPO

4.3 Successful transformants on 1.2 M Sorbitol acrylamide selective plate

4.4 Secondary CD Spectra of 1.3 $\mu \mathrm{M}$ WT-, F186A-, and F103A/F186A-CPO in $25 \mathrm{mM}$ phosphate buffer, $\mathrm{pH} 5.90$

4.5 UV-Vis spectra of WT-, F186A-, and F103A/F186A-CPO in $25 \mathrm{mM}$ phosphate buffer, pH 5.90 .

4.6 UV-Vis spectra of ferric, ferrous, and CO-bound F186A-CPO in $25 \mathrm{mM}$ phosphate buffer, $\mathrm{pH} 5.90$.

4.7 UV-Vis spectra of ferric, ferrous, and CO-bound F103A/F186A-CPO in $25 \mathrm{mM}$ phosphate buffer, pH 5.90 .

4.8 Titration of cyanide onto F186A-CPO in $25 \mathrm{mM}$ phosphate buffer $\mathrm{pH} 5.90$.

4.9 Titration of cyanide onto F103A/F186A-CPO in $25 \mathrm{mM}$ phosphate buffer pH 5.90 . 
4.10 Specific activity of WT-, F186A- and F103A/F186A-CPO for chlorination of MCD assay at $\mathrm{pH}$ 2.00-6.00. (Some error bars are not visible on the graph).

4.11 Specific activity of WT-, F186A- and F103A/F186A-CPO for bromination of MCD assay at $\mathrm{pH}$ 2.00-6.00. (Some error bars are not visible on the graph).

4.12 Specific activity of WT-, F186A- and F103A/F186A-CPO for peroxidation of ABTS assay at pH 2.50-6.00. (Some error bars are not visible on the graph).

4.13 Relative activity of WT-, F186A- and F103A/F186A-CPO for peroxidation of ABTS assay at pH 2.50 (Left) and 4.00 (Right). (Some error bars are not visible on the graph).

4.14 Specific activity of WT-, F186A- and F103A/F186A-CPO for epoxidation of styrene assay at $\mathrm{pH}$ 3.00-5.50. (Some error bars are not visible on the graph).

4.15 Relative activity of WT-, F186A- and F103A/F186A-CPO for epoxidation of styrene assay at $\mathrm{pH} 4.50$.

4.16 Specific activity of WT-, F186A-, and F103A/F186A-CPO for dismutation of hydrogen peroxide assay at $\mathrm{pH} 2.50-6.00$

4.17 Enantioseparation of styrene oxide catalyzed by WT-CPO on Whelk-O 1 column at room temperature with pure hexane as mobile phase while the absorbance is monitored at $215.7 \mathrm{~nm}$.

4.18 Enantioseparation of styrene oxide catalyzed by F186A-CPO on Whelk-O 1 column at room temperature with pure hexane as mobile phase while the absorbance is monitored at $215.7 \mathrm{~nm}$.

4.19 Enantioseparation of styrene oxide catalyzed by F103A/F186A-CPO on Whelk-O 1 column at room temperature with pure hexane as mobile phase while the absorbance is monitored at $215.7 \mathrm{~nm}$.

4.20 Enantioseparation of $c i s-\beta$-methylstyrene oxide catalyzed by WT-CPO on Whelk-O 1 column at room temperature with pure hexane as mobile phase while the absorbance is monitored at $215.7 \mathrm{~nm}$.

4.21 Enantioseparation of $c i s-\beta$-methylstyrene oxide catalyzed by F186A-CPO on Whelk-O 1 column at room temperature with pure hexane as mobile phase while the absorbance is monitored at $215.7 \mathrm{~nm}$.

4.22 Enantioseparation of $c$ is- $\beta$-methylstyrene oxide catalyzed by F103A/F186A-CPO on Whelk-O 1 column at room temperature with pure hexane as mobile phase while the absorbance is monitored at $215.7 \mathrm{~nm}$. 
4.23 Enantioseparation of trans- $\beta$-methylstyrene oxide catalyzed by WT-CPO on Whelk-O 1 column at room temperature with pure hexane as mobile phase while the absorbance is monitored at $215.7 \mathrm{~nm}$.

4.24 Enantioseparation of trans- $\beta$-methylstyrene oxide catalyzed by F186A-CPO on Whelk-O 1 column at room temperature with pure hexane as mobile phase while the absorbance is monitored at $215.7 \mathrm{~nm}$.

4.25 Enantioseparation of trans- $\beta$-methylstyrene oxide catalyzed by F103A/F186A-CPO on Whelk-O 1 column at room temperature with pure hexane as mobile phase while the absorbance is monitored at $215.7 \mathrm{~nm}$.

4.26 Enantioseparation of trans- $\beta$-ethylstyrene oxide catalyzed by WT-CPO on Whelk-O 1 column at room temperature with pure hexane as mobile phase while the absorbance is monitored at $218.0 \mathrm{~nm}$.

4.27 Enantioseparation of trans- $\beta$-ethylstyrene oxide catalyzed by F186A-CPO on Whelk-O 1 column at room temperature with pure hexane as mobile phase while the absorbance is monitored at $218.0 \mathrm{~nm}$.

4.28 Enantioseparation of trans- $\beta$-ethylstyrene oxide catalyzed by F103A/F186A-CPO on Whelk-O 1 column at room temperature with pure hexane as mobile phase while the absorbance is monitored at $218.0 \mathrm{~nm}$.

4.29 Enantioseparation of trans- $\beta$-propylstyrene oxide catalyzed by WT-CPO on Whelk-O 1 column at room temperature with pure hexane as mobile phase while the absorbance is monitored at $218.0 \mathrm{~nm}$.

4.30 Enantioseparation of trans- $\beta$-propylstyrene oxide catalyzed by F186A-CPO on Whelk-O 1 column at room temperature with pure hexane as mobile phase while the absorbance is monitored at $218.0 \mathrm{~nm}$.

4.31 Enantioseparation of trans- $\beta$-propylstyrene oxide catalyzed by F103A/F186A-CPO on Whelk-O 1 column at room temperature with pure hexane as mobile phase while the absorbance is monitored at $218.0 \mathrm{~nm}$. 


\section{LIST OF ABBREVIATIONS}

ABBREVIATION

aa

AaeUPO/AaP

A. aegerita

ABTS

Ala/A

Amds

A. nidulans

A. niger

APO

Arg/R

A. rusticana

Asn/N

Asp/D

ATCC

CcP

CD

C. fumago

$\mathrm{CN}$

Cpd 0

Cpd I

Cpd II

\section{FULL NAME}

Amino acid

Unspecific peroxygenase from Agrocybe aegerita

Agrocybe aegerita

2,2' -azino-bis(3-ethylbenzothiazoline-6- sulfonic acid)

Alanine

Acetamidase gene

Aspergillus nidulans

Aspergillus niger

Ascorbate peroxidase

Arginine

Armoracia rusticana

Asparagine

Aspartic acid

American type culture collection

Cytochrome $c$ peroxidase

Circular dichroism

Caldariomyces fumago

Cyanide

Compound 0

Compound I

Compound II 


\begin{tabular}{|c|c|}
\hline Cpd III & Compound III \\
\hline Cpd X & Compound X \\
\hline $\mathrm{CPO}$ & Chloroperoxidase \\
\hline CT & Charge transfer \\
\hline Cys/C & Cysteine \\
\hline DCD & Dichlorodimedone \\
\hline DEAE & Diethylaminoethanol \\
\hline DNA & Deoxyribonucleic acid \\
\hline dNTP & Deoxyribonucleotide \\
\hline$e e$ & Enantiomeric excess \\
\hline GLA & Glucoamylase \\
\hline $\mathrm{Gln} / \mathrm{Q}$ & Glutamine \\
\hline Glu/E & Glutamic acid \\
\hline Gly/G & Glycine \\
\hline $\mathrm{His} / \mathrm{H}$ & Histidine \\
\hline HPLC & High performance liquid chromatography \\
\hline HRP & Horseradish peroxidase \\
\hline $\mathrm{KCN}$ & Potassium cyanide \\
\hline $\mathrm{Leu} / \mathrm{L}$ & Leucine \\
\hline LiP & Lignin peroxidase \\
\hline MCD & Monochlorodimedone \\
\hline MM & Minimal medium \\
\hline MPO & Myeloperoxidase \\
\hline
\end{tabular}




$\begin{array}{ll}\text { NAD }(\mathrm{P}) \mathrm{H} & \text { Nicotinamide adenine dinucleotide (phosphate) } \\ \text { NMR } & \text { Nuclear magnetic resonance } \\ \text { NOS } & \text { Nitric oxide synthase } \\ \text { P450 } & \text { Cytochrome P450 monooxygenase } \\ \text { P450cam } & \text { P450 camphor } \\ \text { PCR } & \text { Polymerase chain reaction } \\ \text { PDB } & \text { Protein data base } \\ \text { PglaA } & \text { glucoamylase promoter } \\ \text { Phe/F } & \text { Phenylalanine } \\ \text { Pro/P } & \text { Proline } \\ \text { RR } & \text { Resonance Raman } \\ \text { RZ value } & \text { Reinheitzahl value } \\ \text { SDS-PAGE } & \text { Sodium dodecyl sulfate polyacrylamide gel electrophoresis } \\ \text { Trp/W } & \text { Tryptophan } \\ \text { trpC } & \text { anthranilate synthase terminator } \\ \text { Tyr/T } & \text { Tyrosine } \\ \text { UPO } & \text { Unspecific peroxygenase } \\ \text { UV-Vis } & \text { Ultraviolet-Visible } \\ \text { Val/V } & \text { Valine } \\ \text { WT } & \end{array}$




\section{CHAPTER I \\ INTRODUCTION}

\subsection{Heme Proteins}

Heme or iron-protoporphyrin IX, consists of ferrous iron coordinated to four nitrogens of pyrrolic groups and these groups are joined by methane bridges [1,2]. Besides being coordinated to the four nitrogens of the porphyrin, the heme iron is usually ligated to one or two axial ligands such as histidine, cysteine, tyrosine, methionine, and/or lysine residues within the protein or small molecules such as oxygen, nitric oxide, carbon monoxide, or water [1]. There are four main types of heme: heme a, heme b, heme $\mathrm{c}$ and heme $d_{l}$, depending on the attached side chains along the heme plane as seen in Figure 1.1. The general functions of heme proteins can be simplified into three categories: (1) electron transport, (2) oxygen transport and (3) catalysis of redox reactions [3]. For example, hemoglobin is a heme $b$ type protein and is responsible for the transporting oxygen from the lung to tissues. Among the different four major types of heme, heme b or iron protoporphyrin IX is the most abundant in heme proteins. Type $\mathrm{b}$ heme proteins can utilize two oxidants, peroxides or molecular oxygen, to carry out their functions, separating them into heme peroxidases and heme oxygenases. Heme oxygenase utilizes dioxygen for oxidation/oxygenation of substrates and the most iconic example of this type of heme enzyme is the cytochrome P450's family. Interestingly, the process of oxidation by dioxygen is thermodynamically favored but there is a large kinetic barrier that prevents the progress of these reactions [4]. Therefore, the ability of heme oxygenases to harness the oxidative power of dioxygen has been a great interest to the field of metalloprotein catalysis. As for heme peroxidase, these enzymes catalyze oxidation/peroxidation reactions 
using peroxide instead of dioxygen. The following sections will describe the two types of heme proteins, heme-containing oxygenase and heme peroxidases.

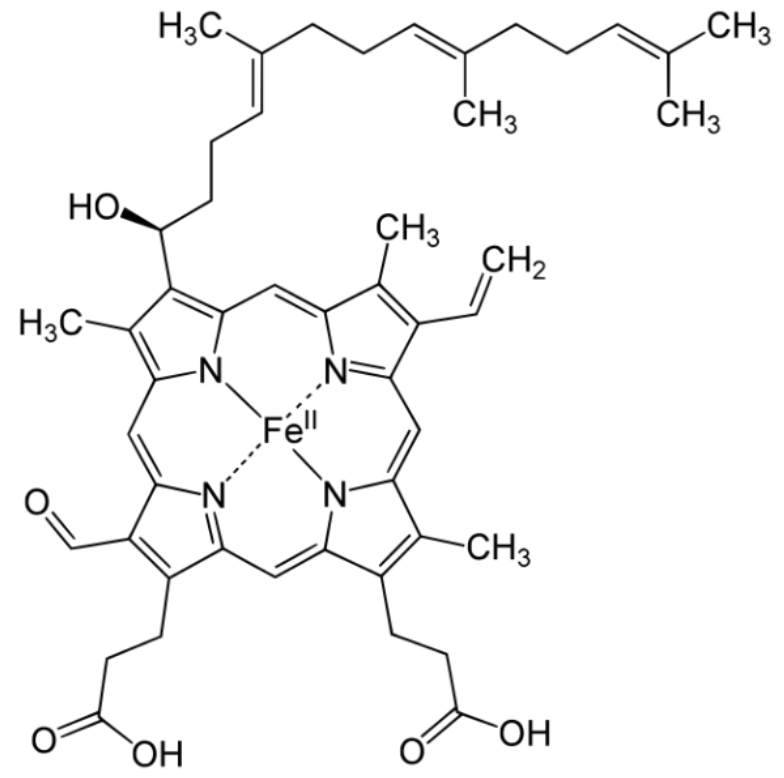

Heme a

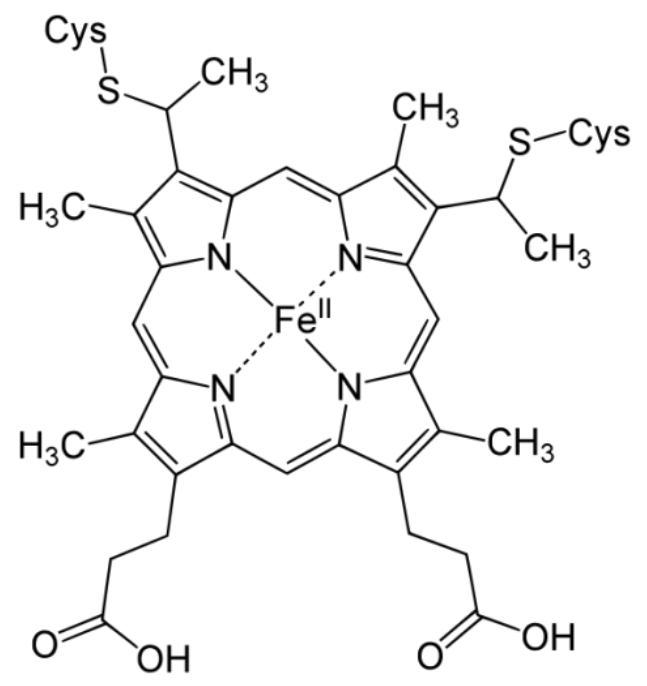

Heme c

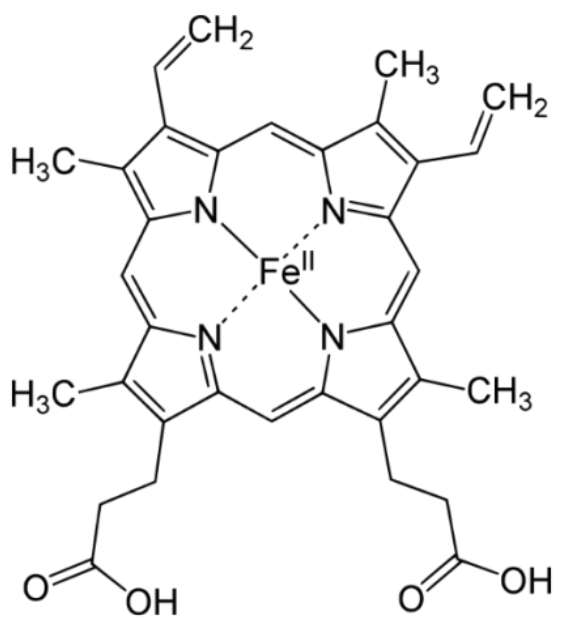

Heme b

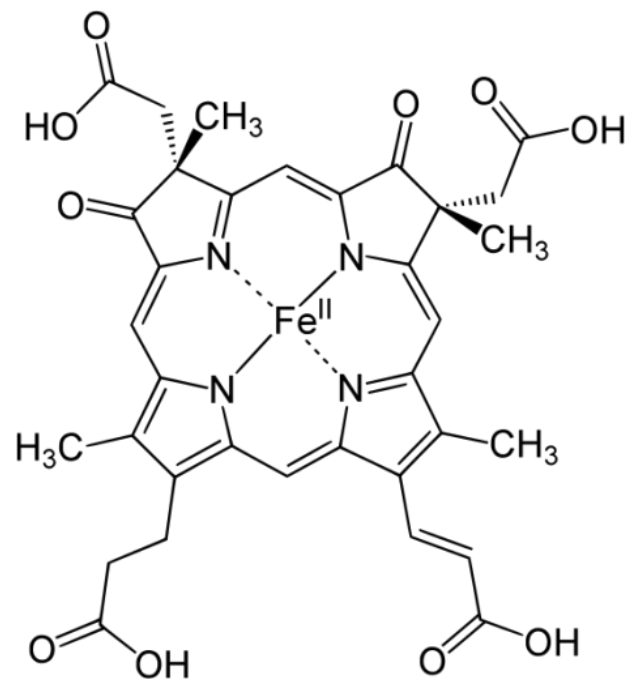

Heme $d_{1}$

Figure 1.1 The four major types of heme found in heme proteins. 


\subsection{Heme-containing Oxygenase}

Heme-containing oxygenase is defined by its ability to activate molecular oxygen and catalyze oxygenation of potential substrates. The most studied heme oxygenase or monooxygenase has to be the cytochrome P450 family as P450 enzymes exist throughout all domains of life catalyzing important biological oxidations such as steroid and lipid biosynthesis, xenobiotic detoxification, and drug metabolism [5-7]. Among these oxidative reactions, P450-catalyzed hydroxylation of inert carbon-hydrogen bond has been a great interest because this type of reaction can only be carried out by the P450-like enzymes which are heme-thiolate proteins, as they share a cysteine residue as proximal ligand [812]. More details of heme-thiolate protein will be discussed in section 1.4.

Another example of heme-containing oxygenase is secondary amine monooxygenase which functions in catalyzing the oxidative dealkylation of secondary amines to aldehydes and primary amines. Structurally, secondary amine monooxygenase contains the heme cofactor that is responsible for catalysis and also flavin mononucleotide and $\mathrm{Fe}_{2} \mathrm{~S}_{2}$ prosthetic groups that have been proposed to play a role in modulating the transport of electrons from NADPH to the heme center [13]. Interestingly, the second purified monooxygenase, secondary amine monooxygenase contains a histidine residue as the proximal ligand unlike P450 enzymes [14]. Therefore, this leads to the question of the structural requirement for activating molecular oxygen as this monooxygenase contains an active site that resembles that of myoglobin.

Besides P450 enzymes and secondary amine monooxygenase, heme oxygenase (HO) is a unique type of heme protein catalyzes the oxidation of heme b leading to the release of carbon monoxide, $\mathrm{Fe}^{3+}$, and biliverdin. The uniqueness of this enzyme is that it does not 
actually contain heme as cofactor; rather, the free heme binds to the enzyme during catalysis forming this transient heme protein state $[4,13]$. Like P450 enzymes and secondary amine monooxygenase, the ferric heme iron of heme oxygenase needs to be reduced to ferrous iron before the binding of molecular oxygen to the heme iron as demonstrated in P450's catalytic cycle in Figure 1.2 [15]. After molecular oxygen is bound to the heme iron, a second electron and two hydrogen protons are needed for the release of a water molecule. The electron transferring process from NADPH to the heme iron is regulated by the enzyme, diflavin cytochrome $\mathrm{P} 450$ reductase, which is used by the three heme oxygenases described $[13,16]$. The axial heme ligand $\mathrm{HO}$ is a histidine residue, in analogy to secondary amine monooxygenase.

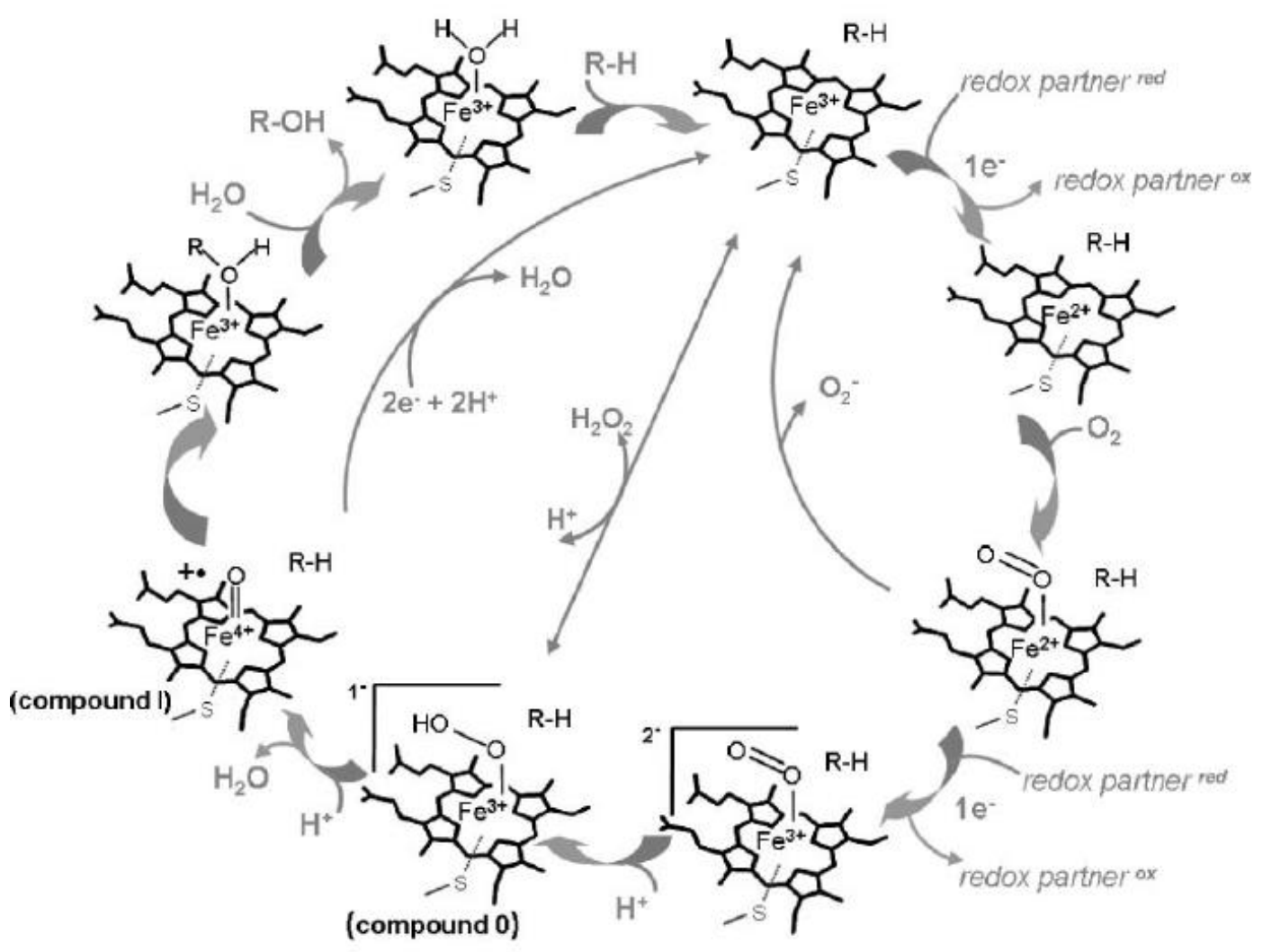

Figure 1.2 The catalytic cycle of cytochrome $\mathrm{P450}$ with the redox partner being diflavin cytrochrome P450 reductase and NADPH [17]. 


\subsection{Heme Peroxidases}

As the heme center of $\mathrm{HO}$ and secondary amine monooxygenase is ligated to a histidine residue, most heme peroxidases are also heme-imidazole ligated proteins. Heme peroxidases use hydrogen peroxide instead of molecular oxygen as oxidizing agent to catalyzes the one- and two-electron oxidation reactions. These reactions include the formation of a radical from the oxidized one-electron donor molecule and two-electron oxidation of halides ions to hypohalous acids (Figure 1.3, reaction $1 \& 2$ ). Examples of classical heme peroxidases include horseradish peroxidase [18], cytochrome c peroxidase [19] and lignin peroxidase [20]. There are four heme peroxidase superfamilies: peroxidasecatalase, peroxidase-cyclooxygenase, peroxidase-chlorite dismutase and peroxidaseperoxygenase superfamily [21, 22]. Each superfamily is distinguished by its distinctive active site and enzymatic activities in addition to peroxidase activity. For example, peroxidase-catalase can catalyze the decomposition of hydrogen peroxide to protect the proteins from oxidative damage (Figure 1.3, reaction 3) [23]. Cytochrome $c$ peroxidase is a classic example of peroxidase-catalase superfamily and it was one of the first heme peroxidase to be crystallized [24]. Thus, $\mathrm{CcP}$ has served as benchmark for the studies of other peroxidases. The structure of $\mathrm{CcP}$ along with others heme peroxidases revealed catalytically important conserved regions found in heme peroxidase-catalase superfamily. For example, the heme of $\mathrm{CcP}$ is coordinated to a histidine (His175) ligand which forms hydrogen bonds with nearby residues such as Asp and Trp or Phe. This proximal HisTrp/Phe-Asp triad along with the distal His-Trp/Phe-Arg triad are conserved in most peroxidases and have been proposed to be catalytic significant [21, 25]. Exceptions include monofunctional catalase, in which the proximal ligand is a tyrosine residue, and 
peroxidase-peroxygenase, a superfamily responsible for catalyzing the oxygen-insertion reaction of organic molecules (Figure 1.3, reaction 4), such as chloroperoxidase and unspecific peroxygenase that contain a cysteine proximal ligand [8]. The different proximal ligand between heme-imidazole and heme-thiolate protein is illustrated by Figure 1.4.

(1) $2 \mathrm{RH}+\mathrm{H}_{2} \mathrm{O}_{2} \rightarrow 2 \mathrm{R}^{\cdot}+2 \mathrm{H}_{2} \mathrm{O} \rightarrow \mathrm{R}-\mathrm{R}+2 \mathrm{H}_{2} \mathrm{O}$

(2) $\mathrm{X}^{-}+\mathrm{H}_{2} \mathrm{O}_{2}+\mathrm{H}^{+} \rightarrow \mathrm{HOX}+\mathrm{H}_{2} \mathrm{O}$

(3) $2 \mathrm{H}_{2} \mathrm{O}_{2} \rightarrow \mathrm{O}_{2}+2 \mathrm{H}_{2} \mathrm{O}$

(4) $\mathrm{RH}+\mathrm{H}_{2} \mathrm{O}_{2} \rightarrow \mathrm{ROH}+\mathrm{H}_{2} \mathrm{O}$

Figure 1.3 Four types of reactions catalyzed by heme peroxidase. (1) Peroxidasecatalyzed peroxidation reaction. (2) Peroxidase-catalyzed halogenation reaction. (3) Peroxidase-catalyzed dismutation reaction. (4) Peroxidase-catalyzed peroxygenation reaction. $\mathrm{RH}$ represents substrates; $\mathrm{X}$ - is the halide ions (Cl-, Br-, or I-)

(A)

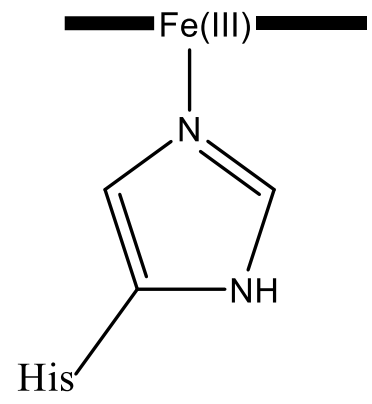

(B)

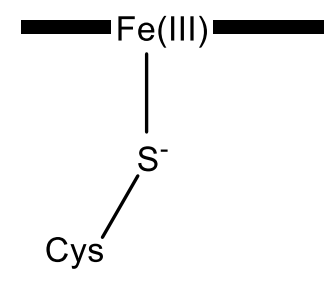

Figure 1.4 Structure of (A) Heme-imidazole and (B) Heme-thiolate proteins.

\subsection{Heme-thiolate Proteins}

Heme-thiolate proteins are hemoproteins with a cysteine as the proximal heme ligand instead of a histidine as found in classical peroxidases. Upon binding of carbon monoxide, the UV-Vis spectrum of heme-thiolate proteins exhibits a prominent Soret peak near 450 $\mathrm{nm}$ for this carbonmonoxy complex while heme-imidazole proteins have a Soret peak around $420 \mathrm{~nm}$ [26-28]. Therefore, carbon monoxide has been used to probe the identity 
of the proximal heme ligand. The absorption peak near $450 \mathrm{~nm}$ is the origin of the name P450 because cytochrome P450 is the first class of hemoprotein to display this spectroscopic characteristic when bound to carbon monoxide. P450 can catalyze a wide range of biological oxidative reactions and the catalytic capability of this protein has been proposed to be modulated by the axial ligand $[8,29]$. The $\mathrm{C} 357 \mathrm{H}$ mutation eliminated the hydroxylating activity of P450cam for camphor and reduced the rate of the reactive species formation $[10,29]$. The dramatic changes in terms of electron donating properties from cysteine to histidine altered the proximal heme environment thus affected the catalytic activity, as observed for C357H-P450cam. Even though heme-thiolate proteins share the same axial cysteine ligand, their primary catalytic functions vary dramatically. For example, P450 catalyzes monooxygenase reactions, CPO catalyzes haloperoxidase reactions, while NOS catalyzes the production of NO through the oxidation of L-arginine (Figure 1.5) [6, 16, 30-32].

$\mathrm{CPO}$ is the first haloperoxidase that was categorized into the heme-thiolate protein family and has been known as one of the most versatile hemoprotein. The primary function of $\mathrm{CPO}$ has been proposed to catalyze the chlorination reaction during the biosynthesis of the antibiotic caldariomycin [33-36]. The spectroscopic signature of the carbonmonoxy complex of ferrous $\mathrm{CPO}$ resembles that of P450 which suggested identity of the proximal ligand between these two enzymes is the same [37]. In addition, the crystal structure of $\mathrm{CPO}$ confirmed the identity of the proximal ligand is a thiolate anion from a cysteine residue [38]. This enzyme has served as a model for studies of heme-thiolate proteins, especially when determining the reactive intermediates in the catalytic cycle because Compound I of P450 is highly unstable, and until recently, capturing this species has 
proven to be problematic. Therefore, Compound I of CPO as well as model-based studies have been used to investigative the reactive species and the mechanism of P450s [39-43]. More recently, the availability of Compound I of P450 has shown that the reactive species of the two heme-thiolate enzymes displayed similar UV/Visible and Mössbauer spectra but differ in electron paramagnetic resonance spectrum [44].

(A)

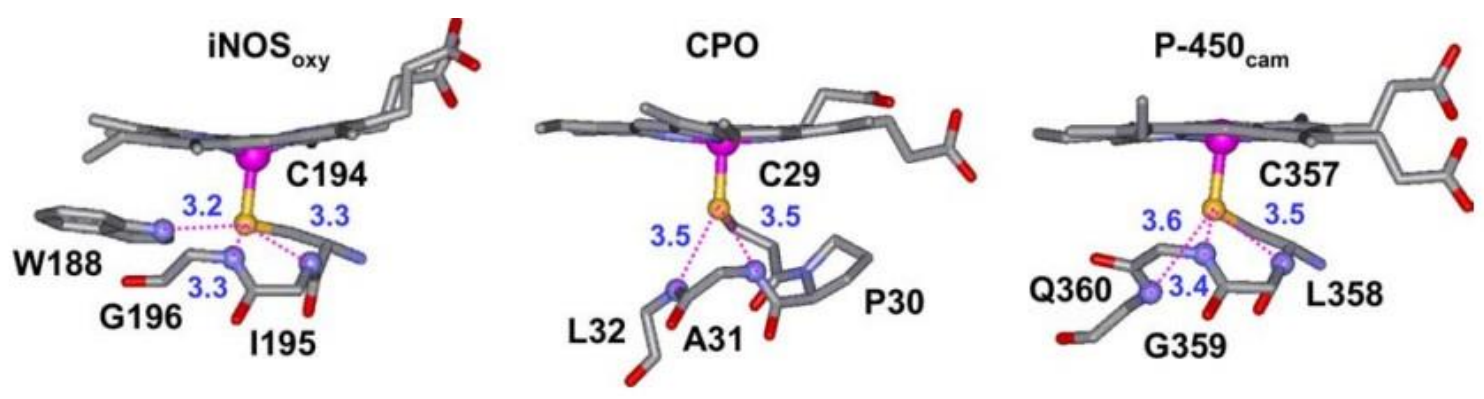

(B)

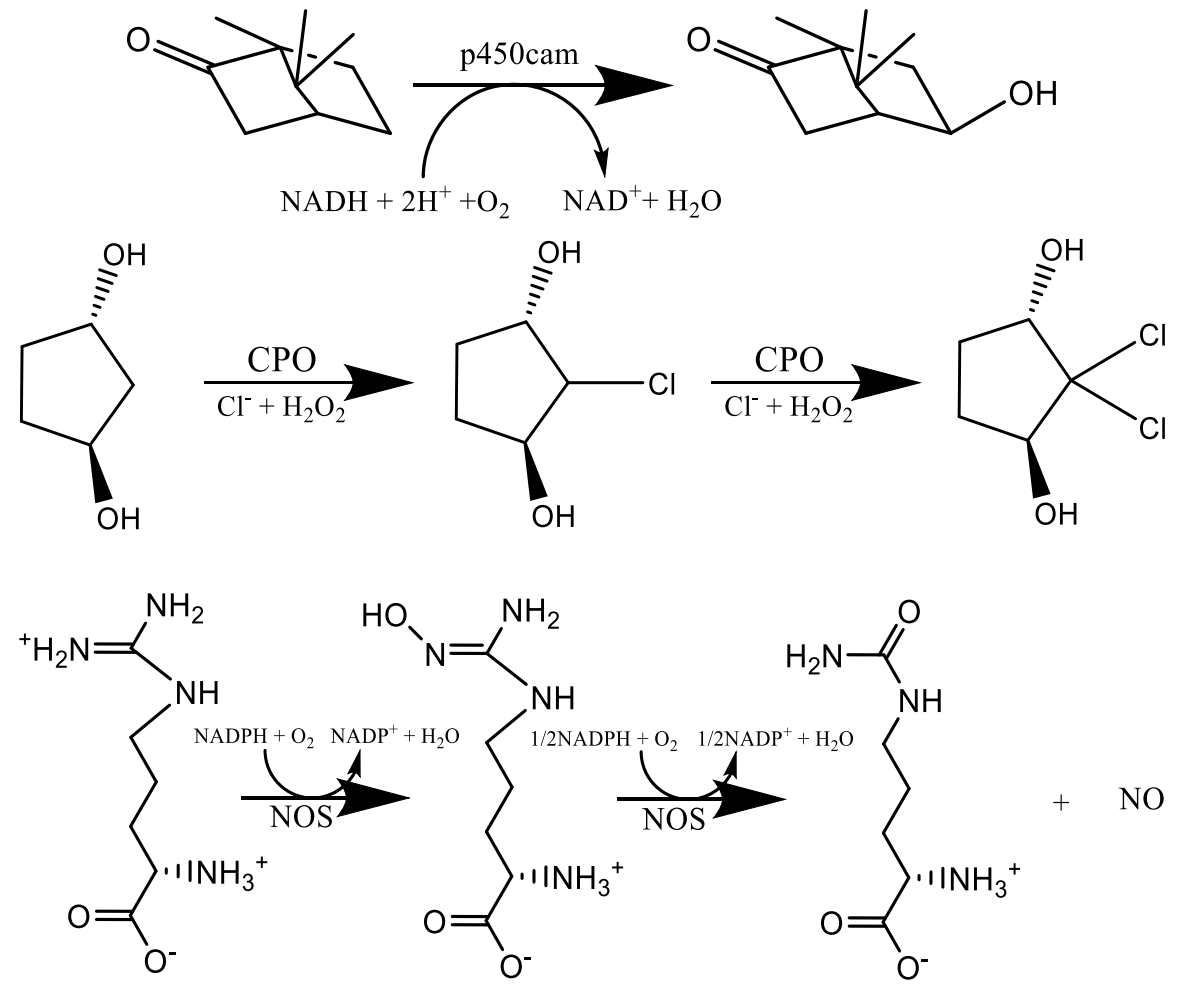

Figure 1.5 Structures of P450cam, CPO and iNOS (A) [32] and their respective catalyzed reactions $(B)$. 
In recent years, the arise of another haloperoxidase that belong in the heme-thiolate protein family is unspecific peroxygenase (UPO) $[45,46]$. This type of enzyme was first discovered in the basidiomycetous fungus, Agrocybe aegerita, known as AaP or AaeUPO. AaP was known as an "alkaline lignin peroxidase" because this enzyme oxidizes veratryl alcohol into its corresponding aldehyde at neutral $\mathrm{pH}[47,48]$. However, the mechanism of the degradation of lignin catalyzed by AaP was found to be different than that of lignin peroxidase as LiP involves the formation of an aryl cation radical while AaP forms a geminal alcohol $[49,50]$. Besides peroxidase activity, AaP can catalyze the halogenation of monochlorodimedone and phenol in $\mathrm{H}_{2} \mathrm{O}_{2}$-dependent manner that resembles CPO [31, 46]. In terms of protein sequence, there is only about $30 \%$ similarity in the N-terminal region, which comprises the proximal region as well as part of the distal pocket between $\mathrm{AaP}$ and $\mathrm{CPO}$ as observed in Figure 1.6 [51]. AaP shares similarity with CPO but AaP displays higher peroxygenase activity toward aliphatic and aromatic hydrocarbons as well as numerous organic substrates [52]. This oxygen-insertion potential of AaP resembles that of P450s. In addition, the spectroscopic characteristics such as the resting ferric state of $\mathrm{AaP}$ are similar to those of $\mathrm{P} 450$, which is in a low-spin state, while ferric CPO exists as a penta-coordinated high-spin species. Therefore, AaP or UPO have been proposed to be the missing link between CPO and P450s.

The rising interest in AaP's peroxygenase activity altered the name of this enzyme from haloperoxidase to aromatic peroxygenase and then to UPO as a result of its unusual/versatile enzymatic activities [53]. The second UPO discovered is CraUPO secreted by Coprinellus radians and it shares much similarity with AaeUPO such as catalyzing the hydroxylation of naphthalene $[45,50]$. As heme-thiolate proteins, UPOs 
catalyze oxygen-insertion into aromatic substrates with great efficiency that are not P450 enzymes, which revealed an alternate potential biocatalyst with several advantages such as a simpler system without the need of flavin reductases [54].

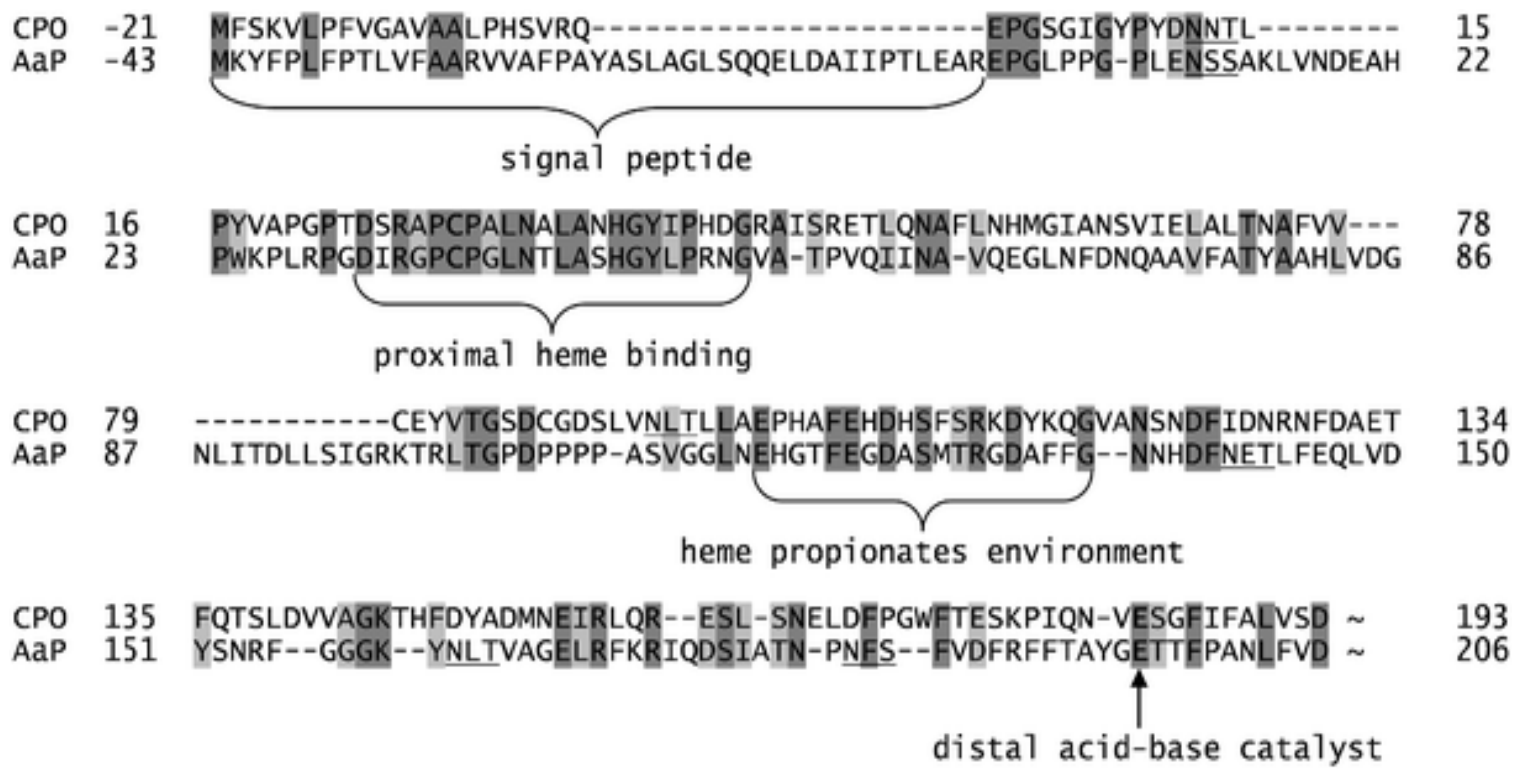

Figure 1.6 Alignment of the protein sequences of AaP and CPO with high homology regions highlighted. Dark gray represented identical residues while light gray is residues with chemical similarity. Underlined residues are the $N$-glycosylation sites [51].

\subsection{Overview of CPO}

Chloroperoxidase (CPO) is isolated from the marine fungus, Cardarimycin fumago by Dr. Hager and his group in early 1960s [33, 34]. This versatile enzyme's primary function as a halogenase catalyzing oxidation of halides such as chloride, bromide and iodide [55, 56]. The halogenating activity of $\mathrm{CPO}$ have been suggested to be the possible natural production of organochlorine compounds in the environment [57]. Besides being haloperoxidase, $\mathrm{CPO}$ can also catalyze other two-election oxidation reactions such as dismutation of hydrogen peroxide as well as peroxygenation reactions. Peroxygenation reactions such as epoxidation of olefins have shown to produce high yield with high 
stereoselectivity. In addition, as a heme peroxidase, $\mathrm{CPO}$ also function as a peroxidase that catalyzes one-electron peroxidation reactions [58]. CPO's versatility was linked to the "peroxidase-P450" hybrid structure of this enzyme [38]. Unraveling the structure-function relationship of CPO have been a great interest since this heme-thiolate protein have great potential for industrial application.

\subsubsection{Structure of CPO}

$\mathrm{CPO}$ is a heavily glycosylated extracellular enzyme which $25-30 \%$ of the molecular weight is carbohydrate [59]. CPO's gene encoded 372 amino acid residues and after the proteolytic cleavage process; 21 aa from the $\mathrm{N}$-terminal signal peptide and 52aa from the C-terminal propeptide were removed leaving the mature protein with 299aa residues and a molecular weight of approximately 42,000 $\mathrm{Da}$ [60]. The crystal structure of CPO revealed that the heme center was mainly surrounded by eight alpha helixes (A-H), three short helices and a short antiparallel beta pair (Figure 1.7) [38]. The structure of CPO is unique among peroxidases and heme-thiolate proteins, but it shares the similarity of a polar substrate-binding pocket and axial ligand, respectively. The proximal cysteine ligand is located in the N-terminal helix A. This helix is orientated perpendicular to the heme plane and possess an electropositive environment that fine-tunes the electronegativity of the axial ligand [38]. In other peroxidases and P450s, the helix containing the axial ligand is usually orientated parallel to the heme $[24,61]$. 


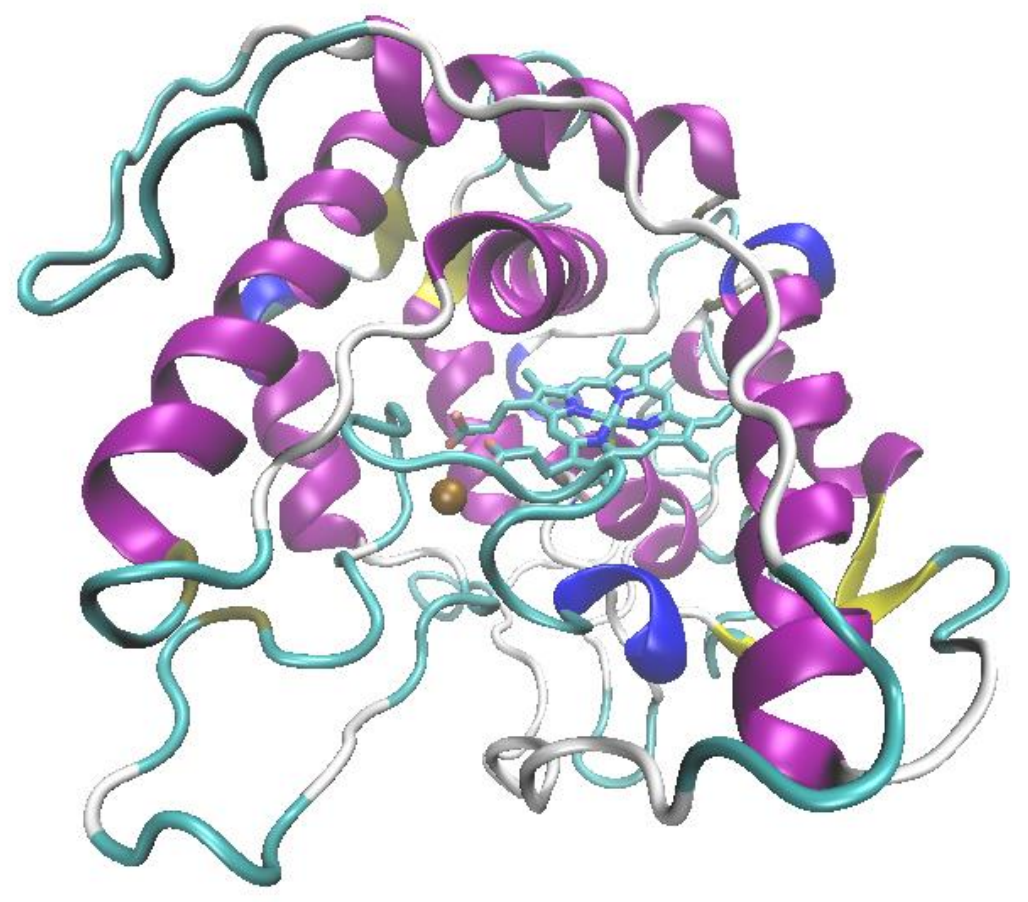

Figure 1.7 Crystal structure of chloroperoxidase from C. fumago [38]. The manganese is represented by the brown sphere near the heme center. PDB file of 1CPO was used to generate this figure.

The orientation of the proximal helix is different between P450 and CPO, but they share a similar three residue stretch around the cysteine ligand, X-Cys-X. For CPO, the stretch is a Pro-Cys-Pro tripeptide which is superimposed to various structures of heme-thiolate proteins. For example, in the proximal helix of $\mathrm{CPO}$ and $\mathrm{AaP}$, they shared a similar structural motif of Arg-Gly/Ala-Pro-Cys-Pro-Ala/Gly-Leu-Asn. This motif is found to be conserved throughout the peroxidase-peroxygenase superfamily (Figure 1.6). Interestingly, the crystal structure of beef liver catalase shows that the axial ligand, Tyr357, is adjacent to the proline residue, Pro358 which been proposed to restrict the movement of the tyrosine [62]. 
The rich electron-donating properties of the proximal thiolate of CPO is modulated by the proximal helix environment. In heme-thiolate proteins, there is an amide-sulfur hydrogen bonding network located in the proximal pocket that regulates the electronegativity of the proximal ligand [8, 11, 63-69]. In CPO, the sulfur atom of Cys29 form an amide-sulfur hydrogen bonding network with nearby residues, Ala31 and Leu32 $[38,70]$. This hydrogen bonding network is believed to upregulates the redox potential of the heme iron as well as lowering the $\mathrm{pK}_{\mathrm{a}}$ of the distal ligand $[41,67]$. The redox potentials of $\mathrm{CPO}$ are highly $\mathrm{pH}$-dependent as the redox potential is $-140 \mathrm{mV}$ at $\mathrm{pH} 6.90$ and +150 $\mathrm{mV}$ at $\mathrm{pH} 2.70$ [71]. The $\mathrm{pH}$-dependency of the redox potential of $\mathrm{CPO}$ explains why this enzyme has an optimum $\mathrm{pH}$ at highly acidic level for chlorination, as the high redox potential requirement for oxidizing chloride during the reaction.

According to the crystal structure of $\mathrm{CPO}$, the distal region is composed of mostly polar residues such as Glu, Asp, and His, that resembles classical peroxidases. On the surface, two channels, one wide and one narrow, connects the surface to the heme center (Figure 1.8). Among the two channels, halide binding sites were only found in the narrow channel. However, halides might have diffused through the wide channel, where no binding site was observed in the crystal structure, to the heme center [70].

At the bottom of the two channels, they formed the substrate-binding pocket allowing the direct access to the heme. The substrate-binding pocket is formed by the hydrophobic residues consisting of Phe103, Leu70, Phe186, Val182, and Ala267. The sidechains of Phe103 and Phe186 are parallel to the heme plane and they are $6.2 \AA ̊$ away from each other. Phe103 has been shown to be flexible as the sidechain is displaced during the binding of the substrate to the distal pocket $[38,70]$. For P450, a substrate-binding pocket allows 
direct interaction between the substrate and the ferryl oxygen. However, heme peroxidases has restricted access to the ferryl oxygen and the substrate interacts with the enzyme through the heme edge. As for CPO, this enzyme utilizes both types of mechanisms depending on the types of reactions [58]. In the distal region, there is an acid-base catalyst responsible for the formation of the reactive species, Compound I. The acid-base catalyst responsible for the deprotonation of $\mathrm{H}_{2} \mathrm{O}_{2}$ and the cleavage of the $\mathrm{O}-\mathrm{O}$ bond is a glutamic acid residue (Glu183) for CPO while a histidine residue commonly found to plays this role in other peroxidases such as $\mathrm{CcP}$ (Figure 1.9) [72, 73]. The role histidine (His105) plays in CPO is subtler as to other peroxidase, where His 105 form a hydrogen bond with Glu183 and act as a proton shuttle during the cleavage of the peroxide bond [74].

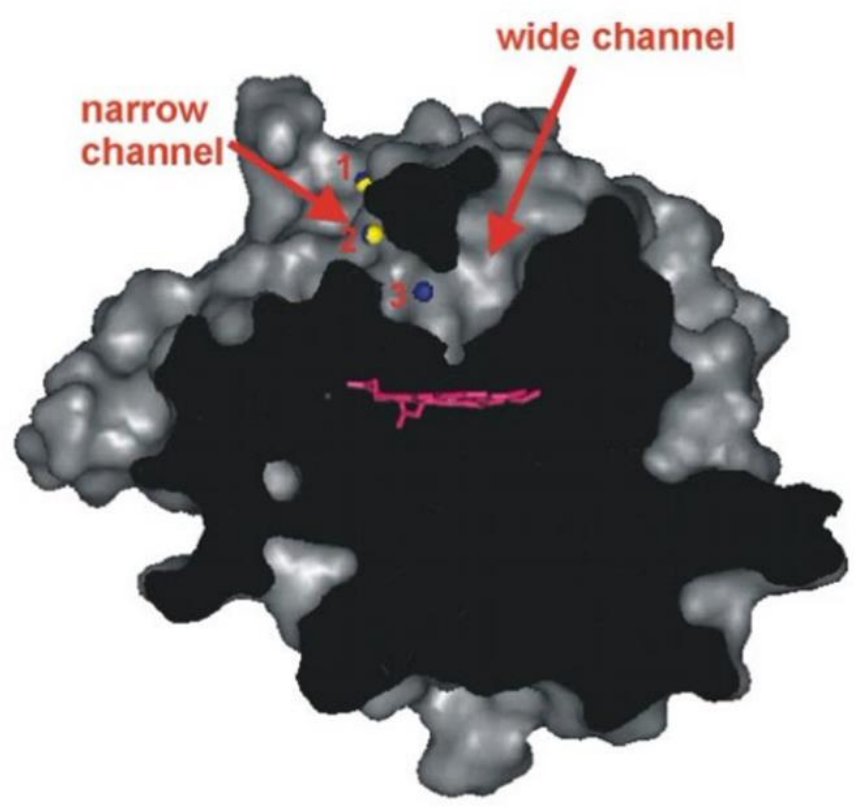

Figure 1.8 Slice through surface representation of CPO with the two channels connected to the active site [70]. Color representations: heme, magenta; bromide, yellow; iodide, blue. 

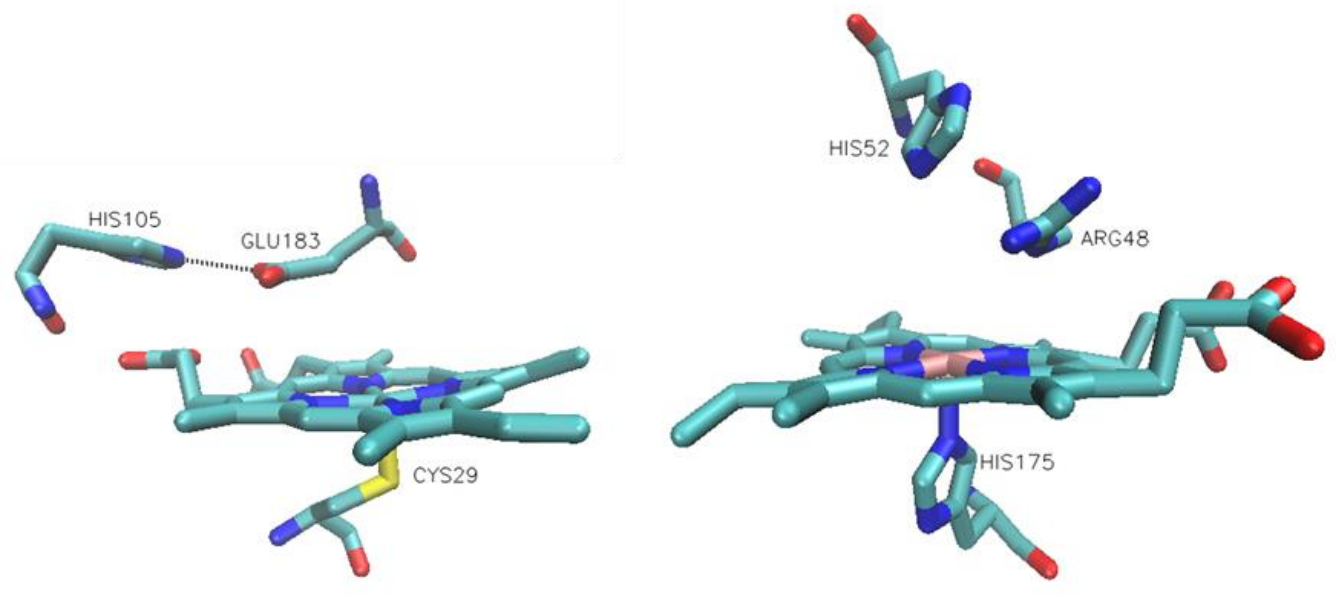

Figure 1.9 The acid-base catalyst of CPO(Left) and CcP(Right) are glutamic acid and histidine, respectively. The following PDB files were used to generate the figure: $1 \mathrm{CPO}$ and 2XIL

\subsubsection{Catalytic Cycle of CPO}

Compound I is defined as a oxo-ferryl radical species, where the radical can be located on either the porphyrin (such as HRP and $\mathrm{CPO}$ ) or nearby residue (such as $\mathrm{CcP}$ ), and is found throughout the catalytic cycle of heme enzymes. In term of CPO, the catalytic cycle began as a high-spin ferric state or resting state. The addition of hydrogen peroxide, oxidized the heme iron which results in the formation of Compound I, an oxo-ferryl porphyrin cation-radical. As shown in Figure 1.10, hydrogen peroxide binds to the ferric heme iron then the distal Glu183 deprotonates the proximal hydrogen of the peroxide forming the transient intermediate, $\mathrm{Fe}(\mathrm{III})-\mathrm{OOH}$, known as Compound 0. The protonated Glu183 will then protonates the distal oxygen of the peroxide as well as breaking the O-O bond leading to the release of water and the formation of Compound I $[75,76]$. 

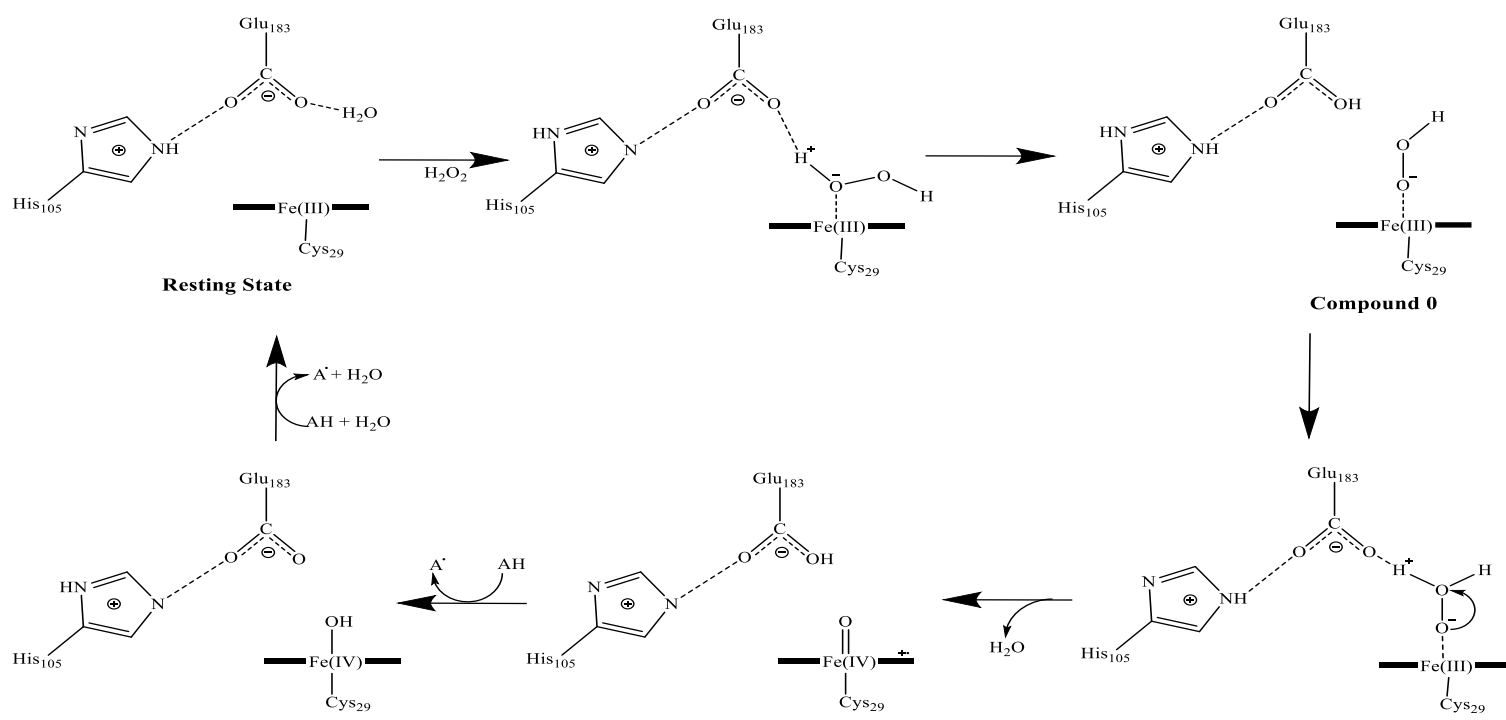

Compound II

Compound I

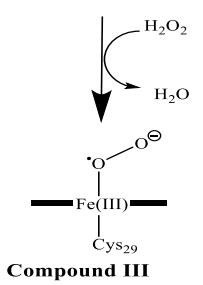

Figure 1.10 Proposed Mechanistic Formation of Compound 0, I, II, and III of CPO [77].

As one of the most versatile heme enzyme, Compound I of CPO can participate in different pathways catalyzing different reactions. As suggested by Dr. Hager, CPO-I can go through any of the four pathways which result in the return to its resting state (Figure 1.11). As peroxidase, CPO-I catalyzes two one-electron oxidations of organic molecules to a radical cation. After catalyzing the first one-electron oxidation, CPO-I is reduced to a second intermediate known as Compound II. Compound II of CPO or CPO-II is an oxoferryl species with one oxidation equivalent lower than CPO-I. X-ray absorption spectroscopic study on CPO-II by Dr. Green and his group revealed that the bond length of the Fe-O bond is $1.82 \AA$, which suggests the presence of a single bond instead of a double bond [78]. Therefore, CPO-II exists mainly in a protonated ferryl form with minor 
species as an unprotonated ferryl form. The second one-electron oxidation reaction returns CPO back to its resting state. However, CPO-II can react with the excess hydrogen peroxide generating the third intermediate known as Compound III (Figure 1.10). This intermediate is interconnected with the $\mathrm{H}_{2} \mathrm{O}_{2}$-dependent deactivation process of heme peroxidase as well as the possible involvement with the chlorination reaction catalyzed by $\mathrm{CPO}$ [72]. CPO-catalyzed peroxidation reaction has been proposed to occur predominantly outside of the heme active site as the substrate bind to the surface of the enzyme and mediated the electrons transfer through the heme edge (Figure 1.12, route b).

In halogenation reaction, halide ions were diffused into the active site through the narrow channel as there are multiple binding sites presented [70]. The halide ion reacts with CPO-I to form the ferric Fe-OCl- intermediate, Compound X (Figure 1.12, route a) [79] CPO-catalyzed chlorination is highly $\mathrm{pH}-$ dependent as the optimal $\mathrm{pH}$ is around 2.75 , and when the $\mathrm{pH}$ increases, the activity decreases dramatically. The reason for such a low $\mathrm{pH}$ optimum was explained in a study which demonstrated the chlorination of monochlorodimedone (MCD) to dichlorodimedone (DCD) was catalyzed by the protonated form of $\mathrm{Fe}-\mathrm{HOCl}$ instead of the unprotonated ferric $\mathrm{Fe}-\mathrm{OCl}$. The mechanism of the halogenation reaction catalyzed by $\mathrm{CPO}$ is still in controversial as whether the chlorination occurs within the heme active site or through a diffusible species. However, a proposed mechanism for chlorination of MCD is shown in Figure 1.13 [80]. 


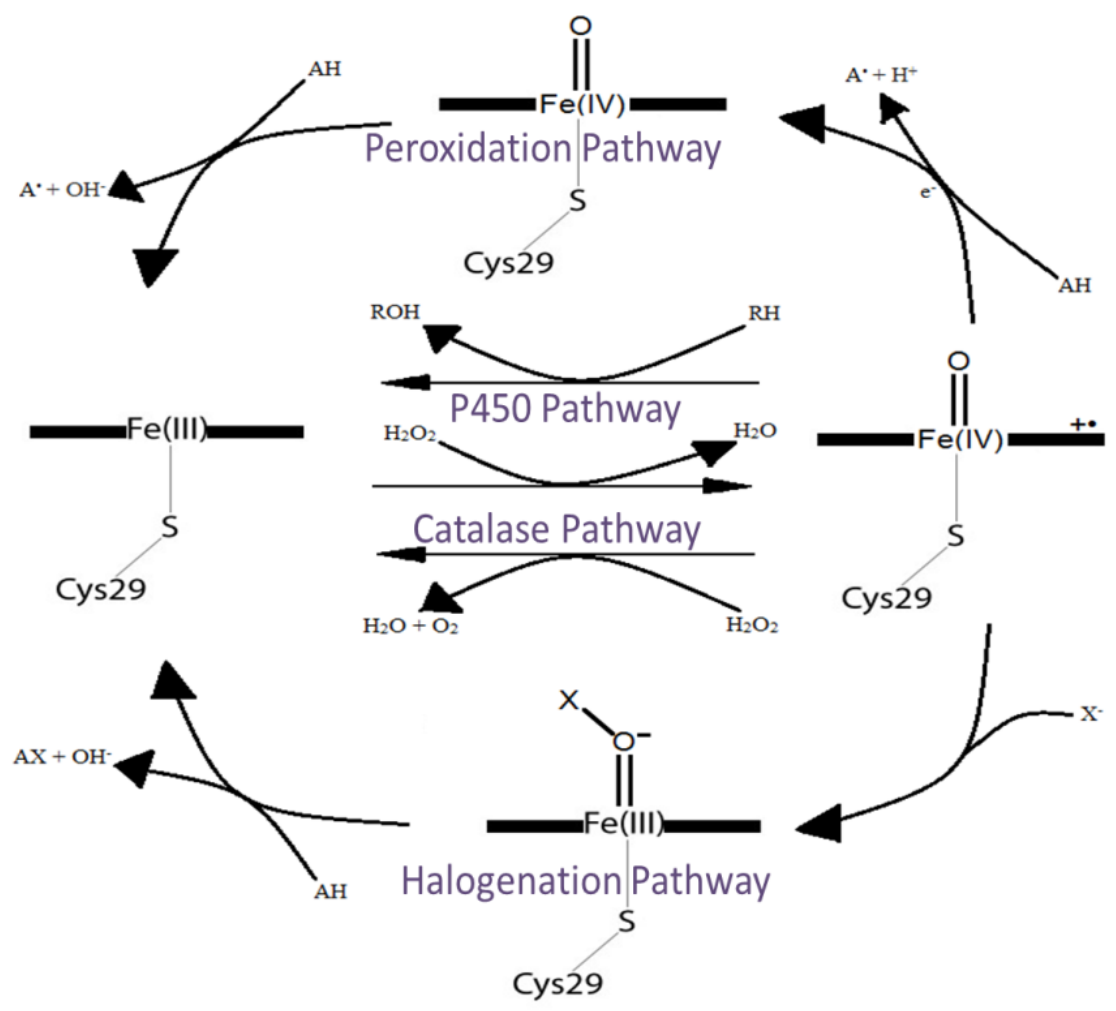

Figure 1.11 Catalytic cycle of CPO as suggested by Dr. Hager. R or RH represented organic substrate while $\mathrm{X}^{-}$represented a halide ion besides fluoride.

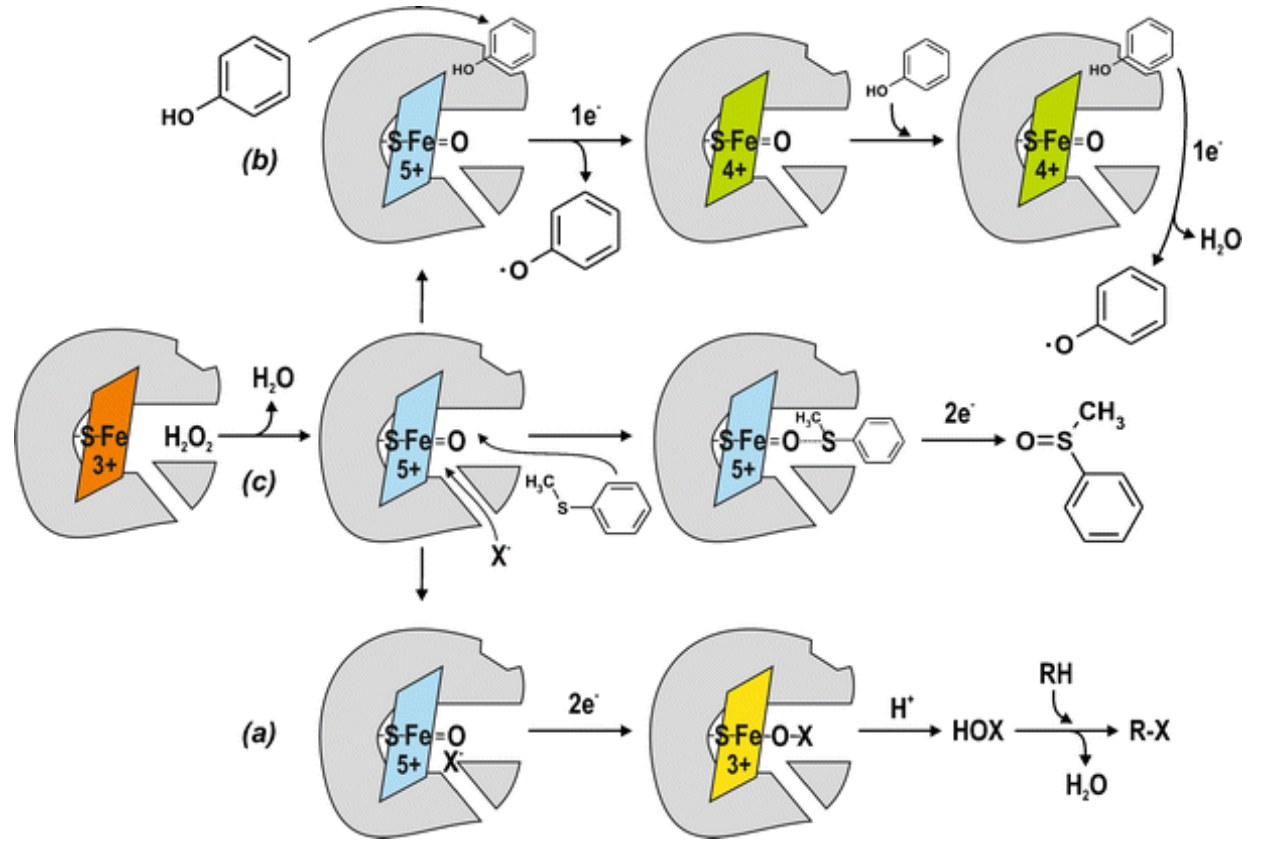

Figure 1.12 The simplified mechanistic route for CPO-catalyzed halogenation (a), peroxidation (b) and peroxygenation (c) reactions [81]. $R$ or $R H$ represented organic substrate while $\mathrm{X}^{-}$represented a halide ion besides fluoride. 


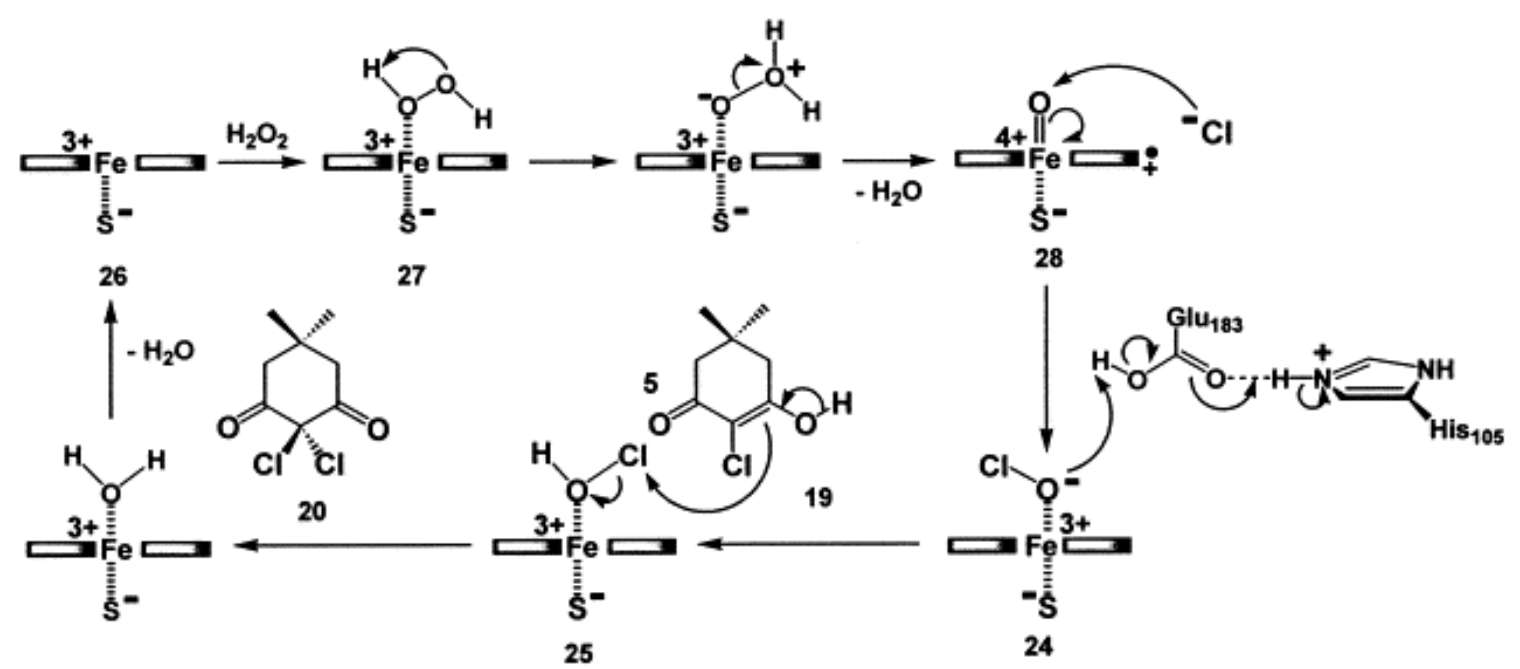

Figure 1.13 The proposed mechanism for chlorination of MCD catalyzed by CPO [80].

As a heme-thiolate protein, CPO catalyzed peroxygenation/oxygen-insertion reactions as a two-electron oxidation process similar to P450 enzymes. This type of reaction occurs within the active site of CPO (Figure 1.12, route c), as the reaction was hindered when all the histidine residues in the active site were alkylated [58]. The intermediate involved in CPO's catalysis is still unclear; however, the reaction might proceed through a dual oxygen-transfer mechanism that involve two different electrophilic species, oxoferryl species and iron hydroperoxide species, as illustrated in Figure 1.14 [82]. 
(a)

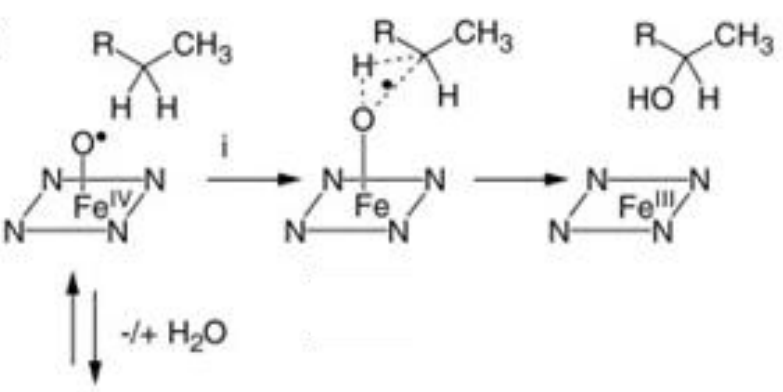

(b)

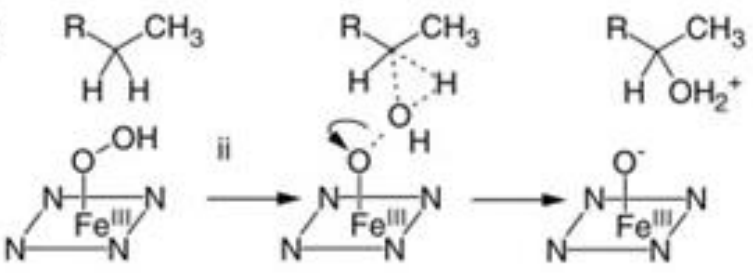

Figure 1.14 The proposed mechanism for oxygen-insertion reaction via a dual mechanism: (a) an oxoferryl species that inserts oxygen and (b) an iron hydroperoxide species that inserts $\mathrm{OH}^{+}$(Modified figure from van Rantwijk et al.) [82].

Another type of two-electron oxidation reaction catalyzed by $\mathrm{CPO}$ is the dismutation of hydrogen peroxide. In this route, CPO-I catalyzes the decomposition of a second molecule of hydrogen peroxide into dioxygen and water. On the basis of the Compound X involvement in halogenation pathway, the proposed mechanism of the catalase pathway might involve the formation of this CPO-I- $\mathrm{H}_{2} \mathrm{O}_{2}$ complex as illustrated in Figure 1.15 [83]. An acid-base catalyst, in the case of CPO, Glu183 will deprotonate the proximal oxygen and then protonate the distal oxygen of the peroxide, leading to the release molecular oxygen and water. However, this proposed mechanism seems to go through a two oneelectron transfer process instead of commonly assumed two-electron oxidation process [84]. Therefore, the exact mechanism of the catalase pathway of CPO is still unclear. 


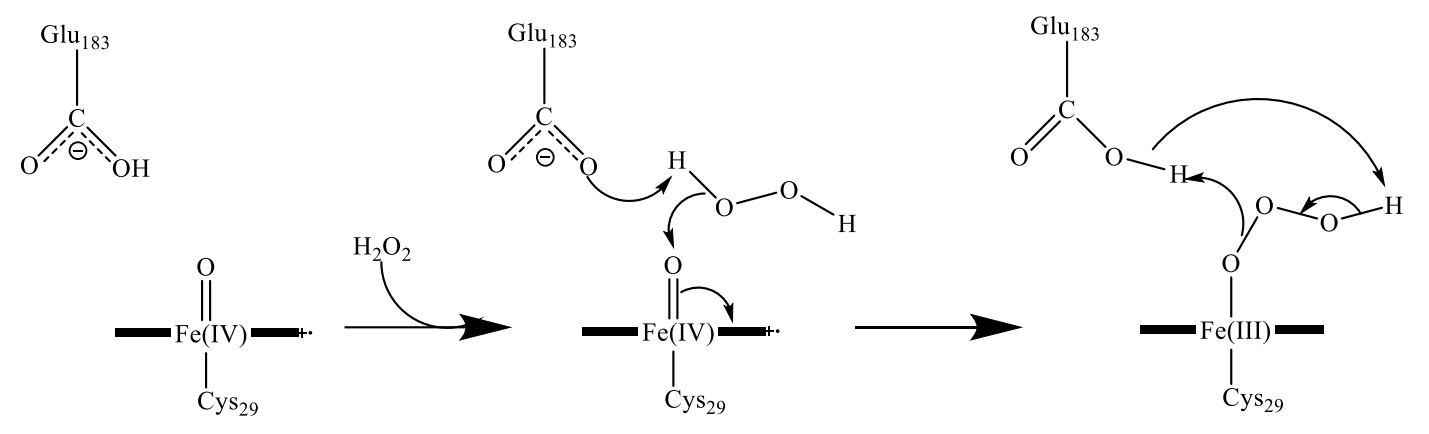

Compound I

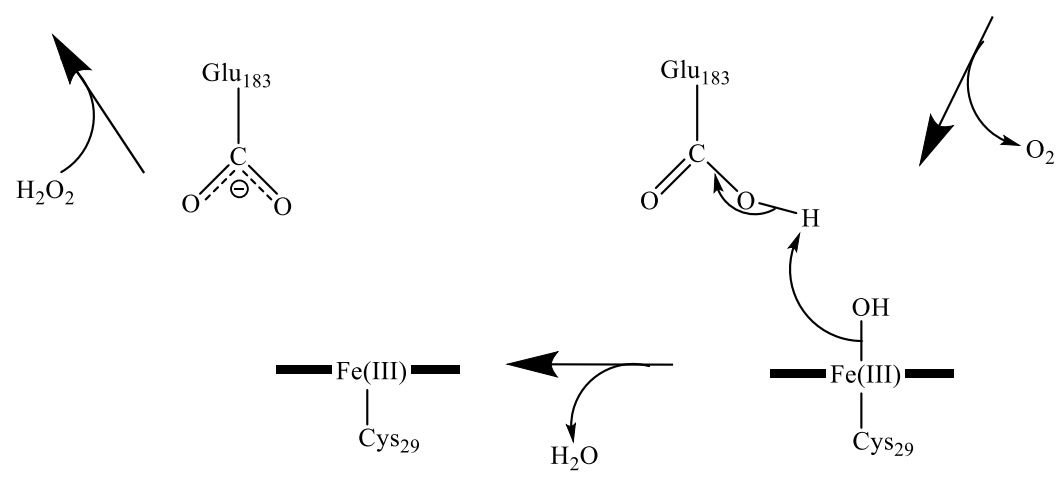

Figure 1.15 Proposed mechanism for dismutation of hydrogen peroxide catalyzed by CPO (Modified figure from Dr. Cécile Claude) [85]

\subsubsection{Significant of CPO-catalyzed reactions}

\subsubsection{Halogneation Reactions}

As previously stated, CPO is one of the most versatile heme enzyme capable of catalyzing different one-electron or two-electron oxidation reactions through different catalytic pathways. Possible substrates for CPO-catalyzed halogenation include alkenes [86-88], alkynes [89], anisole [90], $\beta$-diketones [33, 91], $\beta$-keto acid [55], phenols [92], and aromatic hydrocarbons [93]. Examples of halogenation reactions are shown in Figure 1.16. Many studies have suggested that $\mathrm{CPO}$ or $\mathrm{CPO}$-like enzymes are responsible for the natural production of organochlorine compounds found in the environment. CPO have been demonstrated to mediates the chlorination of polycylic aromatic hydrocarbons with 5 and 6 aromatic rings, and the aromatic groups in fulvic acid as well as the bromination of 
flavanones [57, 93-95]. Aromatic hydrocarbon pollutants can cause more severe damage as they can form these high molecular-weight halogenated compounds which are highly toxic to the environment and the formation of these compounds need to be fully understood to better protect the environment.

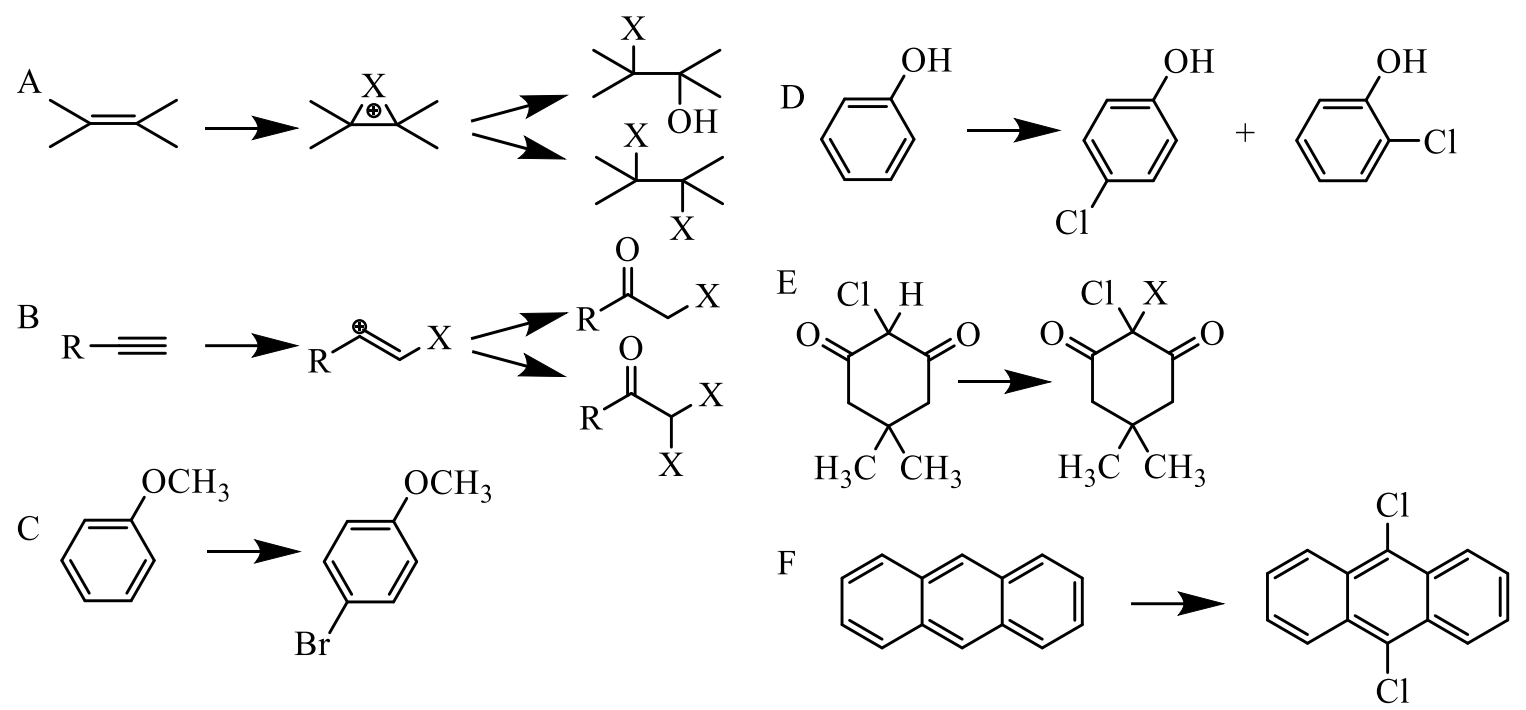

Figure 1.16 Halogenation reactions catalyzed by CPO: (A) alkene, (B) alkynes, (C) anisole, (D) phenol, (E) monochlorodimedone, (F) anthracene (aromatic hydrocarbon).

\subsubsection{Peroxidation Reactions}

Although CPO primary acts as a haloperoxidase, this enzyme can also catalyze oxidative dehalogenation of halophenols to less hazardous products, which implicates its possible application in bioremediation [96, 97]. Like other peroxidases, CPO can catalyze peroxidation reactions through two one-electron transfer processes. In the case of monohalophenols, 4-chlorophenol and 4-fluorophenol were significantly degraded by CPO, leading to the formation of quinones and biphenyl-like compounds [30, 98, 99]. Similar result was observed for pentachlorophenol, as CPO mediated the transformation of this substrate into a polymeric and insoluble material that is more readily remove from the 
environment [100]. The formation of dimers, trimers, and polymers have been proposed to be involved by the initial formation of phenolic radicals catalyzed by CPO involving the peroxidase's nature of this enzyme. An example is shown in Figure 1.17 [99]. This was confirmed by Osborne et al. in which the rapid scan stopped-flow spectroscopic spectral on the reactions of CPO-I and CPO-II with 2,4,6-trichlorophenol clearly revealed that the oxidative dehalogenation of TCP to 2,6-dichloro-1,4-bezoquinone occured through a two steps process. The proposed reaction scheme for oxidative dehalogenation of TCP catalyzed by CPO is shown in Figure $1.18[96,97]$. Interestingly, CPO is the only peroxidase that can catalyze halogenation and dehalogenation reactions with significantly higher dehalogenating activity than other peroxidases such as lignin peroxidase and HRP [101]. 
$10,12,13$<smiles>Oc1ccc(-c2ccc(O)c(F)c2)cc1F</smiles><smiles>Oc1ccc(-c2cccc(F)c2O)cc1F</smiles><smiles>Oc1c(F)cccc1-c1cccc(F)c1O</smiles><smiles>Oc1cc(F)c(Oc2cccc(F)c2)cc1Oc1cccc(F)c1</smiles><smiles>Oc1cccc(F)c1</smiles><smiles>[O-]c1cccc(F)c1</smiles><smiles>C=C=C1C=CC(=O)C(F)C1</smiles><smiles>C=CCCC</smiles><smiles>CC(C)CCCCCC(C)C(C)F</smiles><smiles>O=C1C=CC(=O)C=C1</smiles>
From 4-fluoro-<smiles>O=C1C=CC(Oc2ccc(F)cc2Oc2cc(F)cc(C3=C(c4cc(F)ccc4O)C(=O)C=CC3=O)c2OC2C=CC(=O)C=C2)C=C1</smiles>

Figure 1.17 Initial formation of phenolic radical from the oxidation of fluorophenol catalyzed by CPO and the possible products yielded [99]. 


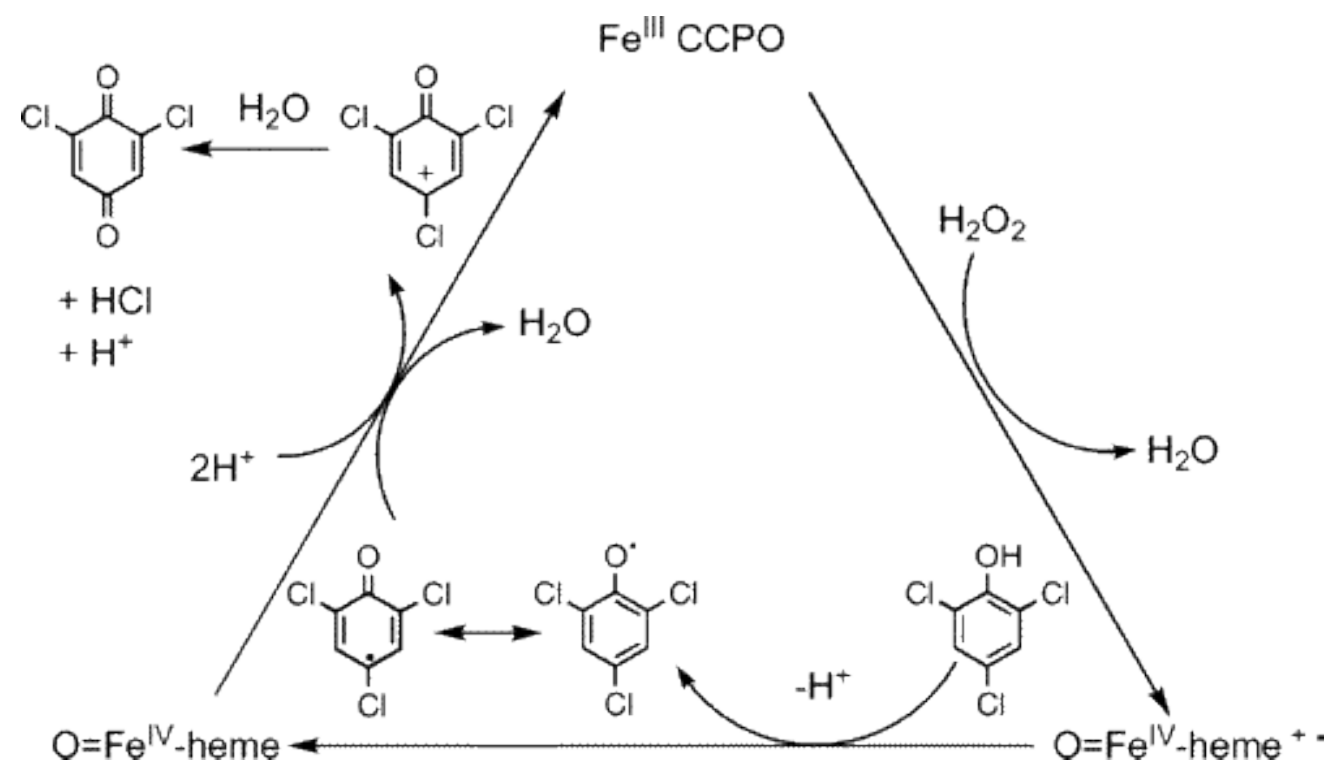

Figure 1.18 Two steps one-electron oxidative dehalogenation catalyzed by CPO involving CPO-I and CPO-II [97].

\subsubsection{Oxygen-insertion Reactions}

As stated in earlier section, CPO can catalyze P450-like or oxygen-insertion reactions. These reactions go through the regio- and enantioselective peroxygenation of the substrate, resulting the formation of prochiral or chiral products [30,31]. For example, CPO catalyzes sulfoxidation of sulfides such as alkyl aryl sulfides and $\beta$-carbonyl sulfides, to their corresponded sulfoxides with great yield as well as high enantiomeric excess, favoring the $(R)$-configuration as shown in Figure 1.19 [102-104]. In terms of bioremediation, organosulfides and thiophenes in diesel fuel can be oxidized by CPO yielding $(R)$ sulfoxides and sulfone that can be effectively remove through distillation [105]. The stereoselective manner of $\mathrm{CPO}$-catalyzed peroxygenation reactions has been a great interest to the pharmaceutical industry as enantiopure sulfoxides are important chiral synthons for asymmetric synthesis of natural products and biologically active compounds [106]. For example, CPO has demonstrated to synthesize $(R)$-modafinil through the 
enantioselective sulfoxidation of 2-(diphenylmethylthio) acetamide in an one step process with yield of $40.8 \%$ and the ee value of $97.3 \%$ [107].

(A)<smiles>CCCCCCCCCCCCCCC(C)=O</smiles>

(B)<smiles>CSCC(=O)c1ccccc1</smiles>

$100 \%$, ee $99 \%$

(C)<smiles>CCSCC(=O)c1ccc(C)cc1</smiles>

(D)<smiles>CSCC(=O)c1ccc(Cl)cc1</smiles>

(E)<smiles>CS[C@@H]1CCOC1=O</smiles>

$100 \%$, ee $95 \%$

Figure 1.19 CPO-catalyzed sulfoxidation of various sulfides with their corresponded sulfoxides yield and enantiomeric excess percentage [102, 103].

Another type of reaction catalyzed by CPO that has been a great interest in the field of pharmaceutical and synthetic chemical industries is the epoxidation reaction. Many studies revealed that $\mathrm{CPO}$ catalyzes epoxidation of cis-disubstituted, 1,1-disubstituded, and 
trisubstituted alkenes with less than 8 carbons with great yield and ee. However, transolefins, and terminal and mono-substituted alkenes are poor substrates for CPO. Majority of CPO-catalyzed epoxidation reactions yielded in $(R)$-configuration epoxides as shown in Figure 1.20 [108-110]. These chiral epoxides have served as drug intermediates and the enzymatic synthesis of these compounds have shown to be more environmental friendly and cost-efficient relative to the organic synthesis methods. Practical examples such as CPO-assisted synthesis of $(R)$-(-)-mevalonolactone and $(R)$-dimethyl 2-methylaziridine1,2-dicarboxylate showed great efficiency $[111,112]$. The disadvantage of CPO is the presence of the catalatic activity, depleting the amount of oxidant such as hydrogen peroxide for the formation of Compound I. The replacement of the hydrogen peroxide with tert-butyl peroxide as oxidant was shown to have a better yield and enhanced the turnover number for the CPO $[113,114]$.

(A)

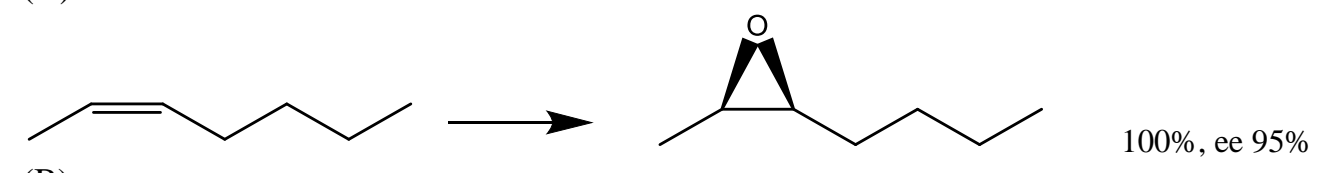

(B)

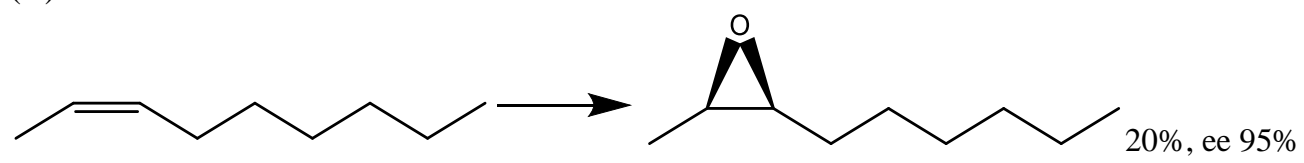

(C)

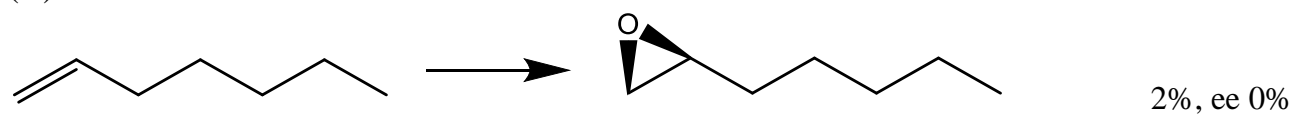

(D)<smiles>C(=Cc1ccccc1)c1ccccc1</smiles>

$40 \%$, ee $49 \%$

Figure 1.20 CPO-catalyzed epoxidation of various (A-C) olefins and (D) styrene to their corresponded epoxides yield and enantiomeric excess percentage [108]. 
Epoxidation and sulfoxidation both occur in a stereoselective manner with favoring of the $(R)$-configuration. However, hydroxylation is a type of oxygen-insertion reactions that can be stereoselective or nonstereoselective depending on the nature of the substrate. For example, CPO-catalyzed oxidation of 1,3-cyclohexadienes and 1,3-cyclohexadiene diacetate resulted in a product with an ee of $78 \%$ and a racemic mixture, respectively. Possible substrates for CPO-catalyzed hydroxylation are acetylenes [115], cyclic conjugated dienes[116, 117], p-methyl and p-ethyl anisole as well as others benzylic compounds [108, 118], and monoterpenes [119] and examples are shown in Figure 1.21. Interestingly, CPO can hydroxylate ethylbenzene and propylbenzene to $(R)$-2-phenethyl alcohol and (S)-1-phenyl-1-propanol, respectively [108]. Even though they yielded different configurations, both reactions yielded a high ee value of greater than $88 \%$. Another factor that contribute to the stereoselectivity of the hydroxylation reactions is the condition of the reaction system. As observed for the oxidation of $R$-(+)-limonene, the presence of $\mathrm{KCl}$ and absence of $\mathrm{KCl}$ yielded in different degree of stereoselectivity as observed in Figure 1.21 (D) [119]. In the bracket of C and D in Figure 1.21, the proposed epoxide intermediates were spontaneously hydrolyzed to form the hydroxylated products. In addition, further oxidation of alcohols to aldehydes were observed for CPO-catalyzed hydroxylation reactions $[120,121]$. For example, CPO can catalyze the oxidation of amino alcohol during the synthesis of $\mathrm{N}$-Cbz-3-aminopropanal [122]. The versatility of CPO illustrated the potential of this biocatalyst for practical applications; however, the mechanisms for these reactions are still in controversial. Therefore, the study on the structure-function relationship of $\mathrm{CPO}$ is essential for practical application of this biocatalyst and would further our understanding on heme-thiolate proteins. 
(A)<smiles>CCC#CCCCCCC#C[C@@H](C)O</smiles>

(B)<smiles>CCc1ccccc1</smiles>

(C)<smiles>O[C@H]1C=C[C@H](O)CC1</smiles>

(D)

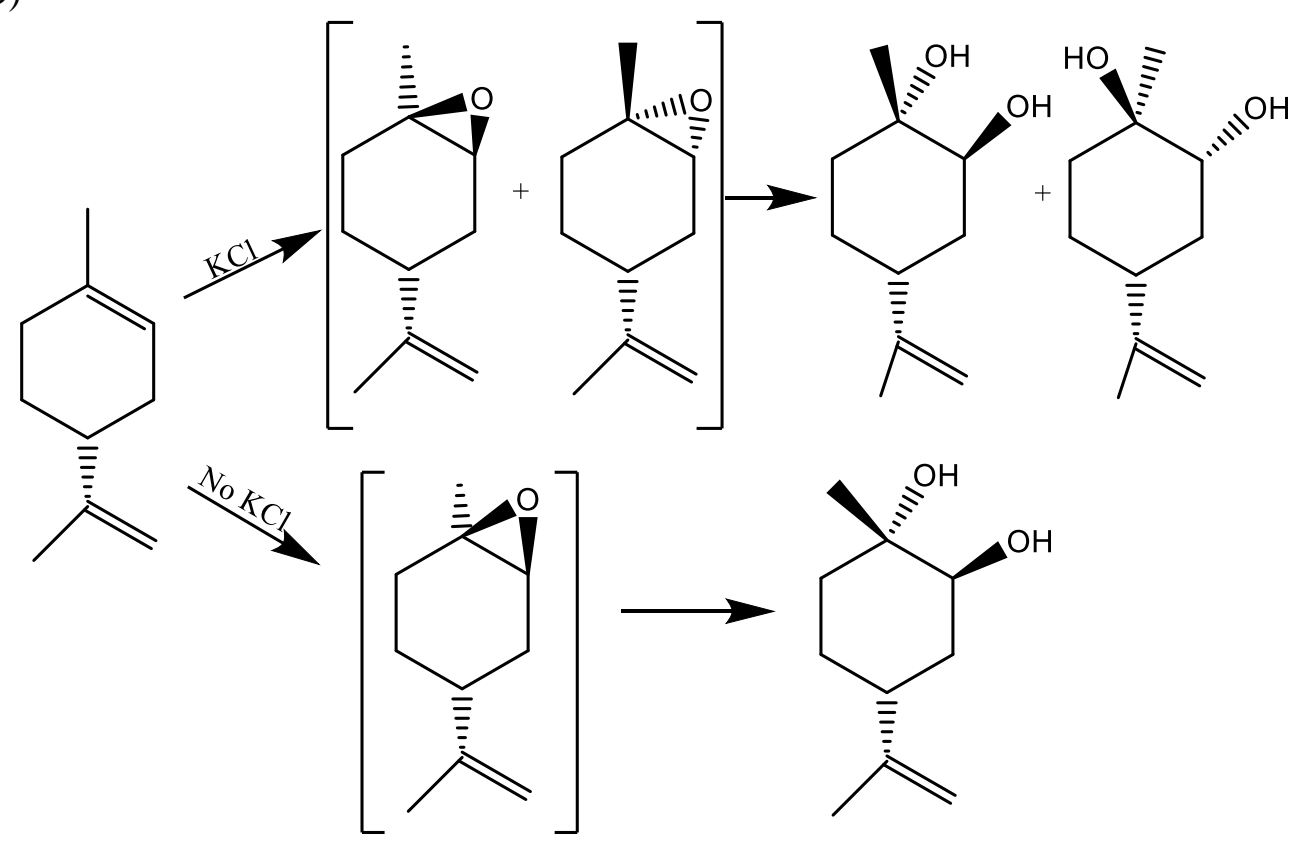

Figure 1.21 CPO-catalyzed progargylic hydroxidation (A), benzylic hydroxylation (B), cyclic conjugated diene hydroxylation $(C)$ and $R-(+)$-limonene hydroxylation in the presence and absence of $\mathrm{KCl}$ [108, 115-118].

\subsubsection{Mutagenesis Approaches on CPO}

To investigate the structure-function relationship of CPO, many site-directed mutagenesis studies were previously performed. The studies focused on the distal pocket 
of CPO are summarized in Table 1.1. Most of the distal CPO mutants shared similar catalytic activities as the chlorination and dismutation activities were diminished while the epoxidation and peroxidation activities were enhanced. Similar activity changes for the distal CPO mutants were observed for the proximal CPO mutants as listed in Table 1.2. My research investigated the effects of the proximal helix as well as the distal substratebinding pocket on the structural and biochemical properties of CPO. In addition, we investigated the importance of the distal pocket on modulating the enantioselectivity of styrene derivative substrates.

Table 1.1 The effects of the distal environment of CPO on its catalytic activities.

\begin{tabular}{|l|l|l|}
\hline Distal CPO Mutants & Activity & References \\
\hline N74V & $\begin{array}{l}\text { Increased in epoxidation activity, decreased } \\
\text { in chlorination and dismutation activities }\end{array}$ & {$[123]$} \\
\hline F103A & $\begin{array}{l}\text { No sign of chlorination and dismutation } \\
\text { activities, increased in epoxidation and } \\
\text { peroxidation activities }\end{array}$ & {$[124]$} \\
\hline H105A & $\begin{array}{l}\text { Increased in epoxidation activity, decreased } \\
\text { in chlorination and dismutation activity }\end{array}$ & {$[123]$} \\
\hline E183H & $\begin{array}{l}\text { Increased in epoxidation activity, decreased } \\
\text { in chlorination and dismutation activities }\end{array}$ & {$[125]$} \\
\hline F186A & $\begin{array}{l}\text { No sign of chlorination activity, decreased } \\
\text { in dismutation activities, and increased in } \\
\text { epoxidation and peroxidation activities }\end{array}$ & This work \\
\hline F103A/F186A & $\begin{array}{l}\text { No sign of chlorination activity, decreased } \\
\text { in dismutation activities, and increased in } \\
\text { epoxidation and peroxidation activities }\end{array}$ & This work \\
\hline
\end{tabular}


Table 1.2 The effects of the proximal helix of CPO on its catalytic activities.

\begin{tabular}{|c|c|c|}
\hline Proximal CPO Mutant & Activity & References \\
\hline $\mathrm{R} 26 \mathrm{~A}$ & $\begin{array}{l}\text { No sign of chlorination activity, decreased } \\
\text { in dismutation activity, increased in } \\
\text { epoxidation activity }\end{array}$ & {$[126]$} \\
\hline $\mathrm{P} 28 \mathrm{~A}$ & $\begin{array}{l}\text { No sign of chlorination and dismutation } \\
\text { activities, increased in epoxidation and } \\
\text { peroxidation activities }\end{array}$ & This work \\
\hline $\mathrm{C} 29 \mathrm{H}$ & $\begin{array}{l}\text { Retained similar catalytic activities as WT- } \\
\text { CPO } \\
\text { No sign of chlorination and dismutation } \\
\text { activities, increased in epoxidation and } \\
\text { peroxidation activities }\end{array}$ & $\begin{array}{l}{[127]} \\
{[126]}\end{array}$ \\
\hline $\mathrm{C} 29 \mathrm{H} / \mathrm{C} 79 \mathrm{H} / \mathrm{C} 87 \mathrm{H}$ & $\begin{array}{l}\text { Increased in epoxidation activity, } \\
\text { decreased in chlorination and dismutation } \\
\text { activities }\end{array}$ & [126] \\
\hline $\mathrm{P} 30 \mathrm{~A}$ & $\begin{array}{l}\text { No sign of chlorination activity, decreased } \\
\text { in dismutation activities, and increased in } \\
\text { epoxidation and peroxidation activities }\end{array}$ & This work \\
\hline $\mathrm{P} 28 \mathrm{~A} / \mathrm{P} 30 \mathrm{~A}$ & $\begin{array}{l}\text { No sign of chlorination and dismutation } \\
\text { activities, increased in epoxidation and } \\
\text { peroxidation activities }\end{array}$ & This work \\
\hline $\mathrm{N} 33 \mathrm{~A}$ & $\begin{array}{l}\text { No sign of chlorination and dismutation } \\
\text { activities, increased in epoxidation and } \\
\text { peroxidation activities }\end{array}$ & [126] \\
\hline R26A/N33A & $\begin{array}{l}\text { No sign of chlorination and dismutation } \\
\text { activities, increased in epoxidation and } \\
\text { peroxidation activities }\end{array}$ & [126] \\
\hline
\end{tabular}




\section{CHAPTER II.}

\section{INFLUENCE OF THE PRO-CYS-PRO TRIPEPTIDE IN THE PROXIMAL LOOP ON THE SPECTROSCOPIC PROPERTIES OF CHLOROPEROXIDASE}

\subsection{Background}

The active site of CPO can be divided into the distal and proximal regions by the heme center. As stated in the previous chapter, the distal pocket of $\mathrm{CPO}$ contains residues that interact with the substrate, regulating the stereoselectivity of the reaction. The catalytic heme center is covalently bound to the proximal ligand, cysteine, which is located on the beginning of helix A. Within the proximal pocket of $\mathrm{CPO}$, there is a hydrogen bonding network form between helix A and the nearby loop, Arg26-Asn37 and Ala27-Asn33, as observed in Figure 2.1. This hydrogen bonding network is previously studied in our research lab and shown to play an important role in maintaining the secondary and tertiary structure of CPO as well as other spectroscopic properties of the enzyme [126]. In CPO, another type of hydrogen bonding network is also found in the proximal region, which is the amide-sulfur hydrogen bonding network. This type of hydrogen bonding network is conserved throughout heme-thiolate proteins as observed in P450 enzymes, NOS and APOs $[31,53,80]$. In P450cam, the proximal region contains three amide-sulfur hydrogen bonds between the axial ligand, Cys357, and the adjacent residues, Leu358, Gly359, and Gln360. As for CPO, the sulfur atom of Cys29 form two amide-sulfur hydrogen bonds with the backbone amide of Ala31 and Leu32. In CPO, Cys29 residue is sandwiched by two proline residues, Pro28 and Pro30, and this tripeptide stretch is superimposed to other heme-thiolate proteins. In addition, this Pro-Cys-Pro tripeptide stretch is shown to be

conserved in APOs as seen in Figure 1.7. The number of amide-sulfur hydrogen bonds in 
$\mathrm{CPO}$ is different than P450cam because of the adjacent residues of the proximal cysteine in $\mathrm{CPO}$ are prolines, which limit the access to the backbone amide [38]. To examine the importance of these proline residues in $\mathrm{CPO}$ as well as other heme-thiolate proteins, Pro28 and Pro30 were mutated to an Ala residue to examine their effects on the spectroscopic properties of CPO.

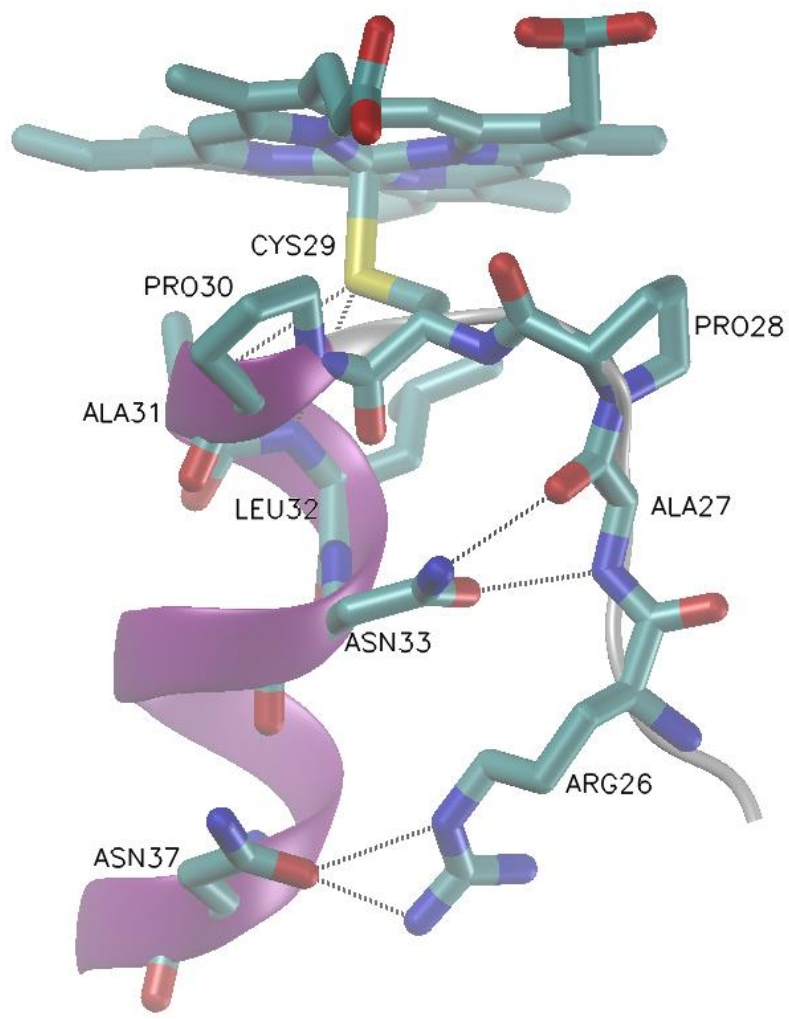

Figure 2.1 The hydrogen bonding networks in the proximal pocket of CPO.

Ultraviolet-Visible spectrophotometry can examine the electronic structural properties of the catalytic heme center and its surrounding. A typical UV-Vis spectrum of a heme protein is distinguished by the two regions, near-ultraviolet and visible regions. These two regions corresponded to two type of bands which are the Soret or B band and the Q-bands. The Soret band originated from the transition of the ground state to the second exited state that absorb in between 380-500 $\mathrm{nm}$. As for the Q-bands, the weak transition from the 
ground state to the first excited state was observed and occasionally, multiple absorption peaks between 500-750 $\mathrm{nm}$ are observed [128]. The change or shift of these absorption peaks indicates the change in the heme environments such as the coordination and the spin state of the heme iron. Therefore, the comparison of WT-CPO and the CPO mutants will provide valuable insight on the effects of these proline residues on the enzyme's electronic properties. Besides UV-Vis spectrophotometry, circular dichroism has been commonly used to estimate the protein's secondary structure composition and to observe structural changes in different conditions. Spectra's data can be analyzed by different programs such as CONTINLL, SELCON3, CDSSTR, VARSLC and K2D to estimate the secondary structure [129].

\subsubsection{Aim of this study}

In the present study, Pro28 and Pro30 were mutated to an Ala residue through sitedirected mutagenesis. Three CPO mutants, P28A-, P30A-, and P28A/P30A-CPO, were expressed and purified for spectroscopic characterization relative to WT-CPO. This investigation revealed the importance of this conserved Pro-Cys-Pro stretch or the function of these proline residues in the proximal loop of $\mathrm{CPO}$ which is applicable to other hemethiolate proteins for maintaining its structural properties.

\subsection{Materials and Methods}

\subsubsection{Materials}

The expression of the CPO mutant take place through the MGG029 strain of Aspergillus niger and this strain was purchased from America Type Culture Collection (ATCC) (Manassas, VA). The expression vector, pCPO3.I-Amds, and co-transformation plasmid, pAB4.1, containing PyrG selection marker were obtained from TNO 
Microbiology and Systems Biology, Netherlands. The site-specific mutagenesis of pCPO3.I-Amds was performed through the QuikChange II XL site-directed mutagenesis kit and Q5 site-directed mutagenesis kit from Stratagene (La Jolla, CA) and New England BioLabs (Ipswich, MA), respectively. Restriction enzymes were also purchased from New England BioLabs (Ipswich, MA). Purified oligonucleotide primers were purchased from Eurofins Genomics (Louisville, KY). De-ionized water for all experiments were prepared freshly by Milli-Q Biocel (Millipore, Billerica, MA). All other chemicals and materials were purchased from Fisher Scientific (Waltham, MA) unless otherwise stated.

\subsubsection{Construction of P28A-, P30A-, and P28A/P30A-CPO genes}

The pCPO3.1-AmdS vector was used for site-directed mutagenesis (Figure 2.2). This expression vector was encoded with a full-length $\mathrm{CPO}$ coding sequence controlled by an Aspergillus niger glucoamylase promoter, Aspergillus nidulans trpC terminator, pUC19 vector sequence, and an A. nidulans AmdS selection marker. The pUC19 vector sequence allows multiple-copy production in Escherichia coli and A. nidulans AmdS selection marker enable selection in polyacrylamide plate. The mutation(s) were introduced through polymerase chain reaction amplification using the mutagenesis kits listed in the previous section. The PCR reactions were carried out in Eppendorf thermal cycler (Mastercycler gradient). The compositions of the PCR reaction are listed in Table 2.1. The primers used for the PCR reactions are listed in Table 2.2. 
Table 2.1 PCR reaction's composition.

\begin{tabular}{|l|l|}
\hline Reagent & Amount \\
\hline Q5 Hot Start High-Fidelity 2X Master Mix & $12.5 \mu \mathrm{L}$ \\
\hline Forward Primer & $125 \mathrm{ng}$ \\
\hline Reverse Primer & $125 \mathrm{ng}$ \\
\hline Template DNA & $1-25 \mathrm{ng}$ \\
\hline DI-Water & $\mathrm{x} \mu \mathrm{L}($ Final Volume: $25 \mu \mathrm{L})$ \\
\hline
\end{tabular}

Table 2.2 CPO mutant's primers.

\begin{tabular}{|l|l|}
\hline Proximal Mutant CPO & Primers \\
\hline P28A-sense & 5'GACTCTCGTGCTGCCTGCCCAGCT3' \\
\hline P28A-antisense & 5'AGCTGGGCAGGCAGCACGAGAGTC3' \\
\hline P30A-sense & 5'CGTGCTCCTTGCGCCGCTCTGAACGCT3' \\
\hline P30A-antisense & 5'AGCGTTCAGAGCGGCGCAAGGAGCACG3' \\
\hline P28A/P30A-sense & 5'CTCTCGTGCTGCCTGCGCCGCTCTGAACGCT3' \\
\hline P28A/P30A-antisense & 5'GGATGTGTCGTGCTCGGCAGCGTGGGGCTCGGC3' \\
\hline
\end{tabular}
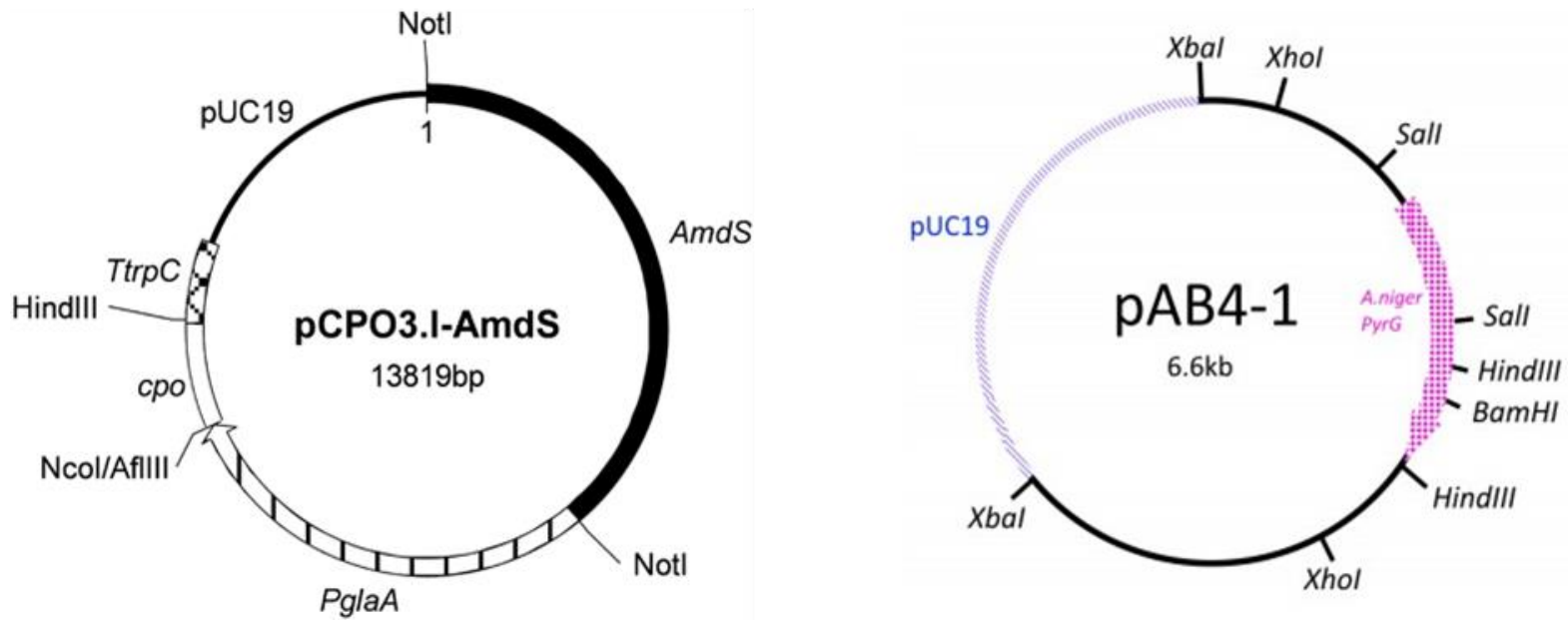

Figure 2.2 Plasmids used in fungal transformation. (A) pCPO3.I-AmdS plasmid containing A. niger glucoamylase promoter (PglaA), CPO gene from $C$. fumago, $A$. nidulans anthranilate synthetase terminator (TtrpC), and $A$. nidulans acetamidase gene (AmdS). (B) pAB4-1 plasmid containing A. niger PyrG gene and pUC 19 vector. 
The PCR condition for each cycle is $98.0^{\circ} \mathrm{C}$ denaturation for 50 seconds, $60.0^{\circ} \mathrm{C}$ annealing for 50 seconds, and $72.0^{\circ} \mathrm{C}$ extension for 14 minutes. A total of 25 cycles were performed with the final extension of the reaction $72.0^{\circ} \mathrm{C}$ extension for 7 minutes then the reaction was held at $4.0^{\circ} \mathrm{C}$. The PCR product was digested with Dpn I restriction endonuclease to remove the methylated DNA template leaving behind the mutated DNA. The PCR product was transformed into Escherichia coli (Dh5 $\alpha$ strain) and plated on LBcarbenicillin agar plates for selection. Each mutation was confirmed through DNA sequencing and restriction enzymes digestion along with electrophoresis (Figure 2.3 and 2.4).

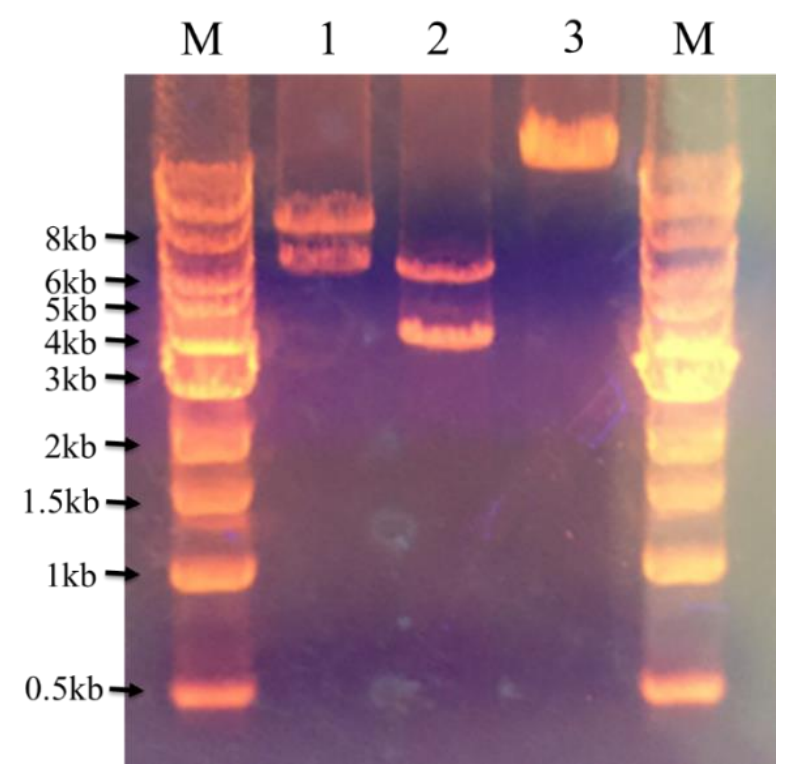

Figure 2.3 DNA Electrophoresis of Restriction digestion of pCPO3.I-AmdS. Lane 1undigested DNA, Lane 2-NotI, Lane3-HindIII. 

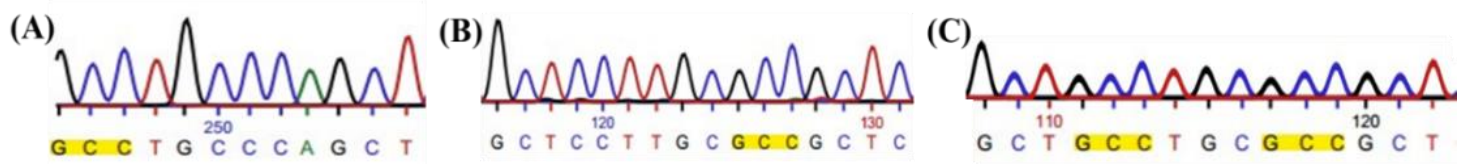

Figure 2.4 DNA Sequencing of (A) P28A-, (B) P30A-, and (C) P28A/P30A-CPO

\subsubsection{Transformation of mutant plasmid into Aspergillus niger}

The mutant plasmid is transformed into A. niger MGG029 strain along with pAB4.1 through the previously described procedure with the following modifications [56]. Brown conidiophores of MGG029 were inoculated in 50-mL minimal medium (MM) containing $0.5 \%$ yeast extract, $0.5 \%$ Casamino acids, $5 \%$ maltose, $1 \%$ glucose, $70 \mathrm{mM} \mathrm{NaNO}_{3}, 7 \mathrm{mM}$ $\mathrm{KCl}, 6 \mathrm{mM} \mathrm{KH}_{2} \mathrm{PO}_{4}, 6 \mathrm{mM} \mathrm{K}_{2} \mathrm{HPO}_{4}, 2 \mathrm{mM} \mathrm{MgSO}_{4}, 10 \mathrm{mM}$ uridine, $25 \mu \mathrm{L}$ of carbenicillin (100 mg/mL stock), $50 \mathrm{uL}$ of the Hunter trace elements solution. The $50-\mathrm{mL}$ culture was incubated at $37^{\circ} \mathrm{C}$ and $250 \mathrm{rpm}$ for $24-48$ hours then the temperature is lowered to $30^{\circ} \mathrm{C}$ for 48-72 hours until white-yellow mycelium is observed. Afterward, the 50-mL culture is disrupted and transferred to $1-\mathrm{L} \mathrm{MM}$ in a $2 \mathrm{~L}$ flask. The 1-L culture was continued to be incubated in the same condition for another 16-20 hours. The mycelium was filtered through sterile miracloth and resuspended in $1.7 \mathrm{Osm}$ solution $(270 \mathrm{mM} \mathrm{CaCl} 2$ and 600 $\mathrm{mM} \mathrm{NaCl}$ ) in $1 \mathrm{~g}$ of mycelium per $20 \mathrm{~mL}$. The resuspended protoplasts were digested by $150 \mathrm{mg}$ lysing enzyme Sigma L-1412 for $4.0-4.5$ hours at $150 \mathrm{rpm}$ and $37^{\circ} \mathrm{C}$. The digested protoplasts were resuspended and incubated on ice for 20 minutes. The digested protoplasts were collected through centrifugation $\left(3500 \mathrm{rpm}, 15\right.$ minutes, $\left.0^{\circ} \mathrm{C}\right)$ and washed twice with STC $1700\left(1.2 \mathrm{M}\right.$ sorbitol, $10 \mathrm{mM}$ Tris- $\mathrm{HCl}$ at $\left.\mathrm{pH} 7.50,50 \mathrm{mM} \mathrm{CaCl}_{2}, 35 \mathrm{mM} \mathrm{NaCl}\right)$. The protoplasts were resuspended in the leftover of STC1700 during the washing process. The protoplast suspension is mixed with $10 \mu \mathrm{g}$ mutant plasmid and $1 \mu \mathrm{g}$ of pAB4.1 then the 
solution was incubated at $25^{\circ} \mathrm{C}$ for 25 minutes. The DNA-protoplast mixture was mixed with $60 \%$ PEG4000 in $10 \mathrm{mM}$ Tris- $\mathrm{HCl}$ at $\mathrm{pH} 7.50$ and $50 \mathrm{mM} \mathrm{CaCl}_{2}$ and then incubated at $25^{\circ} \mathrm{C}$ for 20 minutes. The polyethylene glycol-treated protoplast suspensions were diluted by 5-10 mL of STC1700. The protoplasts were collected and resuspended with STC1700. The suspension was plated on 1.2 M sorbitol acrylamide selective plate and successful transformants were used for protein expression [55].

\subsubsection{Expression and Purification of P28A-, P30A-, and P28A/P30A-CPO.}

The colonized acrylamide plates were cultured in MM lacking uridine, yeast extract, maltose, and glucose containing $10 \%$ fructose and $100 \mu \mathrm{M} \delta$-aminolevulinic acid. Cultures were incubated in a rotary shaker at $250 \mathrm{rpm}$ and $25^{\circ} \mathrm{C}$ for 2 days. On the third day, the temperature was lowered to $22^{\circ} \mathrm{C}$ (the optimal temperature for the $\mathrm{CPO}$ mutant expression), for 5additional days. Different assays, described in the later section, were used to monitor the production of the CPO mutant(s).

The cultures were filtered through Fisherband glass fiber filter circles G6. The filtrate was concentrated to $30 \mathrm{~mL}$ through Amicon high-pressure cells with $30 \mathrm{kDa}$ filter membrane cutoffs. After concentrating the medium, the sample was dialyzed against 4L buffer A (25 mM phosphate buffer, pH 5.90) overnight with at least one-time change of buffer. The sample was filtered through $0.45 \mu \mathrm{m}$ filter and subjected to two types of chromatography, ion-exchange and size exclusion.

For ion-exchange, the column was packed with diethylaminoethanol (DEAE) sepharose and equilibrate with buffer A. The sample was injected into the column and washed with buffer A while gradually increasing, 0-0.50 M, $\mathrm{NaCl}$ concentration. When the protein eluted, a dark green/red color is observed. Eluted fractions with Reinheitzahl (Rz)- 
(A420/A280) values above 0.7 were combined and concentrated to approximately $1 \mathrm{~mL}$ through the Millipore 30,000 Da centrifugal filter unit. The concentrated fractions were further purified through the size exclusion column packed with Sephadex G75 resin. Similarly, the eluted fractions with the highest $(\mathrm{Rz} \approx 1.2-1.4) \mathrm{Rz}$-value were combined and concentrated for analysis (Figure 2.5).

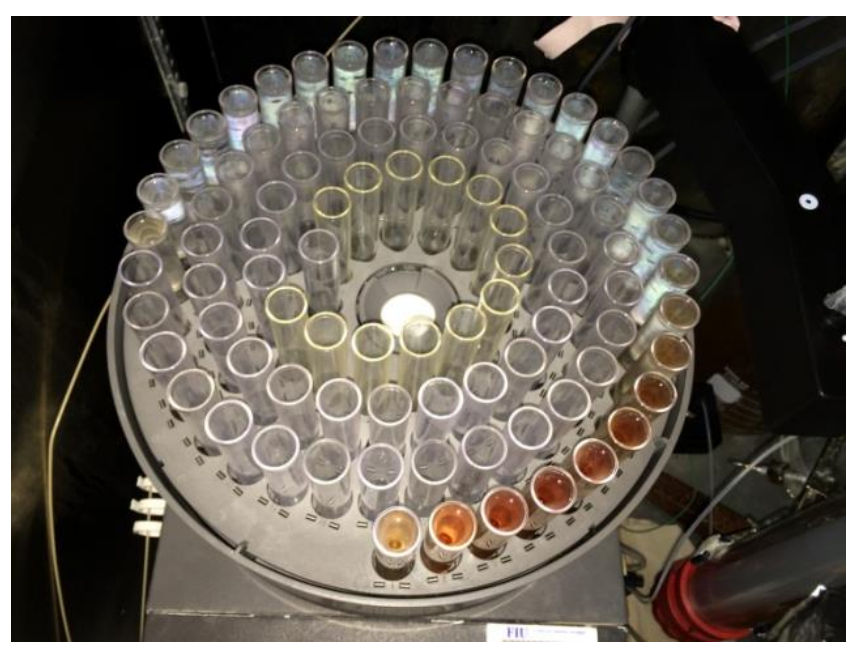

Figure 2.5 Eluted fractions collected after gel-filtration chromatography.

\subsubsection{UV-Visible spectroscopic characterization of CPO mutants}

A VARIAN UV-Vis spectrophotometer (Cary 300 Bio) was used for the all the UVVis spectroscopic analysis of CPO. The UV-Vis absorption spectra of WT-CPO and the $\mathrm{CPO}$ mutants were taken in $50 \mathrm{mM}$ phosphate buffer at $\mathrm{pH} 2.50-6.00$ throughout the wavelength of 200-800 $\mathrm{nm}$ using 1-cm quartz cuvettes.

\subsubsection{Ligand Binding Study}

Absorption spectra was taken for mutant and WT-CPO in the absence and presence of exogenous ligands, $\mathrm{CN}$ - and $\mathrm{CO}$. 


\subsubsection{Carbon Monoxide}

For carbon monoxide binding, spectra of the native, reduced, and CO-bound of WT$\mathrm{CPO}$ and the mutants were recorded. The ferric enzyme was reduced to ferrous state through the addition of sodium dithionite. The reduced enzyme was then saturated with $\mathrm{CO}$ for a minute for the binding to the heme iron.

\subsubsection{Cyanide}

For cyanide binding, spectra were recorded for WT-CPO and the mutants in $50 \mathrm{mM}$ phosphate buffer at $\mathrm{pH} 5.9$ titrating with increasing concentration, 0-50 mM, of cyanide. The binding affinity was calculated with the following equations [130]:

$$
K_{d}=\frac{[C P O]\left[C N^{-}\right]}{[C P O(C N)]}
$$

where $[\mathrm{CPO}]$ and $\left[\mathrm{CN}^{-}\right]$are the concentration of free $\mathrm{CPO}$ and free cyanide, respectively, and $[\mathrm{CPO}(\mathrm{CN})]$ is the concentration of the complex.

The ratio of the concentration of the complex relative to the total concentration of $\mathrm{CPO}$, $[\mathrm{CPO}]_{0}$ is represented by $\alpha$ :

$$
\alpha=\frac{[C P O(C N)]}{[C P O]_{0}}=\frac{[C P O(C N)]}{[C P O]+[C P O(C N)]}
$$

Combining equation (1) and (2):

$$
\alpha=\frac{\left[C N^{-}\right]}{K_{d}+\left[C N^{-}\right]}
$$

The change in the absorbance intensity of $\mathrm{CPO}(\mathrm{CN})$ complex formation was used as an indication of the formation of the complex. Then equation (2) and (3) can be rewritten as: 


$$
\alpha=\frac{\Delta A_{C P O(C N)}}{\Delta A_{\max }}=\frac{\left[C N^{-}\right]}{K_{d}+\left[C N^{-}\right]}
$$

Assuming $\left[\mathrm{CN}^{-}\right]=\left[\mathrm{CN}^{-}\right]$, equation (4) can be modified as:

$$
\Delta A_{C P O(C N)}=\frac{\Delta A_{\max }\left[C N^{-}\right]_{0}}{K_{d}+\left[C N^{-}\right]_{0}}
$$

Afterward, plotting equation (5) is used to estimate the values of $\Delta \mathrm{A}_{\max }$. Then, $\Delta \mathrm{A}_{\max }$ is used to determine $\mathrm{K}_{\mathrm{d}}$ through equation (4).

\subsubsection{Circular dichroism spectroscopy}

A JASCO spectrometer was used to obtain the CD spectra for $1.3 \mu \mathrm{M}$ WT- and the mutants in $50 \mathrm{mM}$ phosphate $\mathrm{pH}$ 5.90. The spectrum recorded in between the range of 190$300 \mathrm{~nm}$ to monitor the proteins. The CDSSTR method is used to determine the secondary structure's composition. This method performs calculations on the secondary structure composition with a set of reference proteins and those that does not match the characteristic of the sample protein were removed from the reference set.

\subsection{Results}

\subsubsection{UV-Visible spectroscopic properties of WT-, P28A-, P30A-, and P28A/P30A- CPO}

The UV-Vis absorption spectra of WT-, P28A-, P30A-, and P28A/P30A-CPO provided the spectroscopic signature of the enzymes and revealed important information on the heme center. In Figure 2.6, the black line represented the spectrum of WT-CPO and it clearly shows the Soret peak at $398 \mathrm{~nm}$ as well as $\beta$ and $\alpha$ peaks at 515 and $545 \mathrm{~nm}$, respectively. The Soret and $\beta$ peak as observed for WT-CPO has been used to characterize the ferric heme protein to be penta-coordinated as a high-spin species [62]. 
The Soret peak of the CPO mutants is at $420 \pm 1 \mathrm{~nm}$ while $\beta$ and $\alpha$ peaks are located around 540 and $570 \mathrm{~nm}$, respectively (Table 2.3). These shifted peaks suggested the presence of a distal ligand bound to the ferric heme existing in a low-spin hexacoordinate state. Similar spectra have been observed for WT-CPO when the enzyme was cooled to 77 $\mathrm{K}$, implicating that the protein goes through a high-spin to low-spin transition as temperature changes [62]. In addition, the three CPO mutants displayed a clear $\delta$-band at $\sim 362 \mathrm{~nm}$ which provided additional support for the presence of a low-spin heme iron. The peak at $\sim 650 \mathrm{~nm}$ is known as the ligand to metal charge transfer (LMCT) peak and has been proposed to act as a high-spin state marker for heme-thiolate proteins. The intensity of the LMCT peak for the mutants was relatively low as compared to WT-CPO indicating the different spin-state.

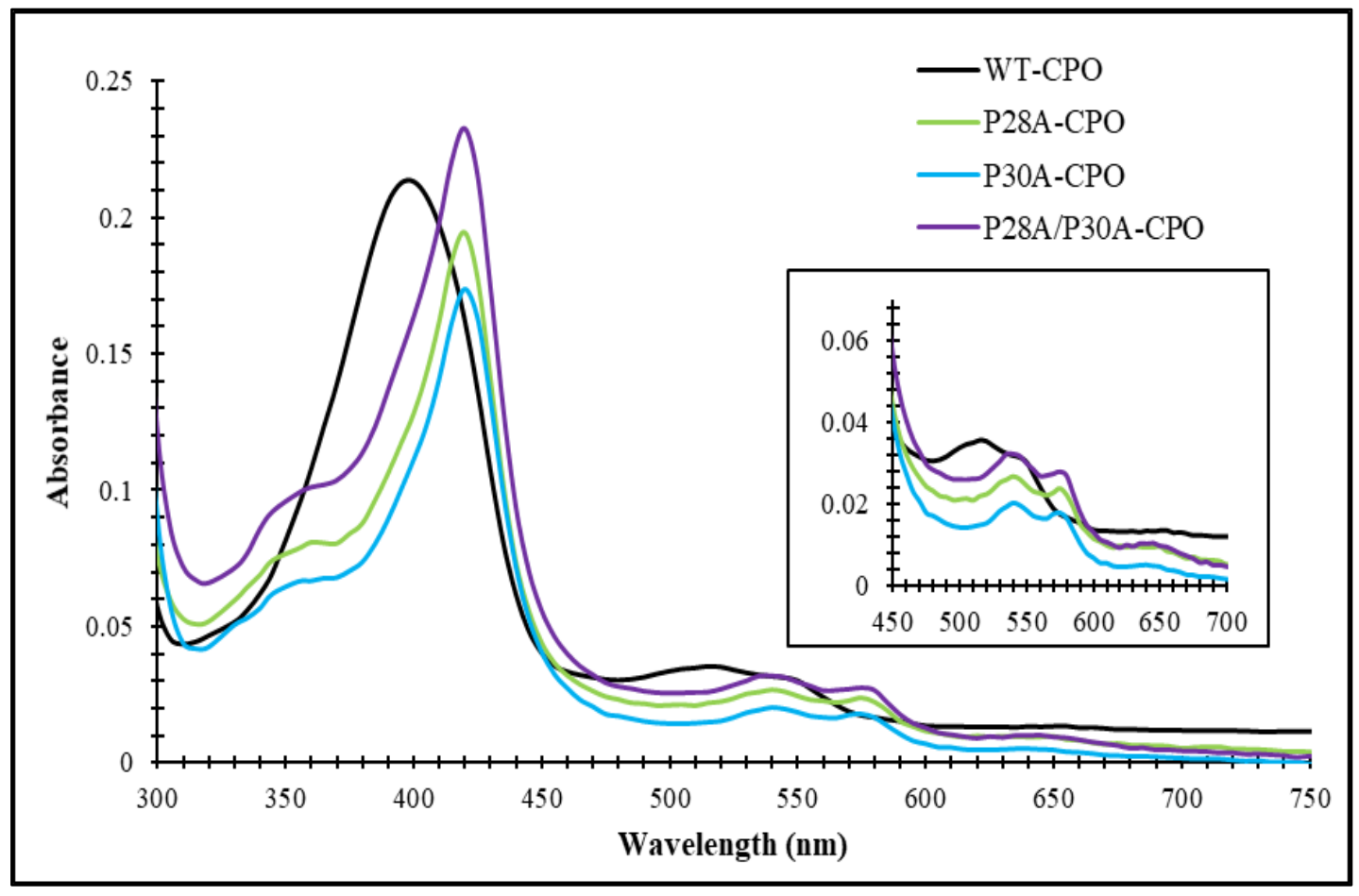

Figure 2.6 UV-Vis Spectra of WT-, P28A-, P30A-, and P28A/P30A-CPO in $50 \mathrm{mM}$ phosphate buffer, pH 5.90. 
Table 2.3 UV-Vis Spectral properties of WT-CPO and the CPO mutants.

\begin{tabular}{|l|r|r|r|r|r|}
\hline Proteins & \multicolumn{1}{|c|}{$\delta$-band } & Soret band & $\beta$-band & $\alpha$-band & CT-band \\
\hline WT-CPO & -- & $398 \mathrm{~nm}$ & $516 \mathrm{~nm}$ & $547 \mathrm{~nm}$ & $649 \mathrm{~nm}$ \\
\hline P28A-CPO & $354 \mathrm{~nm}$ & $420 \mathrm{~nm}$ & $539 \mathrm{~nm}$ & $569 \mathrm{~nm}$ & $650 \mathrm{~nm}$ \\
\hline P30A-CPO & $\sim 362 \mathrm{~nm}$ & $420 \mathrm{~nm}$ & $540 \mathrm{~nm}$ & $570 \mathrm{~nm}$ & $649 \mathrm{~nm}$ \\
\hline P28A/P30A-CPO & $\sim 362 \mathrm{~nm}$ & $420 \mathrm{~nm}$ & $538 \mathrm{~nm}$ & $569 \mathrm{~nm}$ & $651 \mathrm{~nm}$ \\
\hline
\end{tabular}

A possible identity for the distal ligand of the low-spin species could be a water molecule as the spectra of the CPO mutants resembled that of camphor-free P450cam; however, an amino acid in the distal pocket could also be possible. To investigate the properties of the distal ligand as well as the influence of $\mathrm{pH}$ on the structure of the CPO mutants, $\mathrm{pH}$ studies on P28A-, P30A-, and P28A/P30A-CPO were performed in phosphate buffer as shown in the next section.

\subsection{2 pH study on P28A-, P30A-, and P28A/P30A-CPO}

Based on previously reported studies, WT-CPO is stable and the heme iron remains in high-spin state at $\mathrm{pH}$ 2.50-6.00. However, the UV-Vis spectra of the CPO mutants at $\mathrm{pH}$ 2.50-6.00 showed otherwise. The low-spin heme iron of P28A-and P30A-CPO is stable at $\mathrm{pH}$ higher than 3.00. As $\mathrm{pH} 3.00$, the Soret peak $(420 \mathrm{~nm})$ decreased as a peak around 398 $\mathrm{nm}$ began to form (Figure 2.7 and 2.8). This low-spin to high-spin transition is better observed as $\mathrm{pH} 2.50$ where double peaks were observed in the Soret band. The double peaks indicated the presence of mixed state where the distal ligand began to be protonated. This transition revealed the distal ligand has a really acidic $\mathrm{pK}_{\mathrm{a}}$ (below 3.00). The low $\mathrm{pK}_{\mathrm{a}}$ of the distal ligand is quite unusual for a water molecule; however, computational studies 
have suggested that the proximal region of CPO can downregulate the $\mathrm{pK}_{\mathrm{a}}$ of the heme distal ligand. As for the double mutant, P28A/P30A-CPO similar result was observed throughout the tested $\mathrm{pH}$ range (Figure 2.9). Interestingly, this acidic ligand of P28A/P30A-CPO seems to have a lesser competing effect on the cyanide binding relative to the two single mutation mutants as illustrated in section 2.3.4.

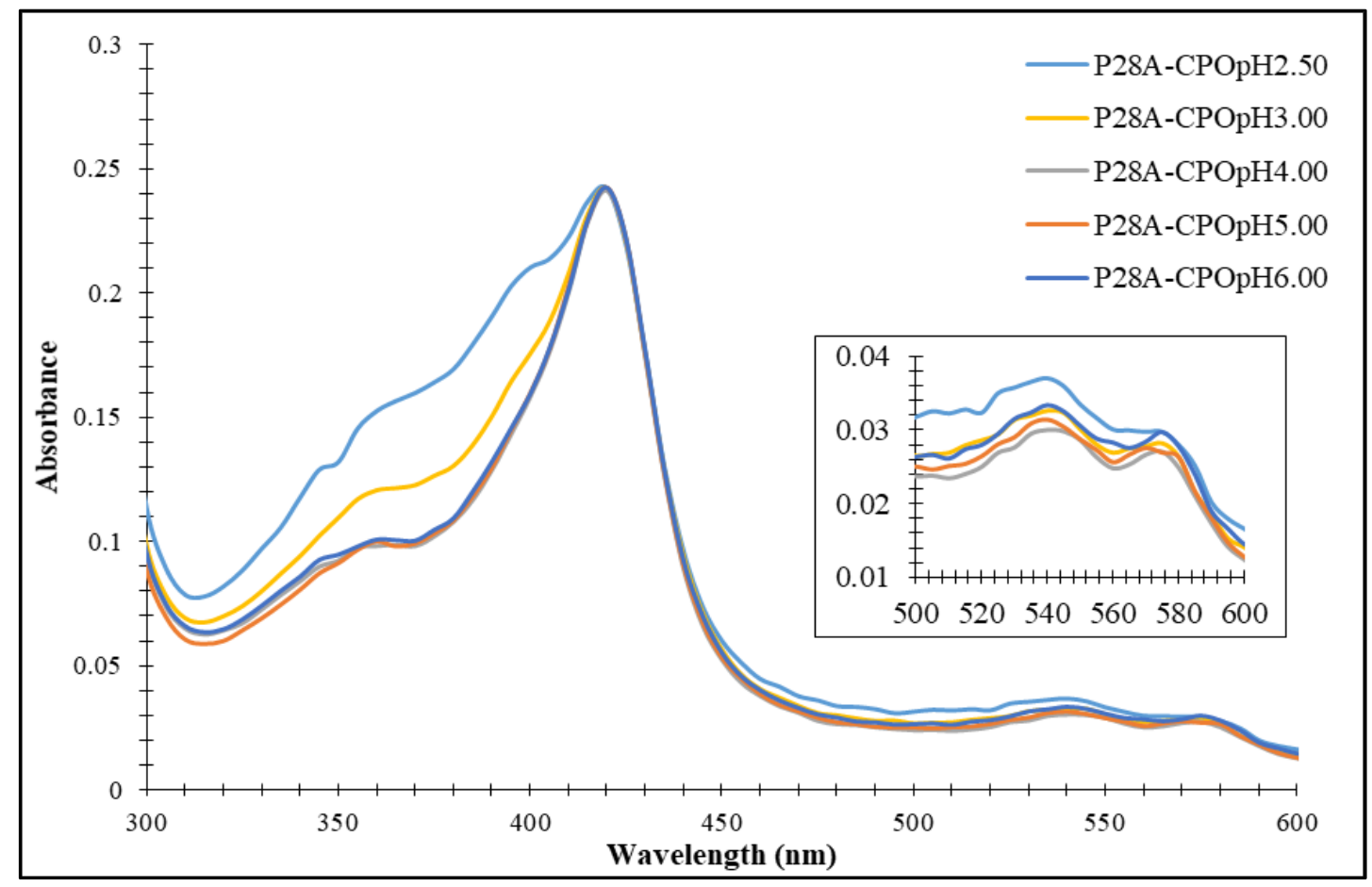

Figure 2.7 UV-Vis Absorption Spectra of P28A-CPO at pH 2.50-6.00. 


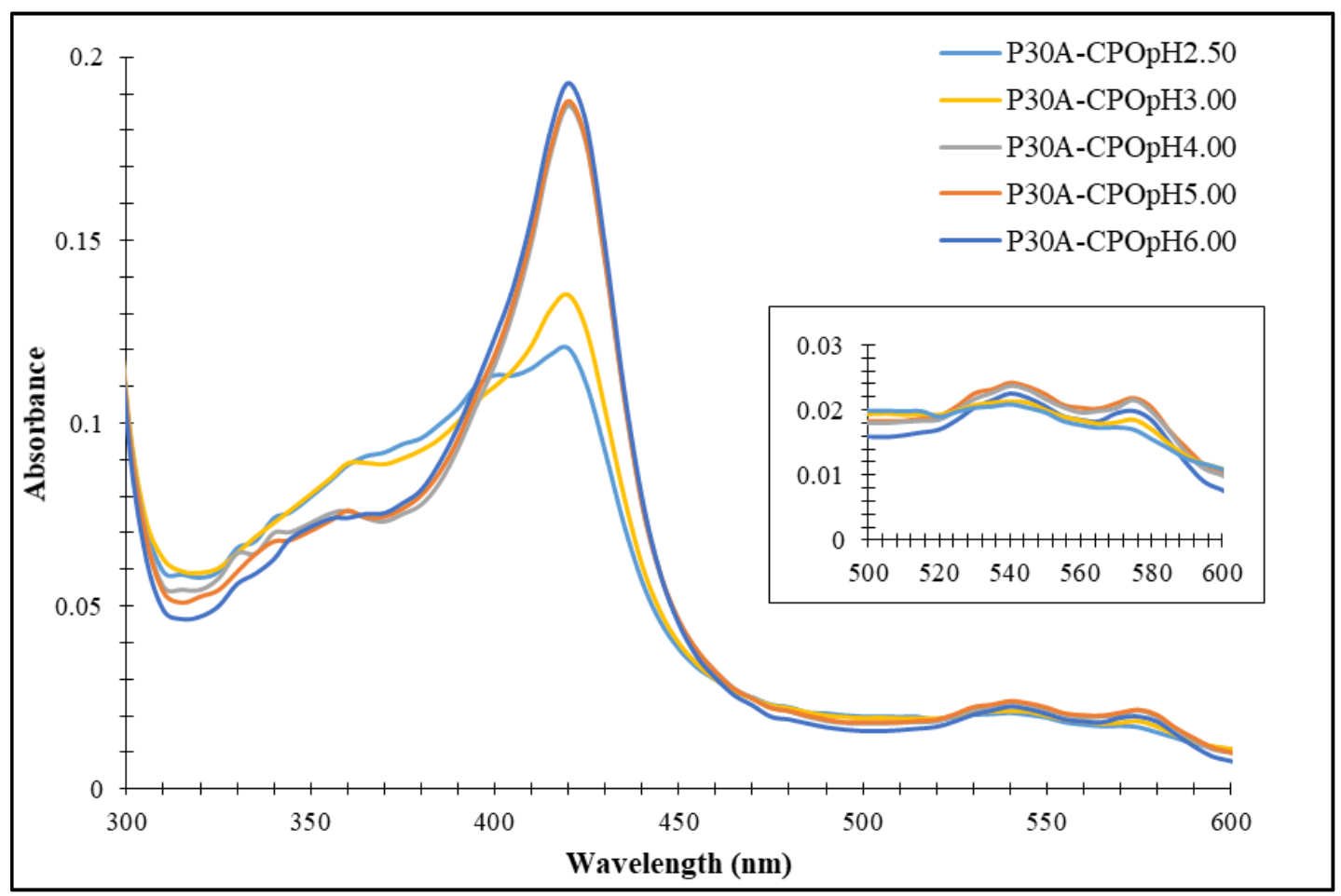

Figure 2.8 UV-Vis Absorption Spectra of P30A-CPO at pH 2.50-6.00.

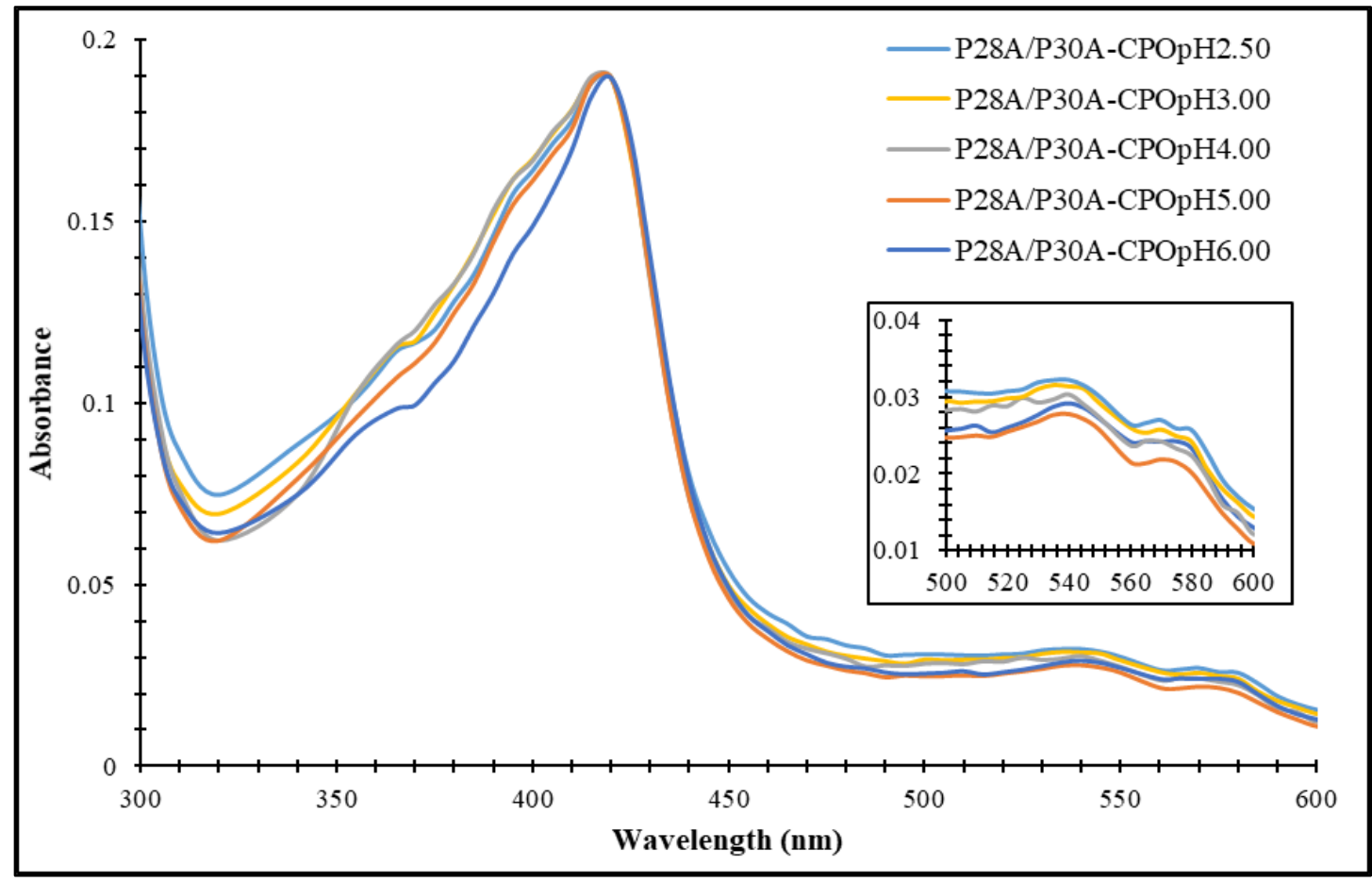

Figure 2.9 UV-Vis Absorption Spectra of P28A/P30A-CPO at pH 2.50-6.00. 


\subsubsection{Carbon Monoxide Binding Study on WT-, P28A-, P30A-, and P28A/P30A- CPO}

The name of P450 originated from the Soret peak of the carbonmonoxy complex at $\sim 450 \mathrm{~nm}$ when carbon monoxide is bound to the enzyme. This characteristic was conserved in heme-thiolate proteins and has been used as an indicator for detecting the presence of a heme thiolate-ligation. The spectra of the carbonmonoxy complex of P28A-, P30A-, and P28A/P30A-CPO were similar to that of WT-CPO (Figure 2.10, 2.11, 2.12, and 2.13 and Table 2.4). Even though the spin state of the ferric WT-CPO and the CPO mutants was different, their Soret band was shifted to $408 \mathrm{~nm}$ after the reduction of the heme iron. The spectra of the P28A-CO, P30A-CO, and P28A/P30A-CO complexes were almost identical to the WT-CPO-CO complex with the Soret peak near $445 \mathrm{~nm}$. Therefore, the mutation(s) did not induce a change on the identity of the proximal ligand in CPO.

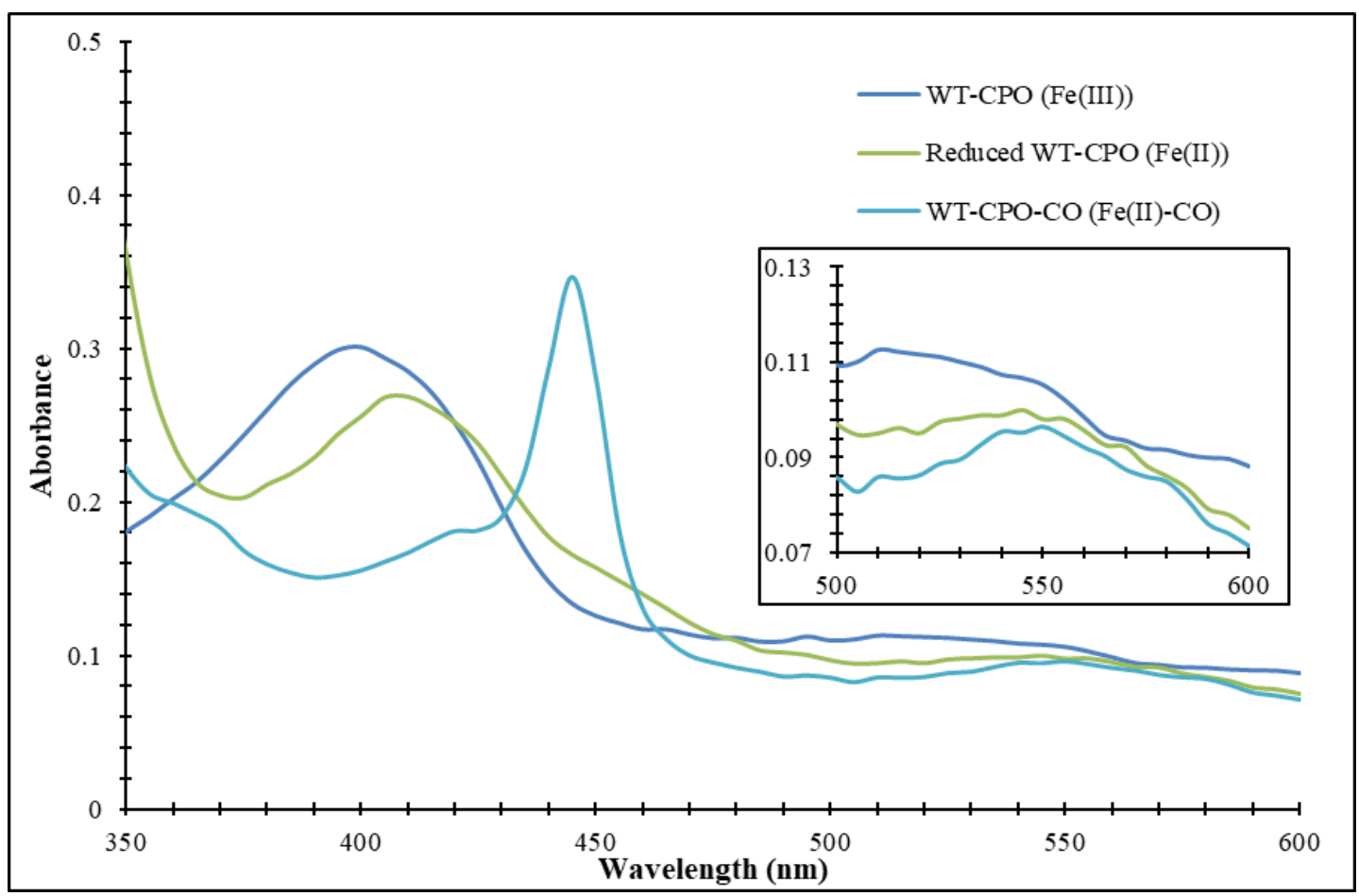

Figure 2.10 UV-Vis Absorption Spectra of ferric, ferrous and CO-bound WT-CPO. 


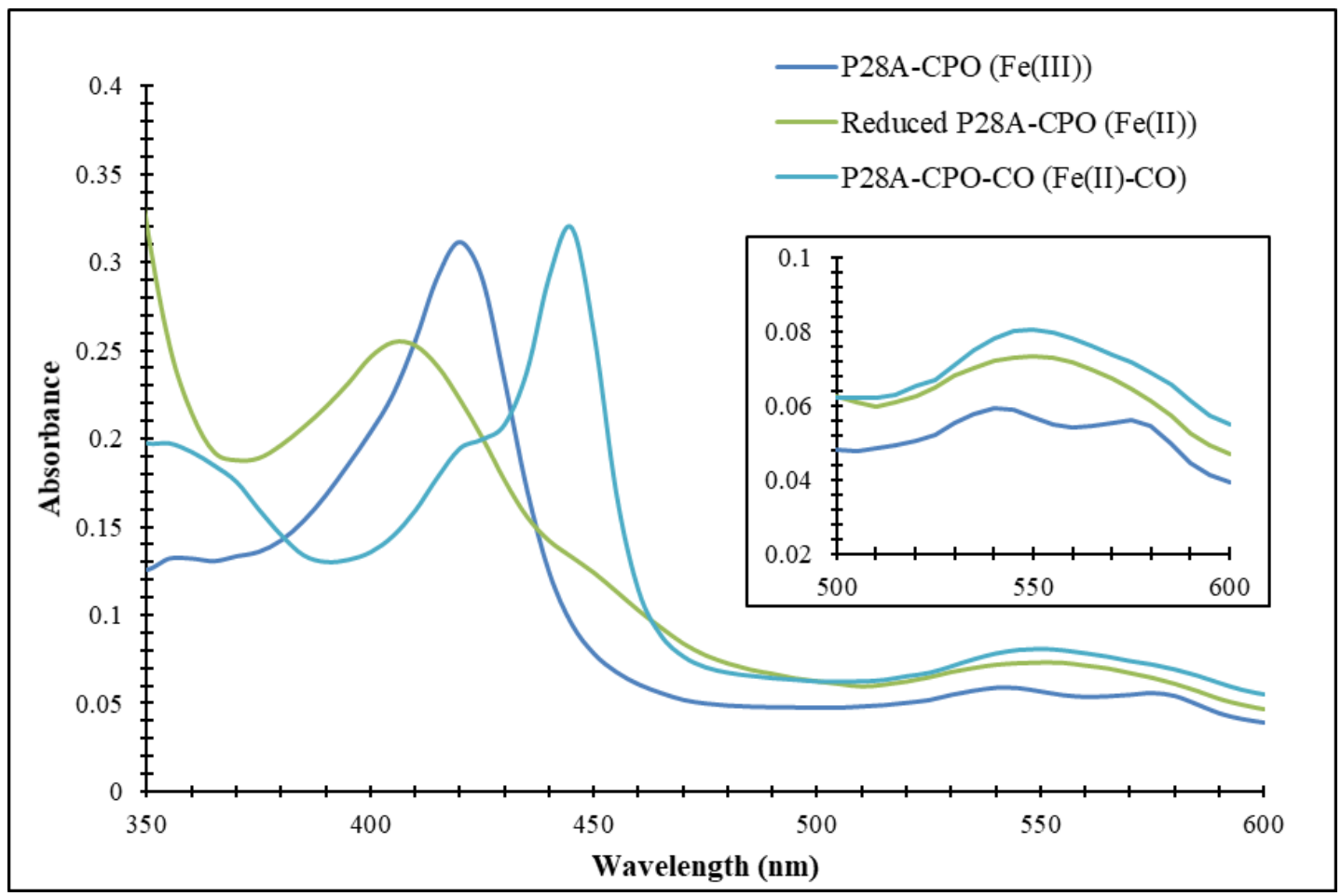

Figure 2.11 UV-Vis Absorption Spectra of ferric, ferrous and CO-bound P28A-CPO.

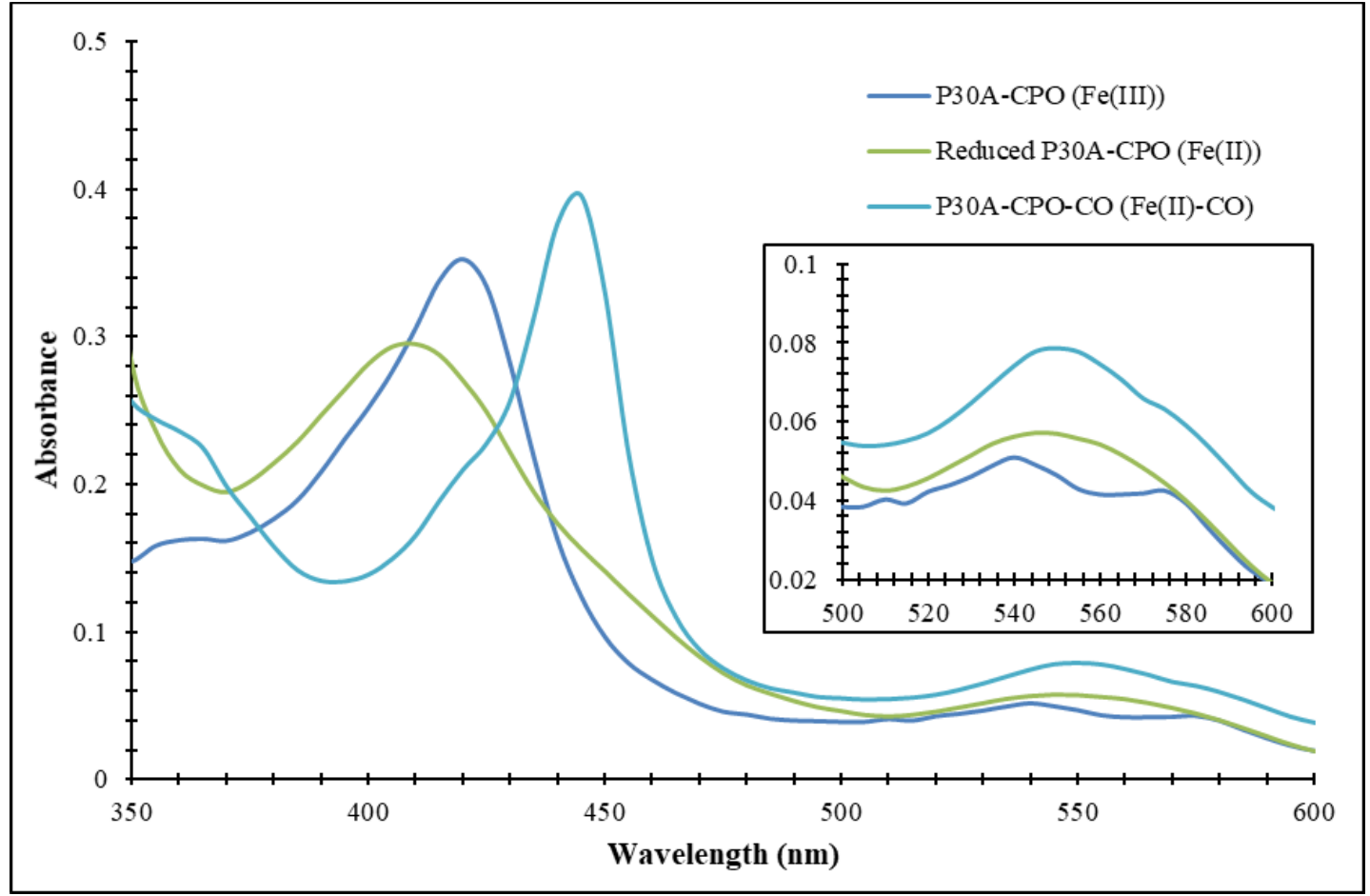

Figure 2.12 UV-Vis Absorption Spectra of ferric, ferrous and CO-bound P30A-CPO. 


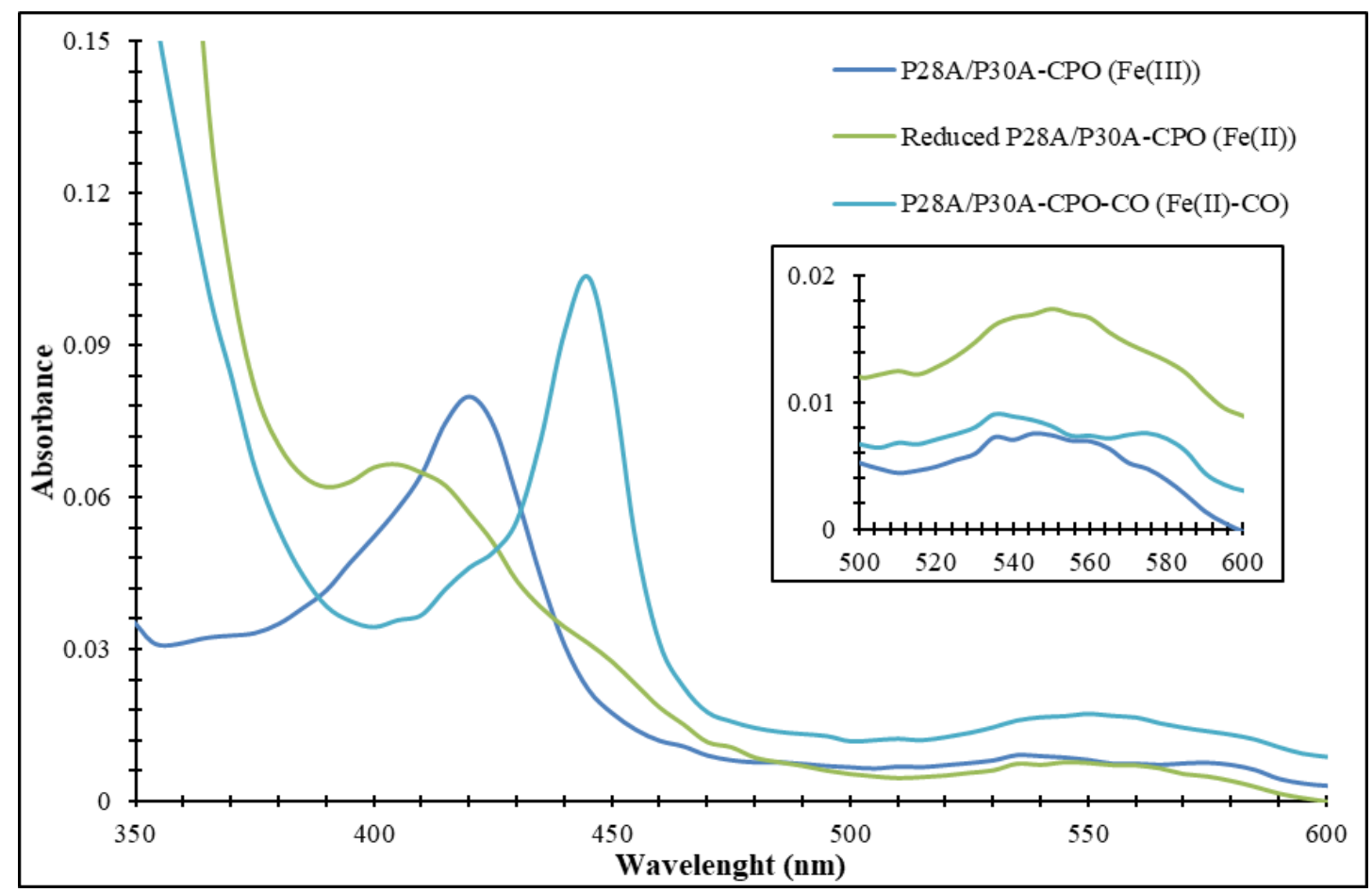

Figure 2.13 UV-Vis Absorption Spectra of ferric, ferrous and CO-bound P28A/P30ACPO.

Table 2.4 UV-Vis spectral properties of ferrous and CO-bound WT-CPO and CPO mutants.

\begin{tabular}{|l|l|l|}
\hline Proteins & Soret band & $\beta$-band \\
\hline Ferrous WT-CPO & $408 \mathrm{~nm}$ & $546 \mathrm{~nm}$ \\
\hline Ferrous-CO complex WT-CPO & $445 \mathrm{~nm}$ & $550 \mathrm{~nm}$ \\
\hline Ferrous P28A-CPO & $406 \mathrm{~nm}$ & $551 \mathrm{~nm}$ \\
\hline Ferrous-CO complex P28A-CPO & $444 \mathrm{~nm}$ & $548 \mathrm{~nm}$ \\
\hline Ferrous P30A-CPO & $408 \mathrm{~nm}$ & $545 \mathrm{~nm}$ \\
\hline Ferrous-CO complex P30A-CPO & $444 \mathrm{~nm}$ & $550 \mathrm{~nm}$ \\
\hline Ferrous P28A/P30A-CPO & $409 \mathrm{~nm}$ & $549 \mathrm{~nm}$ \\
\hline Ferrous-CO complex P28A/P30A-CPO & $444 \mathrm{~nm}$ & $549 \mathrm{~nm}$ \\
\hline
\end{tabular}




\subsubsection{Cyanide Binding Study on WT-, P28A-, P30A-, and P28A/P30A-CPO}

Cyanide is a known ligand that binds to heme proteins. As a weakly acidic ligand, cyanide binds to $\mathrm{CPO}$ as $\mathrm{HCN}$ and then deprotonate to $\mathrm{CN}^{-}$during the ligation to the heme iron. Upon binding to $\mathrm{CPO}$, cyanide converts the high-spin ferric heme iron to a low-spin state. The binding of this exogenous ligand to P28A-, P30A-, and P28A/P30A-CPO were evaluated and compared to that of WT-CPO. The binding of cyanide to WT-CPO was supported by the shift of the Soret peak from $398 \mathrm{~nm}$ to $441 \mathrm{~nm}$ and only one isosbestic point was observed throughout the titration (Figure 2.14). The single isosbestic point at $426 \mathrm{~nm}$ indicates the equilibrium between free and cyanide bound CPO and the calculated dissociation constant $\left(\mathrm{K}_{\mathrm{d}}\right)$ for WT-CPO is $0.068 \mathrm{mM}$.

When $2 \mathrm{mM} \mathrm{CN}$ - is added to P28A-CPO, the Soret peak at $420 \mathrm{~nm}$ began to shift to $434 \mathrm{~nm}$ (Figure 2.15). The absorbance of the new Soret peak increased as the titration procced. During the titration, an isosbestic point at $429 \mathrm{~nm}$ is observed which indicates the change between free and bound states. The dissociation constant $\left(\mathrm{K}_{\mathrm{d}}\right)$ for P28A-CPO was calculated to be $7.4 \mathrm{mM}$ which is significantly greater than WT-CPO. Therefore, cyanide bound to P28A-CPO with a lower affinity than WT-CPO.

Similar spectra were observed for P30A-CPO as seen in Figure 2.16, the Soret peak shifted with the addition of cyanide. The new Soret peak at $439 \mathrm{~nm}$ increased when the concentration of the titrated cyanide increased. However, no clear isosbestic point was observed throughout this titration. The calculated $\mathrm{K}_{\mathrm{d}}$ for P30A-CPO is $4.9 \mathrm{mM}$ which is lower than P28A-CPO but still much higher than WT-CPO. A possible explanation for the decrease in the affinity of cyanide for the mutants relative to WT-CPO is that the mutants 
exist in a low-spin state rather than high-spin. Therefore, cyanide must compete with the existing ligand thus the $\mathrm{K}_{\mathrm{d}}$ increased for the CPO mutants.

Interestingly, the double mutant, P28A/P30A-CPO, has the lowest $\mathrm{K}_{\mathrm{d}}$ of $0.91 \mathrm{mM}$ among the three CPO mutants. The Soret at $420 \mathrm{~nm}$ shifted to $435 \mathrm{~nm}$ as the concentration of $\mathrm{CN}$ - increased as observed in Figure 2.17. Similar to P30A-CPO, no clear isosbestic point is observed for the double mutant. Therefore, mutation on any of the proline residues adjacent to the proximal cysteine will alter the binding affinity of cyanide toward the heme center as summarized in Table 2.5.

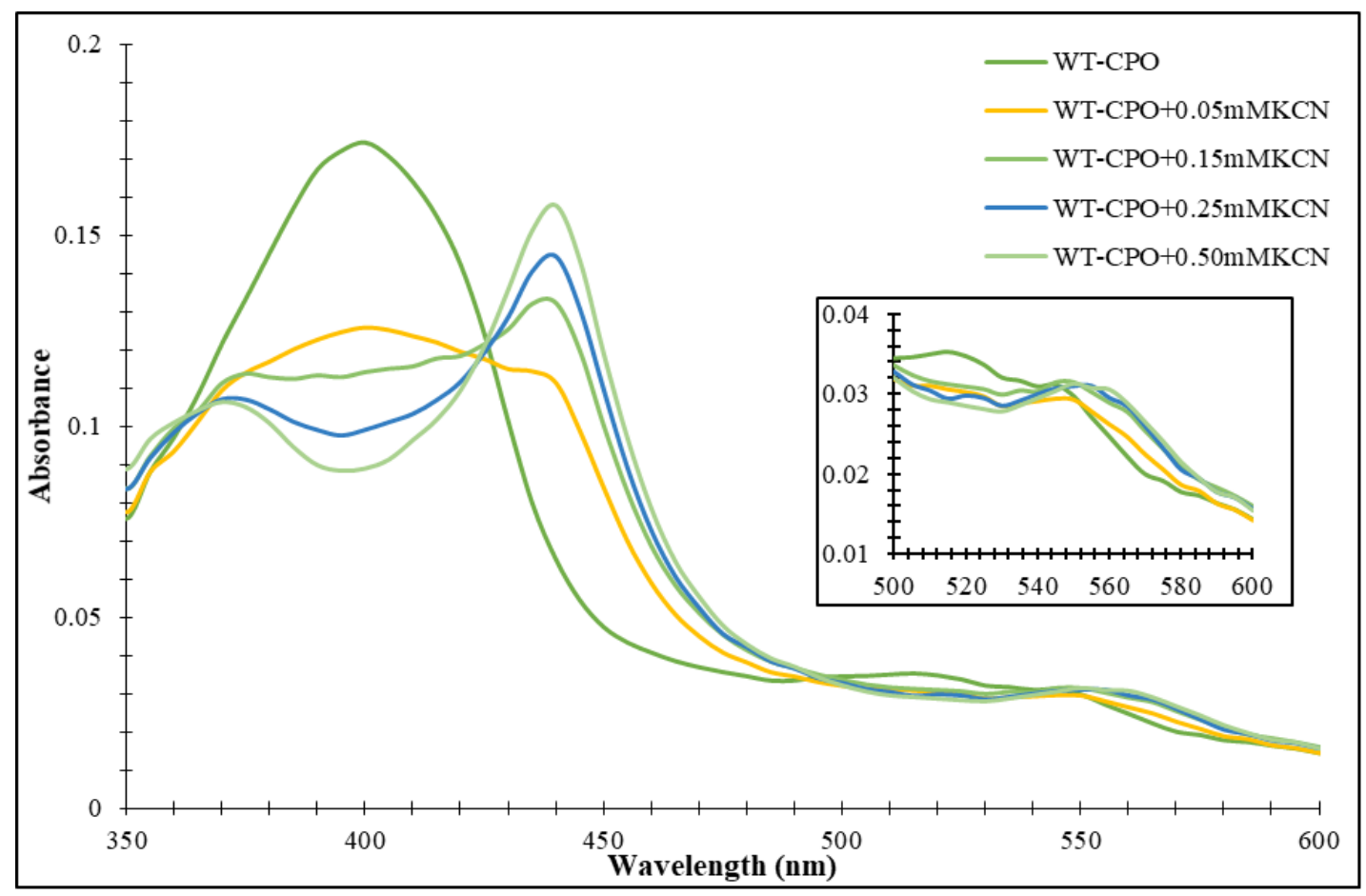

Figure 2.14 Titration of cyanide onto WT-CPO in $25 \mathrm{mM}$ phosphate buffer pH 5.90. 


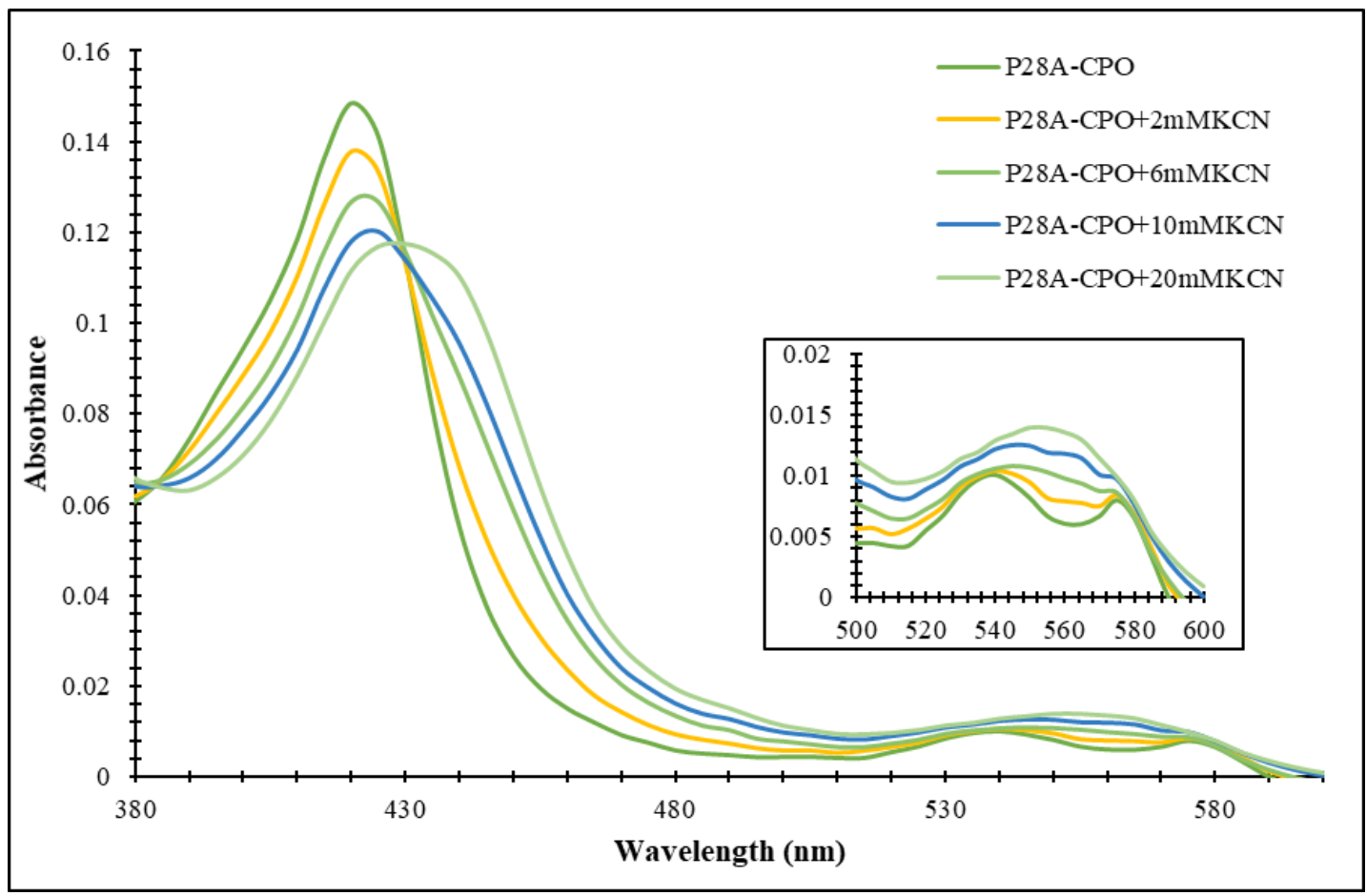

Figure 2.15 Titration of cyanide onto P28A-CPO in $25 \mathrm{mM}$ phosphate buffer pH 5.90.

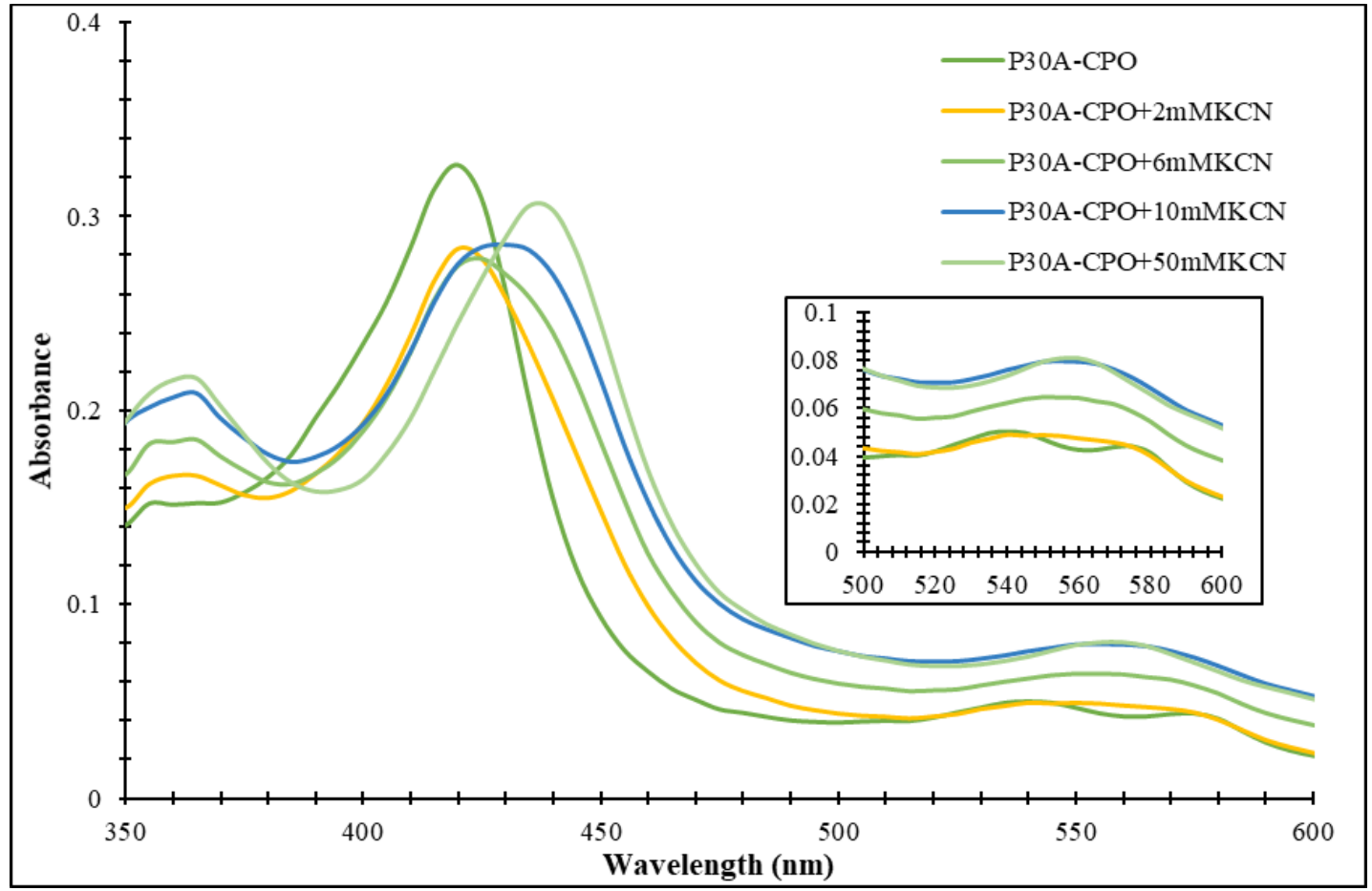

Figure 2.16 Titration of cyanide onto P30A-CPO in $25 \mathrm{mM}$ phosphate buffer pH 5.90. 


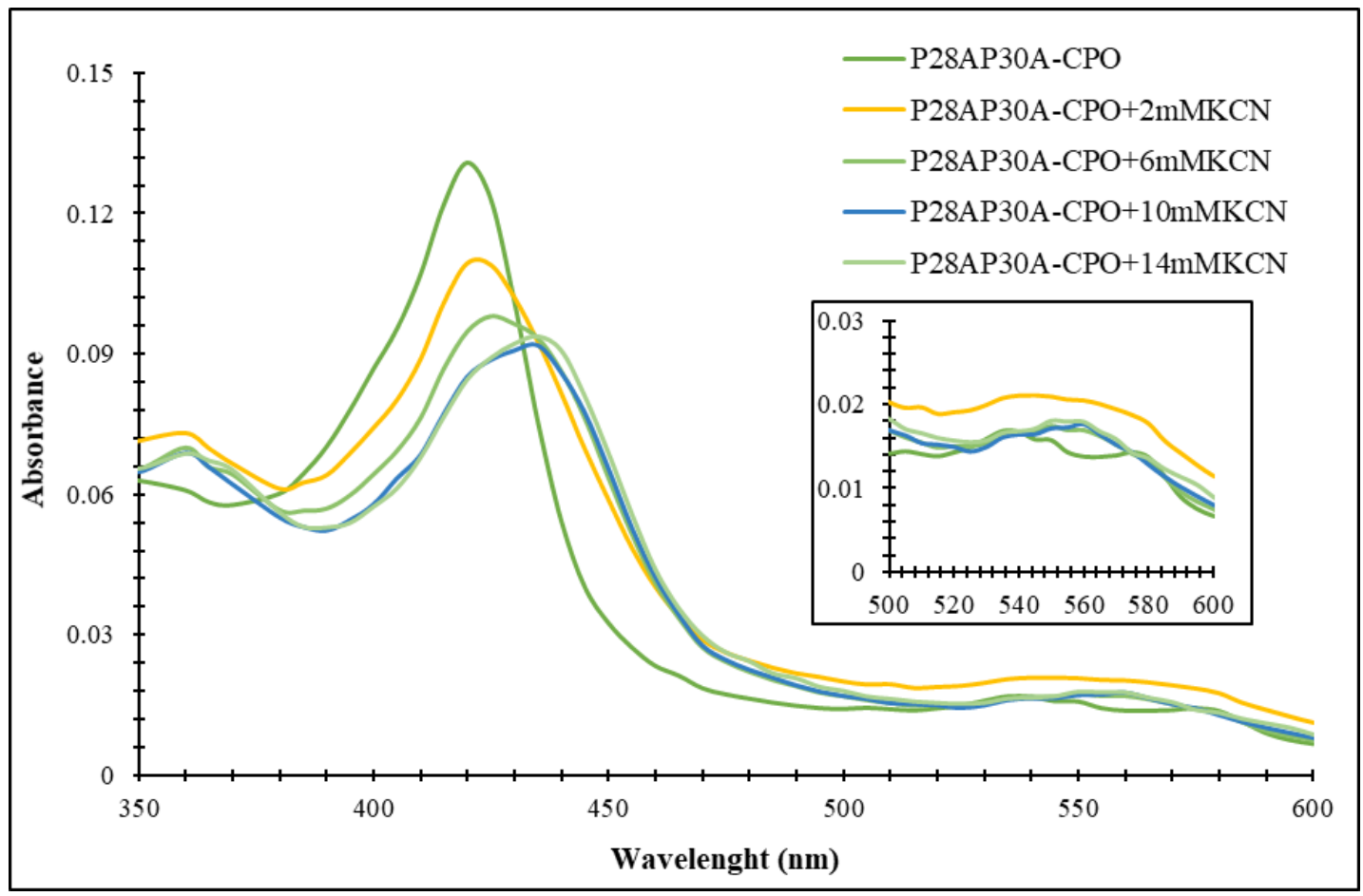

Figure 2.17 Titration of cyanide onto P28A/P30A-CPO in $25 \mathrm{mM}$ phosphate buffer pH 5.90.

Table 2.5 Dissociation Constant of WT-, P28A-, P30A- and P28A/P30A-CPO in 25 $\mathrm{mM}$ phosphate buffer $\mathrm{pH}=\mathbf{5 . 9 0}$.

\begin{tabular}{|l|c|}
\hline Protein & Dissociation Constant \\
\hline WT-CPO & $0.068 \mathrm{mM}$ \\
\hline P28A-CPO & $7.4 \mathrm{mM}$ \\
\hline P30A-CPO & $4.9 \mathrm{mM}$ \\
\hline P28A/P30A-CPO & $0.91 \mathrm{mM}$ \\
\hline
\end{tabular}




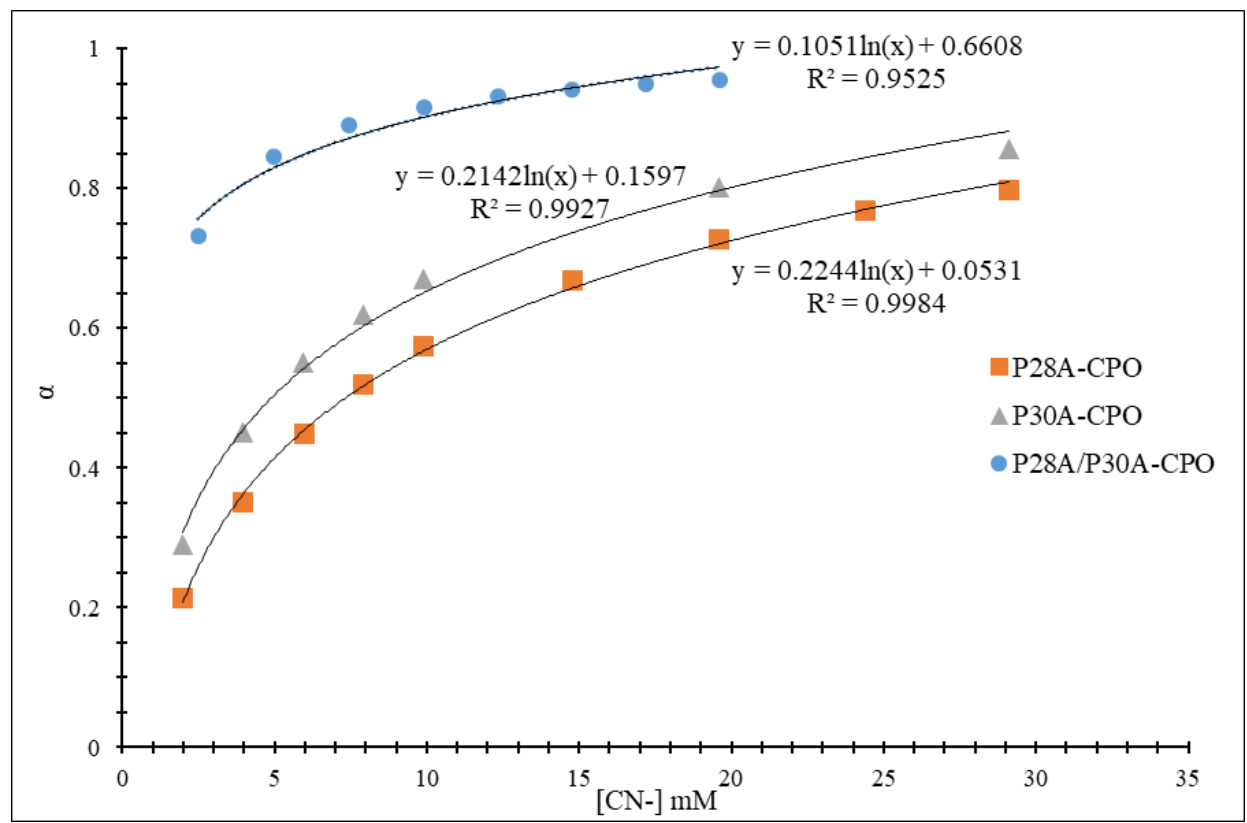

Figure 2.18 $\mathrm{K}_{\mathrm{d}}$ determination for the CPO mutants (WT-CPO is not shown in this figure, but similar method was used to determine the dissociation constant).

\subsubsection{Circular Dichroism Spectroscopy of WT, P28A, P30A, and P28A/P30A CPO}

To further investigate whether the change in the binding affinity is correlated to a structural change induced by the mutation, the secondary structure composition of the CPO mutants was determined through circular dichroism spectroscopy. The CD-spectrum of WT-CPO revealed that the secondary structure of this enzyme was comprised of approximately $34 \%$ and $22 \%$ of alpha-helices and beta-sheet, respectively (Figure 2.19 \& Table 2.6). For P28A- and P30-CPO, the alpha-helices were approximately $31 \%$ and $28 \%$, respectively. The beta-sheets were estimated to be $22 \%$ and $23 \%$ for P28A- and P30ACPO, respectively. These two mutations seem to cause a disruption on the secondary structure of $\mathrm{CPO}$ with less than $6 \%$ difference in alpha-helices and barely any difference in beta-sheets composition. The difference in catalytic activities, as explained in the next chapter, and binding affinity can be caused by the change in the $\alpha$-helix dipole in helix A. 
Therefore, the mutation might induced a change in the tertiary structure of the mutants, such as the orientation of the proximal helix, that could lead to the increase or decrease in redox potential and binding affinity.

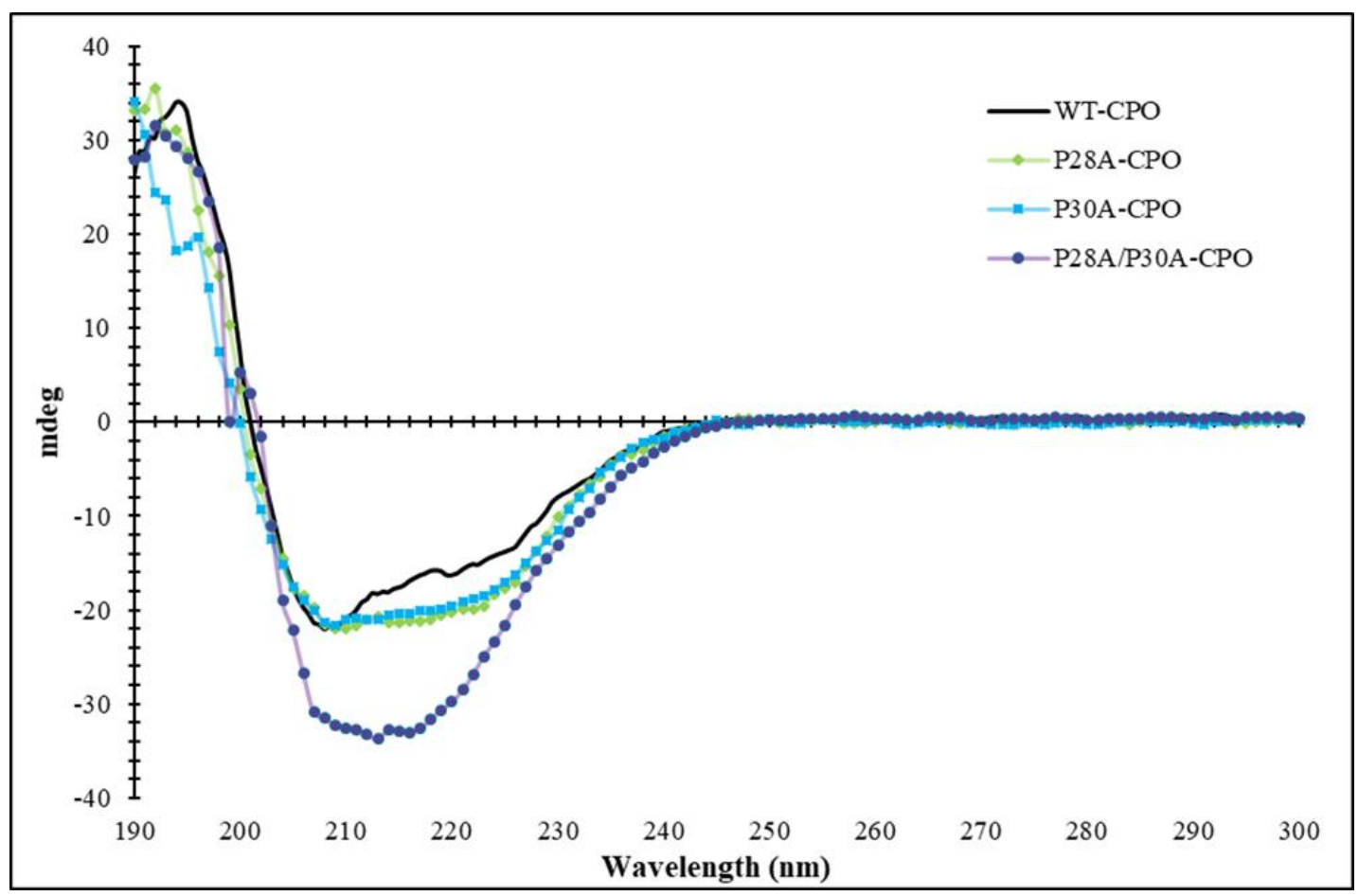

Figure 2.19 Secondary CD Spectra of $1.3 \mu \mathrm{M}$ WT and mutant CPO in $25 \mathrm{mM}$ phosphate buffer, pH 5.90.

Table 2.6 Secondary Structure Composition of WT-, P28A-, P30A- and P28A/P30ACPO in $25 \mathrm{mM}$ phosphate buffer $\mathrm{pH}=5.90$.

\begin{tabular}{|l|l|l|}
\hline Protein & $\alpha$-helix & $\beta$-sheet \\
\hline WT-CPO & $34 \%$ & $22 \%$ \\
\hline P28A-CPO & $31 \%$ & $22 \%$ \\
\hline P30A-CPO & $28 \%$ & $23 \%$ \\
\hline P28A/P30A-CPO & $45 \%$ & $34 \%$ \\
\hline
\end{tabular}

Interestingly, the CD-spectrum of the double mutant, P28A/P30A-CPO, displayed significant difference than that of the other two mutants. The secondary composition of $\mathrm{P} 28 \mathrm{~A} / \mathrm{P} 30 \mathrm{~A}-\mathrm{CPO}$ is $45 \%$ and $34 \%$ for alpha-helices and beta-sheet, respectively. As compared to WT-CPO, there were greater than $10 \%$ increase for both alpha-helices and 
beta-sheet composition for the double mutant. The crystal structure of CPO revealed that these proline residues are adjacent to a different hydrogen bonding networks, as Pro28 is adjacent to the hydrogen bonding network form between Arg26-Asn37 and Ala27-Asn33 while Pro30 is adjacent to the amide-sulfur hydrogen bonding network form between Ala31, Leu32, and Cys29. It is possible the mutation on Pro28 or Pro30 would disturb only the adjacent hydrogen bonding network while the double mutants would distort both hydrogen bonding networks thus result in a significant change in the secondary structure of CPO.

\subsection{Conclusion}

The Pro-Cys-Pro tripeptide stretch within the proximal region of CPO is found to be conserved in other heme-thiolate proteins such as UPOs. The proximal region of heme enzymes have been proposed to play an important role in modulating their catalytic reactivity as well as maintaining their structural properties. In the present study, we mutated the proline residues, Pro28 and Pro30, adjacent to the proximal cysteine of CPO to an alanine to examine their effects on the enzyme's structural properties. Three mutants, P28A-, P30A-, and P28A/P30A-CPO, were designed and characterized with spectroscopic techniques.

My results revealed that the CPO mutants at ferric state exist as a low-spin hexacoordinated species with the Soret peak at $420 \mathrm{~nm}$ while WT-CPO, Soret peak at $398 \mathrm{~nm}$, is at a high-spin penta-coordinated state. The low-spin state of the three mutants were further supported by the presence of the $\delta$-band at $354 \mathrm{~nm}, 362 \mathrm{~nm}$, and $326 \mathrm{~nm}$ for P28A, P30A-, and P28A/P30A-CPO, respectively. Further investigation on the properties of the sixth-coordinated ligand of the three mutants revealed the displacement of the distal ligand 
occur at $\mathrm{pH}$ less than 3.00 as supported by the double peaks, which indicated the presence of mix state. The UV-Vis spectra of the CPO-mutants showed resemblance to the resting state of substrate-free P450cam, suggesting the identity of the distal ligand can possibly be a water molecule.

Ligand binding study revealed that the presence of a distal ligand for the CPO mutants influences the binding affinity of cyanide to the heme iron. The calculated $\mathrm{K}_{\mathrm{d}}$ of $\mathrm{CN}$ - for P28A/P30A-CPO is significantly lower than that of P28A- and P30A-CPO. However, the readily high-spin state of WT-CPO allows $\mathrm{CN}$ - to bind more tightly to the heme center relative to the CPO mutants. Therefore, Pro28 and Pro30 are greatly important in maintaining the coordination state of the heme center and mutation of these residues will dramatically changes the binding affinity of exogenous ligand to the ferric heme iron.

Carbon monoxide only bind to the ferrous heme iron to form the carbonmonoxy complex. The binding of carbon monoxide has been employed to probe the proximal ligand of heme proteins as they exert different Soret band. The signature Soret maximum at $~ 445$ $\mathrm{nm}$ for the carbonmonoxy complexes of the $\mathrm{CPO}$ mutants confirmed the presence of a thiolate-ligation. CD spectra of WT-CPO and the $\mathrm{CPO}$ mutants revealed that mutation on Pro28 or Pro30 have only a minimal effect on the enzyme's secondary structure composition. However, P28A/P30A-CPO displayed a significantly change in the CD spectrum. This significant change could be the result of the distortion of the proximal hydrogen bonding networks, the amide-sulfur hydrogen bonding network and the proximal loop hydrogen bonding network between Arg26-Asn37 and Ala27-Asn33. As these hydrogen bonding networks are adjacent to Pro28 and Pro30, mutations on the proline residues can disturb the networks which would lead to a significant change in the secondary 
structure. Therefore, my work indicates that Pro28 and Pro30 have a significant impact on the maintaining the structural properties of $\mathrm{CPO}$ as mutation of these proline residues can induce a change in the proximal helix affecting its interaction with the protein matrix as well as disturbing the adjacent hydrogen bonding networks. Such changes in the structural properties would most likely to influence the biochemical properties of $\mathrm{CPO}$ as they will be discussed in the next chapter. 


\section{CHAPTER III.}

\section{INFLUENCE OF THE PRO-CYS-PRO TRIPEPTIDE IN THE PROXIMAL}

\section{LOOP ON THE CATALYTIC PROPERTIES OF CHLOROPEROXIDASE}

\subsection{Background}

CPO has been known as the most versatile heme enzymes for the past decades and this versatility is linked to the "P450-peroxidases" hybrid structure. Many other classical heme enzymes are mono-functional which mean they can only catalyze a particular type of reaction such as mono-functional catalase. However, CPO can catalyze four different types of reactions and they are peroxidation (peroxidase-like), dismutation (catalase-like), oxygen-insertion (peroxygenase/monooxygenase-like), and halogenation (halogenaselike) reactions. Compound $\mathrm{I}$ is the reactive species responsible for catalyzing the different type of reactions depending on the condition of the system.

The formation of Compound I depends on the presence of an acid-base catalyst, which serves as the "pull effect," and the electron-donating ability of the proximal ligand, that acts as the "push effect" [131]. This "push-pull" effect is crucial for the formation of the Compound I. In CPO, the "push effect" of Cys29 is regulated by the proximal helix such as through the amide-sulfur hydrogen bonding network described in the previous chapter. These hydrogen bonds reduced the electronegativity of the thiolate leading to the increase on the heme iron redox potential as well as reducing the $\mathrm{pK}_{\mathrm{a}}$ of the distal ligand [11, 6369]. Pro28 and Pro30 are located adjacent to the proximal cysteine as well as the amidesulfur hydrogen bonding network. They should play a role in regulating the reactivity of the enzyme through modulating cooperating effect between the $\alpha$-helical conformation of helix A and the amide-sulfur hydrogen bonds. 


\subsubsection{Aim of this study}

In this part of my study, I investigated the effect of substituting Pro28 and Pro30 with Ala in term of CPO's catalytic properties. Three CPO mutants, P28A-, P30A- and P28A/P30A-CPO, were characterized through different assays targeting a specific activity of $\mathrm{CPO}$. The biochemical characterization of the $\mathrm{CPO}$ mutant provided us insight on the importance of these proline residues on CPO-catalyzed one-electron (peroxidation of ABTS) and two-electron oxidation reactions (dismutation of hydrogen peroxide, epoxidation of styrene and halogenation of MCD). My results revealed that Pro28 and Pro30 play a significant role in modulating the versatility of $\mathrm{CPO}$ where their substitution induced significant changes on CPO's catalytic activities.

\subsection{Materials and Methods}

\subsubsection{Materials}

All materials used are the same as those described in section 2.2.1.

\subsubsection{Construction of P28A-, P30A- and P28A/P30A-CPO genes}

P28A-, P30A-, and P28A/P30A-CPO were constructed with procedure similar as those described in section 2.2.2. As for primers, they are listed in table 2.2 for the three CPO mutants.

\subsubsection{Transformation, Expression and Purification of P28A-, P30A- and P28A/P30A-CPO plasmid in Aspergillus niger}

The transformation process is similar as described in section 2.2.3. As for the expression and purification process, the procedures were similar to that described in section 2.2.4. 


\subsubsection{Enzymatic activity assays studies}

\subsubsection{ABTS Assay}

Peroxidase activity of $\mathrm{CPO}$ was measured through the oxidation of 2, 2'-Azinobis-3ethylbenzothiazoline-6-sulfonic acid (ABTS) (Figure 3.1). The reaction system is composed of $\mathrm{CPO}$ in $100 \mathrm{mM}$ phosphate-citrate buffer (pH range 2.50-6.00) containing $38.9 \mathrm{uM}$ ABTS. The reaction was initiated by the addition of $3.6 \mathrm{mM} \mathrm{H}_{2} \mathrm{O}_{2}$ and the rate of peroxidation was monitored at $405 \mathrm{~nm}$.

\subsubsection{MCD Assay}

MCD assay is commonly used to determine the chlorinating activity of haloperoxidase. This assay monitors the chlorination of monochlorodimedone (MCD) to dichlorodimedone (Figure 3.2). The reaction system is composed of CPO in $100 \mathrm{mM}$ potassium-phosphate buffer containing $20 \mathrm{mM} \mathrm{KCl}$ and $0.17 \mathrm{mM} \mathrm{MCD}$ at $\mathrm{pH} 2.50-5.00$. The reaction was initiated by the addition of $2.2 \mathrm{mM} \mathrm{H}_{2} \mathrm{O}_{2}$ while monitoring the rate of chlorination at 278 nm.

\subsubsection{Catalase Assay}

Catalase catalyzes the decomposition of hydrogen peroxide. CPO's catalatic activity is measured by monitoring the oxidative decomposition of hydrogen peroxide to water and dioxygen. The reaction system is composed of $0.05 \%$ hydrogen peroxide in $50 \mathrm{mM}$ phosphate-citrate buffer at $\mathrm{pH}$ 2.50-6.00. The reaction was initiated by the addition of CPO and rate of decomposition was monitored at $240 \mathrm{~nm}$. 

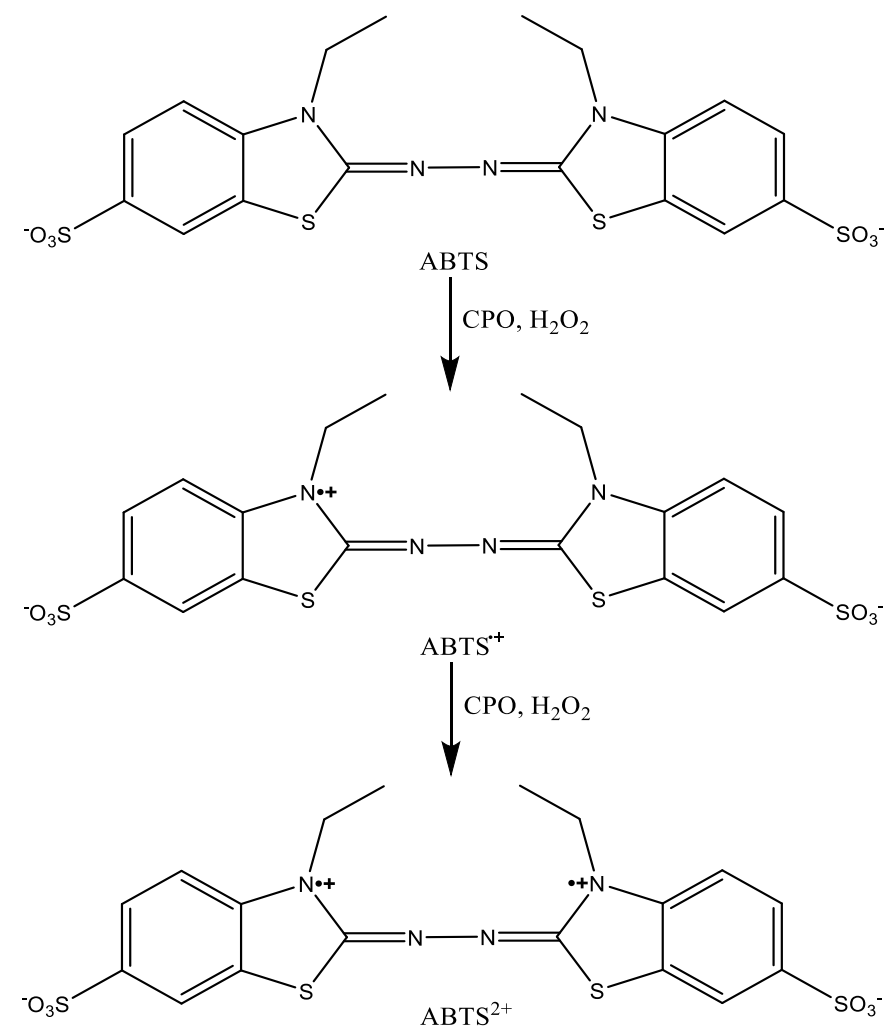

Figure 3.1 CPO-catalyzed oxidation of ABTS
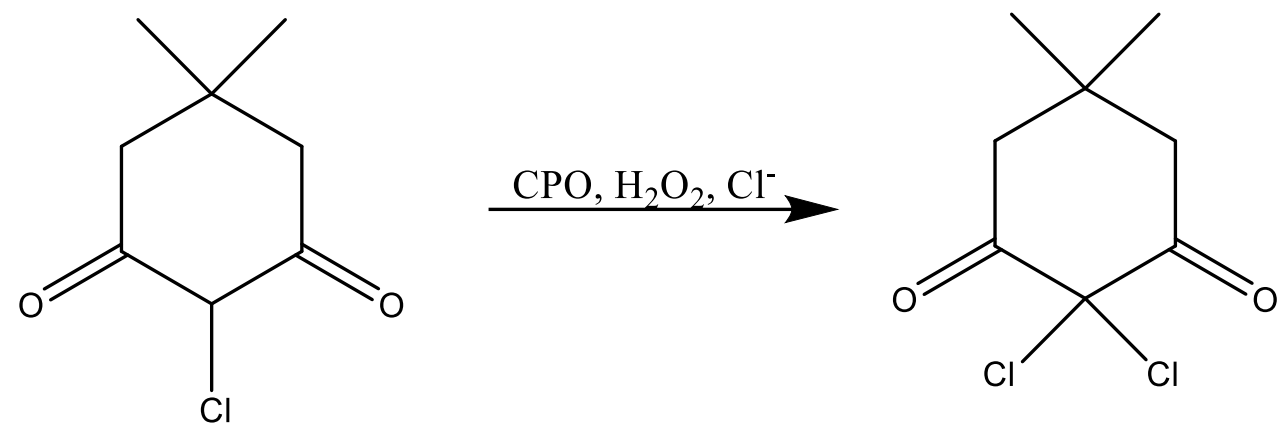

Figure 3.2 CPO-catalyzed chlorination of MCD.

\subsubsection{Epoxidation Assay}

The epoxidation assay measures the epoxidation of styrene catalyzed by CPO (Figure 3.3). The reaction system is composed of CPO in $100 \mathrm{mM}$ sodium-acetate buffer at $\mathrm{pH}$ 2.50-6.00, containing $0.3 \mathrm{mM}$ styrene and initiated by the addition of $0.01 \% \mathrm{H}_{2} \mathrm{O}_{2}$ while monitoring the absorbance at $262 \mathrm{~nm}$. 


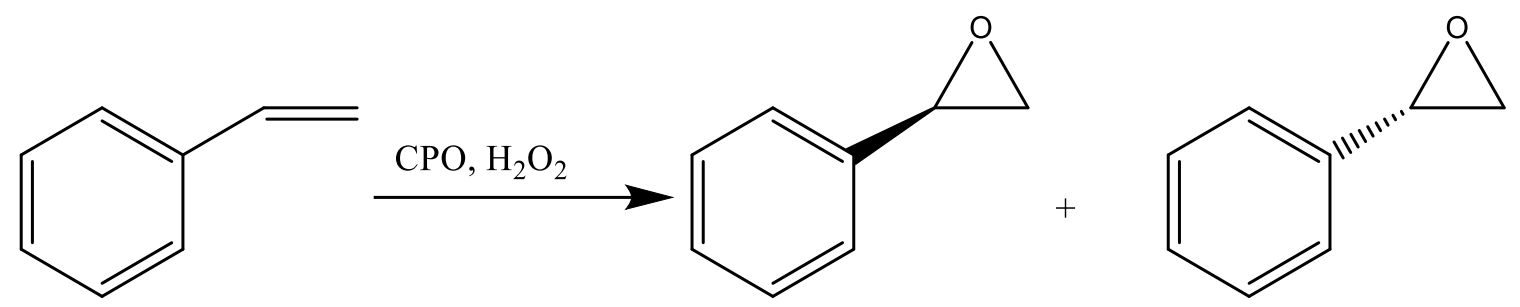

Figure 3.3 CPO-catalyzed epoxidation of styrene yielding $R$-styrene oxide and $S$ styrene oxide.

\subsection{Results}

\subsection{1 pH Profile of the Catalytic Properties of WT-, P28A-, P30A-, and P28A/P30A-CPO}

To investigate the influence of the proximal Pro-Cys-Pro tripeptide stretch on the biochemical properties of CPO, four assays were used to characterize the difference between the WT and mutants. They are peroxidation of ABTS, chlorination of MCD, dismutation of $\mathrm{H}_{2} \mathrm{O}_{2}$, and epoxidation of styrene. These four assays were carried out throughout different $\mathrm{pH}$ condition examining the effects of the residue substitution on the catalytic activities of CPO. The specific activity for each assay were calculated with the rate of the reaction. The rate of the reaction along with the Beer's law was then used to calculates the change in the amount of the substrate or product over time (second). By dividing the amount of enzyme used for the reaction, the specific activity was calculated. The extinction coefficients used for specific activity's calculation for the four assays were listed in Table 3.1. 
Table 3.1 Extinction coefficient of the Four Assays (ABTS, Epoxidation, Dismutation, and Chlorination).

\begin{tabular}{|l|l|l|}
\hline Assays & Wavelength & Extinction Coefficient $\left(\mu \mathrm{M}^{-1} \mathrm{~cm}^{-1}\right)$ \\
\hline ABTS & $405 \mathrm{~nm}$ & 0.0368 \\
\hline Styrene & $262 \mathrm{~nm}$ & $1.08 \times 10^{-3}$ \\
\hline Hydrogen peroxide & $240 \mathrm{~nm}$ & $4.36 \times 10^{-5}$ \\
\hline Monochlorodimedone & $278 \mathrm{~nm}$ & 0.0122 \\
\hline
\end{tabular}

\subsubsection{Peroxidation of ABTS}

ABTS assay is commonly used to measure peroxidation activity of heme peroxidase. When ABTS is oxidized to $\mathrm{ABTS}^{2+}$, a color transition from colorless to a visible green color is observed which this change is monitored at $405 \mathrm{~nm}$ as seen in Figure 3.4. In WT$\mathrm{CPO}$, the optimal $\mathrm{pH}$ for ABTS peroxidation is at $\mathrm{pH} 2.50$. The specific activity decreased significantly at $\mathrm{pH} 3.00$ and is negligible at $\mathrm{pH} 4.00$ or greater. As for the mutants, the peroxidase's activity remained active throughout $\mathrm{pH}$ 2.50-5.00 with the optimal $\mathrm{pH}$ at 3.00. Among the three mutants, P30A-CPO has the highest activity throughout the tested $\mathrm{pH}$ range.

As stated in the introduction, the catalytic cycle of CPO contains intermediates that are responsible for catalyzing certain reaction. In the peroxidation pathway, Compound II is responsible for catalyzing the second one-electron oxidation of the substrate. The peroxidase's activity of the three $\mathrm{CPO}$ mutants is active at $\mathrm{pH}$ greater than 4.00 , suggesting the mutation within the Pro-Cys-Pro tripeptide stretch have a significant influence on the formation of Compound I as well as Compound II. 


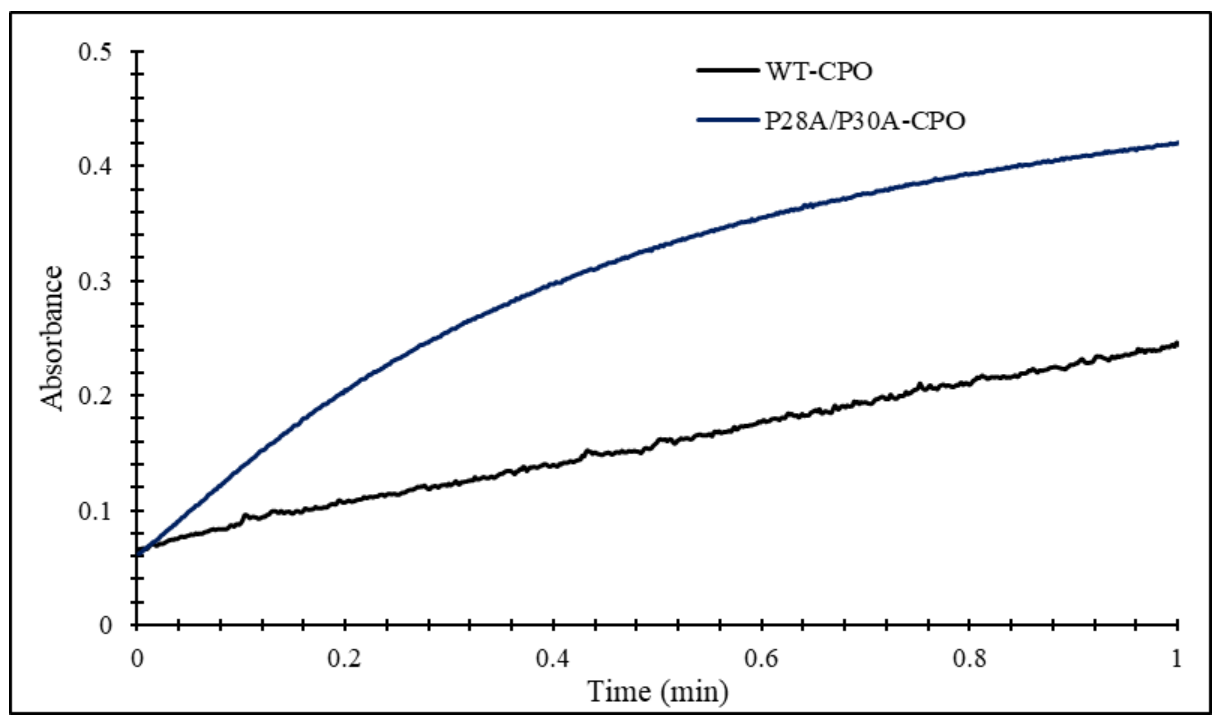

Figure 3.4 Peroxidation of ABTS catalyzed by WT- and P28A/P30A-CPO monitor at 405nm.

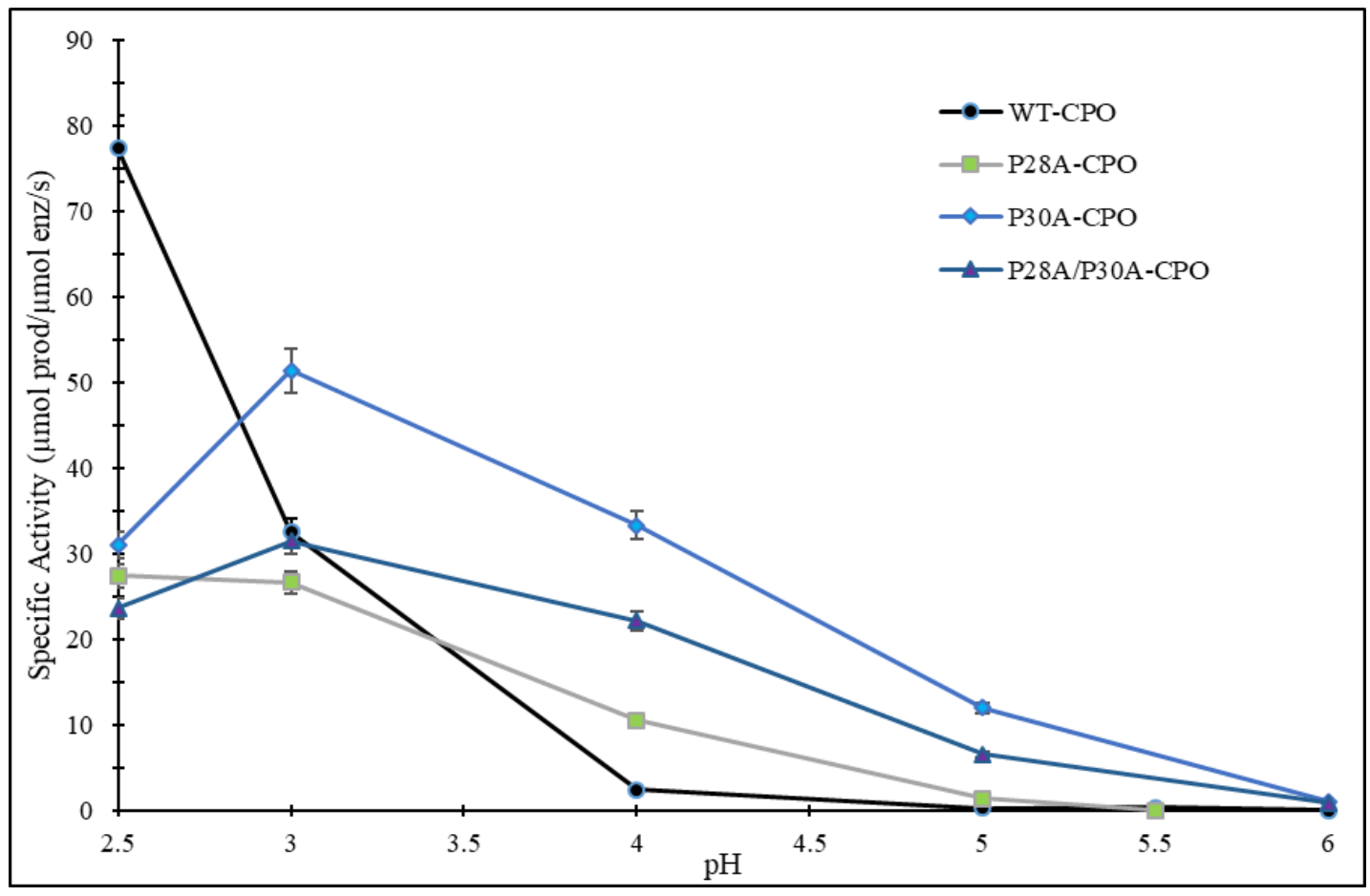

Figure 3.5 Specific activity of WT-, P28A-, P30A- and P28A/P30A-CPO for ABTS assay at pH 2.50-6.00. (Some error bars are not visible on the graph)

As proposed in previous studies, the proximal pocket of $\mathrm{CPO}$ regulates the $\mathrm{pK}_{\mathrm{a}}$ of the distal heme ligand during the formation of Compound I through the interaction of the 
proximal ligand [67]. As in the finding of Dr. Raven's group, the nature of the ferryl species of heme proteins is an unprotonated iron-oxo and protonated iron-oxo for Compound I and Compound II, respectively [132]. Therefore, the regulation of the distal ligand $\mathrm{pK}_{\mathrm{a}}$ caused by the mutation favored the formation of the active protonated Compound II species relative to WT-CPO where significant activities were observed at $\mathrm{pH} \geq 4.00$.

\subsubsection{Epoxidation of Styrene}

WT-CPO catalyzes epoxidation reactions in a stereoselective manner and has been a great interest in pharmaceutical and chemical fields as a potential biocatalyst candidate. The activity of $\mathrm{CPO}$ in the oxygen-insertion pathway is measured through the epoxidation assay by monitoring the decreased in absorbance at $262 \mathrm{~nm}$ (Figure 3.6). The specific activity of WT-CPO for the epoxidation reaction does not have a significant change throughout the tested $\mathrm{pH}$ range which suggested this type of reaction is $\mathrm{pH}$-independent. However, this does not seem to be the case for the CPO mutants. The specific activity of P28A- and P30A-CPO increased significantly as the $\mathrm{pH}$ rise (Figure 3.7). This trend suggested the mutation on Pro28 caused a greater influence on the epoxidation activity of CPO relative to Pro30 mutation. As observed, the double mutant, P28A/P30A-CPO, displays similar epoxidation activity trend along the $\mathrm{pH}$ range as to the other two mutants. 


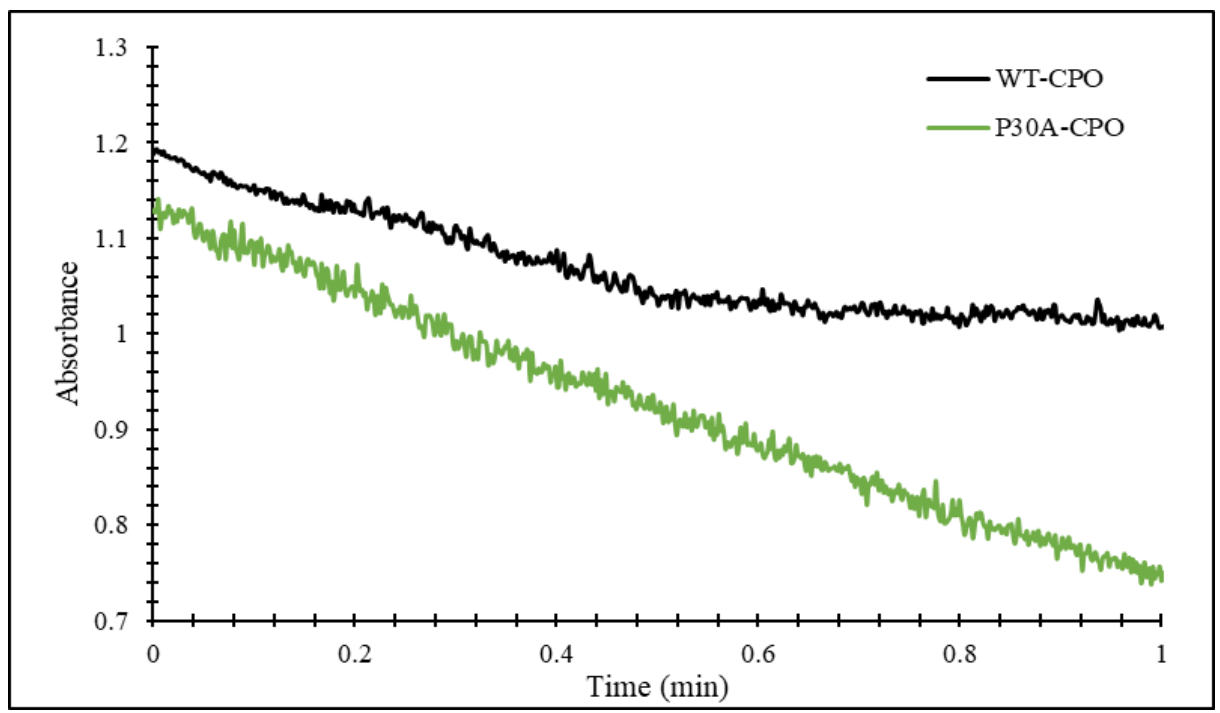

Figure 3.6 Epoxidation of Styrene catalyzed by WT- and P30A-CPO monitor at $262 \mathrm{~nm}$.

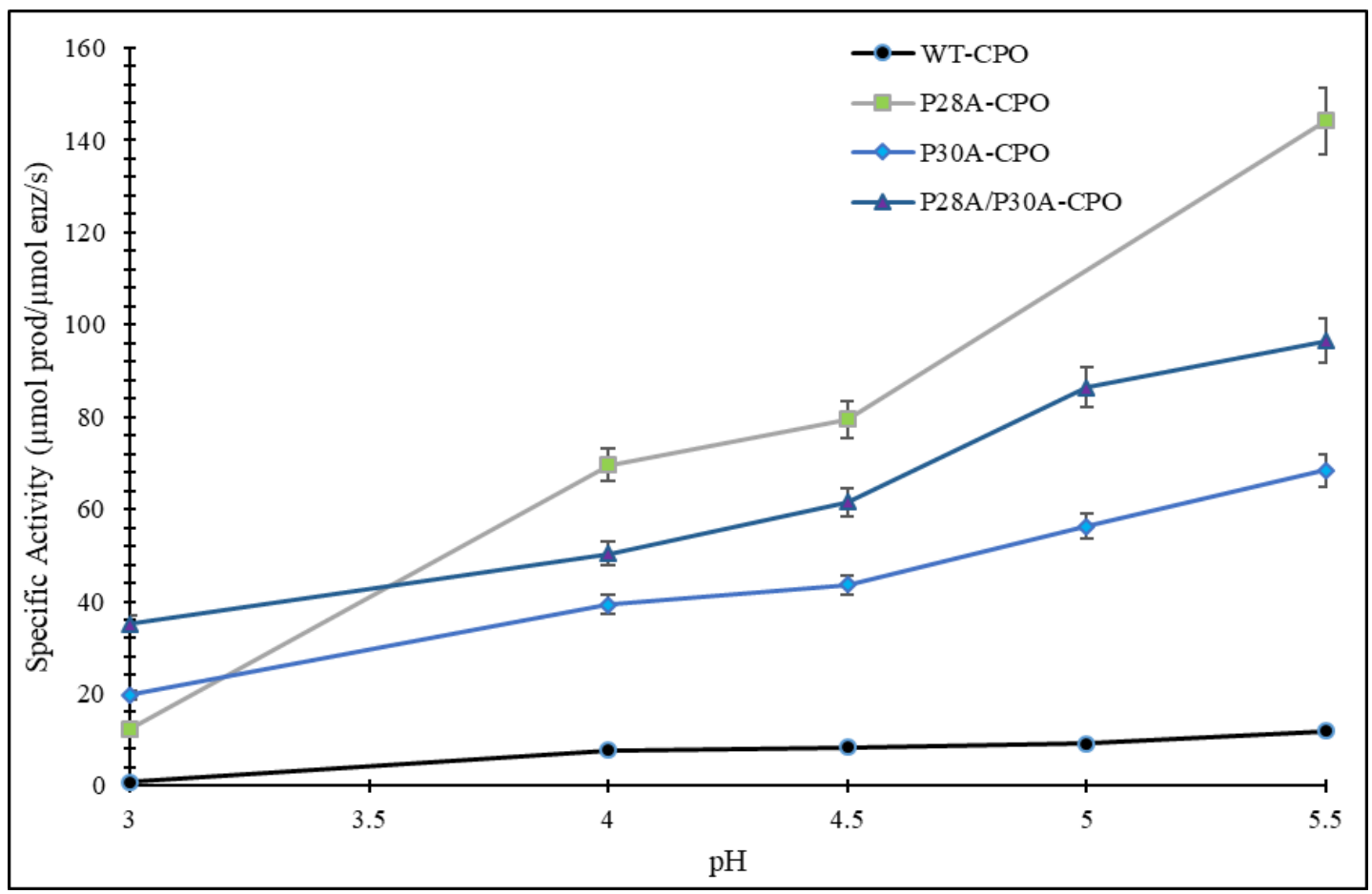

Figure 3.7 Specific activity of WT-, P28A-, P30A- and P28A/P30A-CPO for Epoxidation assay at pH 3.00-5.50. (Some error bars are not visible on the graph) 


\subsubsection{Dismutation of Hydrogen Peroxide}

Hydrogen peroxide is a common oxidant utilized by peroxidase to carried out oxidative reactions. However, excessive amount of this oxidant can lead to deleterious effects. A type of enzyme that remove excess oxidant is known as catalase. CPO's dismutation pathway limits the amount of hydrogen peroxide for the formation of Compound I. To test the catalatic activity of WT-, P28A-, P30A- and P28A/P30A-CPO, the rate of dismutation of hydrogen peroxide was measured by monitoring the change in the absorbance at $240 \mathrm{~nm}$ as observed in Figure 3.8. This study shown that WT-CPO's catalatic activity remains active throughout $\mathrm{pH} 2.00-6.00$. However, when $\mathrm{pH}$ falls below 2.00 , no activity was observed. As for P28A-CPO, no significant catalatic activity was observed within the tested $\mathrm{pH}$ range. Similar result was also observed for $\mathrm{P} 28 \mathrm{~A} / \mathrm{P} 30 \mathrm{~A}-\mathrm{CPO}$ where the activity is negatable as shown in Figure 3.9.

However, P30A-CPO retained significant catalatic activity only at $\mathrm{pH} 5.00$ and 6.00 . The results suggested that Pro28 has a significant contribution to the dismutation activity of $\mathrm{CPO}$ as mutation on this residue hindered the dismutation pathway as illustrated by P28A- and P28A/P30A-CPO. Pro30 play a subtler role relative to Pro28 in modulating the catalatic activity of $\mathrm{CPO}$, as the mutation only reduced the dismutation activity. The mutation(s) on Pro28 and Pro30 might influenced the helix-dipole moment resulting in the

decrease of the reduction potential of the mutants' Compound I intermediate thus prohibiting the oxidation of the second hydrogen peroxide. 


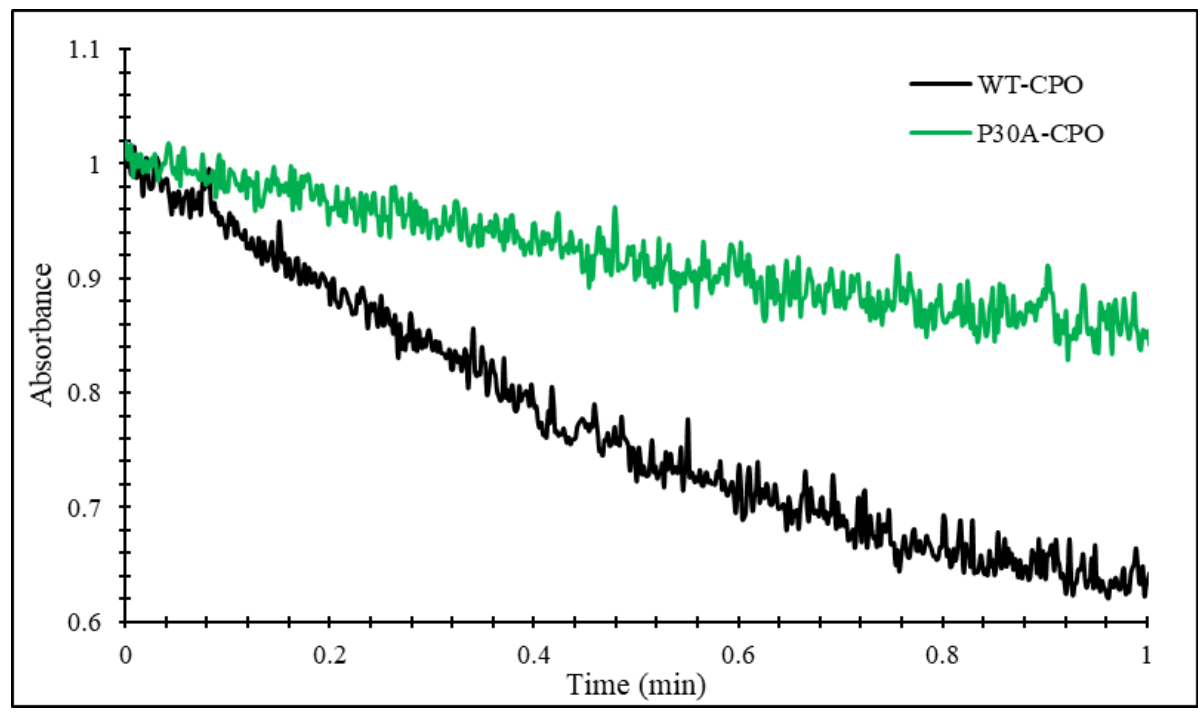

Figure 3.8 Dismutation of hydrogen peroxide catalyzed by WT- and P30A-CPO monitor at $240 \mathrm{~nm}$.

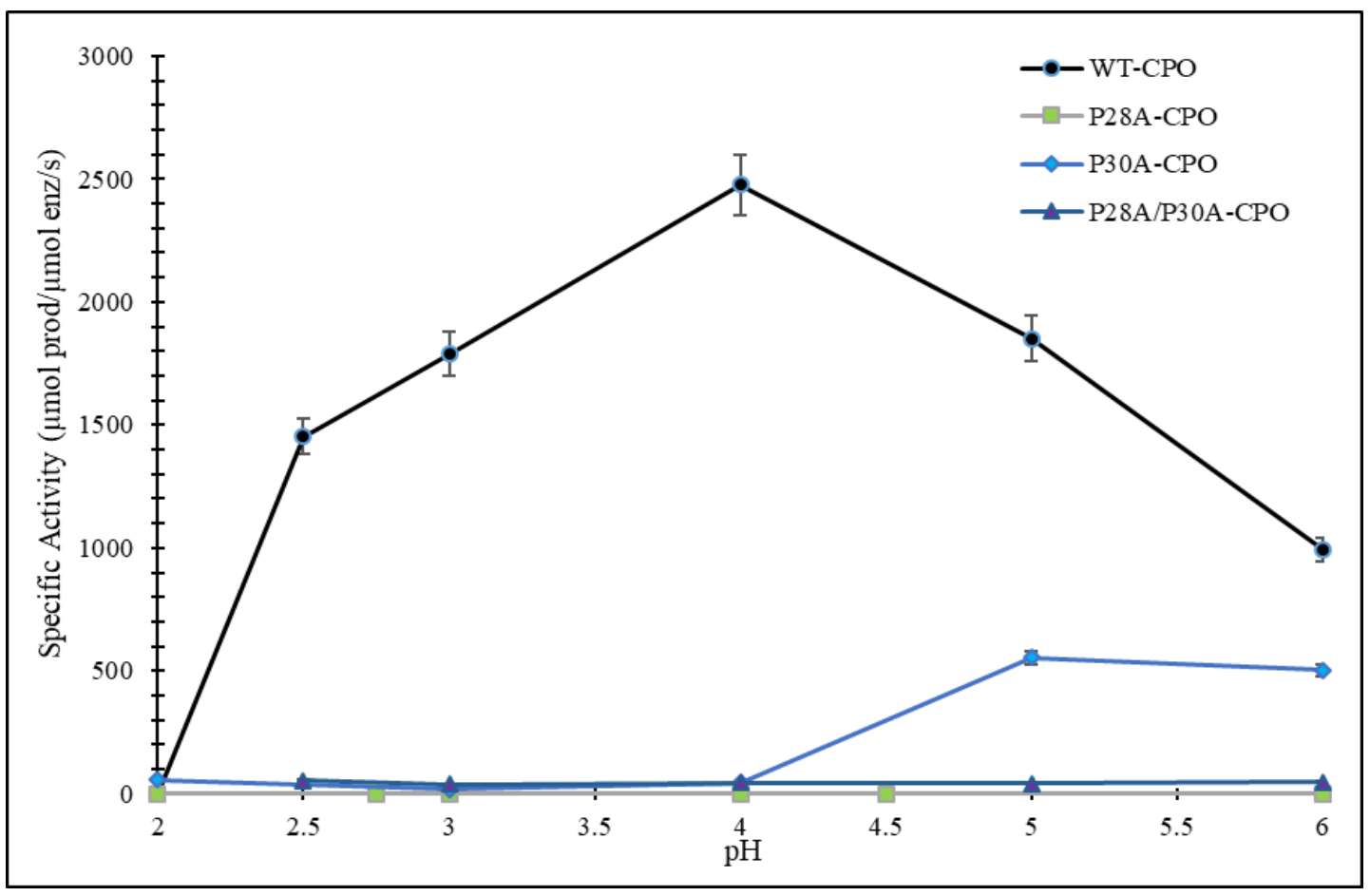

Figure 3.9 Specific activity of WT-, P28A-, P30A- and P28A/P30A-CPO for Epoxidation assay at pH 2.00-5.50. (Some error bars are not visible on the graph) 


\subsubsection{Halogenation of Monochlorodimedone}

MCD assay has been used to estimate the amount of CPO during the expression of this enzyme. In this assay, monochlorodimedone is chlorinated into dichlorodimedone which is measured through the decrease of absorbance at $278 \mathrm{~nm}$ as observed in Figure 3.10. WTCPO's chlorinating activity is most active at $\mathrm{pH} 2.75$ and the rate of the reaction decreased dramatically as $\mathrm{pH}$ rise. As for the mutants, no sign of chlorinating activity was observed (Figure 3.11). In this type of reaction, the redox potential of the halides need to be considered as whether the catalytic reaction can proceed. Considering the standards redox potential of the half reactions for hydrogen peroxide and the halides, as observed in Table 3.2. The oxidative capability of hydrogen peroxide is sufficient in oxidizing halides besides fluoride as no halogenase or haloperoxidase was known to catalyze fluorination reaction [72]. This suggested that CPO-I should contain a positive redox potential that is greater than that of hydrogen peroxide as CPO contains catalatic activity but lower than that of oxidizing fluoride. To investigate whether the redox potential of the CPO mutants was decreased and rendered the abolishment of the halogenating activity, the activity was further investigated with substituting the chloride ions in the MCD assay with bromide ions. 


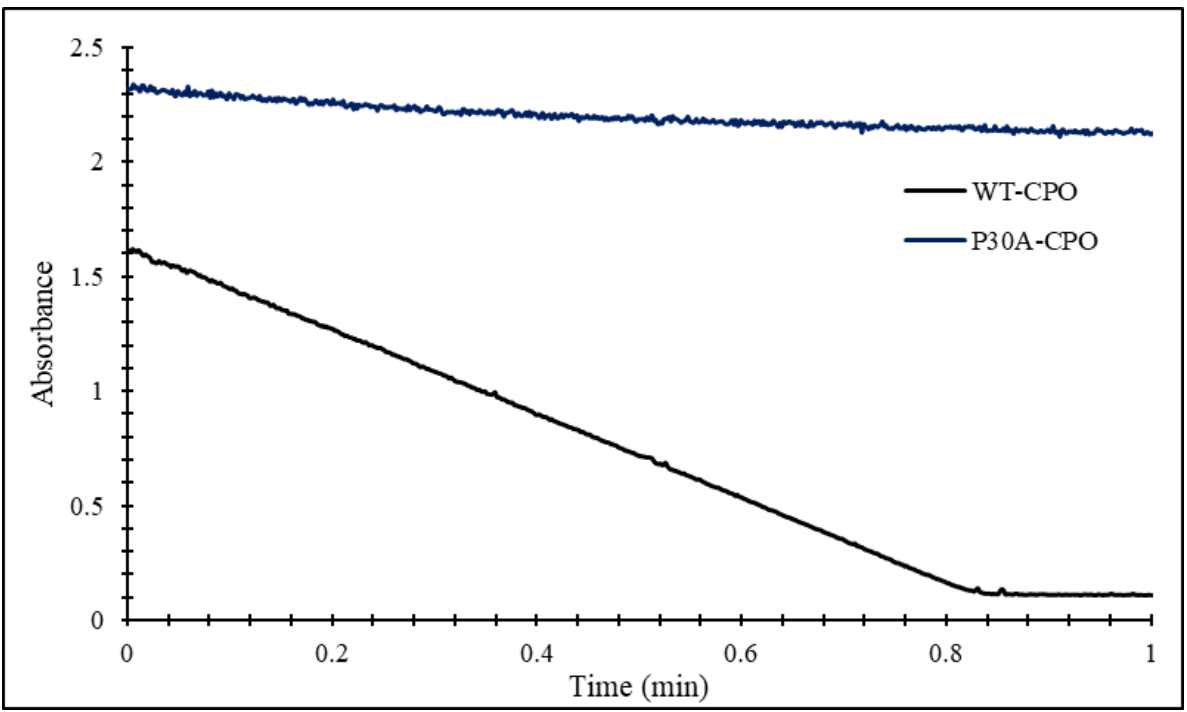

Figure 3.10 Chlorination of MCD catalyzed by WT- and P30A-CPO monitor at 278 nm.

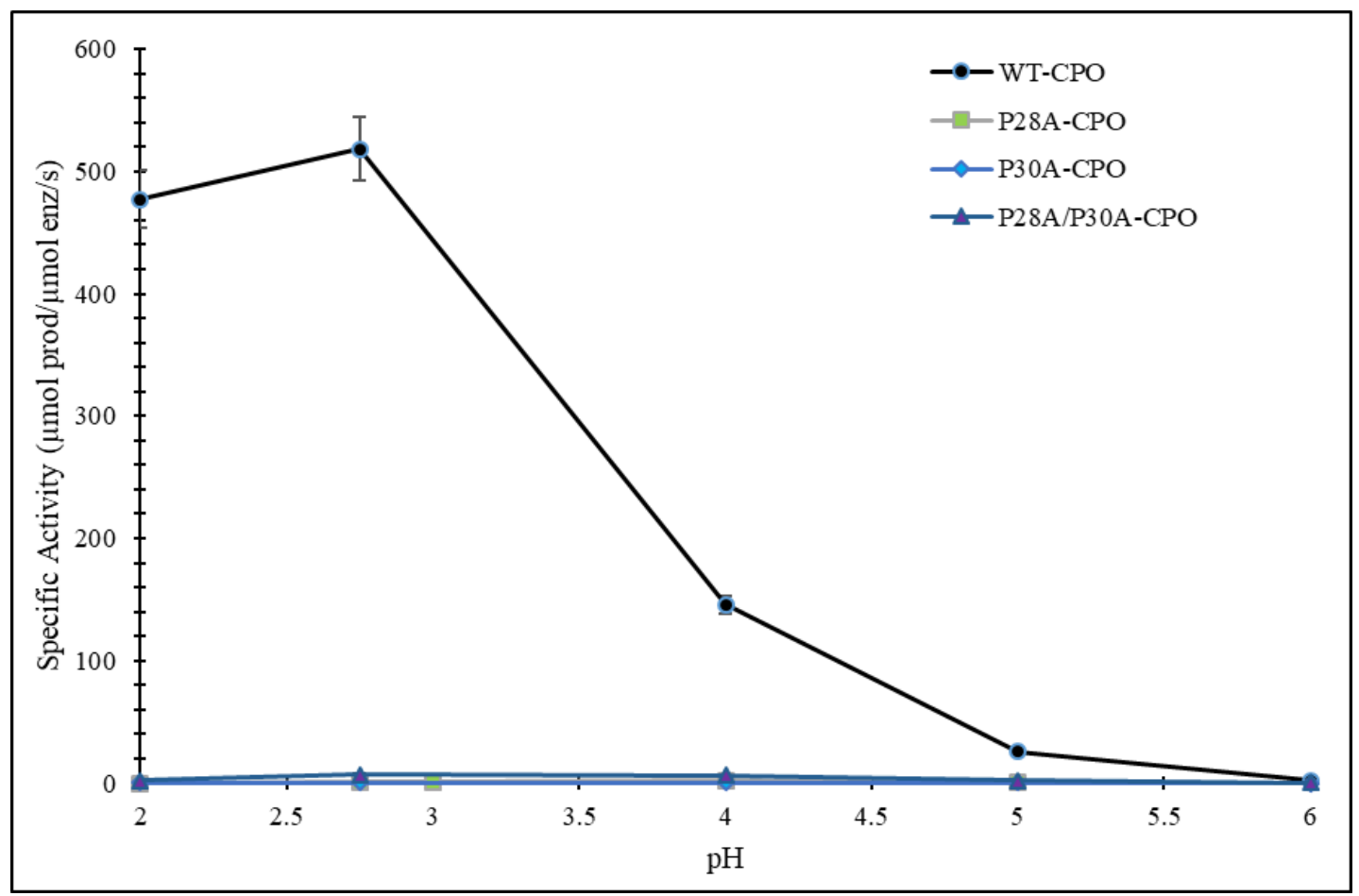

Figure 3.11 Specific activity of WT-, P28A-, P30A- and P28A/P30A-CPO for chlorination of MCD assay at pH 2.00-6.00. (Some error bars are not visible on the graph) 
Table 3.2 Half-reactions with redox potential of hydrogen peroxide and halide ions.

\begin{tabular}{|c|c|}
\hline Half-Reaction & $\mathrm{E}^{0}$, Volts \\
\hline $\mathrm{H}_{2} \mathrm{O}_{2}+2 \mathrm{H}^{+}+2 \mathrm{e}^{-} \rightarrow 2 \mathrm{H}_{2} \mathrm{O}$ & +1.77 \\
\hline $2 \mathrm{~F}^{-} \rightarrow \mathrm{F}_{2}+2 \mathrm{e}^{-}$ & -3.06 \\
\hline $2 \mathrm{Cl}^{-} \rightarrow \mathrm{Cl}_{2}+2 \mathrm{e}^{-}$ & -1.36 \\
\hline $2 \mathrm{Br}^{-} \rightarrow \mathrm{Br}_{2}+2 \mathrm{e}^{-}$ & -1.07 \\
\hline $2 \mathrm{I}^{-} \rightarrow \mathrm{I}_{2}+2 \mathrm{e}^{-}$ & -0.54 \\
\hline
\end{tabular}

As the reduction potential requires to oxidizes bromide ions is less than that of chloride ions, higher activity should be observed as seen in previous studies for WT-CPO [86]. Our result revealed the brominating activity of WT-CPO has about a five-fold increase relative to the chlorinating activity, which correlates with the previously reported data. However, the mutants still displayed no sign of halogenating activity after the bromide ions substitution (Figure 3.12). The result revealed that the CPO mutants lost all sign of halogenating activity. The plausible explanation might be that the redox potential has been reduced to a level where the oxidation of bromide ion is also prohibited. An alternate possibility for the demolish of the halogenation pathway for the CPO mutants might arise from the changes induced on the structure of the enzyme which resulted in the disruption of the halide-binding sites. However, the CD analysis showed no significant difference between WT-CPO and the CPO mutants and the mutation mainly focused on the proximal pocket where major changes in the halide-binding site seem to be less probable. 


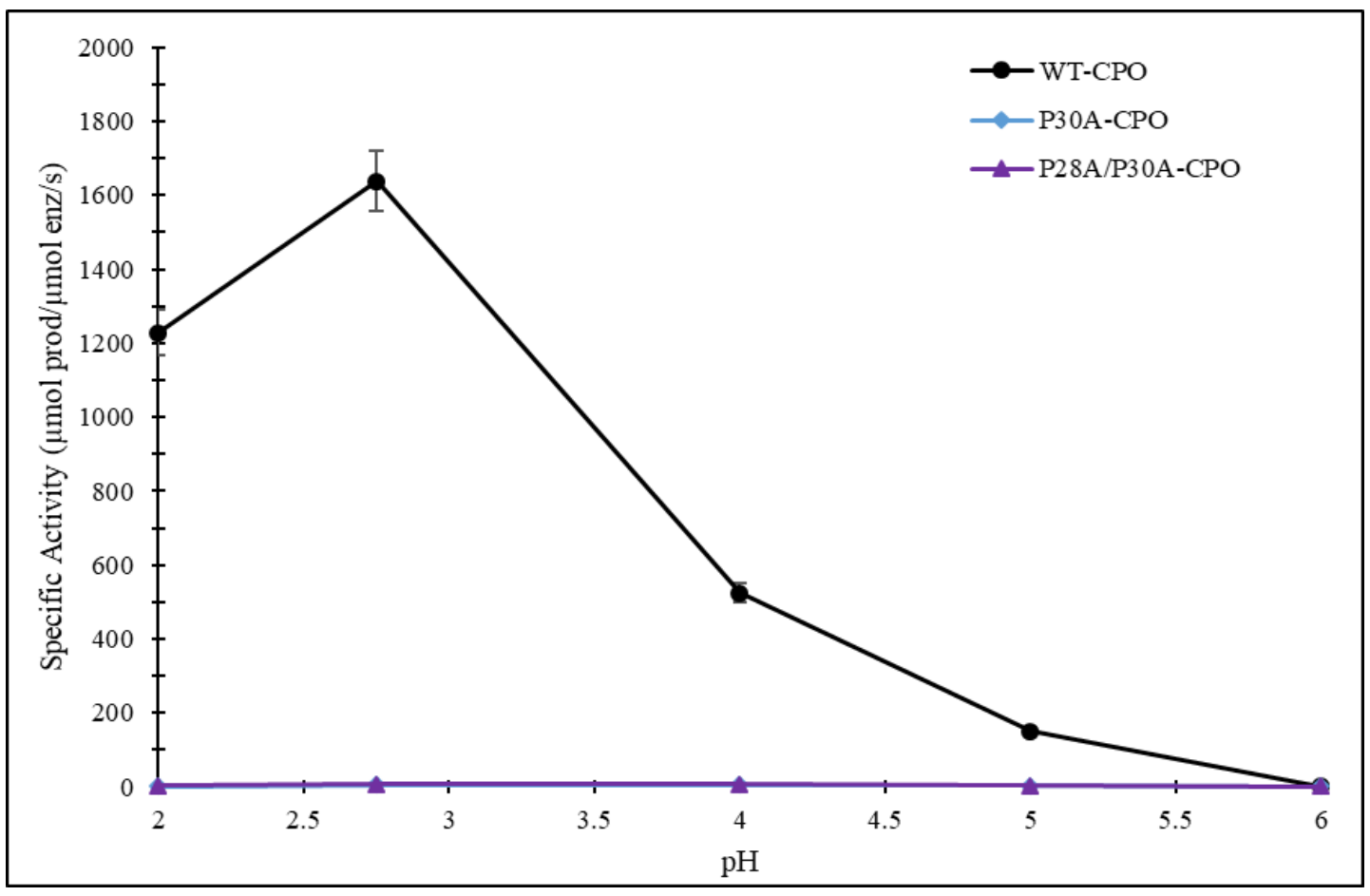

Figure 3.12 Specific activity of WT-, P30A- and P28A/P30A-CPO for bromination of MCD assay at pH 2.00-6.00. (Some error bars are not visible on the graph)

\subsubsection{Relative Activities}

P28A- and P28A/P30A-CPO contain no significant sign of chlorination and dismutation activities while retaining peroxidation and epoxidation activities. Similarly, P30A-CPO contains no sign of chlorination activity and enhanced peroxidation and epoxidation activities but significant dismutation activity at $\mathrm{pH} 5.00$ and 6.00 was observed. Therefore, specific $\mathrm{pH}$ (Table 3.3) for the four assays were chosen for comparison of the relative specific activities between WT-CPO and the CPO mutants. These $\mathrm{pH}$ values were selected because they are either commonly used in studies to their respective reactions or displayed significant difference between WT-CPO and the CPO mutants. 
Table 3.3 pH comparison for the Four Assays (ABTS, MCD, Epoxidation, and Dismutation).

\begin{tabular}{|l|l|}
\hline Assays & $\mathrm{pH}$ \\
\hline Epoxidation & 5.00 \\
\hline Dismutation & $5.00 \& 6.00$ \\
\hline MCD & 2.75 \\
\hline ABTS & $2.50 \& 4.00$ \\
\hline
\end{tabular}

Overall, my results suggested that the enhancement of the epoxidation pathway of the CPO mutants is at the expense of the dismutation activity as more of the reactive species, Compound I, and oxidizing agent is available for catalyzing the epoxidation reaction. The relative activity clearly demonstrated the competing relationship between the epoxidation and dismutation pathway as observed in Figure 3.13 and 3.14. The order of epoxidation activity from highest to lowest are P28A-, P28A/P30A-, P30A- and WT-CPO at pH 4.00 or higher. The epoxidation activity of P28A-, P30A- and P28A/P30-CPO were enhanced by 12-, 6-, and 9-fold relative to WT-CPO at pH 5.00, respectively.

At $\mathrm{pH} 5.00, \mathrm{P} 30 \mathrm{~A}-\mathrm{CPO}$ retained approximately $35 \%$ catalatic activity relative to WTCPO. Among the CPO mutants, P30A-CPO was the only mutant that retained partial catalatic activity, which explained why this CPO mutant displayed the least enhanced epoxidation activity. However, no significant catalatic activity was observed for the mutants at WT-CPO's optimal pH (4.00) (Figure 3.15). 


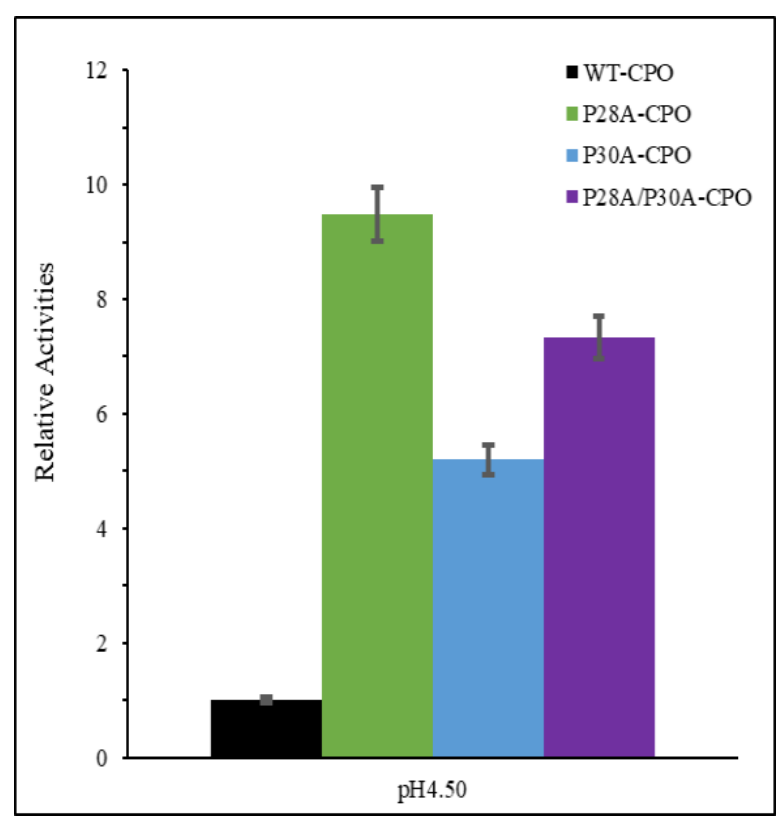

Figure 3.13 Relative activity of WT-, P28A-, P30A- and P28A/P30A-CPO for Epoxidation assay at $\mathbf{p H}=\mathbf{5 . 0 0}$.

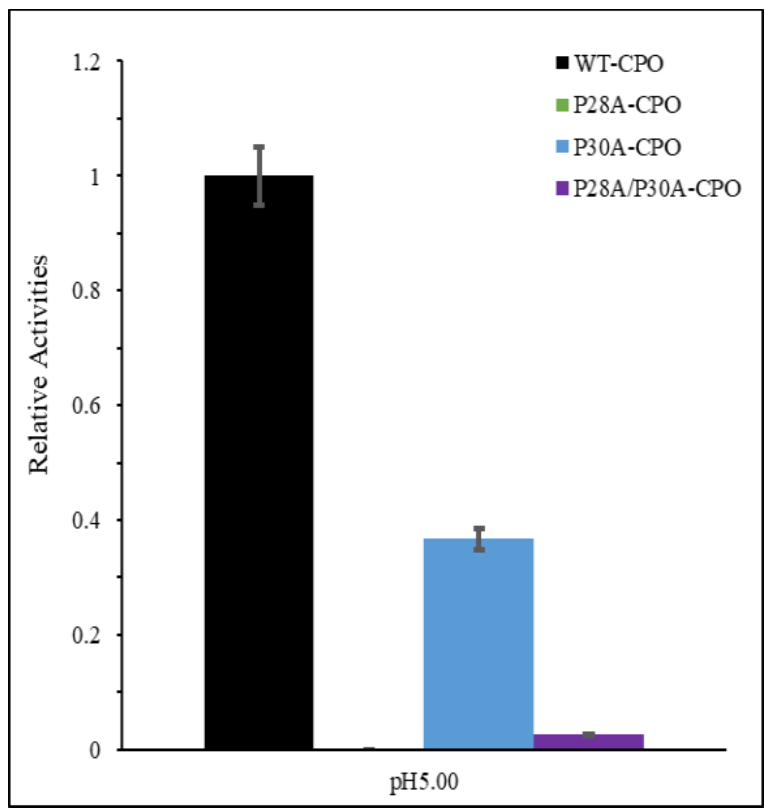

Figure 3.14 Relative activity of WT-, P28A-, P30A- and P28A/P30A-CPO for dismutation of hydrogen peroxide assay at $\mathbf{p H}=5.00$. 


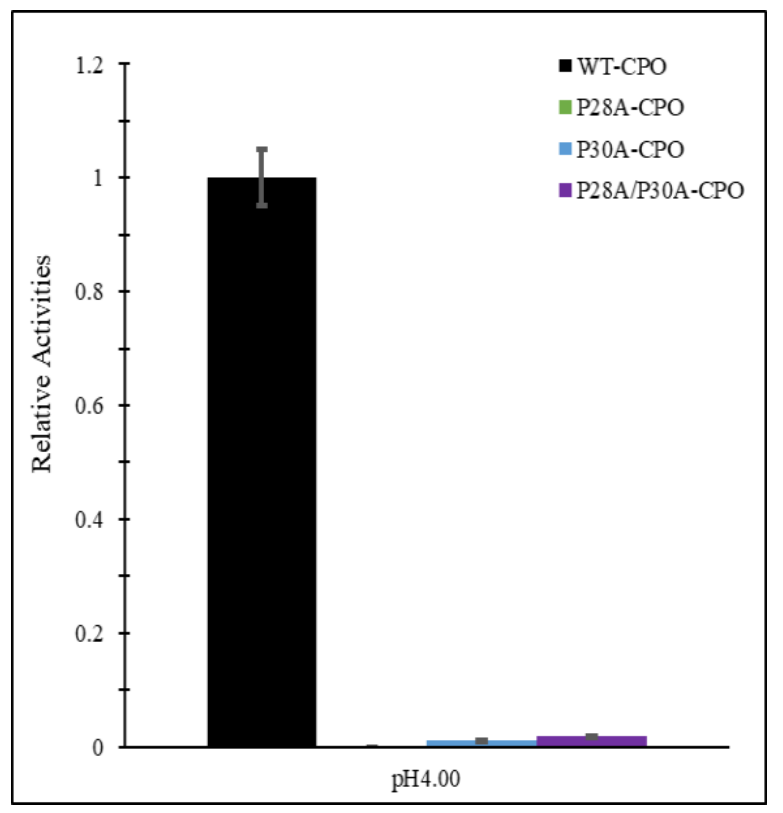

Figure 3.15 Relative activity of WT-, P28A-, P30A- and P28A/P30A-CPO for dismutation of hydrogen peroxide assay at $\mathrm{pH}=4.00$, optimal $\mathrm{pH}$ for WT-CPO.

As stated in Chapter I, the proposed mechanism of the dismutation pathway may involved the formation of the Compound I-peroxide species, which was crucial for the decomposition of hydrogen peroxide. The mutation(s) may hinder the formation of the Compound I-peroxide species thus result in the loss or reduction of the catalatic activity. The results for P28A-, P30A-, and P28A/P30A-CPO suggested that mutations within the Pro-Cys-Pro tripeptide might lead to the decrease in the redox potential of the heme center while increasing the basicity of the distal ligand. The possible decrease in the redox potential could explain the mutants' disability in oxidizing hydrogen peroxide.

At $\mathrm{pH} 2.75$, the optimum $\mathrm{pH}$ for $\mathrm{CPO}$-catalyzed chlorination reactions, no significant sign of activities for chlorination and bromination were observed for the CPO mutants as observed in Figure 3.16. The possible decrease in the redox potential of the CPO mutants might be the plausible explanation on the abolishment of chlorinating activity, as Compound I is inefficient in oxidizing chloride ions. MCD assay in the presence of 
bromide ion revealed that there was no significant difference between chlorination and bromination reaction (Figure 3.17). The approximate $0.3 \%$ relative chlorination and bromination activities observed for the mutants were from the direct oxidation of MCD [36].

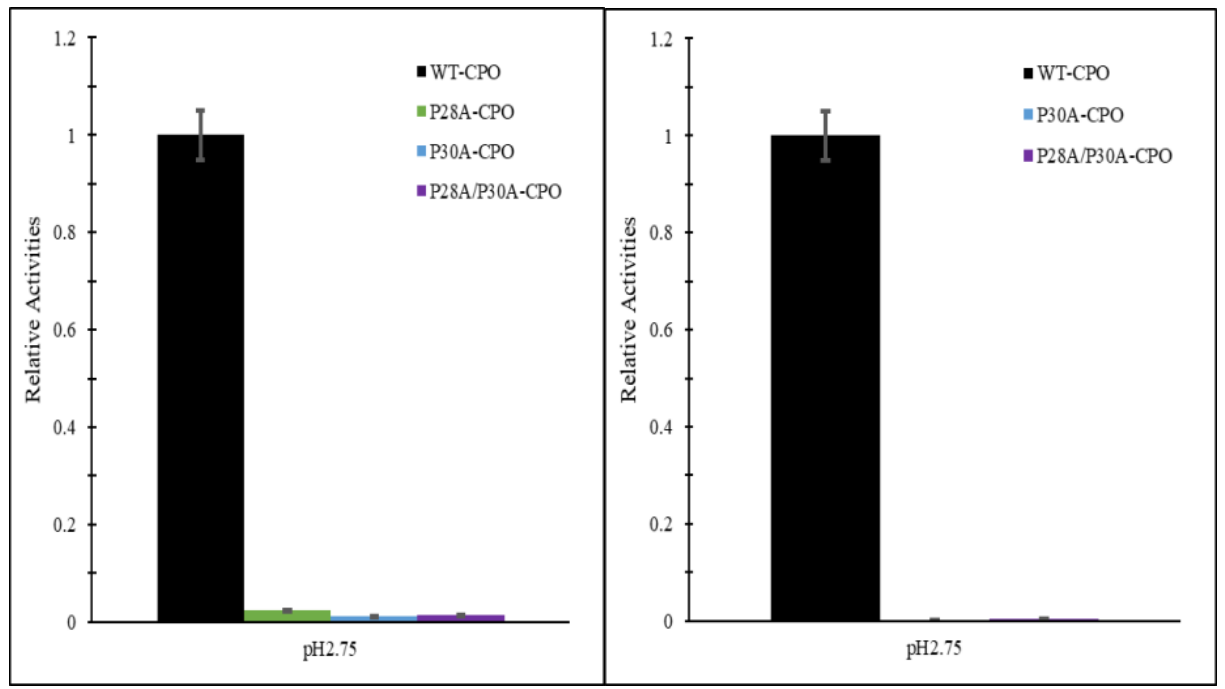

Figure 3.16 Relative activity of WT-, P28A-, P30A- and P28A/P30A-CPO for chlorination, Left, and bromination, Right, of MCD assay at pH=2.75.

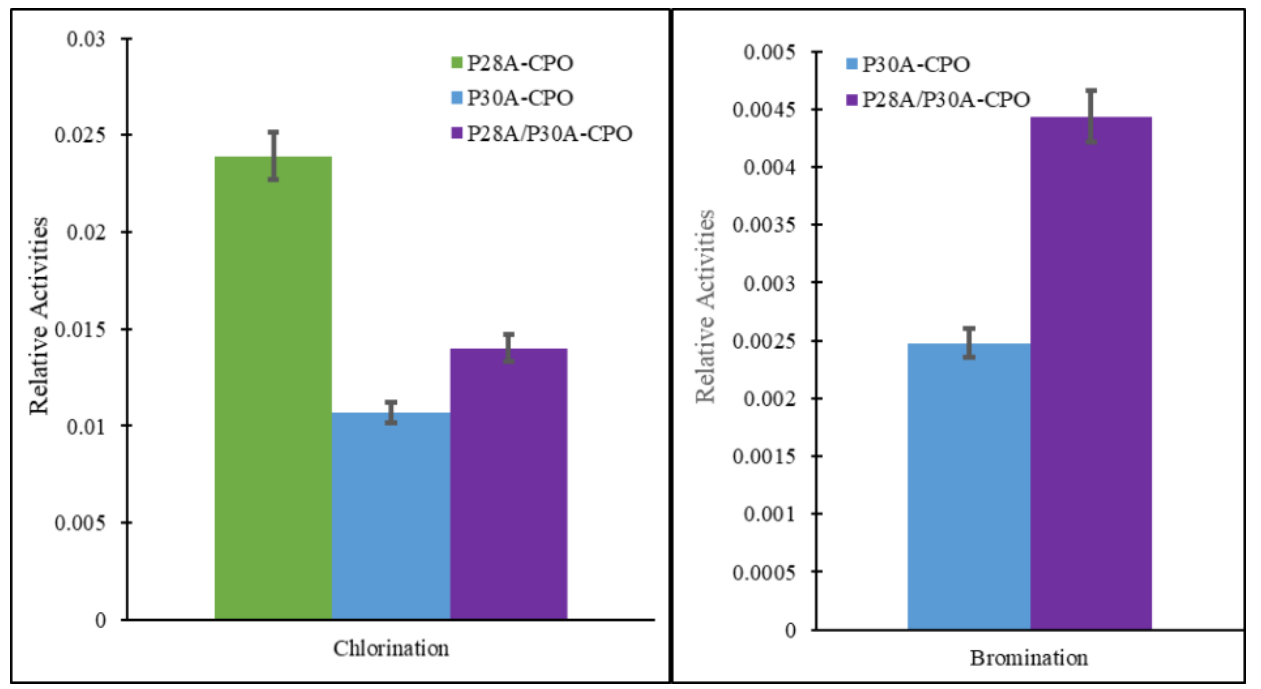

Figure 3.17 Relative activities of P28A-, P30A- and P28A/P30A-CPO for chlorination, Left, and bromination, Right, of MCD assay at $\mathrm{pH}=2.75$.

Both, dismutation and halogenation, pathways involves two-electrons oxidative reactions and the possible decrease in the redox potential of the CPO mutants hindered 
these two pathways. As the redox potential decreased, the $\mathrm{pK}$ a of the distal ligand increased favoring the protonation of Compound II. In the catalytic cycle of CPO, the pathway that involves Compound II is the peroxidation pathway, a two one-electron oxidation reaction. As shown in Figure 3.18 and 3.19, WT-, P28A-, P30A- and P28A/P30A-CPO displayed relativity different degree of peroxidase's activity.

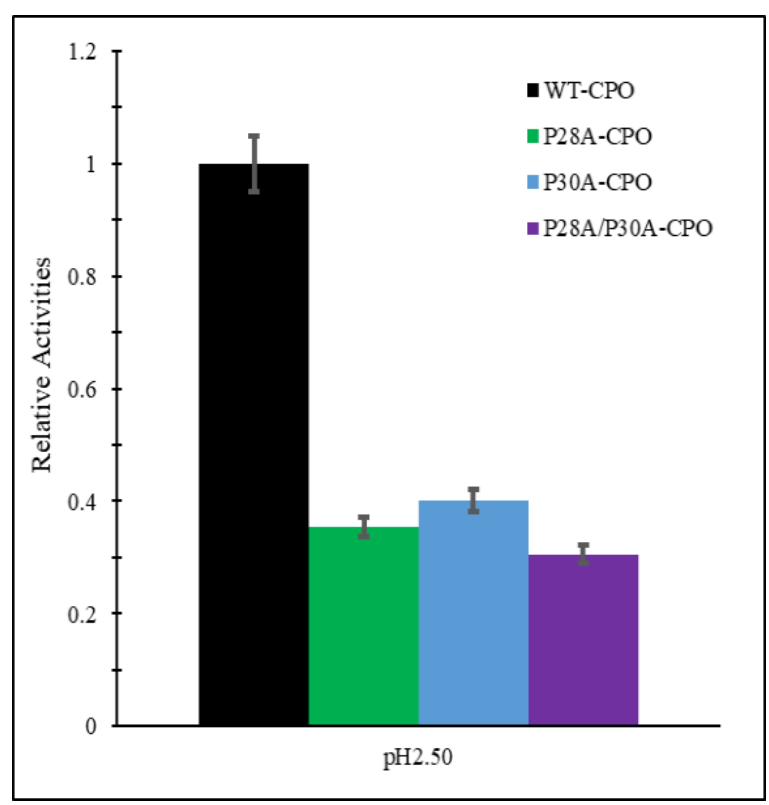

Figure 3.18 Relative activity of WT-, P28A-, P30A- and P28A/P30A-CPO for peroxidation of ABTS assay at $\mathrm{pH}=2.50$, optimal $\mathrm{pH}$ for WT-CPO.

At $\mathrm{pH} 2.50$, WT-CPO contains the highest peroxidation activity while the CPO mutants retained only about $30-40 \%$ of relative activity. However, the activity of the CPO mutants is significantly enhanced at $\mathrm{pH} 4.00$ or higher. For example, at $\mathrm{pH} 4.00$, the activities of P28A-, P30A- and P28A/P30A-CPO were 4-, 13- and 9-fold relative to WT-CPO, respectively. These enhancements might possibly be the result of the lack of catalatic activity as well as the increase in the basicity of the distal ligand. Our results suggested the $\mathrm{pK}_{\mathrm{a}}$ of Compound II for WT-CPO and the CPO mutants is different with the latter being 
higher. Therefore, at pH 4.00 or higher, Compound II of WT-CPO has a more difficult time forming the protonated species.

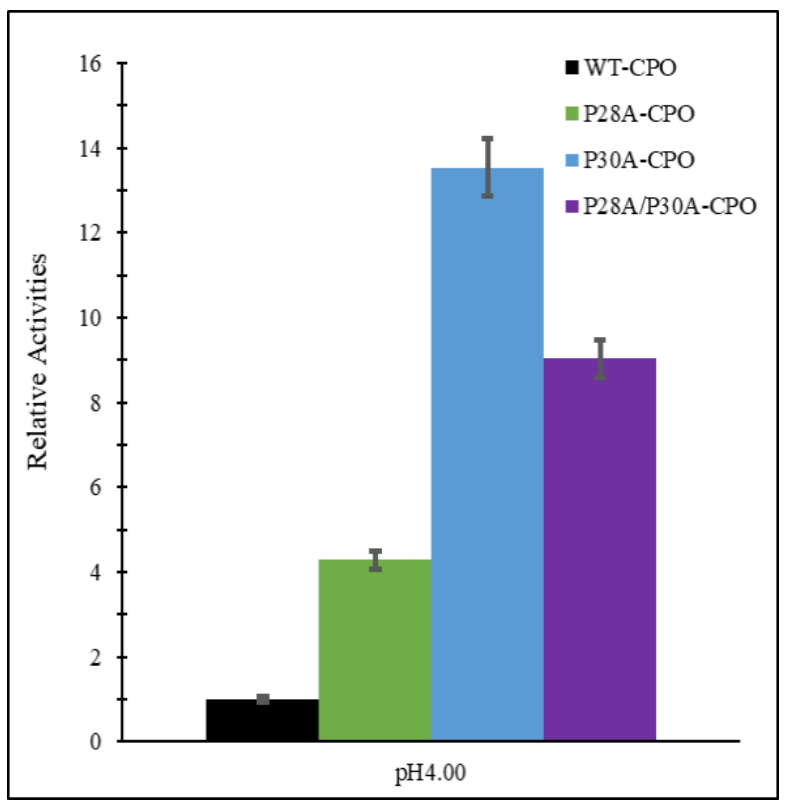

Figure 3.19 Relative activity of WT-, P28A-, P30A- and P28A/P30A-CPO for peroxidation of $\mathrm{ABTS}$ assay at $\mathrm{pH}=4.00$.

\subsection{Conclusion}

The proximal loop of CPO has been proposed to play an important in modulating the structural and biochemical properties of the enzyme. Within this loop, few distinctive features highlighted the significant of CPO. The proximal loop of CPO consists of hydrogen bonding networks that function in maintaining the structure of the helix as well as regulating the reactivity of the enzyme. The Pro28-Cys29-Pro30 tripeptide stretch containing the proximal ligand has been found to be conserved for heme-thiolate proteins such as UPOs. The importance of these proline residues was investigated in my research in which Pro28 and Pro30 in CPO were replaced with alanine to examine the effects on its biochemical properties. The results of my study revealed that these proline residues play an important role to regulating the catalytic properties of CPO. 
Our results indicated that mutation on Pro28 and/or Pro30 alter the optimal pH preference of CPO-catalyzed reactions. For example, the optimal $\mathrm{pH}$ for WT-CPO to catalyzes the peroxidation of ABTS is 2.50 but the optimal $\mathrm{pH}$ is shifted to 3.00 for P28A, P30A- and P28A/P30A-CPO. All three mutants possess no sign of chlorination activity. In term of hydrogen peroxide dismutation, P28A- and P28A/P30A-CPO contain no sign of catalatic activity while the activity of P30A-CPO was reduced. At the expense of the chlorination and dismutation activities, the epoxidation and peroxidation activities of the CPO mutants were enhanced. Especially, the epoxidation activity of the CPO mutants displayed with multi-fold enhancement at the tested $\mathrm{pH}$ range of 3.00-5.50 relative to WTCPO. Based on changes in the catalytic activities of CPO, Pro28 and Pro30 might play a role in regulating the redox potential of the heme center thus the mutation(s) resulted in the demolition of the dismutation and halogenation activities

Based on my results, the proline residues might regulate the reactivity of CPO through stabilizing the adjacent hydrogen bonding networks, amide-sulfur hydrogen bonding network and the proximal helix hydrogen bonding network. These hydrogen bonding networks have been proposed to regulates the "push" effect of the proximal ligand during the formation of Compound I as well as the redox potential of the catalytic heme center. The rigid nature of proline can stabilize these networks thus regulating activities such as chlorination and peroxide dismutation. If this is the case, then the individual hydrogen bonding network seems to contribute to a different extent to the catalytic properties of CPO. As the mutation on Pro28 abolished the catalatic activity while the P30A-CPO retained partial activity. Therefore, my research further our understanding on these conserved prolines that sandwiched the proximal cysteine as they are important in modulating the 
catalytic activity of $\mathrm{CPO}$, possibly through the stabilization of the adjacent hydrogen bonding networks. 


\section{CHAPTER IV.}

\section{MUTAGENESIS APPROACH ON THE SUBSTRATE-BINDING POCKET OF}

\section{CPO}

\subsection{Background}

On the surface of CPO, there are two channels where the substrate can diffuse into the active site. The bottom of the two channels form this substrate-binding pocket, which is located above the catalytic heme center [38]. The distal pocket of CPO resembles that of classical peroxidases as it is composed of polar residues. In heme peroxidases, the distal region contains a conserved His residue that act as an acid-base catalyst, which facilitates the cleavage of the peroxide bond during the formation of Compound I $[24,61,72,131$, 133]. However, the crystal structure of CPO revealed that the closest histidine, His 105, is still too far from the heme iron to catalyze the cleavage process. Instead, Glu183 is found to be the only polar/charged group within the distance to act as the acid-base catalyst [74]. Therefore, His105 is proposed to play a subtler role through the formation of a hydrogen bond with Glu183 and act as proton shuttle during the cleavage of the peroxide bond. This is also observed in classical peroxidases where instead of a histidine, an arginine residue nearby forms a hydrogen bond with His $[25,131]$.

In most heme peroxidases, substrate interactions occur through the heme edge while P450 enzymes contain a substrate-binding pocket that allow direct access to the ferryl-oxo center. CPO resembles to that of $\mathrm{P} 450$, as the substrate-binding pocket is located above the heme plane allowing the interactions between the substrate and the active site. The opening is guarded by the sidechains of three hydrophobic residues, Phe103, Phe186 and Val182 (Figure 4.1). These three residues have been proposed to interact with the substrate, 
orienting the substrate to a specific conformation, thus lead to the enantioselectivity of the reaction.

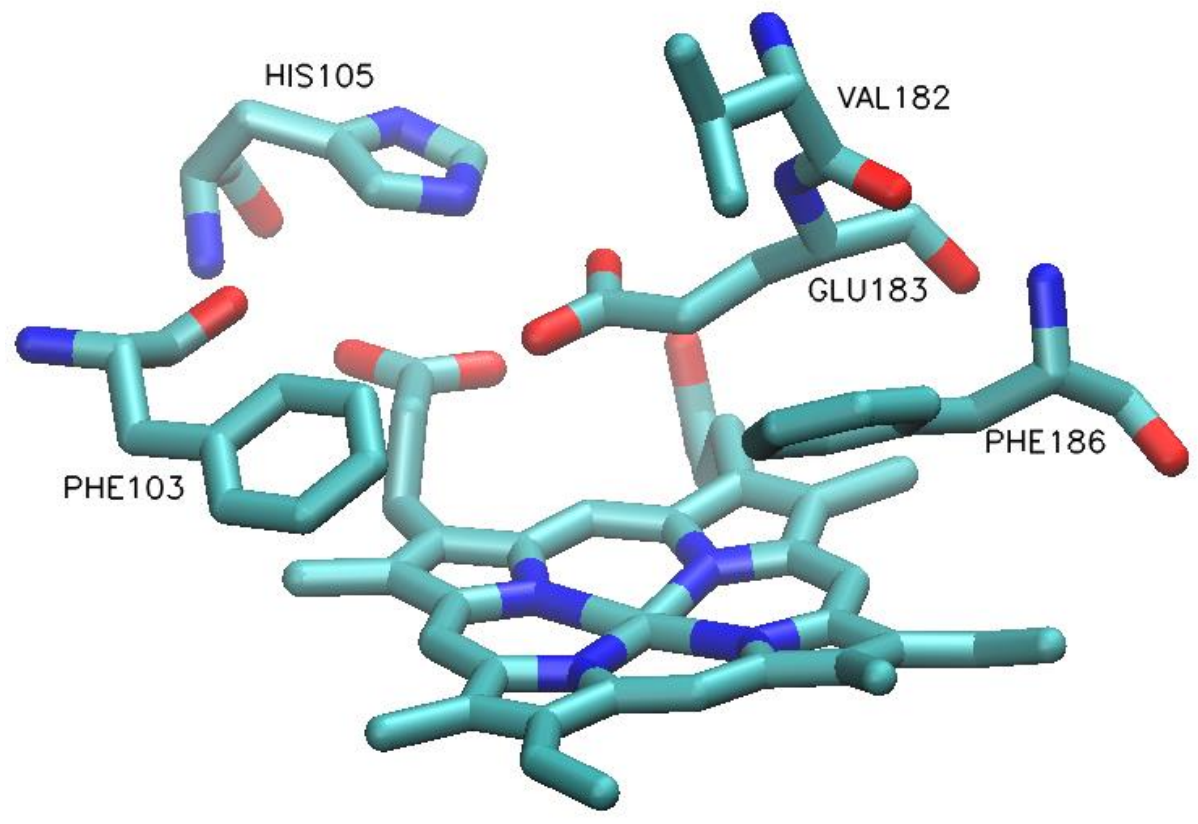

\section{Figure 4.1 Substrate-binding pocket on the distal region of CPO.}

Within our research lab, the contribution of Phe103 on the enantioselective reactions was previously examined. Through site-directed mutagenesis, Phe103 is replaced by an alanine residue increasing the space within the substrate-binding pocket of CPO thus allowing larger substrate to access the heme center. The result from our lab indicated that the distal pocket of CPO has a dramatic effect on the enzymatic activities as the mutant, F103A-CPO, displayed at least 4-fold enhancement of peroxygenase activity at the expense of the catalase activity. In term of enantioselectivity, F103A-CPO catalyzes epoxidation of long-side chain styrene derivatives with greater enantioselectivity relative to WT-CPO [124]. This mutant revealed the importance of Phe103 on the enantioselective reactions catalyzed by CPO. 
Phe103 is flexible in the substrate-binding pocket, as the binding of CPD to the active site of CPO resulted in the displacement of Phe103 by $0.5 \AA$ away from the active site to accommodate the substrate [70]. In addition, another phenylalanine, Phe186 has been proposed to play a similar role as Phe103; however, Phe186 is intact during the binding of CPD. To better understand the importance of Phe186 on CPO's catalysis, I investigated the role of Phe186 in the substrate-binding pocket through site-directed mutagenesis. In addition, a double mutant, F103A/F186A-CPO, was designed and characterized to further our understanding on the importance of these two residues on the effect on substrate selectivity and their enantioselectivity.

To investigate the substrate selectivity and their enantioselectivity, HPLC was employed for chiral separation of the extracted enantiomers. HPLC stand for high performance liquid chromatography and has been used for purifying chemicals and proteins [134]. Within liquid chromatography, there are two types of phases, stationary and mobile. The stationary phase is associated with the column of the HPLC and is selected based on the nature of the analyte. In this case, our study used the Whelk-O 1 column. Whelk-O 1 was selected because this column utilized a brush-type chiral stationary phase that function through a three-point interaction mechanism [135]. These interactions are $\pi$ $\pi$ stack formed with the dinitrophenyl group, hydrogen bonding interactions, and either $\mathrm{CH}-\pi$ interactions [136] or edge-to-face $\pi-\pi$ interactions [137]. In my study, hexane is used as mobile phase while the epoxide products of CPO-catalyzed epoxidation of styrene and its derivatives listed in Table 4.1 were extracted with isooctane. Therefore, the Whelk-O 1 column's tolerance against organic solvents as well as aqueous systems is crucial for my study. 
Table 4.1 Styrene and its derivatives and their corresponded epoxide products

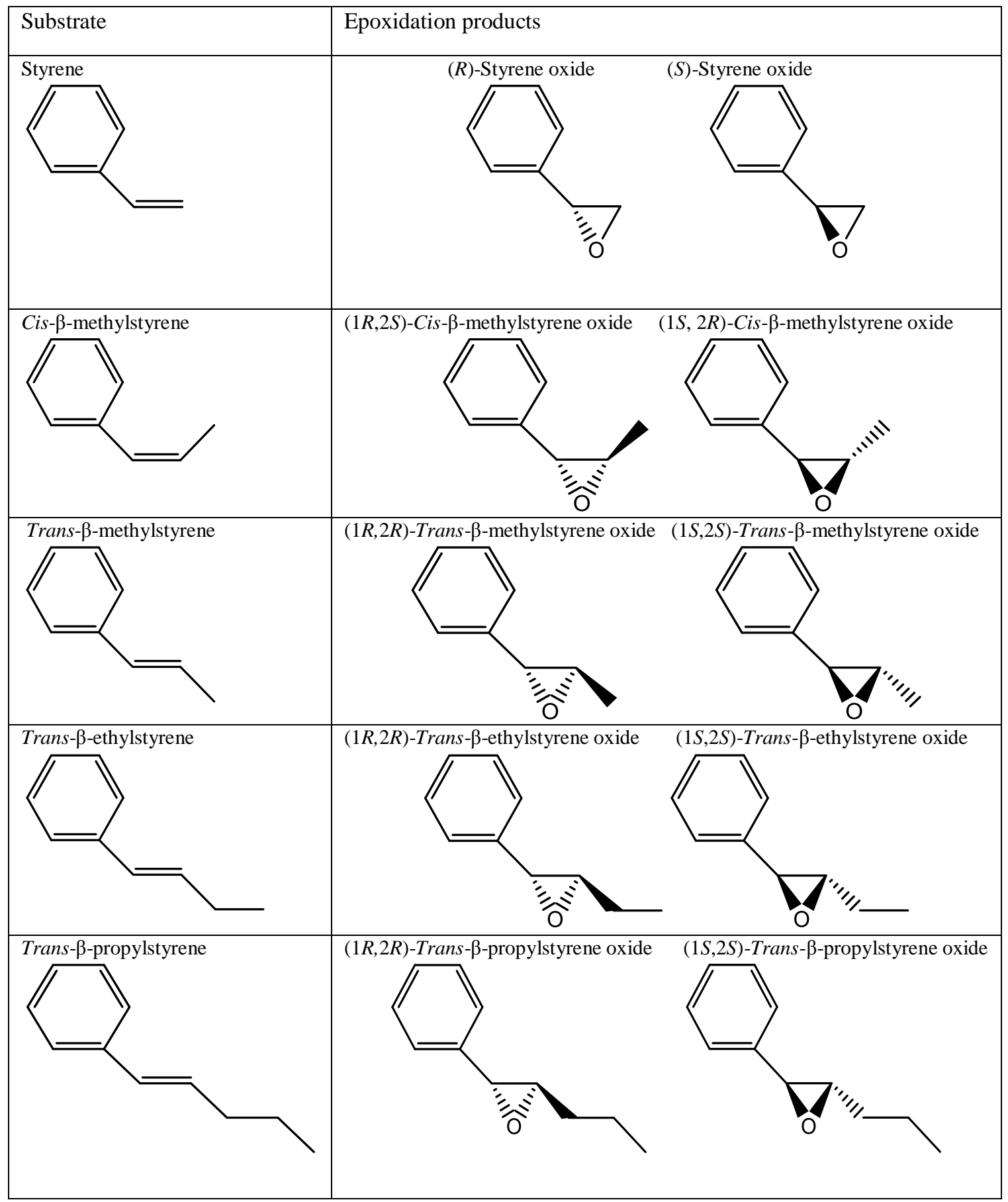




\subsubsection{Aim of this study}

In my study, Phe103 and Phe186 were mutated to an Ala residue, removing the phenyl sidechain, thus allowing bulkier substrate to bind to the pocket. Through site-directed mutagenesis, two CPO mutants, F186A- and F103A/F186A-CPO, were generated and characterized relative to WT-CPO and F103A-CPO. These mutants further our understanding on the importance of these two residues on the effect on the stereoselectivity of CPO-catalyzed epoxidation reactions as well as the selectivity of the substrates.

\subsection{Material and Methods}

\subsubsection{Construction of F186A- and F103A/F186A-CPO genes}

F186A- and F103A/F186A-CPO were constructed with procedure similar as those described in section 2.2.2. As for primers, they are listed in Table 4.2 for the two CPO mutants. DNA sequencing results of the two mutant plasmids were shown in Figure 4.2.

Table 4.2 CPO distal mutant's primers.

\begin{tabular}{|c|c|}
\hline Proximal Mutant CPO & Primers \\
\hline F186A-sense & 5'CGTCGAGTCTGGCGCCATCTTCGCCCTTG3' \\
\hline F186A-antisense & 5'GCGAAGATGGCGCCAGACTCGACGTTCTG3' \\
\hline F103A/F186A-sense & 5' CACGCTGCCGAGCACGACCACTC3' \\
\hline F103A/F186A-antisense & 5'TGCTCGGCAGCGTGGGGCTCG3' \\
\hline
\end{tabular}



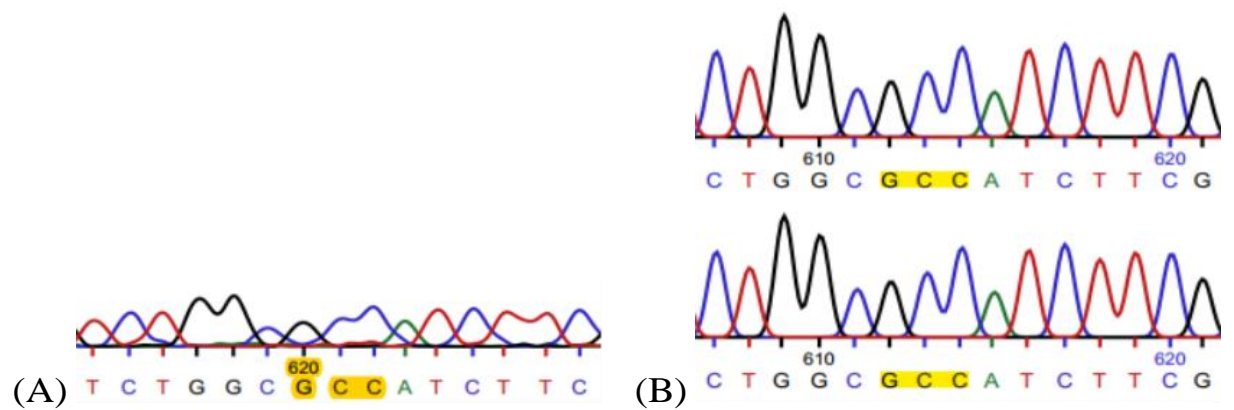

Figure 4.2 DNA Sequencing of (A) F186A- and (B) F103A/F186A-CPO

\subsubsection{Transformation, Expression and Purification of F103A- and F103A/F186A- CPO plasmid in Aspergillus niger}

The transformation process is similar as described in section 2.2.3 while the plasmids used were those with the distal region mutation. Successful transformants of F186A-CPO on the 1.2M Sorbitol plates are shown in Figure 4.3. As for the expression and purification process, the procedures were similar to that described in section 2.2.4.

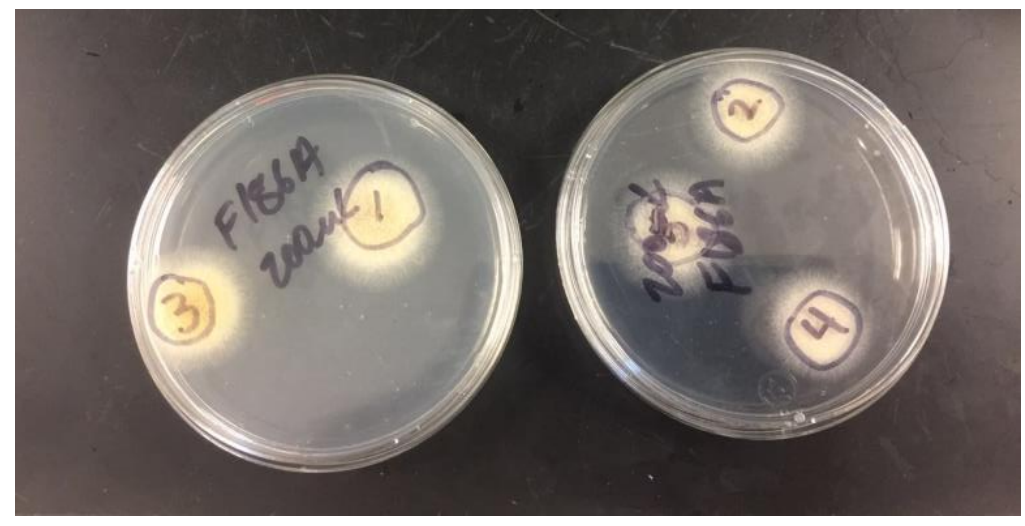

Figure 4.3 Successful transformants on 1.2 M Sorbitol acrylamide selective plate

\subsubsection{Spectroscopic Characterization}

The use UV-Vis spectrophotometry to characterize the mutant was similar to the procedure described in section 2.2.5. The ligand binding study for the distal region mutants were similar to the one described in section 2.2.6. In addition, $\mathrm{CD}$ spectrum was taken with similar procedure as section 2.2.7. 


\subsubsection{Enantioselective Study}

Styrene and its derivatives (cis- $\beta$-methylstyrene, trans- $\beta$-methylstyrene, trans- $\beta$ ethylstyrene, and trans- $\beta$-propylstyrene) were used as substrates to examine the enantioselectivity of WT-CPO and mutants catalyzed epoxidation reactions. A substrate mixture was prepared with $70 \mu \mathrm{L}$ of styrene or its derivatives and $100 \mu \mathrm{L}$ of tert-butyl hydroperoxide. The reaction was composed of $700 \mu \mathrm{L}$ of $100 \mathrm{mM}$ sodium citrate buffer, $\mathrm{pH} 5.50$, with about $2 \mathrm{mg}$ of $\mathrm{CPO}$ and was initiated by the addition of $25 \mu \mathrm{L}$ of the substrate mixture. After incubated at room temperature for 3 hours, the reaction was quenched by the addition of $50 \mu \mathrm{L}$ of $1 \mathrm{M} \mathrm{Na}_{2} \mathrm{~S}_{2} \mathrm{O}_{3}$ in a saturated solution of $\mathrm{NaHCO}_{3}$. The products were extracted with $300 \mu \mathrm{L}$ of isooctane and $20 \mathrm{uL}$ of the extracts were inject to a WhelkO1-SS column with $100 \%$ hexane as mobile phase. The flow rate was set at $1 \mathrm{~mL} / \mathrm{min}$ while monitoring the absorbance at $214-220 \mathrm{~nm}$.

\subsubsection{Data Analysis}

The HPLC results were analyzed with the following parameters:

$$
\text { Retention factor: } \mathrm{k}^{\prime}=\frac{\left(\mathrm{t}_{\mathrm{R}}-\mathrm{t}_{0}\right)}{\mathrm{t}_{0}}
$$

where $t_{R}$ is the retention time while $t_{0}$ is the void time;

$$
\text { Separation factor: } \alpha=\frac{k_{2^{\prime}}}{k_{1^{\prime}}}=\frac{t_{R 2}-t_{0}}{t_{R 1}-t_{0}}
$$

where $\mathrm{k}_{1}$ ' and $\mathrm{k}_{2}$ ' represented the retention factors of the two eluted enantiomers and $\mathrm{t}_{\mathrm{R} 1}$ and $t_{R 2}$ were the retention times for the two enantiomers;

$$
\text { Resolution factor: } \mathrm{R}_{\mathrm{S}}=\frac{2\left(\mathrm{t}_{\mathrm{R} 2}-\mathrm{t}_{\mathrm{R} 1}\right)}{\left(\mathrm{W}_{1}+\mathrm{W}_{2}\right)}
$$

where $\mathrm{W}_{1}$ and $\mathrm{W}_{2}$ represented the individual peak widths.

$$
e e=\% \text { (major isomer) }-\% \text { (minor isomer) }
$$




\subsection{Results}

\subsubsection{Circular Dichroism Spectroscopy of WT-, F186A-, and F103A/F186A-CPO}

The secondary structure composition of the CPO mutants was determined through circular dichroism spectroscopy. CD-spectra of WT-, F186A-, and F103A/F186A-CPO were shown in Figure 4.4. As observed in Table 4.3, the difference in the secondary structure of the proteins was not significant. The secondary structure of WT-CPO was comprised of approximately $34 \%$ and $22 \%$ of alpha-helices and beta-sheet, respectively. As for the CPO mutants, F186A- and F103A/F186A-CPO have a similar secondary composition of $32 \%$ alpha-helices and $21 \%$ beta-sheets. The difference between the WTCPO and the mutants was less than 2\%. Therefore, the mutation(s) of Phe103 and Phe186 to a smaller residue, Ala, does not significantly alter the secondary structure of the protein.

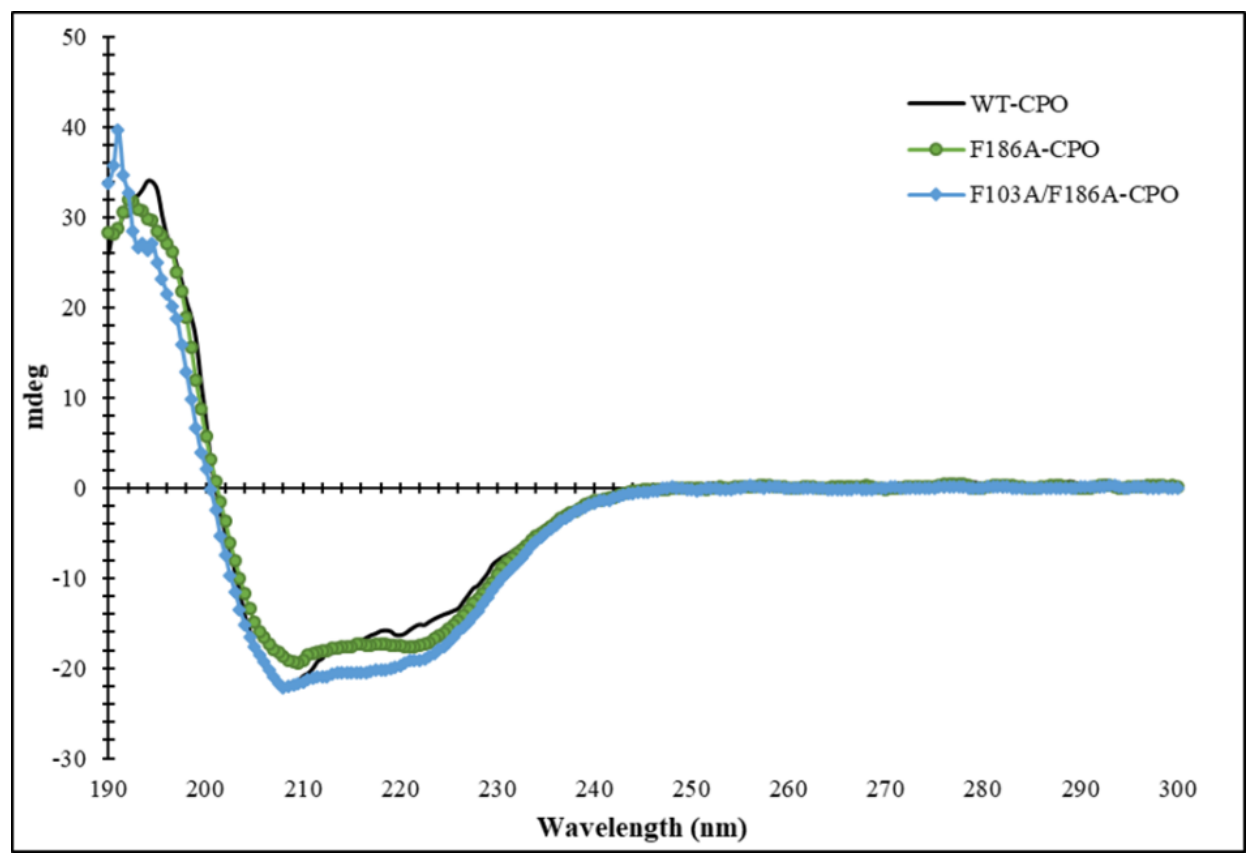

Figure 4.4 Secondary CD Spectra of $1.3 \mu \mathrm{M}$ WT-, F186A-, and F103A/F186A-CPO in $25 \mathrm{mM}$ phosphate buffer, pH 5.90. 
Table 4.3 Secondary Structure Composition of WT-, F186A-, and F103A/F186A-CPO in $25 \mathrm{mM}$ phosphate buffer $\mathrm{pH}=5.90$.

\begin{tabular}{|c|c|c|}
\hline Protein & $\alpha$-helix & $\beta$-sheet \\
\hline WT-CPO & $34 \%$ & $22 \%$ \\
\hline F186A-CPO & $32 \%$ & $21 \%$ \\
\hline F103A/F186A-CPO & $32 \%$ & $21 \%$ \\
\hline
\end{tabular}

\subsubsection{UV-Visible spectroscopic properties of WT-, F186A-, and F103A/F186A- CPO and their carbonmonoxy complex}

CD spectroscopy revealed no significant difference between WT-, F186A-, and F103A/F186A-CPO. However, the UV-Visible spectroscopic characterization showed otherwise. The UV-Visible spectrum of WT-CPO revealed that the enzyme exists as a penta-coordinated high-spin state as observed in Figure 4.5. This ferric enzyme was reduced to ferrous state through the addition of sodium dithionite where the Soret peak at $398 \mathrm{~nm}$ was shifted to $408 \mathrm{~nm}$ as an indication of the reduction. After reduction, carbon monoxide can bind to the ferrous WT-CPO producing the signature spectrum of hemethiolate proteins where the Soret peak absorbed at $445 \mathrm{~nm}$.

As observed in Figure 4.5 and 4.6, ferric F186A-CPO's Soret peak absorbed at $420 \mathrm{~nm}$ and this shift indicated the presence of a distal ligand as well as a hexa-coordinated lowspin species. Upon reduction, similar spectrum was observed for ferrous F186A-CPO as to ferrous WT-CPO. The mutation did not alter the identity of the proximal ligand as the CObound complex of F186A-CPO resemble that of WT-CPO-CO complex as well as other heme-thiolate proteins CO-bound complexes [27, 37, 138]. However, the resting state of the mutant resemble that of $\mathrm{P} 450$ as a low-spin species. As for F103A/F186A-CPO, similar spectra relative to F186A-CPO were observed for the carbon monoxide binding process as seen in Figure 4.7. Detail of the peaks for WT-, F186A-, and F103A/F186A-CPO as well 
as the ferrous and CO-bound forms were summarized in Table 4.4.

Table 4.4 UV-Vis spectral properties of ferric, ferrous and CO-bound WT-CPO and CPO mutants.

\begin{tabular}{|l|l|l|}
\hline Proteins & Soret band & $\beta$-band \\
\hline Ferric WT-CPO & $398 \mathrm{~nm}$ & $516 \mathrm{~nm}$ \\
\hline Ferrous WT-CPO & $408 \mathrm{~nm}$ & $546 \mathrm{~nm}$ \\
\hline Ferrous-CO complex WT-CPO & $445 \mathrm{~nm}$ & $550 \mathrm{~nm}$ \\
\hline Ferric F186A-CPO & $420 \mathrm{~nm}$ & $541 \mathrm{~nm}$ \\
\hline Ferrous F186A-CPO & $408 \mathrm{~nm}$ & $551 \mathrm{~nm}$ \\
\hline Ferrous-CO complex F186A-CPO & $445 \mathrm{~nm}$ & $549 \mathrm{~nm}$ \\
\hline Ferric F103A/F186A-CPO & $419 \mathrm{~nm}$ & $543 \mathrm{~nm}$ \\
\hline Ferrous F103A/F186A-CPO & $406 \mathrm{~nm}$ & $547 \mathrm{~nm}$ \\
\hline Ferrous-CO complex F103A/F186A-CPO & $444 \mathrm{~nm}$ & $549 \mathrm{~nm}$ \\
\hline
\end{tabular}

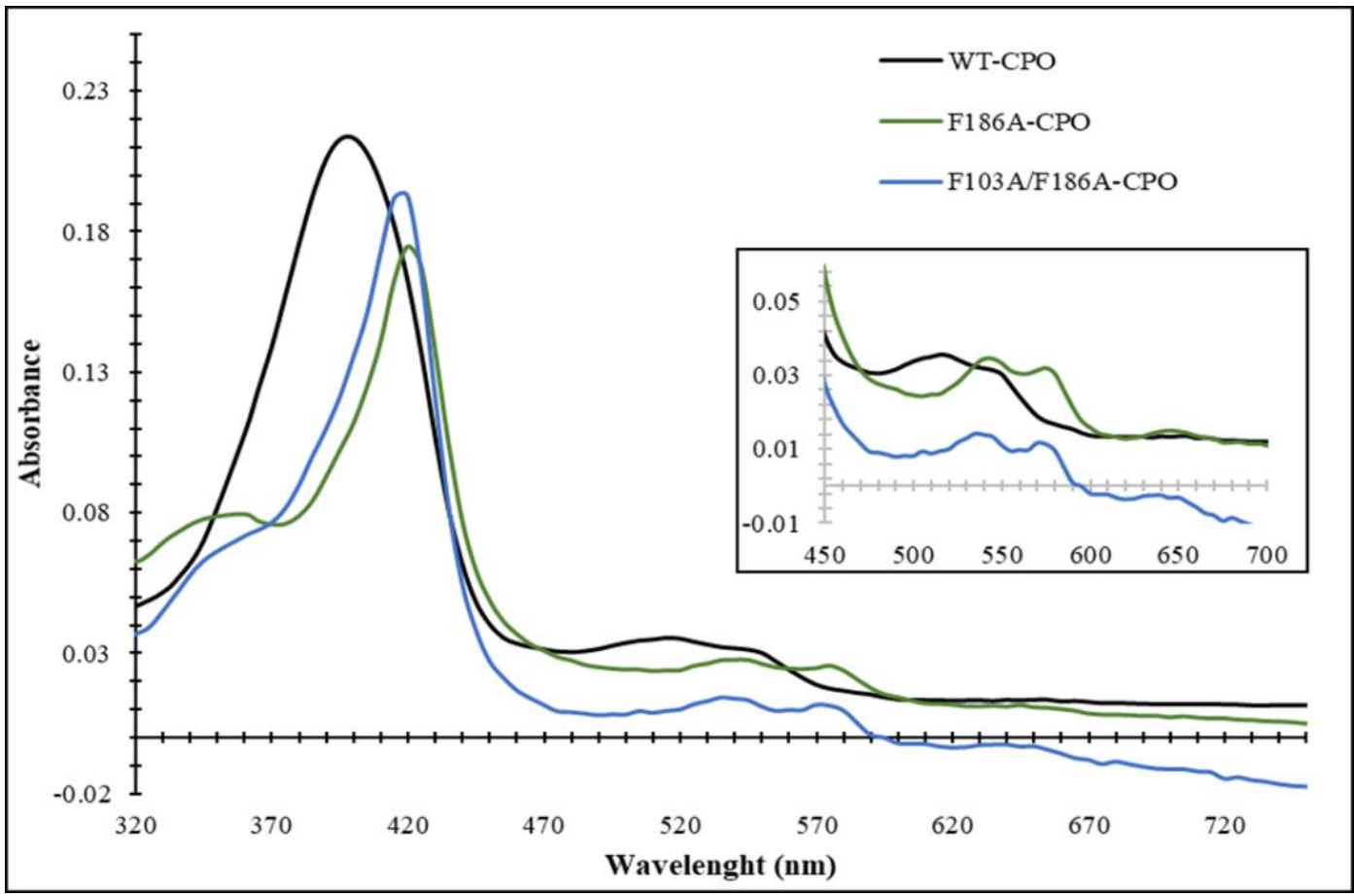

Figure 4.5 UV-Vis spectra of WT-, F186A-, and F103A/F186A-CPO in $25 \mathrm{mM}$ phosphate buffer, pH 5.90. 


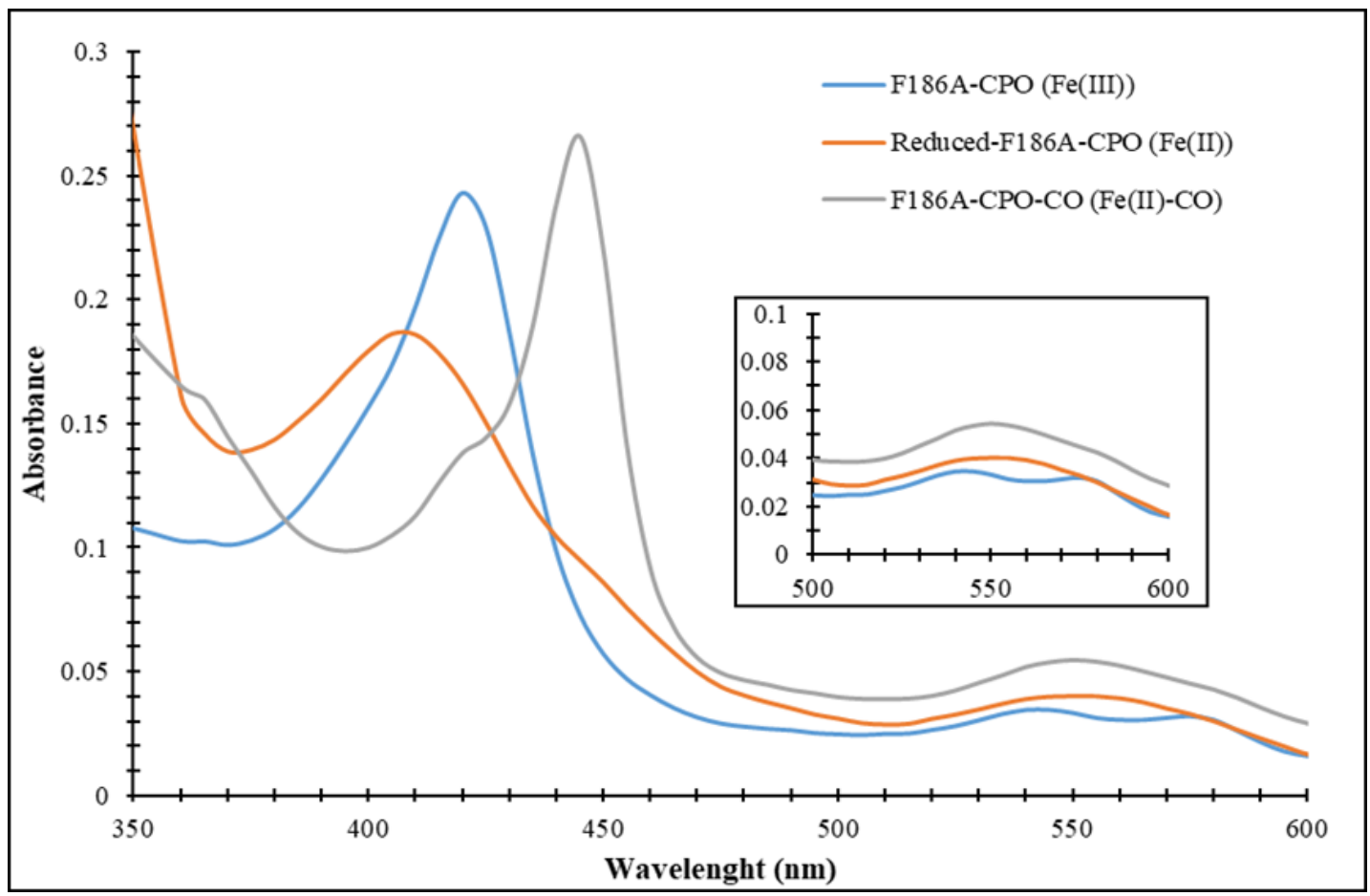

Figure 4.6 UV-Vis spectra of ferric, ferrous, and CO-bound F186A-CPO in $25 \mathrm{mM}$ phosphate buffer, pH 5.90.

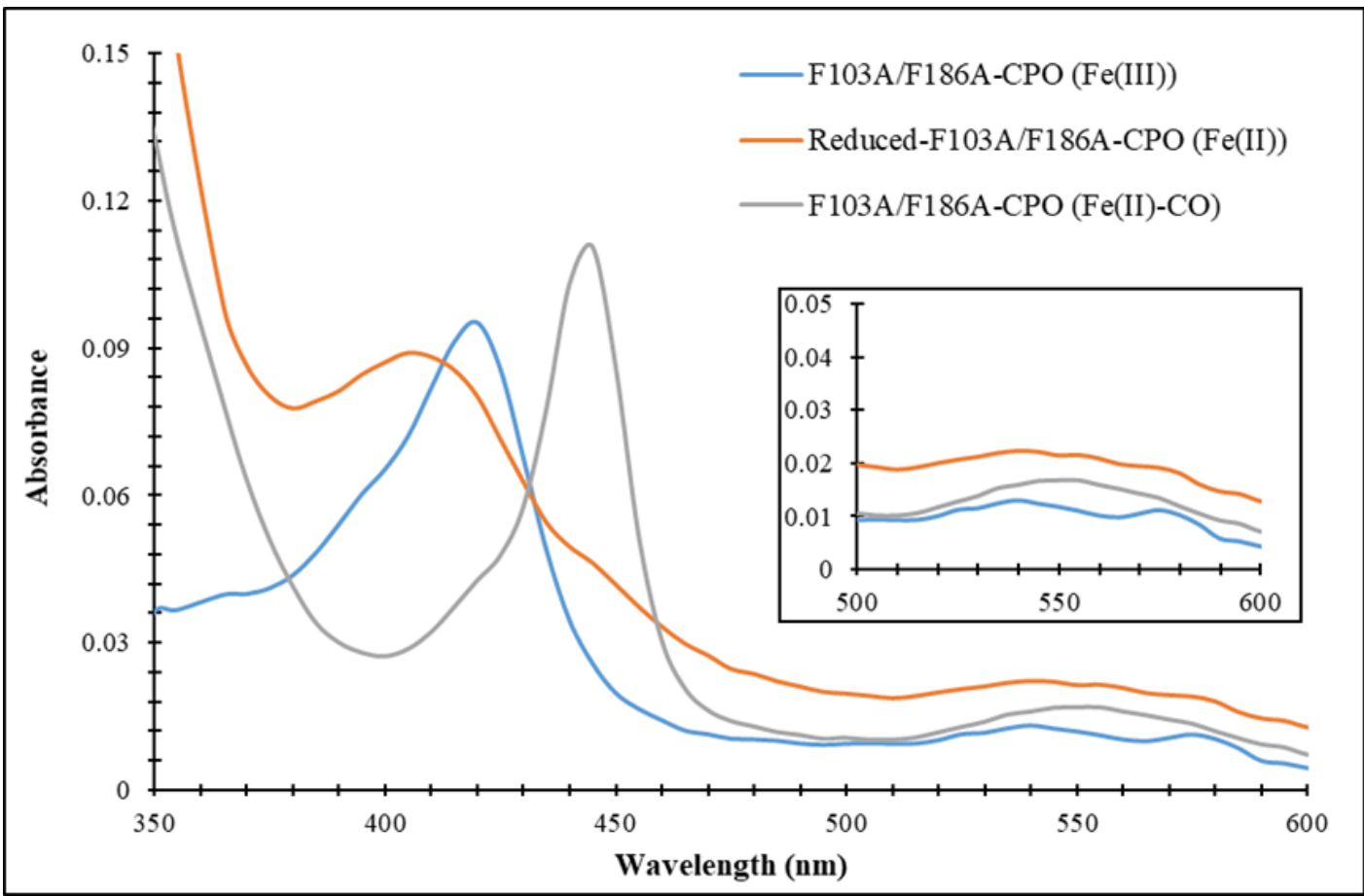

Figure 4.7 UV-Vis spectra of ferric, ferrous, and CO-bound F103A/F186A-CPO in 25 mM phosphate buffer, pH 5.90. 


\subsubsection{Cyanide Binding Study on WT-, F186A-, and F103A/F186A-CPO}

Cyanide bind to WT-CPO with high affinity, as stated in Chapter 2, the $\mathrm{K}_{\mathrm{d}}$ is 0.068 $\mathrm{mM}$. Titration of cyanide was performed on the $\mathrm{CPO}$ mutants and the $\mathrm{K}_{\mathrm{d}}$ was calculated. My result revealed F186A-CPO has a dissociation constant of $6.3 \mathrm{mM}$ for cyanide (Figure 4.8). The decrease in the binding affinity could be the result of a wider active site as well as the presence of a sixth ligand, which competes with the exogenous ligand. This explanation correlates with a previous study in our lab, that the CPO mutant, F103A-CPO, also had a higher $\mathrm{K}_{\mathrm{d}}$ of $4.5 \mathrm{mM}$ for cyanide [124]. Interestingly, the double, F103A/F186A, mutant displayed a $\mathrm{K}_{\mathrm{d}}$ of $3.6 \mathrm{mM}$ which is lesser than that of the two single mutation mutants. However, this $\mathrm{K}_{\mathrm{d}}$ value is still significantly greater than that of WT-CPO.

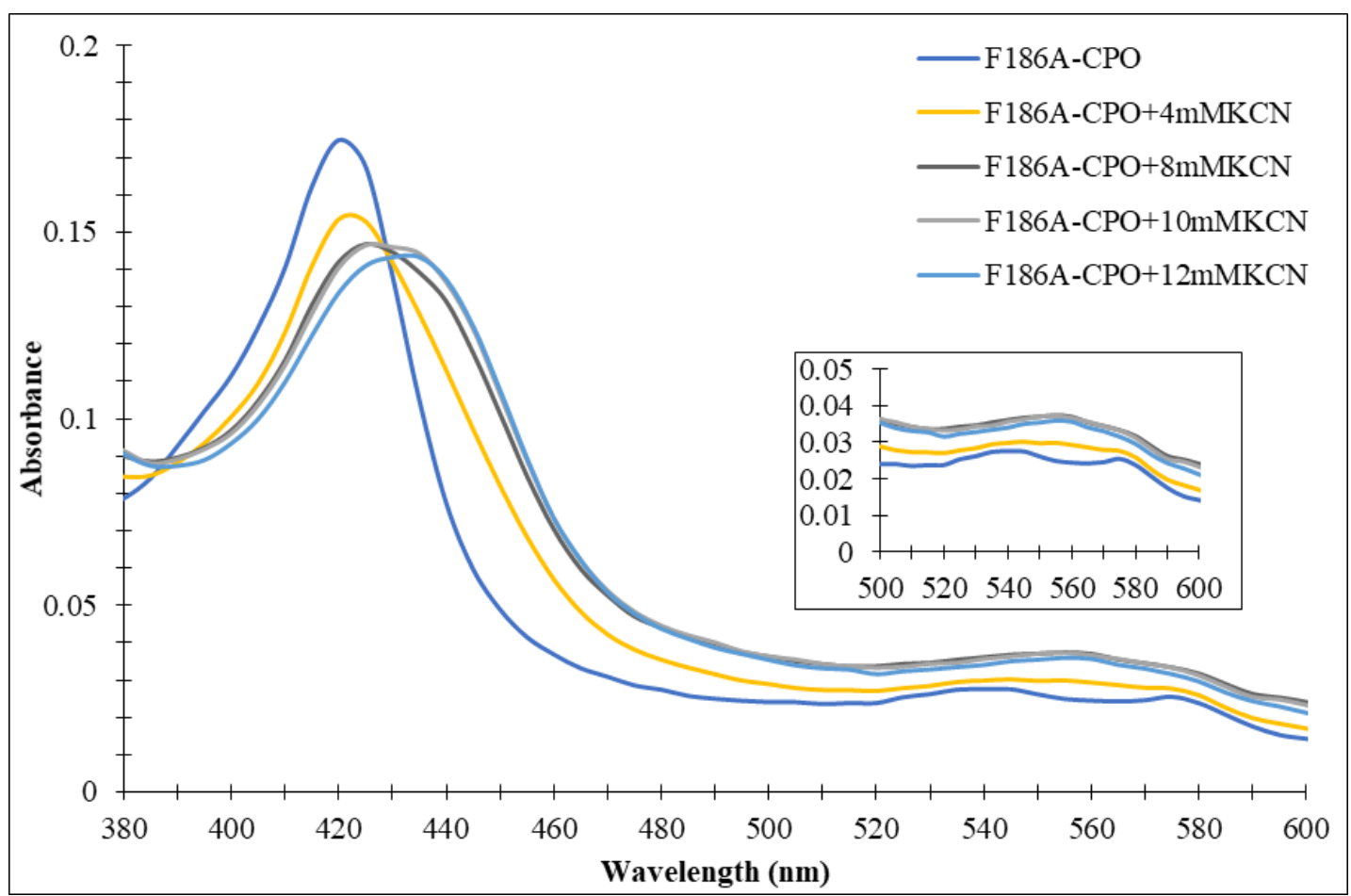

Figure 4.8 Titration of cyanide onto F186A-CPO in $25 \mathrm{mM}$ phosphate buffer pH 5.90. 


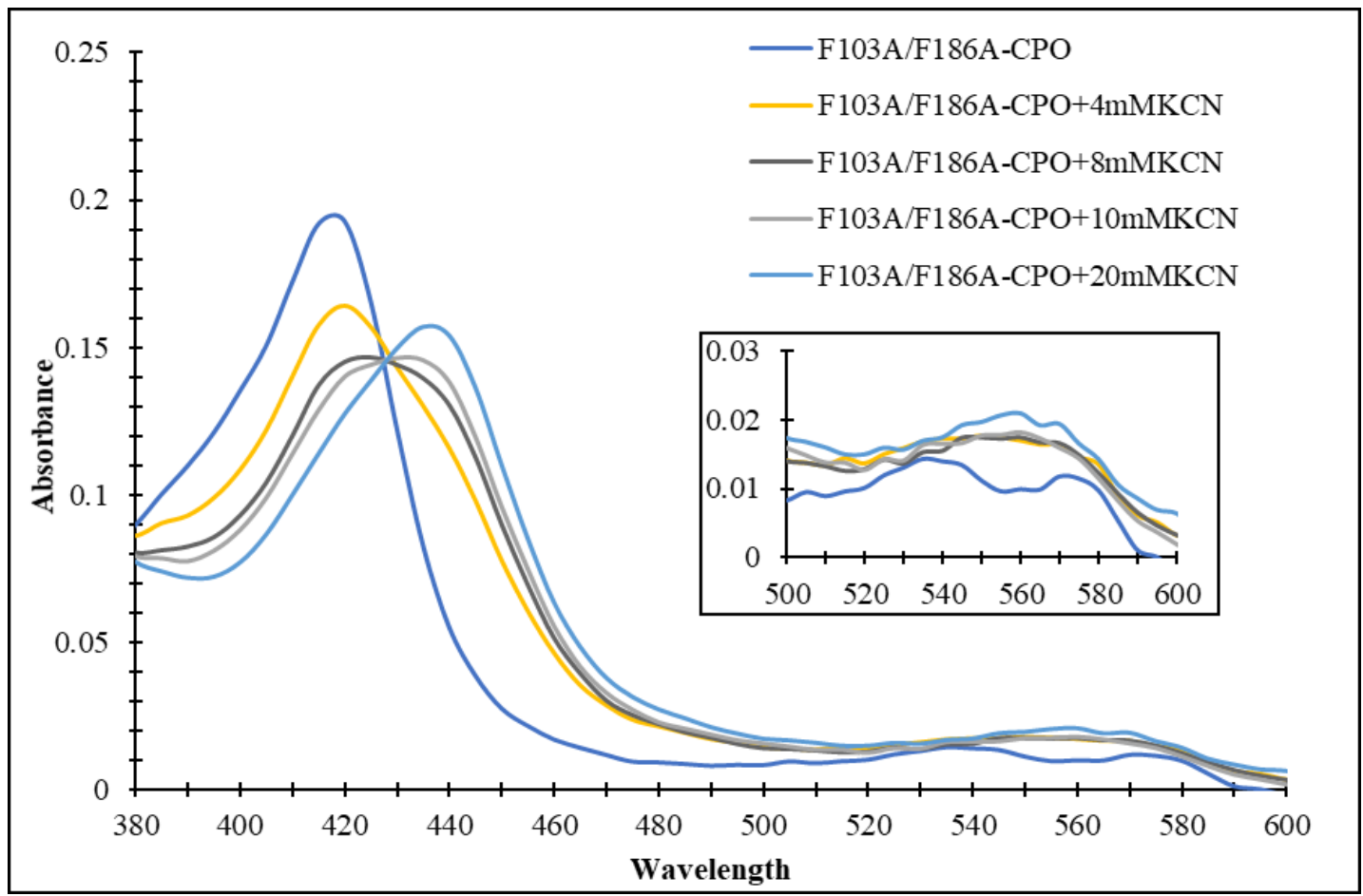

Figure 4.9 Titration of cyanide onto F103A/F186A-CPO in $25 \mathrm{mM}$ phosphate buffer pH 5.90.

\subsubsection{Catalytic Properties of WT-, F186A-, and F103A/F186A-CPO}

The changes introduced in the substrate-binding pocket should influence the catalytic properties of $\mathrm{CPO}$ as these residues interact with the incoming substrate and guarding their access to the heme center. In this part of my study, the $\mathrm{pH}$ profile of halogenation, peroxidation, epoxidation, and dismutation activities of the CPO mutants were tested and compared to that of WT-CPO.

\subsubsection{Halogenation Activity of WT-, F186A-, and F103A/F186A-CPO}

Chlorination is the primary function of CPO and this activity defined the enzyme as many haloperoxidases are incapable of catalyzing chlorinating reactions. The results of the mutants were quite interesting as a small single mutation in the substrate-binding pocket hindered the halogenation activity. In Figure 4.10, the optimum $\mathrm{pH}$ for chlorination 
catalyzed by WT-CPO is at 2.75 and the activity decreases significantly as the $\mathrm{pH}$ risen. However, F186A- and F103A/F186A-CPO shown no significant sign of chlorinating activity at the tested $\mathrm{pH}$ range. Similar results were observed for the bromination assay (Figure 4.11). The mutation in the substrate-binding pocket might possibly induced a slight, as there is no significant difference in the secondary structure, change in the halide-binding sites or induced a disruption of the proximity hydrogen bond between His105 and Glu183, which resulted in the demolition of CPO's halogenating activity. An alternate explanation is the mutation caused a decrease in the redox potential of Compound I where the oxidation of the halide ions is not permitted.

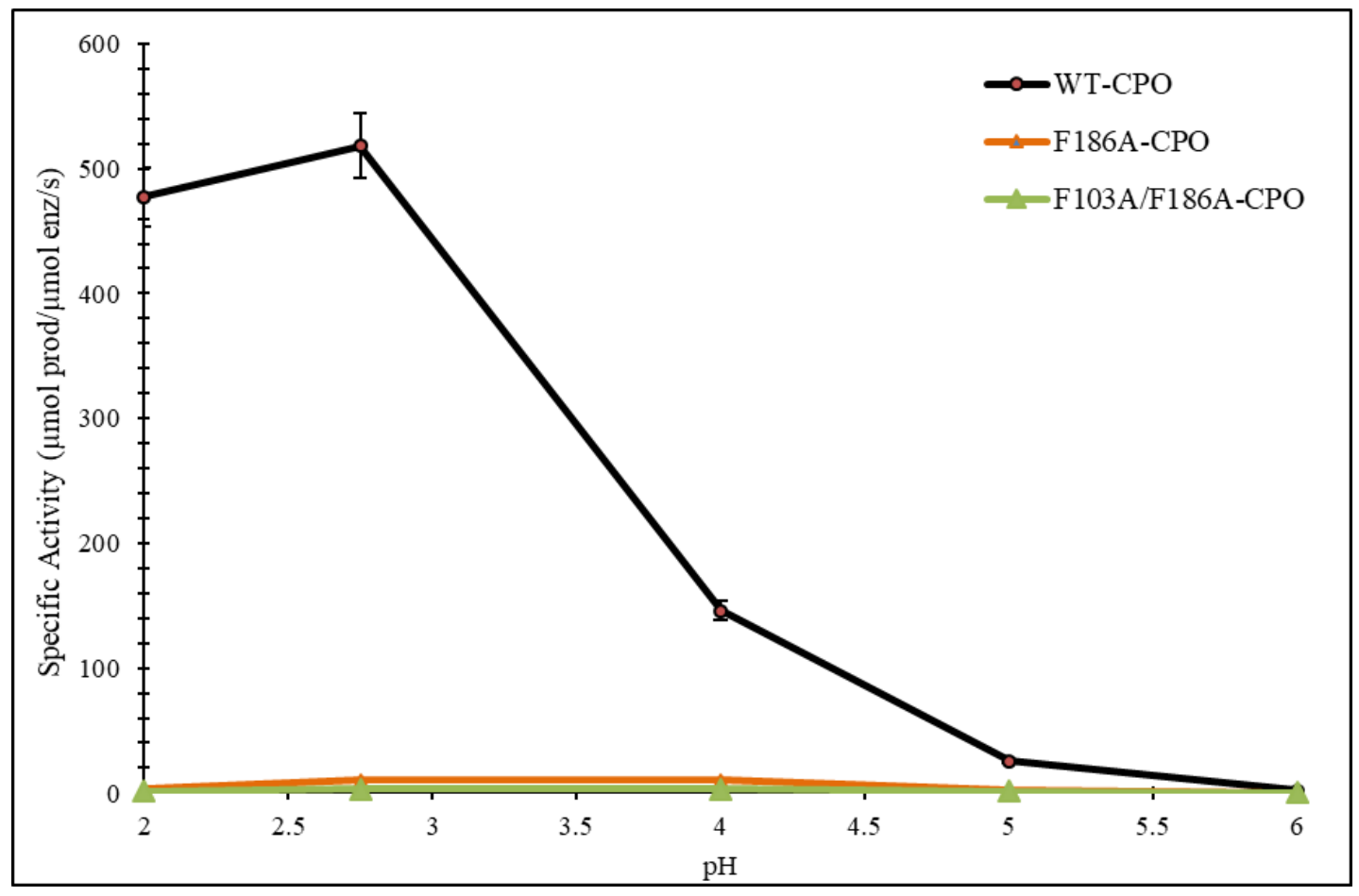

Figure 4.10 Specific activity of WT-, F186A- and F103A/F186A-CPO for chlorination of MCD assay at pH 2.00-6.00. (Some error bars are not visible on the graph). 


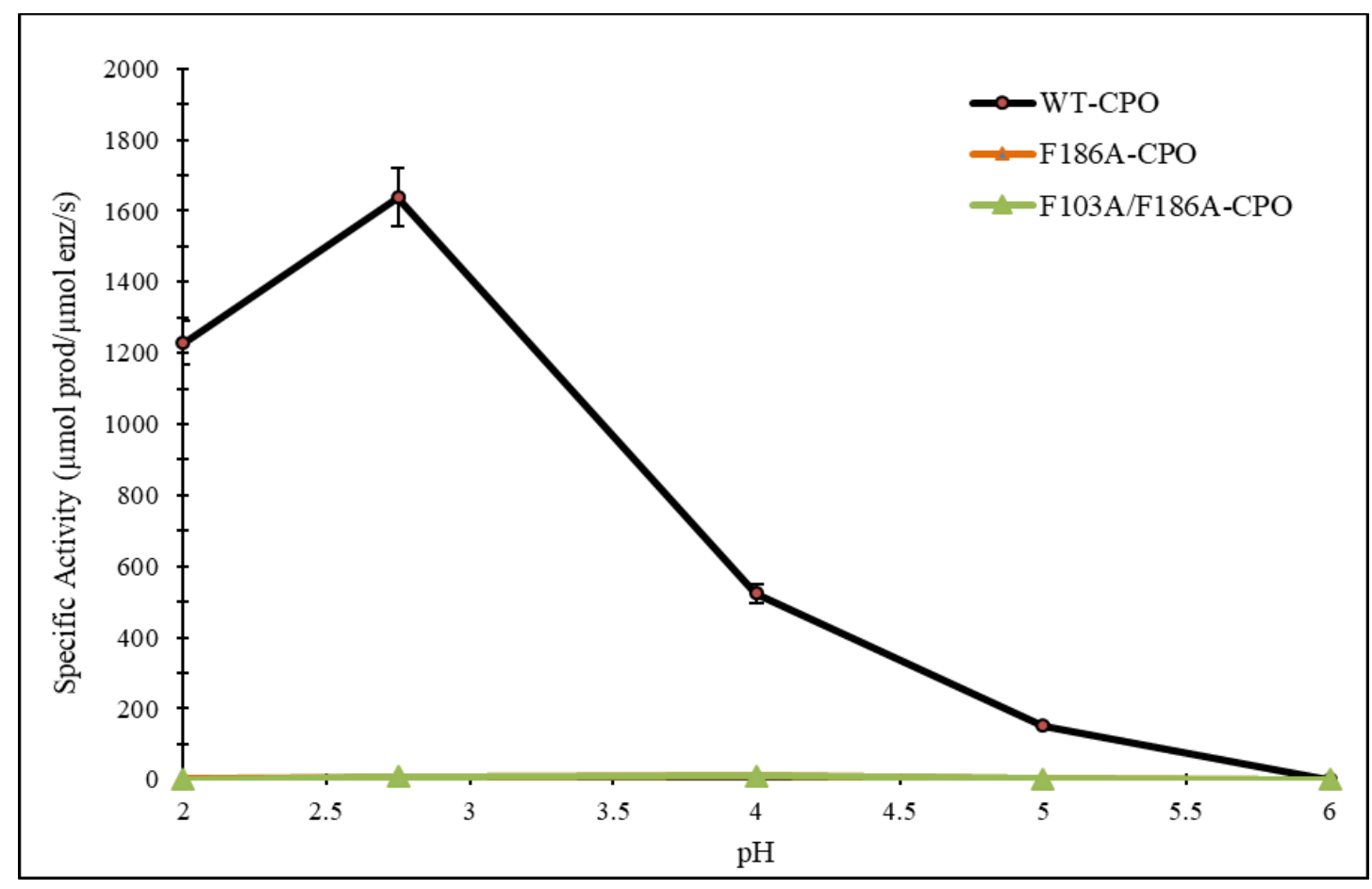

Figure 4.11 Specific activity of WT-, F186A- and F103A/F186A-CPO for bromination of MCD assay at pH 2.00-6.00. (Some error bars are not visible on the graph).

\subsubsection{Peroxidation Activity of WT-, F186A-, and F103A/F186A-CPO}

As a heme peroxidase, CPO can catalyze one electron oxidation of substrates yielding a radical product. In the case of ABTS assay, the substrate, ABTS, is oxidized in two one electron transfer process to $\mathrm{ABTS}^{2+}$. WT-CPO has an optimum $\mathrm{pH}$ of 2.50 for catalyzing peroxidation reaction and the activity is significantly reduced at $\mathrm{pH} 4.00$ or above (Figure 4.12). As for F186A- and F103A/F186A-CPO, the optimum $\mathrm{pH}$ is at 3.00 while the activity remained active at $\mathrm{pH} 4.00-5.00$ but the activity is negligible at $\mathrm{pH} 6.00$.

As observed in Figure 4.13, the comparison between WT-CPO and the CPO mutants at $\mathrm{pH} 2.50$ and 4.00 clearly demonstrated their difference. At pH 2.50, F186A- and F103A/F186A-CPO possess about 30\% activity relative to WT-CPO. However, the peroxidation activity of F186A- and F103A/F186A-CPO was enhanced by more than 8- 
folds relative to WT-CPO at $\mathrm{pH}$ 4.00. A plausible explanation for the difference in activity is the $\mathrm{pK}_{\mathrm{a}}$ of Compound II for the CPO mutants is greater than that of WT-CPO. The peroxidation pathway of CPO involved a second intermediate, Compound II, and the active state of this species was proposed to be the protonated form. Therefore, regulation of the $\mathrm{pK}_{\mathrm{a}}$ of Compound II plays an important role in the peroxidation activity and this could be the plausible explanation on the activity of the $\mathrm{CPO}$ mutants at higher $\mathrm{pH}$.

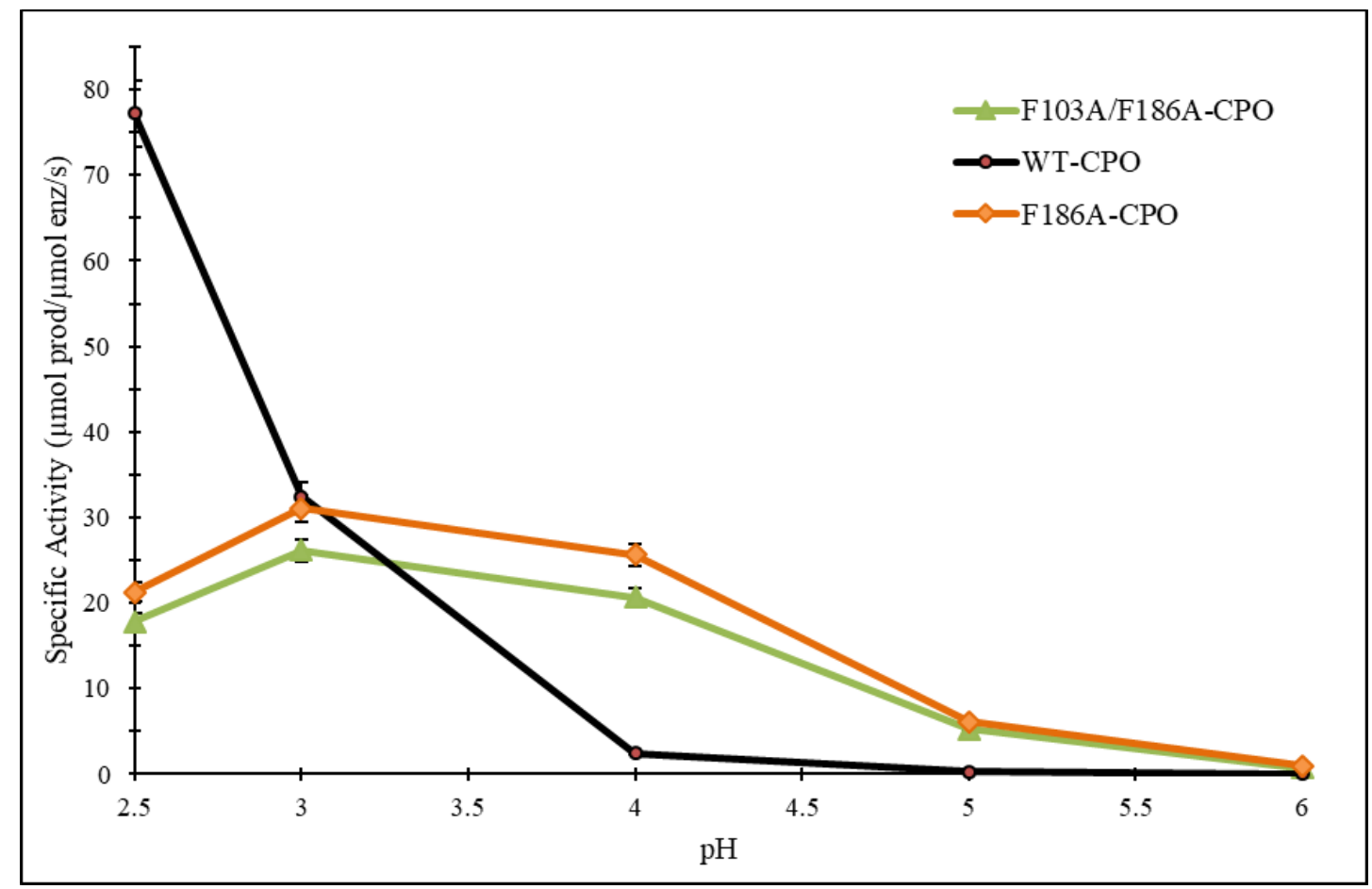

Figure 4.12 Specific activity of WT-, F186A- and F103A/F186A-CPO for peroxidation of ABTS assay at pH 2.50-6.00. (Some error bars are not visible on the graph). 


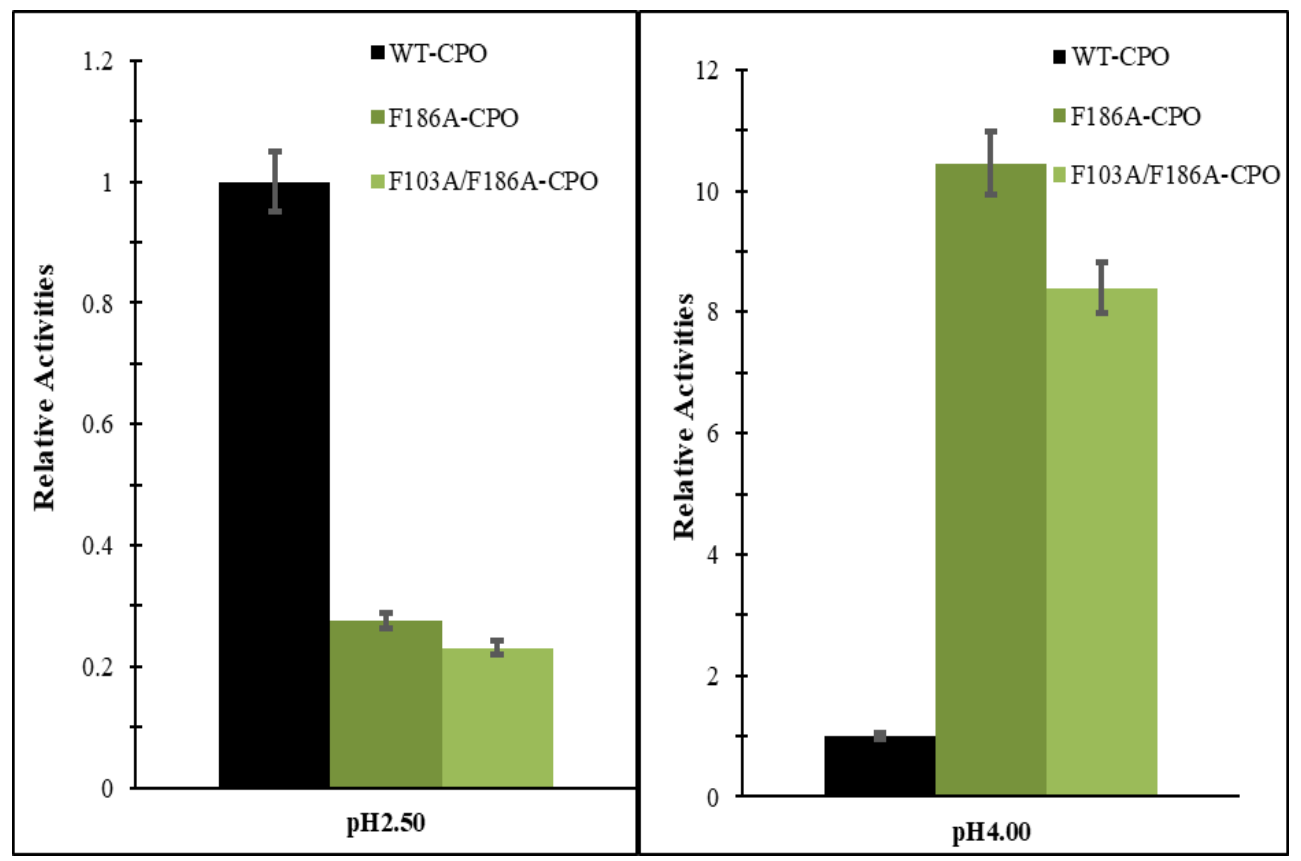

Figure 4.13 Relative activity of WT-, F186A- and F103A/F186A-CPO for peroxidation of ABTS assay at pH 2.50 (Left) and 4.00 (Right). (Some error bars are not visible on the graph).

\subsubsection{Epoxidation and Dismutation Activity of WT-, F186A-, and F103A/F186A- CPO}

The epoxidation activity of WT-, F186A-, and F103A/F186A-CPO was evaluated at $\mathrm{pH}$ 3.00-5.50. Within this $\mathrm{pH}$ range, WT-CPO and the mutants displayed a similar trend as observed in Figure 4.14. Even though they shared a similar trend, the relative activity differed. In Figure 4.15, the activity of F186A- and F103A/F186A-CPO is relatively 7- and 8-folds of WT-CPO at $\mathrm{pH} 4.50$, respectively. 


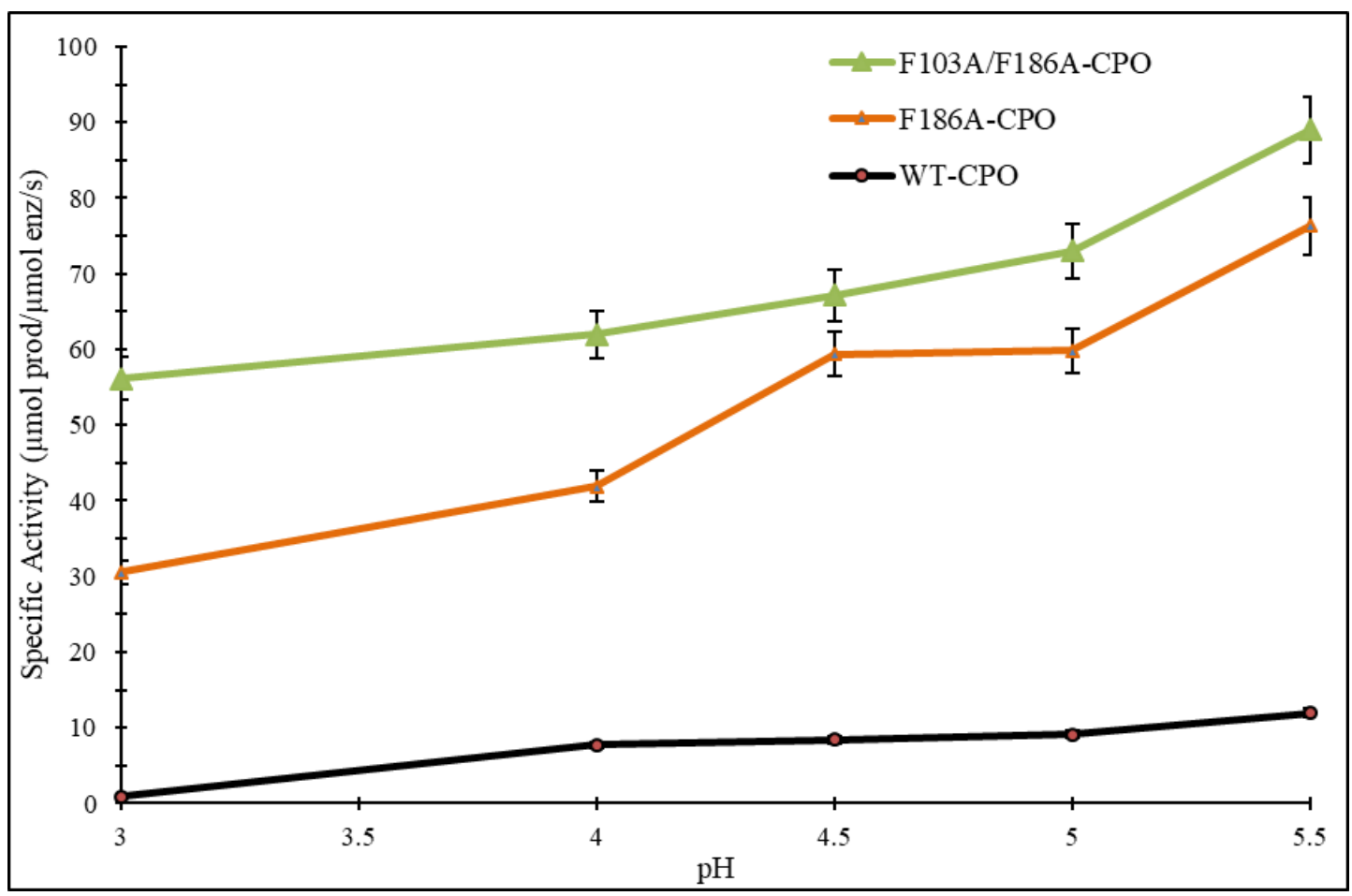

Figure 4.14 Specific activity of WT-, F186A- and F103A/F186A-CPO for epoxidation of styrene assay at pH 3.00-5.50. (Some error bars are not visible on the graph).

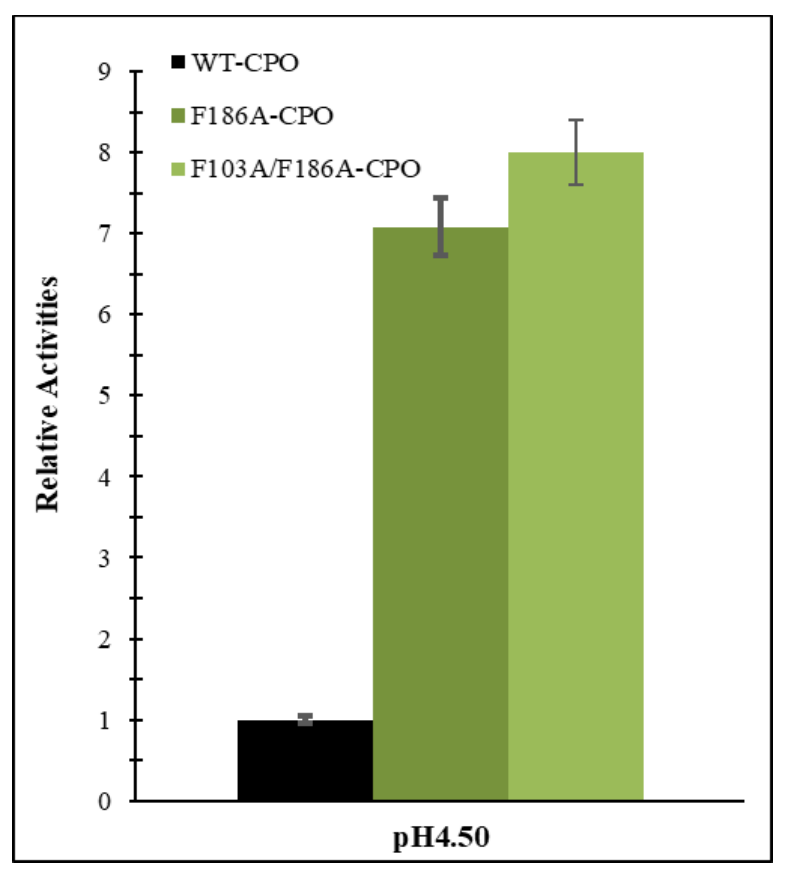

Figure 4.15 Relative activity of WT-, F186A- and F103A/F186A-CPO for epoxidation of styrene assay at pH 4.50. 
This significant enhancement might be at the expense of the dismutation pathway as they have a competing relationship. In Figure 4.16, the catalatic activity of F186A- and F103A/F186A-CPO was significantly less than that of WT-CPO at the $\mathrm{pH}$ profile of 2.506.00. The decrease in the catalatic activity of CPO allowed more oxidants to be utilized in catalyzing the epoxidation of styrene as enhancement was observed for the mutants. As proposed in the previous sections, the plausible reduction on the redox potential of the CPO mutants' Compound I might be the cause on the changes in the dismutation activity, as the oxidation of hydrogen peroxide is prohibited.

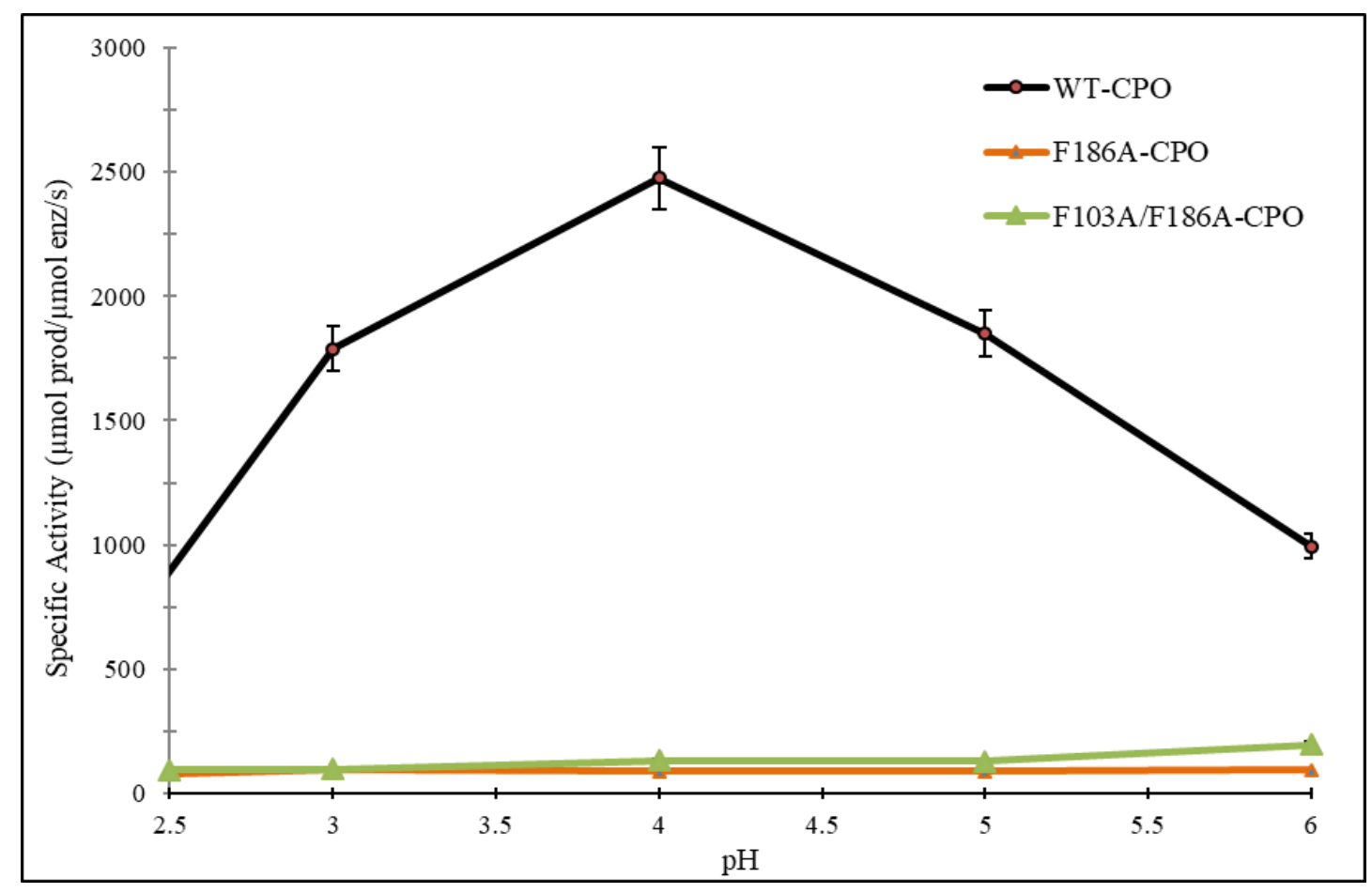

Figure 4.16 Specific activity of WT-, F186A-, and F103A/F186A-CPO for dismutation of hydrogen peroxide assay at pH 2.50-6.00. (Some error bars are not visible on the graph). 


\subsubsection{Epoxidation of Styrene and its derivatives catalyzed by WT-, F186A-, and F103A/F186A-CPO}

CPO catalyzes the epoxidation of styrene and its derivatives yielding epoxides in $(R)$ and $(S)$-configurations. These enantiomers were proven to be successfully separated and identified by Whelk-O 1 column of HPLC. It has been previously identified that Whelk-O 1 column can maintain the same elution order for similar enantiomers [139]. The elution order of the column tends to be the $(R)$-enantiomer then followed by the $(S)$-enantiomer as the retention time of the $(R)$-configuration is shorter than that of the $(S)$-configuration in Whelk-O 1 column. This elution order of the enantiomers was confirmed previously in our lab through the $(R)$ - and $(S)$-styrene oxide standards [140].

\subsubsection{Epoxidation of Styrene}

Th products of the styrene epoxidation catalyzed by WT-, F186A-, and F103A/F186ACPO were extracted and analyzed by HPLC. As observed in Figure 4.17, 4.18, and 4.19, WT-CPO and the CPO mutants yielded two products, $(R)$ - and $(S)$-styrene oxide. The resolution factor, $\mathrm{R}_{\mathrm{s}}$, of WT-, F186A-, and F103A/F186A-CPO were 2.27, 1.67, and 1.82, respectively. The retention factors, $\mathrm{k}_{1}$ ' and $\mathrm{k}_{2}$, for WT-CPO were 6.04 and 7.71, respectively. As for the mutants, F186A-CPO's retention factors were 6.12 and 7.78 and F103A/F186A-CPO's retention factors were 6.42 and 8.17 . The separation factors, $\alpha$, of WT-, F186A-, and F103A/F186A-CPO were fairly similar as they were 1.28, 1.27, and 1.27 , respectively. 


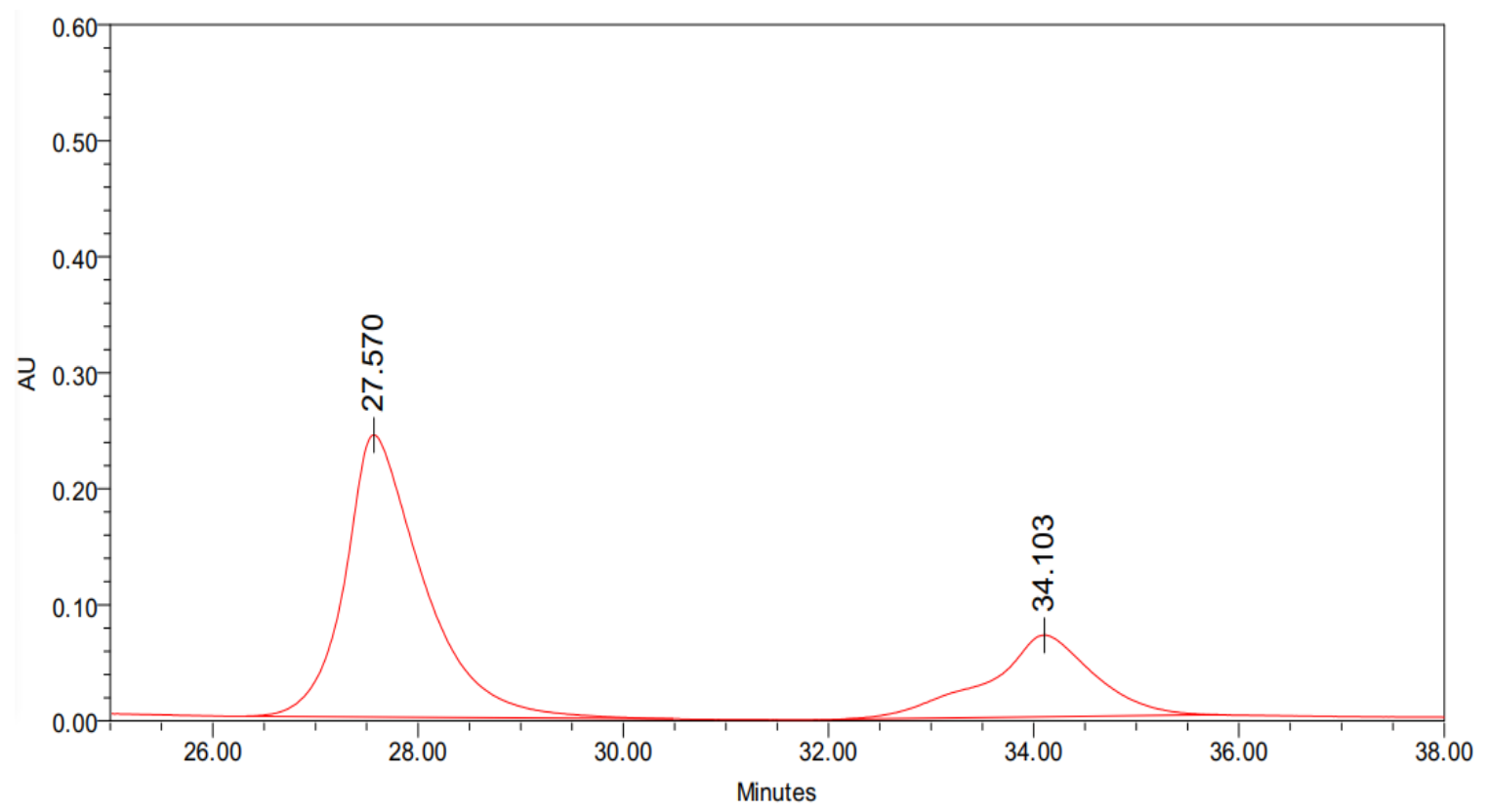

Figure 4.17 Enantioseparation of styrene oxide catalyzed by WT-CPO on Whelk-O 1 column at room temperature with pure hexane as mobile phase while the absorbance is monitored at $215.7 \mathrm{~nm}$.

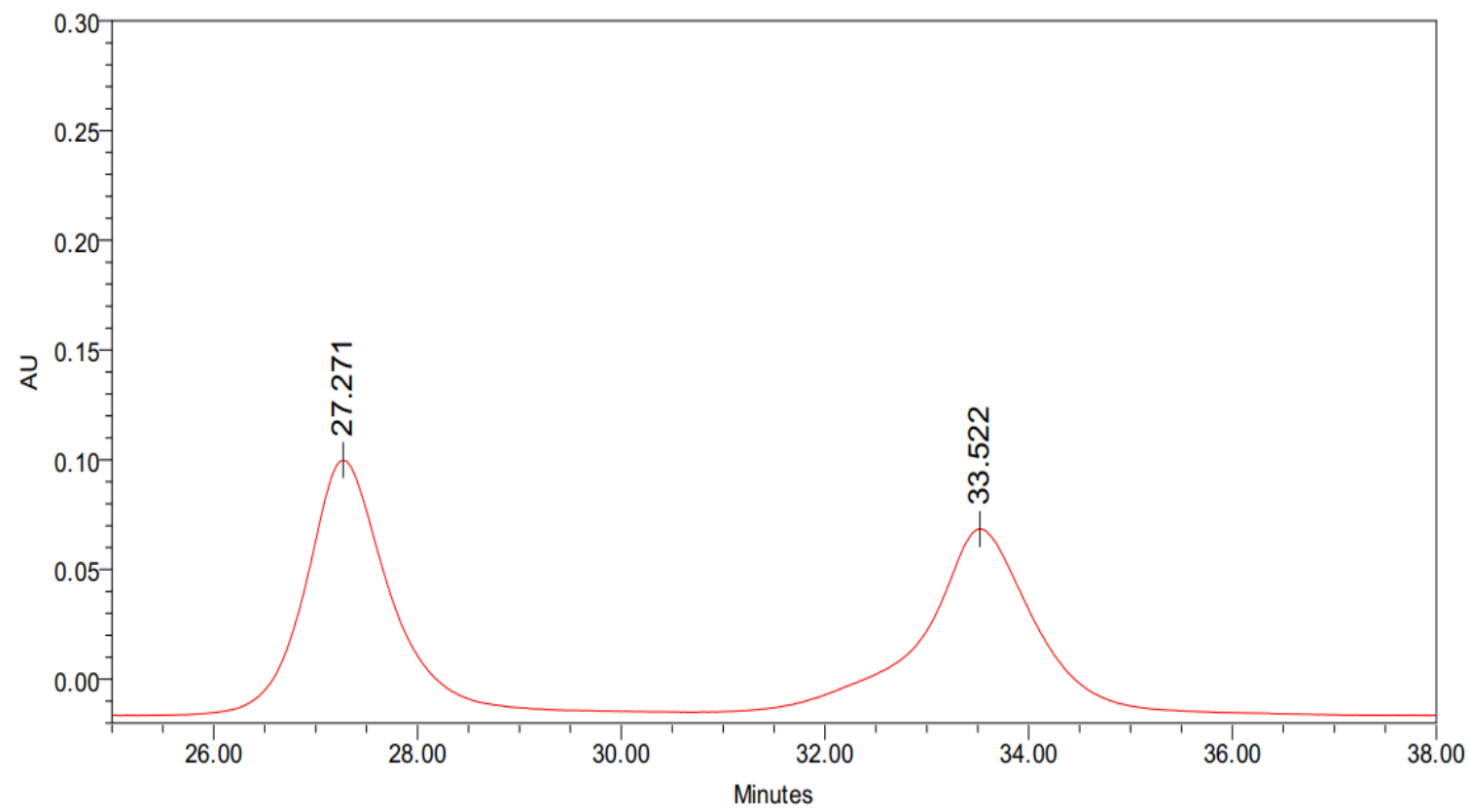

Figure 4.18 Enantioseparation of styrene oxide catalyzed by F186A-CPO on Whelk$O 1$ column at room temperature with pure hexane as mobile phase while the absorbance is monitored at $215.7 \mathrm{~nm}$. 


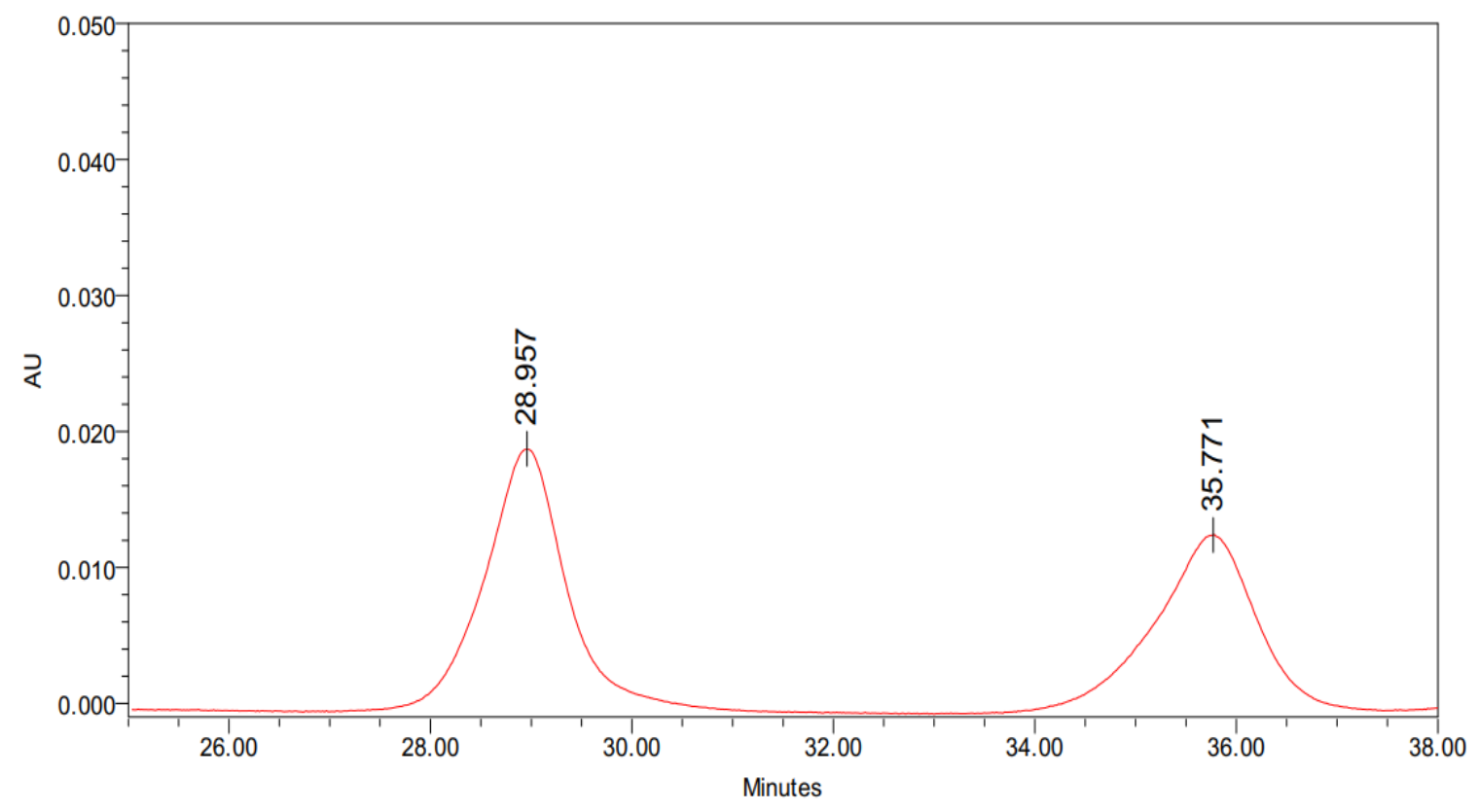

Figure 4.19 Enantioseparation of styrene oxide catalyzed by F103A/F186A-CPO on Whelk-O 1 column at room temperature with pure hexane as mobile phase while the absorbance is monitored at $215.7 \mathrm{~nm}$.

The epoxidation of styrene catalyzed by WT-CPO yielded $(R)$-styrene oxide as major product with an enantiomeric excess of 44\%. As for F186A- and F103A/F186A-CPO, the ee value was $3 \%$ and $9 \%$, respectively. The significant decrease on the ee value for the mutants suggested that the original interactions between styrene and Phe103/Phe186 were disrupted. The larger substrate-binding pocket in the mutants allowed styrene to bind in more conformations thus lead to the decrease on the enantioselectivity.

\subsubsection{Epoxidation of cis- $\beta$-methylstyrene}

CPO catalyzes the epoxidation of cis- $\beta$-methylstyrene yielding only $(1 S, 2 R)$-cis- $\beta$ methylstyrene oxide as observed in Figure 4.20. However, F186A- and F103A/F186ACPO yielded two products, one has been previously identified as the $(1 S, 2 R)$-enantiomer (Figure 4.21 and 4.22). The separation factor for the two mutants was the same, 1.06. As for the retention factors, F186A-CPO were 5.79 and 6.12 while F103A/F186A-CPO were 
6.02 and 6.38. The calculated $\mathrm{R}_{\mathrm{s}}$ for F186A- and F103A/F186A-CPO was 0.78 and 0.73 , respectively. The reaction catalyzed by F186A-CPO yielded an ee value of $42 \%$ while F103A/F186A-CPO only yielded an ee value 12\%. The mutations on Phe103 and Phe186 disturbed the Phe103/Phe186 clamp and their interactions with the substrate, which result in the significant loss on the enantioselectivity for cis- $\beta$-methylstyrene. The enantioselectivity decreased more significantly as the size of the substrate-binding pocket increased.

\subsubsection{Epoxidation of trans- $\beta$-methylstyrene}

The epoxidation of trans- $\beta$-methylstyrene catalyzed by WT-CPO and the mutants yielded $(1 R, 2 R)$-enantiomer and $(1 S, 2 S)$-enantiomer in the same elution order as illustrated in the Figure 4.23, 4.24 and 4.25. The presence of a third peak in the spectrum could be the further oxidized or hydrolyzed product of the reaction. The calculated resolution factors were 3.90, 4.09, and 4.96 for WT-, F186A-, and F103A/F186A-CPO, respectively. The retention factors, $\mathrm{k}_{1}$ ' and k2', for WT-CPO were 7.70 and 12.10. As for the mutants, F186A-CPO's retention factors were 7.92 and 12.52 and F103A/F186ACPO's retention factors were 7.75 and 12.37 . The ee values for WT-CPO was only $8 \%$ while F186A-CPO was 19\%. The double mutant, F103A/F186A-CPO, has an ee value of 55\%. Since the interactions between WT-CPO and trans- $\beta$-methylstyrene restricted the access to the heme center, the presence of a larger substrate-binding pocket in the CPO mutants can better accommodate the substrate, thus enhanced the yield and enantioselectivity for this reaction. 


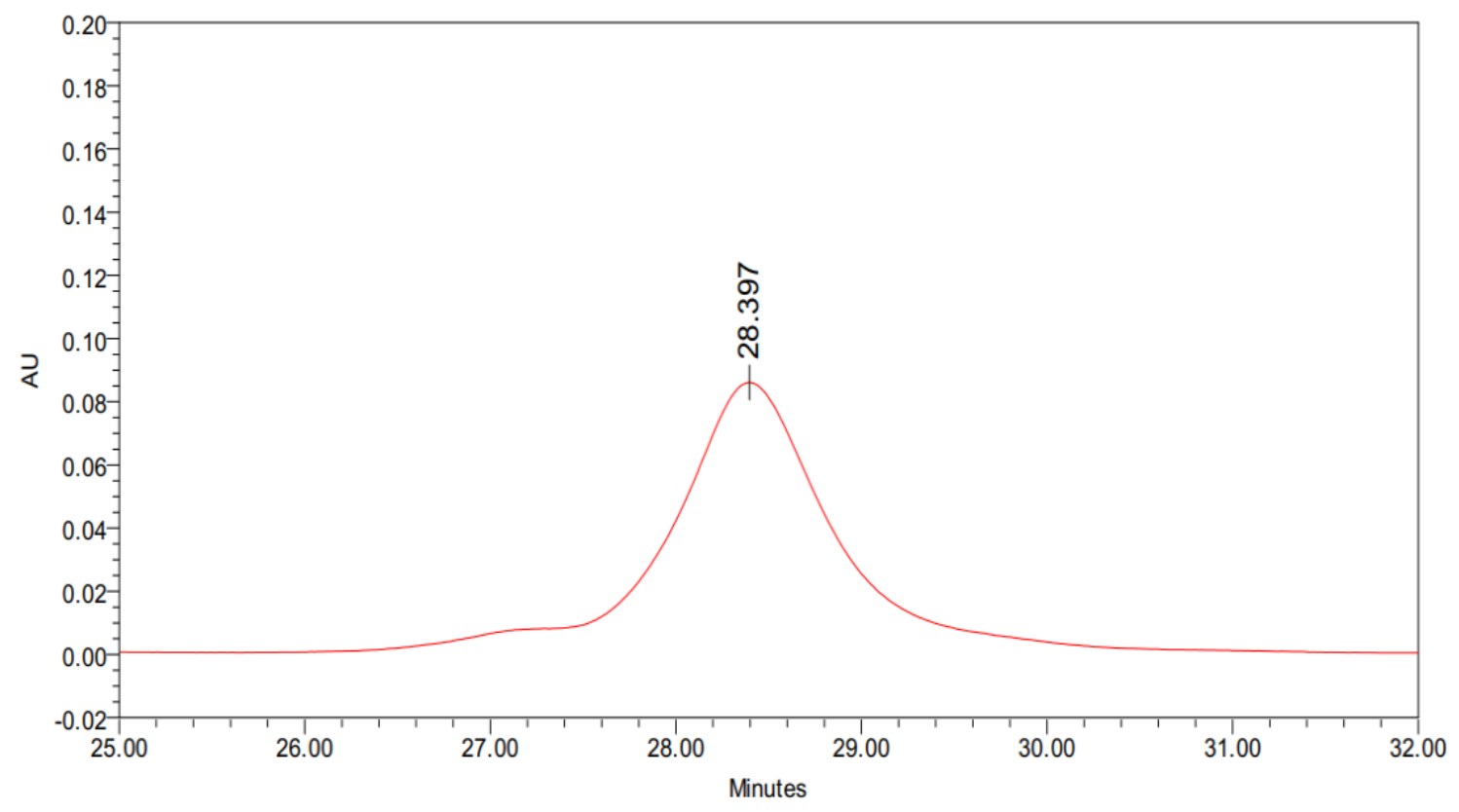

Figure 4.20 Enantioseparation of cis- $\beta$-methylstyrene oxide catalyzed by WT-CPO on Whelk-O 1 column at room temperature with pure hexane as mobile phase while the absorbance is monitored at $215.7 \mathrm{~nm}$.

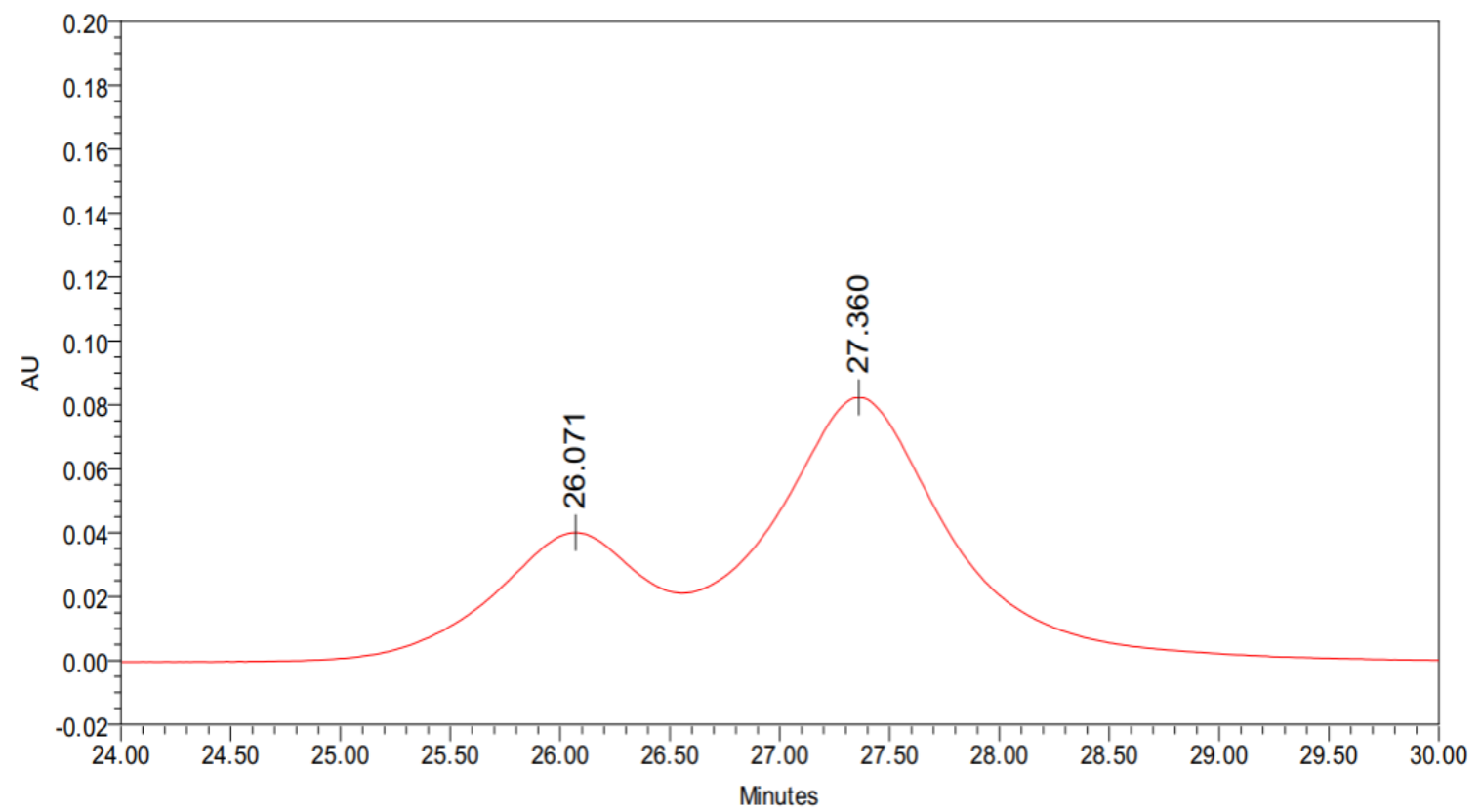

Figure 4.21 Enantioseparation of cis- $\beta$-methylstyrene oxide catalyzed by F186A-CPO on Whelk-O 1 column at room temperature with pure hexane as mobile phase while the absorbance is monitored at $215.7 \mathrm{~nm}$. 


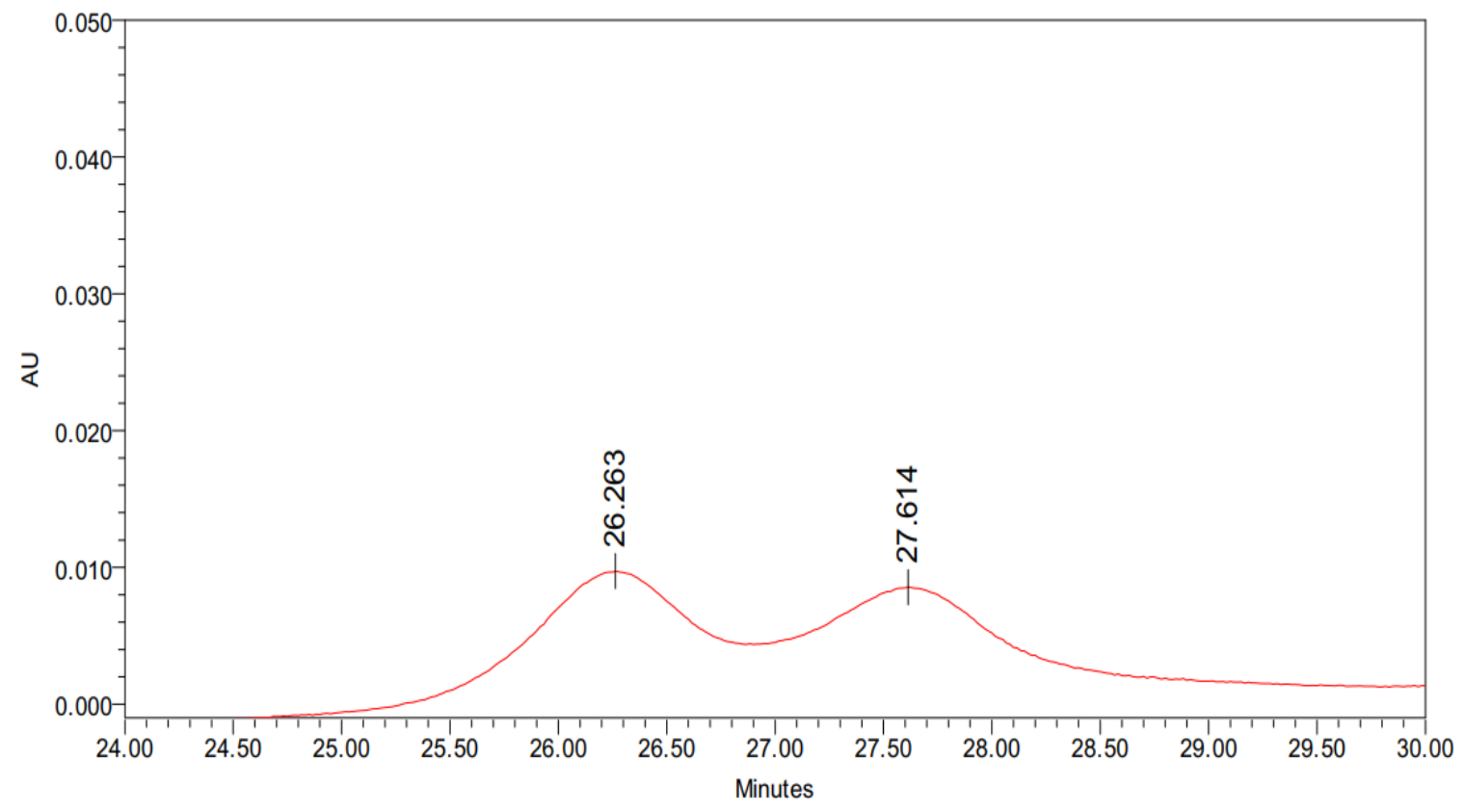

Figure 4.22 Enantioseparation of cis- $\beta$-methylstyrene oxide catalyzed by F103AF186A-CPO on Whelk-O 1 column at room temperature with pure hexane as mobile phase while the absorbance is monitored at $215.7 \mathrm{~nm}$.

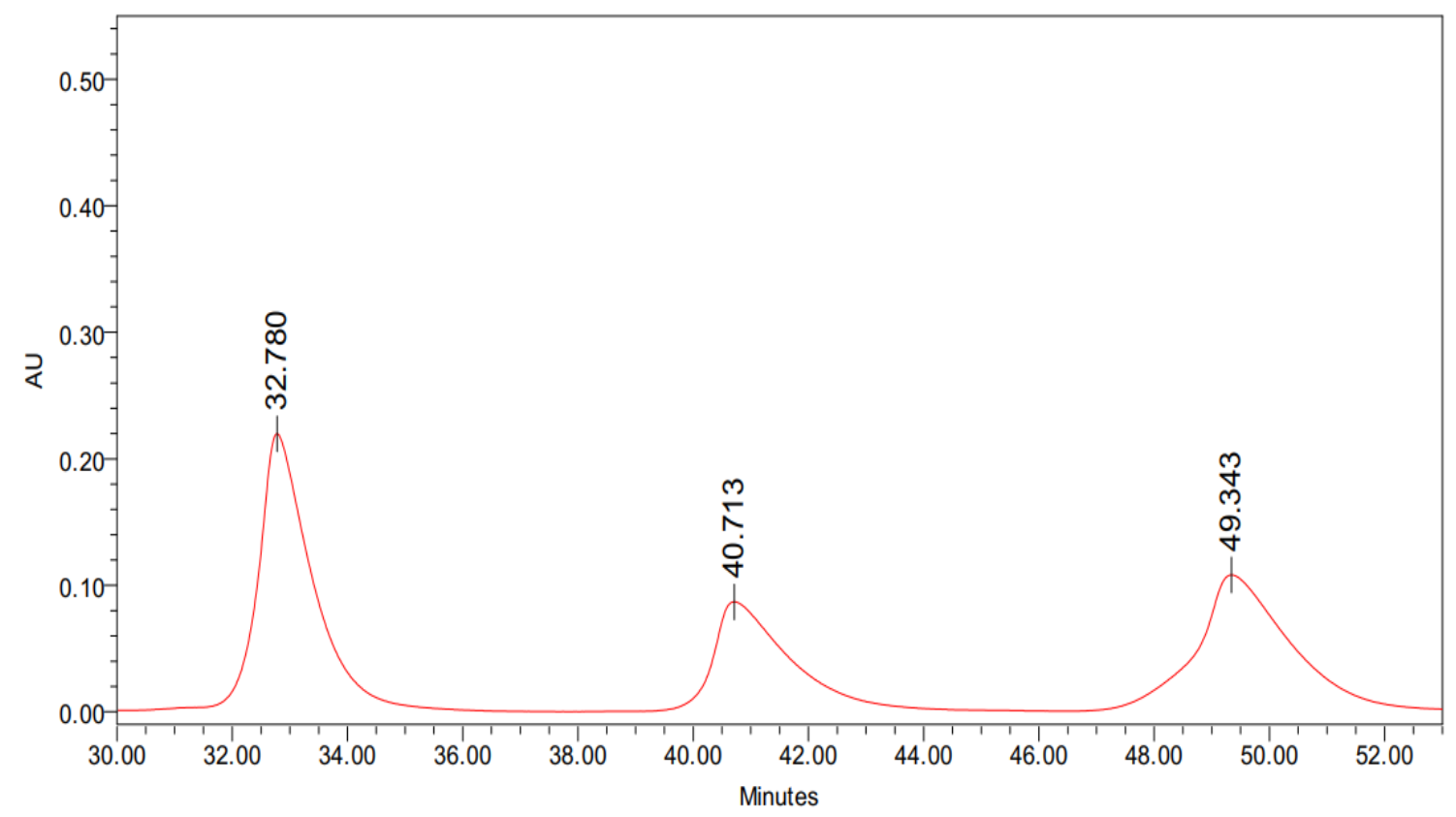

Figure 4.23 Enantioseparation of trans- $\beta$-methylstyrene oxide catalyzed by WT-CPO on Whelk-O 1 column at room temperature with pure hexane as mobile phase while the absorbance is monitored at $215.7 \mathrm{~nm}$. 


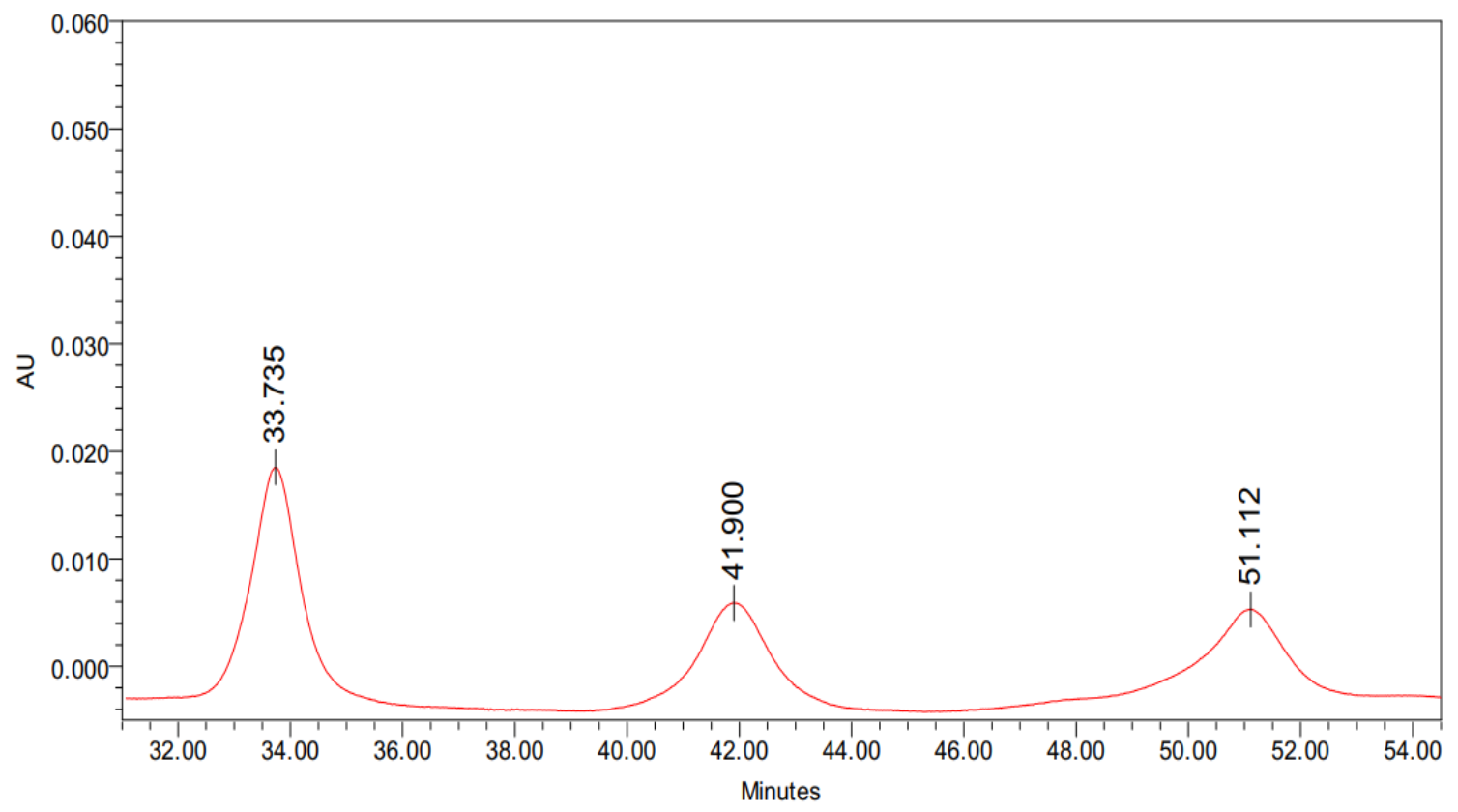

Figure 4.24 Enantioseparation of trans- $\beta$-methylstyrene oxide catalyzed by F186ACPO on Whelk-O 1 column at room temperature with pure hexane as mobile phase while the absorbance is monitored at $215.7 \mathrm{~nm}$.

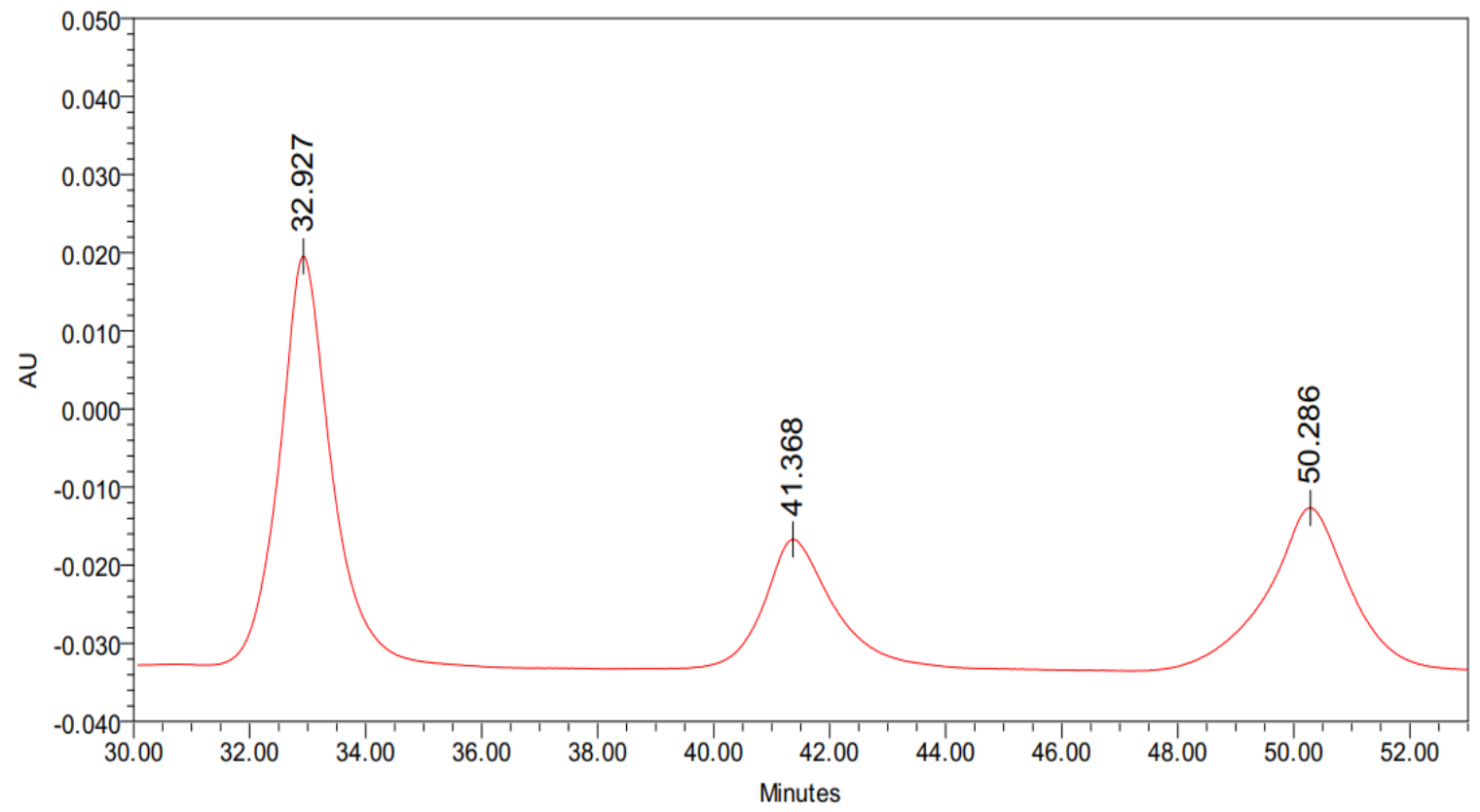

Figure 4.25 Enantioseparation of trans- $\beta$-methylstyrene oxide catalyzed by F103A/F186A-CPO on Whelk-O 1 column at room temperature with pure hexane as mobile phase while the absorbance is monitored at $215.7 \mathrm{~nm}$. 


\subsubsection{Epoxidation of trans- $\beta$-ethylstyrene}

In the epoxidation reaction of trans- $\beta$-ethylstyrene, WT- CPO and the CPO mutants yielded two products, the $(1 R, 2 R)$-enantiomer and the $(1 S, 2 S)$-enantiomer, as observed in Figure 4.26, 4.27, and 4.28. The retention factors were 5.52 and 8.12 for WT-CPO, 5.78 and 8.39 for F186A-CPO, and 5.96 and 8.61 for F103A/F186A-CPO. The separation factors of these products were 1.47, 1.45, and 1.44 for WT-, F186A-, and F103A/F186A$\mathrm{CPO}$, respectively. In comparison of the ee values, the CPO mutant displayed improvement to the enantioselectivity of the reaction as the ee value for WT-, F186A-, and F103A/F186A-CPO were 3\%, 23\%, and 21\%, respectively. Interestingly, the epoxidation of trans- $\beta$-ethylstyrene catalyzed by the two mutants favors the yield of the $(1 S, 2 S)$ enantiomer.

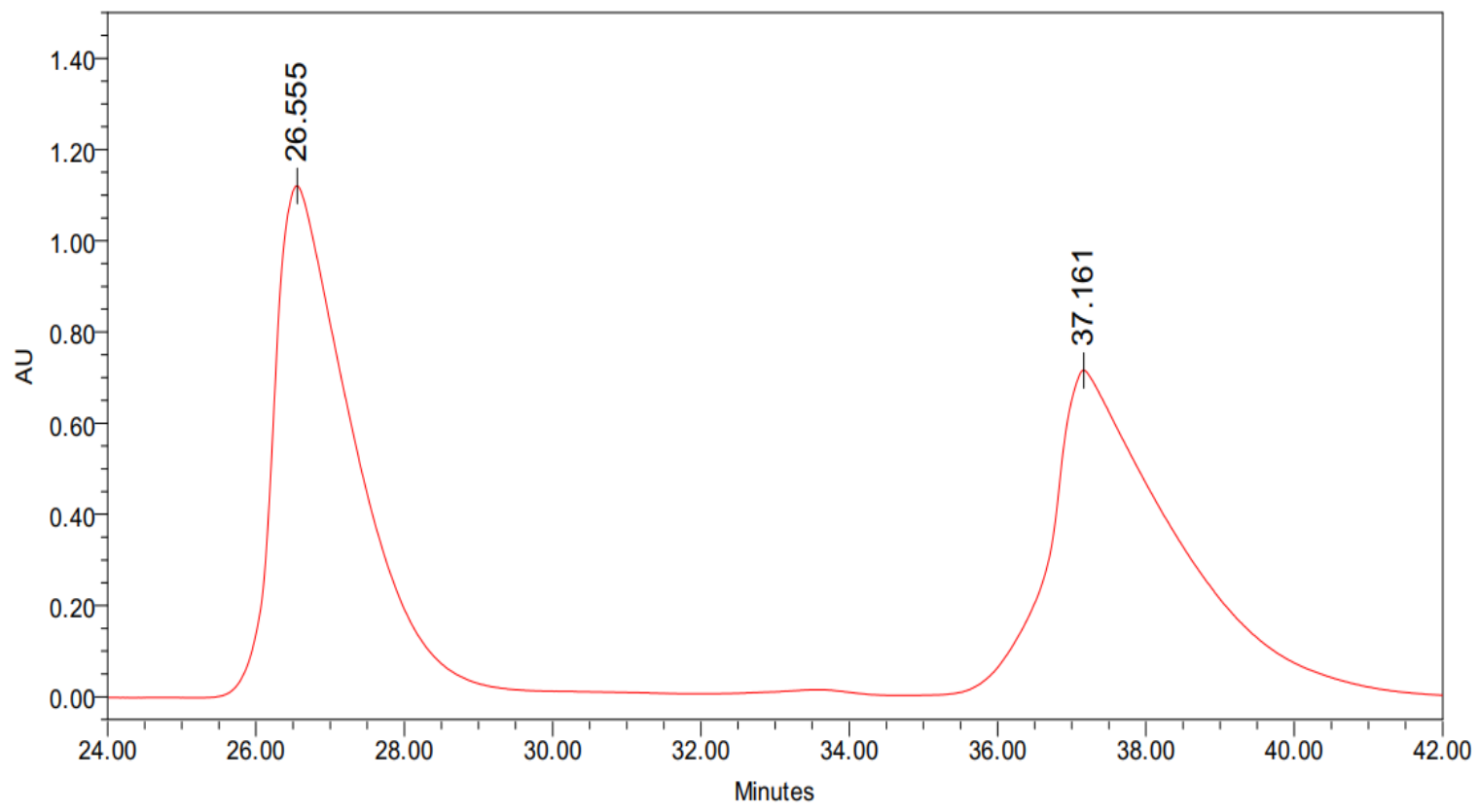

Figure 4.26 Enantioseparation of trans- $\beta$-ethylstyrene oxide catalyzed by WT-CPO on Whelk-O 1 column at room temperature with pure hexane as mobile phase while the absorbance is monitored at $218.0 \mathrm{~nm}$. 


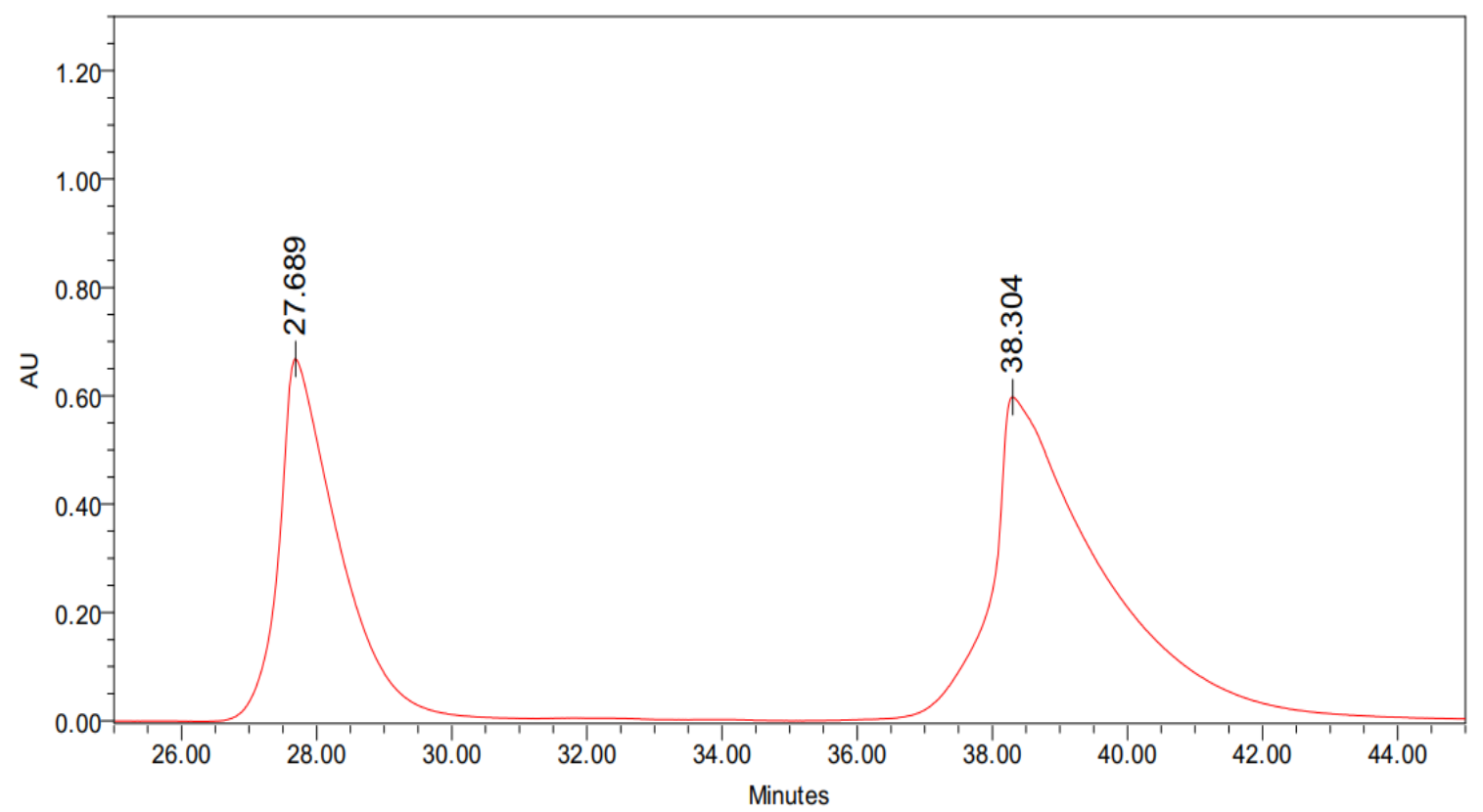

Figure 4.27 Enantioseparation of trans- $\beta$-ethylstyrene oxide catalyzed by F186A-CPO on Whelk-O 1 column at room temperature with pure hexane as mobile phase while the absorbance is monitored at $218.0 \mathrm{~nm}$.

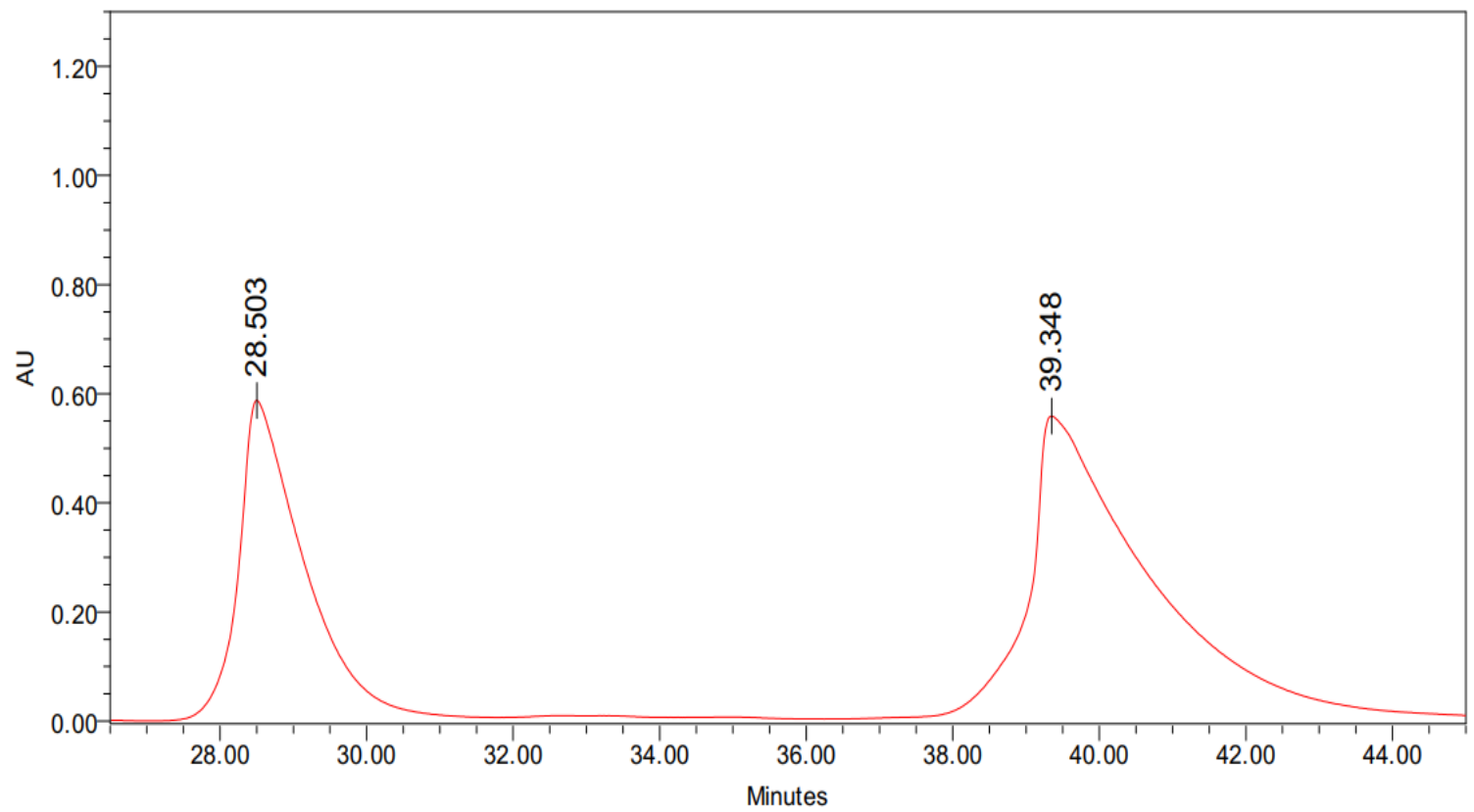

Figure 4.28 Enantioseparation of trans- $\beta$-ethylstyrene oxide catalyzed by F103A/F186A-CPO on Whelk-O 1 column at room temperature with pure hexane as mobile phase while the absorbance is monitored at $218.0 \mathrm{~nm}$. 


\subsubsection{Epoxidation of trans- $\beta$-propylstyrene}

The epoxidation of trans- $\beta$-propylstyrene catalyzed by WT-CPO and the mutants yielded $(R)$-enantiomer and $(S)$-enantiomer in the same elution order. In Figure 4.29, 4.30, and 4.31, the separation factors were calculated to be 1.45, 1.45, and 1.47 for WT-, F186A-, and F103A/F186A-CPO, respectively. The retention factors, $\mathrm{k}_{1}$ ' and $\mathrm{k}_{2}$, for WT-CPO were 6.70 and 9.73. As for the mutants, F186A-CPO's retention factors were 6.36 and 9.24 and F103A/F186A-CPO's retention factors were 5.76 and 8.44. As for the resolution factor, WT-, F186A-, and F103A/F186A-CPO were 3.16, 3.34, and 2.73, respectively. The ee values for the reaction catalyzed by WT-CPO was $11 \%$ while F186A-CPO was $10 \%$. The double mutant, F103A/F186A-CPO, has an ee value of 14\%. The minor difference between WT-, F186A-, and F103A/F186A-CPO clearly indicated that they have similar enantioselectivity for trans- $\beta$-propylstyrene.

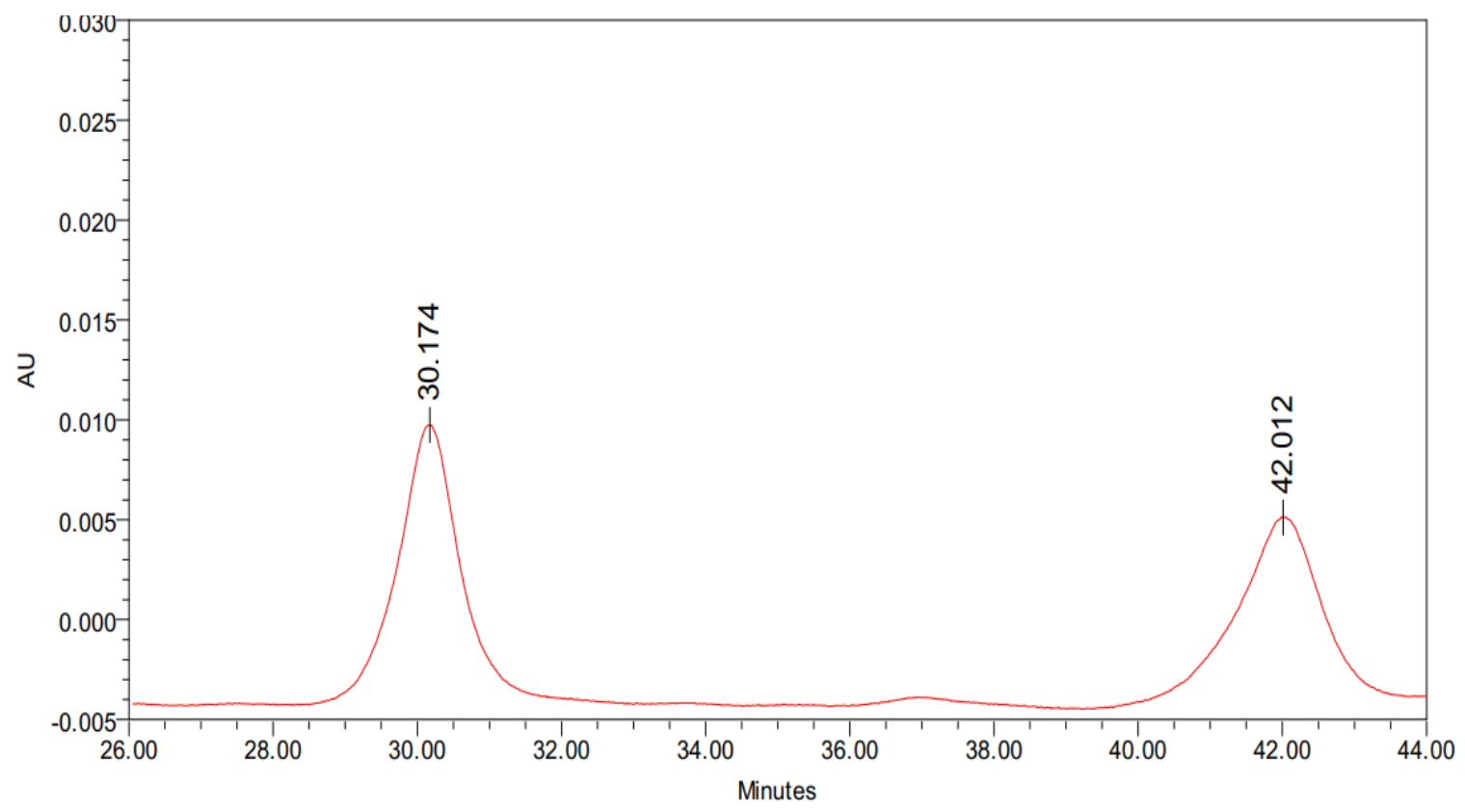

Figure 4.29 Enantioseparation of trans- $\beta$-propylstyrene oxide catalyzed by WT-CPO on Whelk-O 1 column at room temperature with pure hexane as mobile phase while the absorbance is monitored at $218.0 \mathrm{~nm}$. 


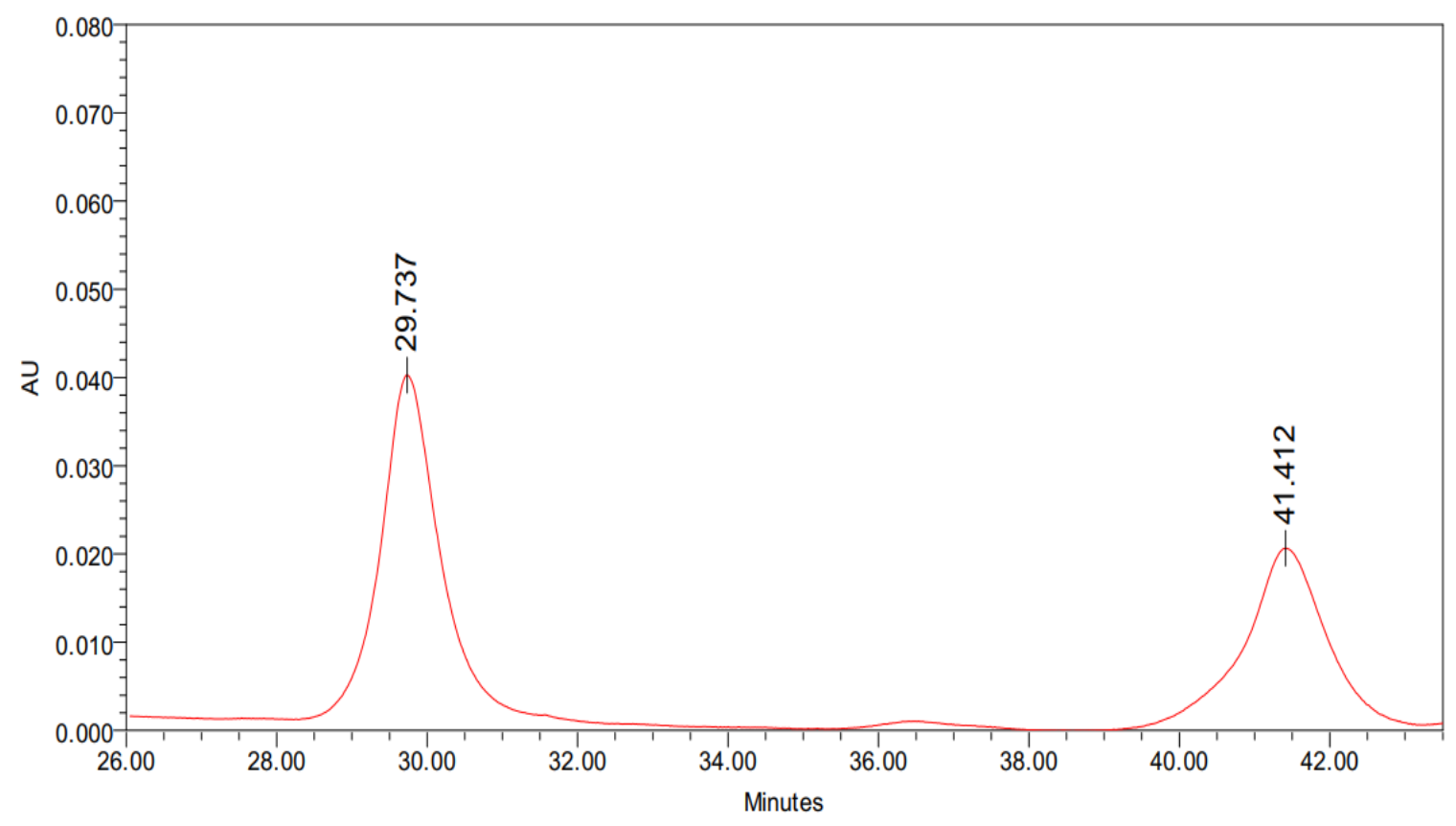

Figure 4.30 Enantioseparation of trans- $\beta$-propylstyrene oxide catalyzed by F186ACPO on Whelk-O 1 column at room temperature with pure hexane as mobile phase while the absorbance is monitored at $218.0 \mathrm{~nm}$.

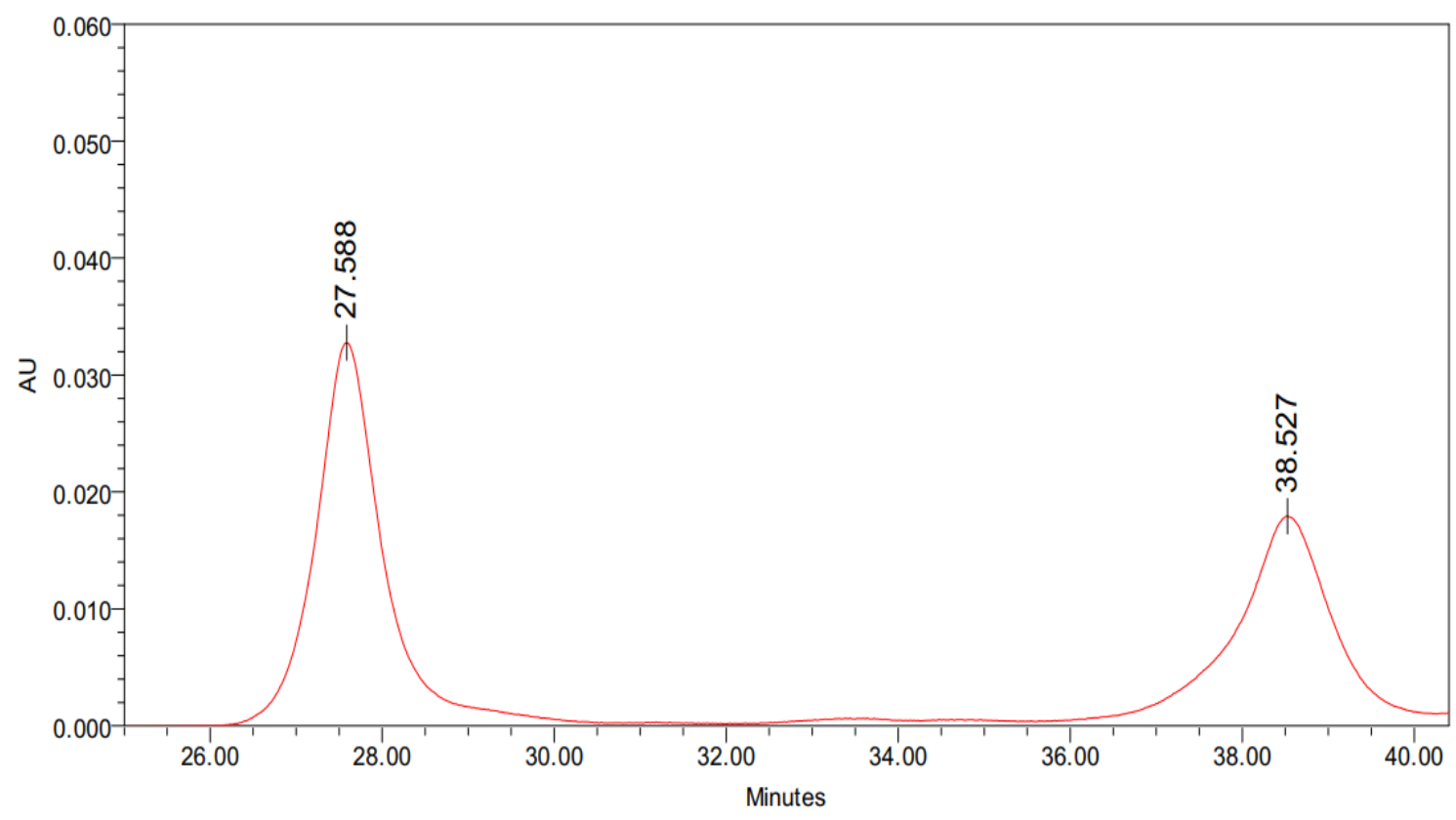

Figure 4.31 Enantioseparation of trans- $\beta$-propylstyrene oxide catalyzed by F103A/F186A-CPO on Whelk-O 1 column at room temperature with pure hexane as mobile phase while the absorbance is monitored at $218.0 \mathbf{~ n m}$. 


\subsection{Conclusion}

The substrate-binding pocket of CPO was proposed to interact with the incoming substrates and restricting their access to the ferryl oxygen of the catalytic heme center. These interactions play an important role in regulating the enantioselectivity of the oxygeninsertion reactions. The bottom of the substrate-binding pocket of CPO was formed by the side chains of Phe103, Val182, and Phe186. These side chains along with those of Leu70 and Ala267 have been shown in docking study to have hydrophobic interaction with dimethylaniline, where CPO can catalyze the oxidative demethylation of the substrate [141].

In the present study, Phe186 was mutated to an alanine residue through site-directed mutagenesis generating the F186A-CPO mutant as well as the double, F103A/F186ACPO, mutant. The characterization of F186A- and F103A/F186A-CPO revealed that these two mutants were quite different than WT-CPO. For instance, the UV-Vis spectra of the two mutants indicated the presence of a low-spin hexa-coordinated species while the spectrum of WT-CPO clearly revealed the heme iron is penta-coordinated. Even though the mutations altered the spin nature, from high-spin to low-spin, of the resting enzyme, the identity of the proximal ligand remained the same. The carbonmonoxy complexes of WT-, F186A-, and F103A/F186A-CPO possess the signature Soret peak at $445 \mathrm{~nm}$ as an indication of a thiolate ligation. The CD spectrum of F186A- and F103A/F186A-CPO was similar to that of WT-CPO as no significant difference was observed for their secondary structure. Further spectroscopic study of these two mutants suggested that these mutations altered the binding affinity of exogenous ligands, such as cyanide, to the heme center of CPO. 
Besides the spectroscopic characterization of the mutants, the influence on the biochemical properties of CPO was also characterized. Interestingly, the single mutation of Phe186 to alanine caused dramatic changes on the catalytic activity of CPO. F186ACPO contains no significant sign of halogenation, chlorination and bromination, activity relative to WT-CPO. Similar results were observed for F103A/F186A-CPO. The change in the halogenating activity observed for F186A- and F103A/F186A-CPO suggested the possible decrease on the redox potential of Compound I, where the oxidation of halide ions is inefficient. This hypothesis is further supported by the abolishment of the catalatic activity of F186A-CPO, where the oxidation of hydrogen peroxide by Compound I is also prohibited. Another plausible reasoning was the mutation influenced the catalytic heme center rendering the reaction with polar substrates, such as chloride ion and hydrogen peroxide, unfavorable. The peroxidase activity of F186A- and F103A/F186A-CPO was increased by more than 8 -fold at $\mathrm{pH} 4.00$ and 5.00 relative to WT-CPO. This can be explained by the inverse relationship between redox potential of the heme center and the pKa of the distal ligand. Therefore, the decrease in the redox potential would regulate the increase in the pKa of F186A- and F103A/F186A-CPO's Compound II, favoring the formation of the protonated species. Besides the peroxidation pathway, the oxygeninsertion pathway was also enhanced for the $\mathrm{CPO}$ mutants. Throughout the tested $\mathrm{pH}$ range 3.00-5.50, F186A- and F103A/F186A-CPO displayed a minimum of 5-fold enhancement relative to WT-CPO. The biochemical properties of F186A- and F103A/F186A-CPO seem to be a better applicable biocatalyst than WT-CPO.

To further investigate the possible application of the CPO mutants, the enantioselectivity of F186A- and F103A/F186A-CPO catalyzed epoxidation reactions of 
styrene, cis- $\beta$-methylstyrene, trans- $\beta$-methylstyrene, trans- $\beta$-ethylstyrene, and trans- $\beta$ propylstyrene were examined with HPLC Whelk-O 1 column. As expected, F186A- and F103A/F186A-CPO displayed different epoxidation behavior relative to WT-CPO. For example, the reaction catalyzed by WT-, F186A-, F103A/F186A-CPO of styrene epoxidation yielded different ee values. As F186A-CPO, the ee value is $3.5 \%$ which decreased by more than $30 \%$ as to the WT-CPO catalyzed styrene epoxidation reaction. The change in the ee values might arise from the mutations on the Phe103/Phe186 clamp, where the presence of a larger substrate-binding pocket disturbed the interactions between styrene and the phenylalanine residues as well as the binding conformation of the substrate during the binding process. This proposition was further supported by the epoxidation reaction of trans- $\beta$-methylstyrene. Trans- $\beta$-methylstyrene is a poor substrate for WT-CPO as the ee value is only $8 \%$. However, F186A- and F103A/F186A-CPO displayed a higher enantioselectivity for trans- $\beta$-methylstyrene as the ee value is $19 \%$ and $55 \%$, respectively. Interestingly, the epoxidation of trans- $\beta$-ethylstyrene catalyzed by the two mutants displayed higher enantioselectivity for this substrate but the conformation preference is different, with the $(S)$-enantiomer as the major product. As for trans- $\beta$-propylstyrene, the enantioselectivity for this reaction catalyzed by WT-, F186A-, and F103A/F186A-CPO has no significant difference.

WT-CPO catalyzes epoxidation of cis- $\beta$-methylstyrene with high enantioselectivity, suggesting the proper fit of the substrate in the substrate-binding pocket. My result confirms the high enantioselectivity of this reaction. As for the CPO mutants, the reaction yielded two products indicating the decrease in the enantioselectivity for cis- $\beta$ methylstyrene. Since the interactions between WT-CPO and the substrate is disturbed by 
the mutation(s), the ee value decreased as the space in the substrate-binding pocket increased. The enantioselective profile of F103A-, F186A-, and F103A/F186A-CPO suggests Phe186 plays a role in modulating the enantioselectivity of as illustrate in Table 4.5 .

Table 4.5 Enantioselectivity (ee\%) comparison between WT-, F103A-, F186A-, and F103A/F186A-CPO.

\begin{tabular}{|l|l|l|l|l|}
\hline Proteins & Styrene & $\begin{array}{l}\text { Cis- } \beta- \\
\text { methylstyrene }\end{array}$ & $\begin{array}{l}\text { Trans- } \beta- \\
\text { methylstyrene }\end{array}$ & $\begin{array}{l}\text { Trans- } \beta- \\
\text { ethylstyrene }\end{array}$ \\
\hline WT-CPO & $44 \%$ & $\sim 99 \%$ & $8 \%$ & $3 \%$ \\
\hline F103A-CPO [124] & $2 \%$ & $5 \%$ & $50 \%$ & $34 \%$ \\
\hline F186A-CPO & $3 \%$ & $42 \%$ & $19 \%$ & $23 \%(S)$ \\
\hline F103A/F186A-CPO & $9 \%$ & $12 \%$ & $55 \%$ & $21 \%(S)$ \\
\hline
\end{tabular}

Mutation on Phe186 favors the yield of the $(S)$-enantiomer on the epoxidation of trans$\beta$-ethylstyrene but this preference was no observed for F103A-CPO. Phe103 plays a more significant role relative to Phe186 as F103A-CPO displayed a higher enantioselectivity for trans-substituent styrene derivative reactions. For example, F103A-CPO catalyzes the epoxidation of trans- $\beta$-methylstyrene and trans- $\beta$-ethylstyrene with the ee values of $51 \%$ and 48\%, respectively [124]. The different degree of influence between Phe103 and Phe186 might possibly be due to the flexibility of Phe103, as the crystal structure indicates the displacement of Phe103 during the binding of CPD, while Phe186 remained intact [70]. Furthermore, a computational study on the distal pocket of CPO's influence on the epoxidation of $c i s-\beta$-methylstyrene provided us an insight on the flexibility of Phe103 and Phe186 that during the breathing of the distal pocket, Phe103 is displaced more significantly than Phe186 [142]. 
My work further the understanding on the influence of the distal pocket of CPO toward the catalytic properties as well as the enantioselective profile of the enzyme. Furthermore, these results added on the possible industrial applications of CPO, as well as other hemethiolate enzymes and through protein engineering, certain aspect of the structure can be modified to favor certain reactions such as enantioselective peroxygenation reactions. 


\section{REFERENCES}

1. Zhang, L., Heme Biology: The Secret Life of Heme in Regulating Diverse Biological Processes. World Scientific, 2011.

2. $\quad$ Poulos, T.L., Heme Enzyme Structure and Function. Chem Rev, 2014. 114(7): p. 3919-62.

3. Ortiz de Montellano, P.R., Control of the catalytic activity of prosthetic heme by the structure of hemoproteins. Accounts of Chemical Research, 1987. 20(8): p. 289294.

4. Poulos, T.L., Heme Enzyme Structure and Function. Chemical Reviews, 2014. 114(7): p. 3919-3962.

5. Dawson, J.H. and M. Sono, Cytochrome P-450 and chloroperoxidase: thiolateligated heme enzymes. Spectroscopic determination of their active-site structures and mechanistic implications of thiolate ligation. Chemical Reviews, 1987. 87(5): p. 1255-1276.

6. Denisov, I.G., et al., Structure and chemistry of cytochrome P450. Chemical reviews, 2005. 105(6): p. 2253-2278.

7. Weiner, L.M., Magnetic Resonance Study of the Structure and Functions of Cytochrome P450. CRC critical reviews in biochemistry, 1986. 20(2): p. 139-200.

8. Poulos, T.L., The role of the proximal ligand in heme enzymes. Journal of Biological Inorganic Chemistry, 1996. 1(4): p. 356-359.

9. Yoshioka, S., et al., Proximal cysteine residue is essential for the enzymatic activities of cytochrome P450cam. European Journal of Biochemistry, 2001. 268(2): p. 252-9.

10. Yoshioka, S., et al., Roles of the axial push effect in cytochrome P450cam studied with the site-directed mutagenesis at the heme proximal site. Journal of Inorganic Biochemistry, 2000. 81(3): p. 141-51.

11. Yoshioka, S., et al., Roles of the proximal hydrogen bonding network in cytochrome P450cam-catalyzed oxygenation. Journal of the American Chemical Society, 2002. 124(49): p. 14571-14579.

12. Poulos, T.L. and R. Raag, Cytochrome P450cam: crystallography, oxygen activation, and electron transfer. The FASEB Journal, 1992. 6(2): p. 674-9.

13. Sono, M., et al., Heme-Containing Oxygenases. Chemical Reviews, 1996. 96(7): p. 2841-2888. 
14. Alberta, J.A., L.A. Andersson, and J.H. Dawson, Spectroscopic characterization of secondary amine mono-oxygenase. Comparison to cytochrome P-450 and myoglobin. J Biol Chem, 1989. 264(34): p. 20467-73.

15. Munro, A.W., H.M. Girvan, and K.J. McLean, Variations on a (T)heme - Novel Mechanisms, Redox Partners and Catalytic Functions in the Cytochrome P450 Superfamily. Nat Prod Rep, 2007. 24(3): p. 585-609.

16. Poulos, T.L., Structural biology of heme monooxygenases. Biochem Biophys Res Commun, 2005. 338(1): p. 337-45.

17. Munro, A.W., H.M. Girvan, and K.J. McLean, Variations on a (t)heme--novel mechanisms, redox partners and catalytic functions in the cytochrome P450 superfamily. Nat Prod Rep, 2007. 24(3): p. 585-609.

18. Gajhede, M., et al., Crystal structure of horseradish peroxidase $C$ at 2.15 A resolution. Nat Struct Biol, 1997. 4(12): p. 1032-8.

19. Guo, M., et al., Crystal structure and characterization of a cytochrome c peroxidase-cytochrome c site-specific cross-link. Proceedings of the National Academy of Sciences, 2004. 101(16): p. 5940-5945.

20. Schoemaker, H.E. and K. Piontek, On the interaction of lignin peroxidase with lignin. Pure and applied chemistry, 1996. 68(11): p. 2089-2096.

21. Zamocky, M., et al., Independent evolution of four heme peroxidase superfamilies. Arch Biochem Biophys, 2015. 574: p. 108-19.

22. Torres, E. and M. Ayala, Biocatalysis based on heme peroxidases: peroxidases as potential industrial biocatalysts. 2010: Springer Science \& Business Media.

23. Deisseroth, A. and A.L. Dounce, Catalase: Physical and chemical properties, mechanism of catalysis, and physiological role. Physiol Rev, 1970. 50(3): p. 31975 .

24. Poulos, T.L., et al., The crystal structure of cytochrome c peroxidase. J Biol Chem, 1980. 255(2): p. 575-80.

25. Zámocký, M., P.G. Furtmüller, and C. Obinger, Evolution of structure and function of Class I peroxidases. Archives of Biochemistry and Biophysics, 2010. 500(1): p. 45-57.

26. Stern, J.O. and J. Peisach, A model compound study of the CO-adduct of cytochrome P-450. J Biol Chem, 1974. 249(23): p. 7495-8. 
27. Omura, T. and R. Sato, The Carbon Monoxide-binding Pigment of Liver Microsomes: I. EVIDENCE FOR ITS HEMOPROTEIN NATURE. J Biol Chem, 1964. 239: p. 2370-8.

28. Hrycay, E.G. and S.M. Bandiera, Monooxygenase, peroxidase and peroxygenase properties and reaction mechanisms of cytochrome P450 enzymes. Adv Exp Med Biol, 2015. 851: p. 1-61.

29. Auclair, K., P. Moënne-Loccoz, and P.R. Ortiz de Montellano, Roles of the Proximal Heme Thiolate Ligand in Cytochrome P450cam. Journal of the American Chemical Society, 2001. 123(21): p. 4877-4885.

30. Littlechild, J., Haloperoxidases and their role in biotransformation reactions. Current opinion in chemical biology, 1999. 3(1): p. 28-34.

31. Hofrichter, M. and R. Ullrich, Heme-thiolate haloperoxidases: versatile biocatalysts with biotechnological and environmental significance. Appl Microbiol Biotechnol, 2006. 71(3): p. 276-88.

32. Rousseau, D.L., et al., Ligand-protein interactions in nitric oxide synthase. Journal of Inorganic Biochemistry, 2005. 99(1): p. 306-23.

33. Morris, D.R. and L.P. Hager, Chloroperoxidase. I. Isolation and properties of the crystalline glycoprotein. The Journal of Biological Chemistry, 1966. 241(8): p. $1763-8$.

34. Hager, L.P., et al., Chloroperoxidase. II. Utilization of halogen anions. The Journal of Biological Chemistry, 1966. 241(8): p. 1769-77.

35. Brown, F.S. and L.P. Hager, Chloroperoxidase. IV. Evidence for an Ionic Electrophilic Substitution Mechanism. Journal of the American Chemical Society, 1967. 89(3): p. 719-720.

36. Thomas, J.A., D.R. Morris, and L.P. Hager, Chloroperoxidase: VII. Classica Peroxidatic, Catalatic, and Halogenating forms of the Enzyme Journal of Biological Chemistry, 1970. 245(12): p. 3129-3134.

37. Hollenberg, P.F. and L.P. Hager, The P-450 nature of the carbon monoxide complex of ferrous chloroperoxidase. J Biol Chem, 1973. 248(7): p. 2630-3.

38. Sundaramoorthy, M., J. Terner, and T.L. Poulos, The crystal structure of chloroperoxidase: a heme peroxidase--cytochrome P450 functional hybrid. Structure, 1995. 3(12): p. 1367-77.

39. Palcic, M.M., et al., Spectrum of chloroperoxidase compound I. Biochemical and Biophysical Research Communications, 1980. 94(4): p. 1123-1127. 
40. Rutter, R., et al., Chloroperoxidase compound I: electron paramagnetic resonance and Mössbauer studies. Biochemistry, 1984. 23(26): p. 6809-6816.

41. Green, M.T., J.H. Dawson, and H.B. Gray, Oxoiron (IV) in chloroperoxidase compound II is basic: Implications for P450 chemistry. Science, 2004. 304(5677): p. 1653-1656.

42. Stone, K.L., R.K. Behan, and M.T. Green, X-ray absorption spectroscopy of chloroperoxidase compound I: insight into the reactive intermediate of $\mathrm{P} 450$ chemistry. Proceedings of the National Academy of Sciences of the United States of America, 2005. 102(46): p. 16563-16565.

43. Egawa, T., et al., Observation of the FeIV=O stretching Raman band for a thiolateligated heme protein Compound I of chloroperoxidase. FEBS letters, 1992. 305(3): p. 206-208.

44. Rittle, J. and M.T. Green, Cytochrome P450 Compound I: Capture, Characterization, and C-H Bond Activation Kinetics. Science, 2010. 330(6006): p. 933-937.

45. Ullrich, R. and M. Hofrichter, The haloperoxidase of the agaric fungus Agrocybe aegerita hydroxylates toluene and naphthalene. FEBS Lett, 2005. 579(27): p. 624750.

46. Ullrich, R. and M. Hofrichter, Enzymatic hydroxylation of aromatic compounds. Cell Mol Life Sci, 2007. 64(3): p. 271-93.

47. Ullrich, R., et al., Novel haloperoxidase from the agaric basidiomycete Agrocybe aegerita oxidizes aryl alcohols and aldehydes. Applied and environmental microbiology, 2004. 70(8): p. 4575-4581.

48. Hofrichter, M., et al. Fungal unspecific peroxygenases: a new generation of oxygen-transferring biocatalysts. in Proceedings of the 8th international conference on Mushroom Biology and Mushroom Products (ICMBMP8). 2014.

49. Lundell, T., et al., New Mechanism of the $C \alpha-C \beta$ Cleavage in Non-Phenolic Arylglycerol $\beta$-Aryl Ether Lignin Substructures Catalyzed by Lignin Peroxidase. Holzforschung-International Journal of the Biology, Chemistry, Physics and Technology of Wood, 1993. 47(3): p. 219-224.

50. Ullrich, R., et al., Novel haloperoxidase from the agaric basidiomycete Agrocybe aegerita oxidizes aryl alcohols and aldehydes. Appl Environ Microbiol, 2004. 70(8): p. 4575-81.

51. Pecyna, M.J., et al., Molecular characterization of aromatic peroxygenase from Agrocybe aegerita. Appl Microbiol Biotechnol, 2009. 84(5): p. 885-97. 
52. Peter, S., et al., Selective hydroxylation of alkanes by an extracellular fungal peroxygenase. Febs j, 2011. 278(19): p. 3667-75.

53. Hofrichter, M., et al., Fungal unspecific peroxygenases: heme-thiolate proteins that combine peroxidase and cytochrome p450 properties. Adv Exp Med Biol, 2015. 851: p. 341-68.

54. Poraj-Kobielska, M., et al., Preparation of human drug metabolites using fungal peroxygenases. Biochem Pharmacol, 2011. 82(7): p. 789-96.

55. Shaw, P.D. and L.P. Hager, Biological chlorination vi. chloroperoxidase: a component of the $\beta$-ketoadipate chlorinase system. Journal of Biological Chemistry, 1961. 236(6): p. 1626-1630.

56. Shaw, P.D. and L.P. Hager, Biological chlorination. III. beta-Ketoadipate chlorinase: a soluble enzyme system. The Journal of Biological Chemistry, 1959. 234: p. 2565-9.

57. Ortiz-Bermudez, P., E. Srebotnik, and K.E. Hammel, Chlorination and cleavage of lignin structures by fungal chloroperoxidases. Appl Environ Microbiol, 2003. 69(8): p. 5015-8.

58. Manoj, K.M. and L.P. Hager, Chloroperoxidase, a janus enzyme. Biochemistry, 2008. 47(9): p. 2997-3003.

59. Morris, D.R. and L.P. Hager, Chloroperoxidase I. Isolation and properties of the crystalline glycoprotein. Journal of Biological Chemistry, 1966. 241(8): p. 17631768 .

60. Fang, G.H., et al., Cloning and sequencing of chloroperoxidase cDNA. Nucleic Acids Research, 1986. 14(20): p. 8061-8071.

61. Poulos, T.L., B.C. Finzel, and A.J. Howard, High-resolution crystal structure of cytochrome P450cam. J Mol Biol, 1987. 195(3): p. 687-700.

62. Murthy, M.R.N., et al., Structure of beef liver catalase. Journal of Molecular Biology, 1981. 152(2): p. 465-499.

63. Ueyama, N., et al., Cytochrome P-450 Model (Porphinato)(thiolato)iron(III) Complexes with Single and Double NH..S Hydrogen Bonds at the Thiolate Site. Journal of the American Chemical Society, 1996. 118(50): p. 12826-12827.

64. Galinato, M.G.I., et al., Elucidating the Role of the Proximal Cysteine HydrogenBonding Network in Ferric Cytochrome P450cam and Corresponding Mutants Using Magnetic Circular Dichroism Spectroscopy. Biochemistry, 2011. 50(6): p. 1053-1069. 
65. Ogliaro, F., et al., Medium Polarization and Hydrogen Bonding Effects on Compound I of Cytochrome P450: What Kind of a Radical Is It Really? Journal of the American Chemical Society, 2000. 122(51): p. 12892-12893.

66. Suzuki, N., et al., Novel Iron Porphyrin-Alkanethiolate Complex with

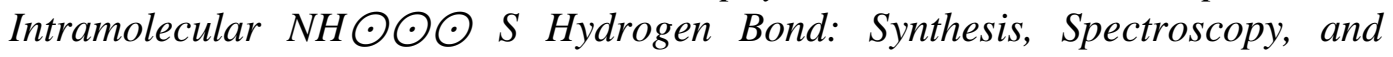
Reactivity. Journal of the American Chemical Society, 1999. 121(49): p. 1157111572.

67. Pardillo, A.D., A.N. Morozov, and D.C. Chatfield, Proximal Pocket Hydrogen Bonds Significantly Influence the Mechanism of Chloroperoxidase Compound I Formation. The Journal of Physical Chemistry B, 2015. 119(39): p. 12590-12602.

68. Ueno, T., et al., Role of $\alpha$-Helix Conformation Cooperating with NH $\cdots$ S Hydrogen Bond in the Active Site of Cytochrome P-450 and Chloroperoxidase: Synthesis and Properties of [MIII(OEP)(Cys-Helical Peptide $)](M=F e$ and $G a)$. Journal of the American Chemical Society, 1998. 120(47): p. 12264-12273.

69. Ueyama, N., et al., Synthesis and Properties of Octaethylporphinato (arenethiolato) iron (III) Complexes with Intramolecular NH $\odot \odot S$ Hydrogen Bond: Chemical Function of the Hydrogen Bond. Inorganic Chemistry, 1998. 37(10): p. 2415-2421.

70. Kuhnel, K., et al., Crystal structures of chloroperoxidase with its bound substrates and complexed with formate, acetate, and nitrate. The Journal of Biological Chemistry, 2006. 281(33): p. 23990-8.

71. Makino, R., R. Chiang, and L.P. Hager, Oxidation-reduction potential measurements on chloroperoxidase and its complexes. Biochemistry, 1976. 15(21): p. $4748-4754$.

72. Everse, J., M.B. Grisham, and K.E. Everse, Peroxidases in chemistry and biology. Vol. 1. 1990: CRC press.

73. Sundaramoorthy, M., J. Terner, and T.L. Poulos, Stereochemistry of the chloroperoxidase active site: crystallographic and molecular-modeling studies. Chem Biol, 1998. 5(9): p. 461-73.

74. Filizola, M. and G.H. Loew, Probing the role of protein environment in compound Iformation of chloroperoxidase (CPO). Journal of the American Chemical Society, 2000. 122(15): p. 3599-3605.

75. Chen, H., et al., Quantum Mechanical/Molecular Mechanical Study on the Mechanisms of Compound I Formation in the Catalytic Cycle of Chloroperoxidase: An Overview on Heme Enzymes. The Journal of Physical Chemistry B, 2008. 112(31): p. 9490-9500. 
76. Kühnel, K., et al., Structure and quantum chemical characterization of chloroperoxidase compound 0 , a common reaction intermediate of diverse heme enzymes. Proceedings of the National Academy of Sciences, 2007. 104(1): p. 99104.

77. Sundaramoorthy, M., J. Terner, and T.L. Poulos, Stereochemistry of the chloroperoxidase active site: crystallographic and molecular-modeling studies. Chemistry \& biology, 1998. 5(9): p. 461-473.

78. Green, M.T., J.H. Dawson, and H.B. Gray, Oxoiron(IV) in chloroperoxidase compound II is basic: implications for P450 chemistry. Science, 2004. 304(5677): p. 1653-6.

79. Chiang, R., et al., Compound X. An intermediate in enzymatic halogenation. Journal of Biological Chemistry, 1976. 251(20): p. 6340-6346.

80. Woggon, W.-D., H.-A. Wagenknecht, and C. Claude, Synthetic active site analogues of heme-thiolate proteins: Characterization and identification of intermediates of the catalytic cycles of cytochrome P450cam and chloroperoxidase. Journal of Inorganic Biochemistry, 2001. 83(4): p. 289-300.

81. Hofrichter, M., et al., New and classic families of secreted fungal heme peroxidases. Applied Microbiology and Biotechnology, 2010. 87(3): p. 871-897.

82. van Rantwijk, F. and R.A. Sheldon, Selective oxygen transfer catalysed by heme peroxidases: synthetic and mechanistic aspects. Current Opinion in Biotechnology, 2000. 11(6): p. 554-564.

83. Sun, W., et al., Catalase activity of chloroperoxidase and its interaction with peroxidase activity. Biochemistry and Cell Biology, 1994. 72(7-8): p. 321-331.

84. Alfonso-Prieto, M., et al., The Molecular Mechanism of the Catalase Reaction. Journal of the American Chemical Society, 2009. 131(33): p. 11751-11761.

85. Claude, C., Enzyme models of chloroperoxidase and catalase. 2001, University_of_Basel.

86. Libby, R.D., et al., Chloroperoxidase halogenation reactions. Chemical versus enzymic halogenating intermediates. J Biol Chem, 1982. 257(9): p. 5030-7.

87. Geigert, J., et al., Haloperoxidases: enzymatic synthesis of $\alpha$, $\beta$-halohydrins from gaseous alkenes. Applied and environmental microbiology, 1983. 45(2): p. 366374.

88. Geigert, J., et al., Novel haloperoxidase reaction: synthesis of dihalogenated products. Applied and environmental microbiology, 1983. 45(5): p. 1575-1581. 
89. Geigert, J., S.L. Neidleman, and D.J. Dalietos, Novel haloperoxidase substrates. Alkynes and cyclopropanes. Journal of Biological Chemistry, 1983. 258(4): p. 2273-2277.

90. Pickard, M.A., T.A. Kadima, and R.D. Carmichael, Chloroperoxidase, a peroxidase with potential. Journal of industrial microbiology, 1991. 7(4): p. 235241.

91. Hallenberg, P.F. and L.P. Hager, Purification of chloroperoxidase from Caldariomyces fumago. Methods in enzymology, 1978. 52: p. 521-9.

92. Wannstedt, C., D. Rotella, and J. Siuda, Chloroperoxidase mediated halogenation of phenols. Bulletin of environmental contamination and toxicology, 1990. 44(2): p. 282-287.

93. Vazquez-Duhalt, R., M. Ayala, and F.J. Marquez-Rocha, Biocatalytic chlorination of aromatic hydrocarbons by chloroperoxidase of Caldariomyces fumago. Phytochemistry, 2001. 58(6): p. 929-33.

94. Yaipakdee, P. and L.W. Robertson, Enzymatic halogenation of flavanones and flavones. Phytochemistry, 2001. 57(3): p. 341-347.

95. Niedan, V., I. Pavasars, and G. Oberg, Chloroperoxidase-mediated chlorination of aromatic groups in fulvic acid. Chemosphere, 2000. 41(5): p. 779-85.

96. Osborne, R.L., et al., Caldariomyces fumago Chloroperoxidase Catalyzes the Oxidative Dehalogenation of Chlorophenols by a Mechanism Involving Two OneElectron Steps. Journal of the American Chemical Society, 2007. 129(48): p. 14838-14839.

97. Osborne, R.L., et al., C. fumago chloroperoxidase is also a dehaloperoxidase: oxidative dehalogenation of halophenols. Journal of the American Chemical Society, 2006. 128(4): p. 1036-7.

98. La Rotta H, C.E. and E. PS Bon, 4-chlorophenol degradation by chloroperoxidase from Caldariomyces fumago. Applied biochemistry and biotechnology, 2002. 98(1): p. 191-203.

99. Murphy, C.D., Fluorophenol oxidation by a fungal chloroperoxidase. Biotechnology Letters, 2007. 29(1): p. 45-49.

100. Longoria, A., R. Tinoco, and R. Vazquez-Duhalt, Chloroperoxidase-mediated transformation of highly halogenated monoaromatic compounds. Chemosphere, 2008. 72(3): p. 485-90. 
101. Díaz-Díaz, G., et al., Kinetic study of the oxidative dehalogenation of 2,4,6trichlorophenol catalyzed by chloroperoxidase. Journal of Molecular Catalysis B: Enzymatic, 2010. 66(3): p. 332-336.

102. Colonna, S., et al., Recent biotechnological developments in the use of peroxidases. Trends in Biotechnology, 1999. 17(4): p. 163-168.

103. Vargas, R.R., et al., Asymmetric sulfoxidation of a $\beta$-carbonyl sulfide series by chloroperoxidase. Tetrahedron: Asymmetry, 1999. 10(16): p. 3219-3227.

104. Van Deurzen, M., et al., Chloroperoxidase catalyzed oxidations in t-butyl alcohol/water mixtures. Journal of Molecular Catalysis A: Chemical, 1997. 117(13): p. 329-337.

105. Ayala, M., et al., Substrate Specificity and Ionization Potential in Chloroperoxidase-Catalyzed Oxidation of Diesel Fuel. Environmental Science \& Technology, 2000. 34(13): p. 2804-2809.

106. Carreno, M.C., Applications of Sulfoxides to Asymmetric Synthesis of Biologically Active Compounds. Chemical Reviews, 1995. 95(6): p. 1717-1760.

107. Gao, F., et al., Enzymatic synthesis of $(R)$-modafinil by chloroperoxidase-catalyzed enantioselective sulfoxidation of 2-(diphenylmethylthio) acetamide. Biochemical Engineering Journal, 2015. 93: p. 243-249.

108. Zaks, A. and D.R. Dodds, Chloroperoxidase-catalyzed asymmetric oxidations: substrate specificity and mechanistic study. Journal of the American Chemical Society, 1995. 117(42): p. 10419-10424.

109. Allain, E.J., et al., Highly enantioselective epoxidation of disubstituted alkenes with hydrogen peroxide catalyzed by chloroperoxidase. Journal of the American Chemical Society, 1993. 115(10): p. 4415-4416.

110. Dexter, A.F., et al., Highly Enantioselective Epoxidation of 1,1-Disubstituted Alkenes Catalyzed by Chloroperoxidase. Journal of the American Chemical Society, 1995. 117(23): p. 6412-6413.

111. Lakner, F.J. and L.P. Hager, Chloroperoxidase as enantioselective epoxidation catalyst: an efficient synthesis of $(R)-(-)$-mevalonolactone. The Journal of organic chemistry, 1996. 61(11): p. 3923-3925.

112. Lakner, F.J. and L.P. Hager, Chloroperoxidase-mediated asymmetric epoxidation. Synthesis of $(R)$-dimethyl 2-methylaziridine-1,2-dicarboxylate-a potential $\alpha$ methylamino acid synthon. Tetrahedron: Asymmetry, 1997. 8(21): p. 3547-3550. 
113. Manoj, K.M. and L.P. Hager, Utilization of peroxide and its relevance in oxygen insertion reactions catalyzed by chloroperoxidase. Biochimica et Biophysica Acta (BBA) - Protein Structure and Molecular Enzymology, 2001. 1547(2): p. 408-417.

114. Manoj, K.M., et al., A Kinetic Epoxidation Assay for Chloroperoxidase. Biochemical and Biophysical Research Communications, 1999. 266(2): p. 301303.

115. $\mathrm{Hu}, \mathrm{S}$. and L.P. Hager, Highly Enantioselective Propargylic Hydroxylations Catalyzed by Chloroperoxidase. Journal of the American Chemical Society, 1999. 121(4): p. 872-873.

116. Sanfilippo, C. and G. Nicolosi, Catalytic behaviour of chloroperoxidase from Caldariomyces fumago in the oxidation of cyclic conjugated dienes. Tetrahedron: Asymmetry, 2002. 13(17): p. 1889-1892.

117. Sanfilippo, C., A. Patti, and G. Nicolosi, Asymmetric oxidation of 1,3cyclohexadiene catalysed by chloroperoxidase from Caldariomyces fumago. Tetrahedron: Asymmetry, 2000. 11(16): p. 3269-3272.

118. Miller, V.P., R.A. Tschirret-Guth, and P.R. Ortiz de Montellano, Chloroperoxidase-catalyzed benzylic hydroxylation. Arch Biochem Biophys, 1995. 319(2): p. 333-40.

119. Aguila, S., et al., Stereoselective oxidation of $R-(+)$-limonene by chloroperoxidase from Caldariomyces fumago. Green Chemistry, 2008. 10(6): p. 647-653.

120. Kiljunen, E. and L.T. Kanerva, Novel applications of chloroperoxidase: enantioselective oxidation of racemic epoxyalcohols. Tetrahedron: Asymmetry, 1999. 10(18): p. 3529-3535.

121. $\mathrm{Hu}, \mathrm{S}$. and J.S. Dordick, Highly enantioselective oxidation of ciscyclopropylmethanols to corresponding aldehydes catalyzed by chloroperoxidase. J Org Chem, 2002. 67(1): p. 314-7.

122. Masdeu, G., et al., Chloroperoxidase-catalyzed amino alcohol oxidation: Substrate specificity and novel strategy for the synthesis of $\mathrm{N}$-Cbz-3-aminopropanal. Process Biochemistry, 2016. 51(9): p. 1204-1211.

123. Jiang, L., Mechanisms of chloroperoxidase-catalyzed enantioselective reactions as probed by site-directed mutagenesis and isotopic labeling. ProQuest ETD Collection for FIU. , 2012.

124. Wang, Z., Characterization of recombinant chloroperoxidase, and F103A and C29H/C79H/C87H mutants". 2011, Florida International University: ProQuest ETD Collection for FIU. Paper AAI3472067. . 
125. Yi, X., et al., Examining the role of glutamic acid 183 in chloroperoxidase catalysis. The Journal of Biological Chemistry, 2003. 278(16): p. 13855-9.

126. Shersher, E., The Influence of the Proximal Thiolate Ligand and Hydrogen Bond Network of the Proximal Helix on the Structural and Biochemical Properties of Chloroperoxidase. FIU Electronic Theses and Dissertations, 2016. 2483.

127. Yi, X., et al., Replacement of the proximal heme thiolate ligand in chloroperoxidase with a histidine residue. Proc Natl Acad Sci U S A, 1999. 96(22): p. 12412-7.

128. Giovannetti, R., The use of spectrophotometry UV-Vis for the study of porphyrins, in Macro to nano spectroscopy. 2012, InTech.

129. Greenfield, N.J., Using circular dichroism spectra to estimate protein secondary structure. Nature protocols, 2006. 1(6): p. 2876.

130. Chenprakhon, P., et al., Measuring Binding Affinity of Protein-Ligand Interaction Using Spectrophotometry: Binding of Neutral Red to Riboflavin-Binding Protein. Journal of chemical education, 2010. 87(8): p. 829-831.

131. Poulos, T.L. and J. Kraut, The stereochemistry of peroxidase catalysis. Journal of Biological Chemistry, 1980. 255(17): p. 8199-8205.

132. Gumiero, A., et al., Nature of the ferryl heme in compounds I and II. Journal of Biological Chemistry, 2011. 286(2): p. 1260-1268.

133. Casadei, C.M., et al., Neutron cryo-crystallography captures the protonation state offerryl heme in a peroxidase. Science, 2014. 345(6193): p. 193-197.

134. Horvath, C.G., B. Preiss, and S.R. Lipsky, Fast liquid chromatography. Investigation of operating parameters and the separation of nucleotides on pellicular ion exchangers. Analytical chemistry, 1967. 39(12): p. 1422-1428.

135. Zhao, C.F., S. Diemert, and N.M. Cann, Rational optimization of the Whelk-O1 chiral stationary phase using molecular dynamics simulations. J Chromatogr A, 2009. 1216(32): p. 5968-78.

136. Del Rio, A., et al., Theoretical reassessment of Whelk-O1 as an enantioselective receptor for 1-(4-halogeno-phenyl)-1-ethylamine derivatives. Chirality, 2004. 16 Suppl: p. S1-11.

137. Pirkle, W.H. and C.J. Welch, Chromatographic and $1 H$ NMR support for a proposed chiral recognition model. Journal of Chromatography A, 1994. 683(2): p. 347-353.

138. Campbell, B.N., Jr., et al., A kinetic study of the binding of carbon monoxide to ferrous chloroperoxidase. Biochemistry, 1982. 21(18): p. 4343-9. 
139. Magora, A., S. Abu-Lafi, and S. Levin, Comparison of the enantioseparation of racemic uridine analogs on Whelk-O 1 and ChiralPak-AD columns. J Chromatogr A, 2000. 866(2): p. 183-94.

140. Chen, T., Chloroperoxidase Catalyzed Enantioselective Epoxidation of Selected Olefins and Regiospecific Degradation of Dimethylsulfoniopropionate. 2011, Florida International University: FIU Electronic Theses and Dissertations. Paper 514.

141. Kedderis, G.L. and P. Hollenberg, Steady state kinetics of chloroperoxidasecatalyzed N-demethylation reactions. Journal of Biological Chemistry, 1983. 258(20): p. 12413-12419.

142. Morozov, A.N. and D.C. Chatfield, Chloroperoxidase-Catalyzed Epoxidation of cis- $\beta$-Methylstyrene: Distal Pocket Flexibility Tunes Catalytic Reactivity. The Journal of Physical Chemistry B, 2012. 116(43): p. 12905-12914. 


\section{APPENDIX}

Figure A1. DNA sequence of full-length WT CPO gene.

ATGTTCTCCAAGGTCCTTCCCTTCGTGGGAGCGGTTGCCGCCCTCCCTCACTC CGTCCGTCAGGAGCCTGGCTCCGGCATTGGCTACCCATACGACAACAACACC CTGCCATATGTCGCCCCAGGTCCTACCGACTCTCGTGCTCCTTGCCCAGCTCT GAACGCTCTTGCCAACCACGGTTACATTCCTCACGATGGCCGTGCCATCAGC AGGGAGACCCTCCAGAACGCTTTCCTCAACCACATGGGTATTGCCAACTCCG TCATTGAGCTTGCTCTGACCAACGCCTTCGTCGTCTGCGAGTACGTTACTGGC TCCGACTGTGGTGACAGCCTTGTCAACCTGACTCTGCTCGCCGAGCCCCACGC TTTCGAGCACGACCACTCCTTCTCCCGCAAGGATTACAAGCAGGGTGTCGCC AACTCCAACGACTTCATCGACAACAGGAACTTCGATGCCGAGACCTTCCAGA CCTCTCTGGATGTCGTTGCAGGCAAGACCCACTTCGACTATGCCGACATGAA CGAGATCCGCCTTCAGCGCGAGTCCCTCTCCAACGAGCTTGACTTCCCCGGTT GGTTCACCGAGTCCAAGCCAATCCAGAACGTCGAGTCTGGCTTCATCTTCGCC CTTGTCTCTGACTTCAACCTGCCCGACAACGATGAGAACCCTCTGGTTCGCAT TGACTGGTGGAAGTACTGGTTCACCAACGAGTCCTTCCCATACCACCTCGGCT GGCACCCCCCGTCTCCAGCCAGGGAGATCGAGTTCGTCACCTCCGCCTCCTCC GCTGTCCTGGCTGCCTCTGTCACCTCTACTCCATCTTCCCTTCCATCCGGTGCC ATCGGCCCAGGTGCCGAGGCTGTCCCTCTCTCCTTCGCCTCCACCATGACCCC ATTCCTCCTCGCCACCAATGCTCCTTACTACGCCCAGGACCCAACTCTCGGCC CCAACGACAAGCGTGAGGCTGCCCCAGCTGCCACCACCTCCATGGCCGTCTT CAAGAACCCATACCTCGAGGCCATTGGCACCCAGGACATCAAGAACCAGCA GGCTTACGTCAGCTCCAAGGCTGCTGCCATGGCCTCTGCCATGGCCGCCAAC AAGGCCCGCAACCTTTAA

Figure A2. Amino acid sequence of WT-CPO.

XEPGSGIGYPYDNNTLPYVAPGPTDSRAPCPALNALANHGYIPHDGRAISRETLQ NAFLNHMGIANSVIELALTNAFVVCEYVTGSDCGDSLVNLTLLAEPHAFEHDHSF SRKDYKQGVANSNDFIDNRNFDAETFQTSLDVVAGKTHFDYADMNEIRLQRESL SNELDFPGWFTESKPIQNVESGFIFALVSDFNLPDNDENPLVRIDWWKYWFTNES FPYHLGWHPPSPAREIEFVTSASSAVLAASVTSTPSSLPSGAIGPGAEAVPLSFAST MTPFLLATNAPYYAQDPTLGPND 
VITA

\section{ELWOOD KWONGLAM}

\section{EDUCATION AND EXPERIENCE:}

2013-2018

Ph.D., Biochemistry

Florida International University

Miami, Florida, USA

Concentration: Biochemistry, Heme-thiolate Proteins

Dissertation: Investigating the Role of the Proximal Cysteine

Hydrogen Bonding Network and Distal Pocket in

Chloroperoxidase

2013-2018

Head Teaching Assistant (General Chemistry I and II Lab)

Florida International University

Miami, Florida, USA

2009-2012

Bachelor of Science in Chemistry

Florida International University

Miami, Florida, USA

\section{PUBLICATIONS AND PRESENTATIONS}

Kwong Lam, Elwood; Wang, Xiaotang. "Investigating the effects of the proximal loop on chloroperoxidase's catalysis" (Manuscripts in Preparation)

Kwong Lam, Elwood; Wang, Xiaotang. "Effects of the pro-cys-pro tripeptide stretch on the structural and biochemical properties of chloroperoxidase," 255th ACS National Meeting \& Exposition, New Orleans, LA, United States, March 18-22, 2018 (2018), BIOL195.

Kwong Lam, Elwood; Wang, Xiaotang. "Elucidating the role of the proximal ligand loop in chloroperoxidase catalysis." 254th ACS National Meeting \& Exposition, Washington, DC, United States, August 20-24, 2017 (2017), BIOL-85.

Kwong Lam, Elwood; Wang, Xiaotang. "Investigating the role of the proximal cysteine hydrogen bonding network in chloroperoxidase," Graduate Student Appreciation Week in Florida International University, Miami, FL, United States, March 27-28, 2017 (oral presentation).

Kwong Lam, Elwood; Wang, Xiaotang. "The Role of the Proximal Ligand Loop in Chloroperoxidase Catalysis," Prospect Student Visitation Day in Florida International University, Miami, FL, United States, March 24, 2017 (poster presentation). 